
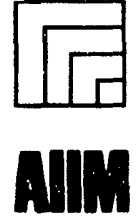

Association for Information and Image Management

1100 Wayne Avenue, Suite 1100

Silver Spring. Maryland 20910

$301 / 587-8202$

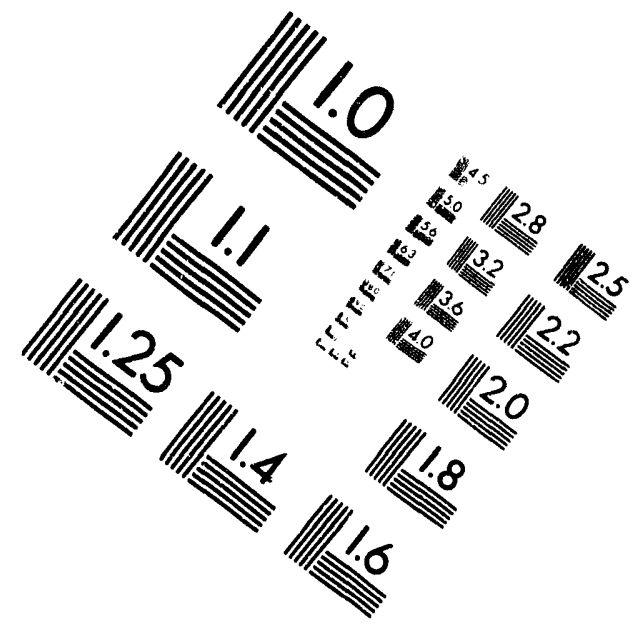

\title{
Centimeter
}

$\begin{array}{llllllllllllllll}1 & 2 & 3 & 4 & 5 & 6 & 7 & 8 & 9 & 10 & 11 & 12 & 13 & 14 & 15 & \mathrm{~mm}\end{array}$ Lш

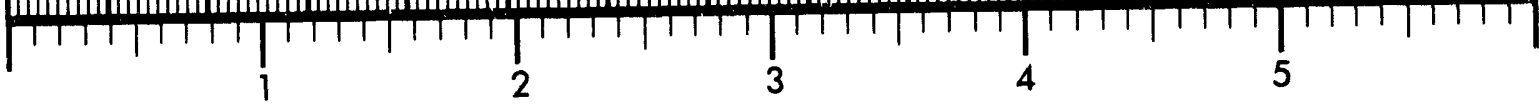
Inches
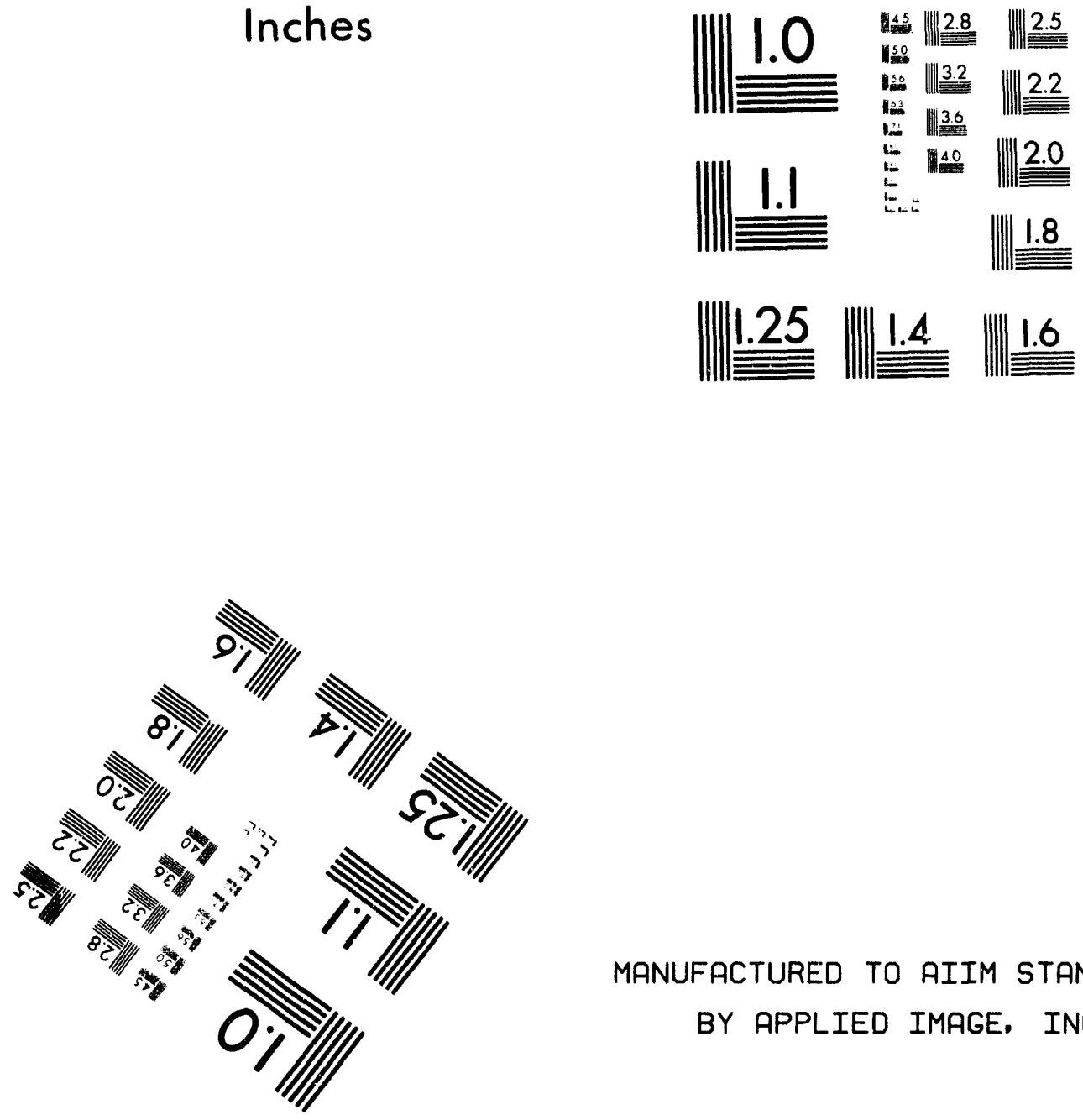

MANUFACTURED TO AIIM STANDARDS BY APPLIED IMAGE, INC.

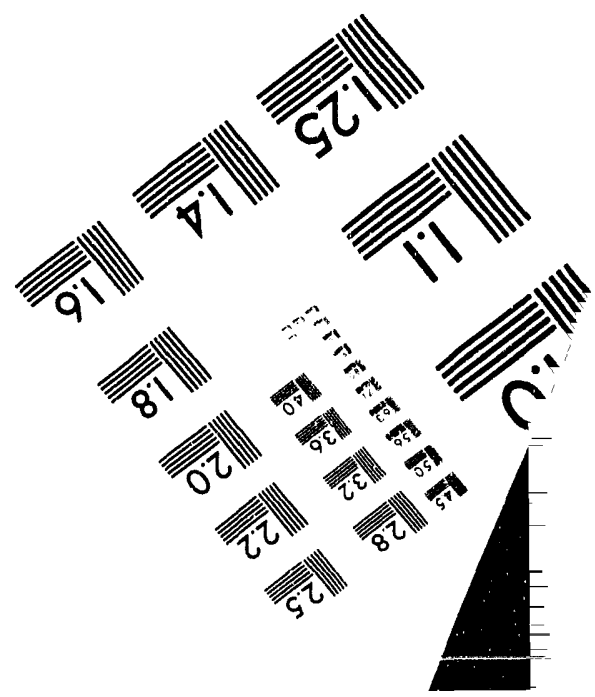



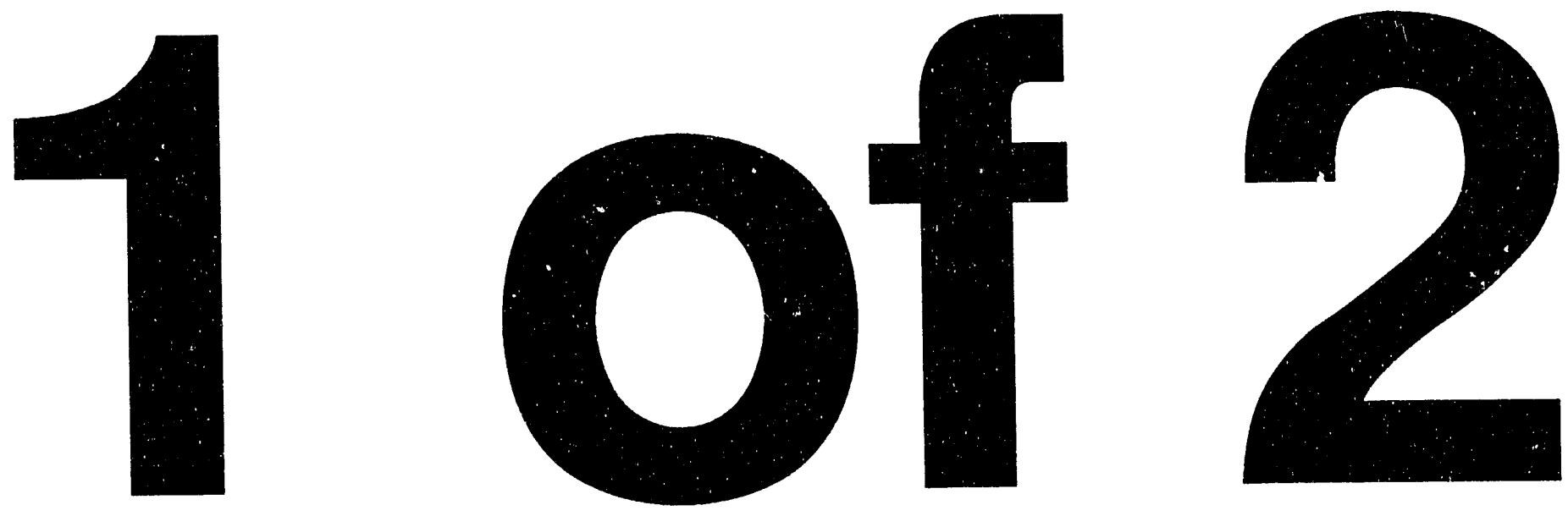


\section{PROCESSED SEISMIC MOTION RECORDS FROM \\ LANDERS, CALIFORNIA \\ EARTHQUAKE OF JUNE 28, 1992 \\ RECORDED AT SEISMOGRAPH STATIONS \\ IN SOUTHERN NEVADA}

April 1993

Peter K. Lum

Kenneth K. Honda

Prepared for

The U.S. Department of Energy

Nevada Operations Office

Under Contract DE-AC08-89NV10733

Prepared by

URS/John A. Blume \& Associates, Engineers

100 California Street, San Francisco, CA 94111

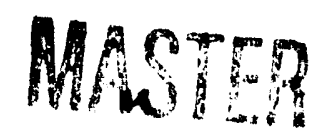




\section{LANDERS, CALIFORNIA \\ EARTHQUAKE OF 28 JUNE 1992}

\section{Background}

As part of the contract with the U.S. Department of Energy, Nevada Operations Office (DOE/NV), URS/John A. Blume \& Associates, Engineers (URS/Blume) maintained a network of seismographs in southern Nevada to monitor the ground motion generated by the underground nuclear explosions (UNEs) at the Nevada Test Site (NTS). The seismographs were located in the communities surrounding the NTS and the Las Vegas valley. When these seismographs were not used for monitoring the UNE generated motions, a limited number of them were maintained for monitoring motion generated by other than UNEs (e.g., motion generated by earthquakes, wind, blast). During the Landers earthquake of June 28, 1992, the systems located at 19 of these ground locations recorded the earthquake motions. Due to the potential benefit of these data for the scientific community, DOE/NV authorized the release of these records.

\section{Earthquake Parameters}

The earthquake parameters obtained from various sources were:

$\begin{array}{ll}\text { Epicenter: } & 34.217^{\circ} \mathrm{N}, 116.433^{\circ} \mathrm{W} \\ \text { Depth: } & 9 \mathrm{Km}(\mathrm{Caltech} / \mathrm{USGS}) \\ \text { Magnitude: } & 7.5 \mathrm{M}_{\mathrm{s}} \text { (NEIC) } \\ \text { Date: } & 28 \mathrm{June} 1992 \\ \text { Origin time: } & \text { Day } 180 \mathrm{Hr} 11 \text { Min } 57 \mathrm{Sec} 34 \text { (GMT) } \\ \text { Local time: } & 4: 58 \mathrm{a} . \mathrm{m} . \text { (PDT) }\end{array}$

The earthquake was felt by many of the people in southern Nevada. The motion was most perceptable by the occupants of high-rise buildings in Las Vegas; located approximately $250 \mathrm{~km}$ from the epicenter. There were isolated reports of some damage and power failure.

The Landers was followed by the Big Bear earthquake about 3 hours later with magnitude 6.5 and the next day by the magnitude 5.6 Skull Mountain earthquake near the NTS. Separate reports on the records obtained from these earthquakes were issued.

\section{Description of Equipment}

Three types of seismographs were used in the monitoring program. They were L-7 velocity meter manufactured by the Precision Instrument Company (L-7B system) and by Develco, Inc. (L-7D system); PDR-1 by Kinemetrics; and PDAS-100 by Teledyne Geotech. The FBA-11 accelerometers manufactured by Kinemetrics were utilized as sensors for both the PDR-1 and PDAS-100 recorders. The FBA-11 has a flat response from DC to $50 \mathrm{~Hz}$.

L-7 Velocity Meter: It is a continuously recording velocity seismograph which records the motion on an analog magnetic tape. The system has a flat velocity response over a frequency spectrum 
from $0.1 \mathrm{~Hz}$ to $34 \mathrm{~Hz}$ (Reference 1). It has a recording capability of 5 continuous days.

PDR-1: The acceleration signal from the sensors is converted to a digital form and recorded on a digital cassette tape. The selectable sampling rate was set at 200 samples per second per channel. The system is set to trigger on a short-term average (STA) and long-term average (LTA) threshold. It has a pre-event memory of about 5 seconds and has a recording capability of approximately 20 minutes.

PDAS-100: The acceleration signal from the sensors is converted to a digital form and recorded on a random access memory (RAM) within the system. The selectable sampling rate was set at 200 samples per second per channel. The system is set to trigger on a short-term average (STA) and long-term average (LTA) threshold. It has a selectable pre-event memory of up to 20 seconds and has a recording capability of approximately 20 minutes.

\section{Seismic Recordings}

The subject earthquake was recorded at 19 ground station sites in southern Nevada as shown on Figure 1. Those in the Las Vegas valley are detailed in Figure 2. Table 1 tabulates the system type, location, geodetic coordinates and distance from epicenter for each station. At several selected sites, the station was monitored by a secondary system. When both systems recorded the motion, the systems are designated $\mathrm{A}$ and $\mathrm{B}$ on the tables.

\section{Conversion and Processing}

The records obtained from the earthquake were retrieved and converted from field data to a computer compatible format for processing. The processing was performed using URS/Blume's standard signal processing as described in Reference 2.

Briefly, the processing was performed in three phases:

- In Phase 1 processing, the seismic signal was converted from field data to a computer compatible format using a special interface equipment. The data were scaled to an engineering unit $\left(\mathrm{cm} / \mathrm{sec}^{2}\right.$ or $\left.\mathrm{cm} / \mathrm{sec}\right)$. The resulting plots were checked for any error and reviewed for additional processing requirements.

- In Phase 2 processing, the records were processed using the noise reduction techniques. The techniques include baseline correction, cosine tapering and application of low-pass and highpass filtering using the Butterworth filter, Order 4.

For the UNE generated ground motion records, they are generaily band-pass filtered from 0.1 to $33 \mathrm{~Hz}$. The pseudo relative velocity spectrum (PSRV) of 49 periods and 5 percent of critical damping is computed. The 49 periods are equally spaced on a logarithmic scale from 0.05 to 6.072 seconds. Fourier amplitude spectra are generated before and after the Phase 2 processing. The corrected acceleration, velocity and displacement time histories are generated using numerical differentiation and integration. 
During the preliminary processing of records from distant earthquake,s, it was observed that these records appeared to contain motion of longer period than those observed from the UNE generated ground motion. Thus the processing procedure using the standard filter cut-offs would partially filter-out this long period motion. In order to examine the characteristics of the long-period motion, the lower cut-off frequency was extended to $0.05 \mathrm{~Hz}$ and the PSRV was extended to a period of 30.11 seconds. Of course, the PSRV values for periods greater than the cut-off frequency or the beyond the recording system response are not the optimum values.

- In the final phase, the records were arranged for the long-term preservation and for report preparation. The final product contains data plots in chronological order of data processing; original recording, uncorrected and corrected Fourier amplitude spectra, corrected acceleration, velocity, displacement and PSRVs.

\section{Results}

Table 2 tabulates the uncorrected peak acceleration or velocity, corrected peak acceleration, velocity and displacement values and any data processing comments. The data are written in a format which are compatible with the standard format for the California Strong-Motion Instrumentation Program (CSMIP) tapes [Reference 3]. Table A summaries the standard data format.

The data were copied on to a 8MM tape using ASCII format (record length $=80$, blocksize $=2000$ ). Table B gives examples of VAX/VMS commands used to create the data tape and an instruction for retrieval of the data files.

\section{Acknowledgment}

The network of seismographs in southern Nevada to monitor the seismic motion generated by the UNEs at the NTS was maintained by the staff of electronic technicians consisting of Ray Bradley, Don Morgan, Robert Stewart, Lori Forrester and Jim Graham. The seismic records conversion and processing staff included Vickie Ross, Stephanie Miller, Rosemarie Musso, Ben Gacula and Cory Lieber.

\section{References}

1. Navarro, R., and G.M. Wuollet, "The L-7 Velocity Seismograph Shaking-Table Results", NOAA Technical Report ERL 254-ESL 26, Boulder, Colorado, Juyy 1972.

2. Lum, P. K., "Overview of Seismic Signal Conversion and Processing Operations", URS/John A. Blume \& Associates, Engineers, San Francisco, JAB-10733-TM3, March 1991.

3. Shakal, A. F. and Huang, M. J., "Standard Tape Format for CSMIP Strong-Motion Data Tapes", California Department of Conservation, Division of Mines and Geology, Office of Strong Motion Studies, Report OSMS 85-03, December 1985. 


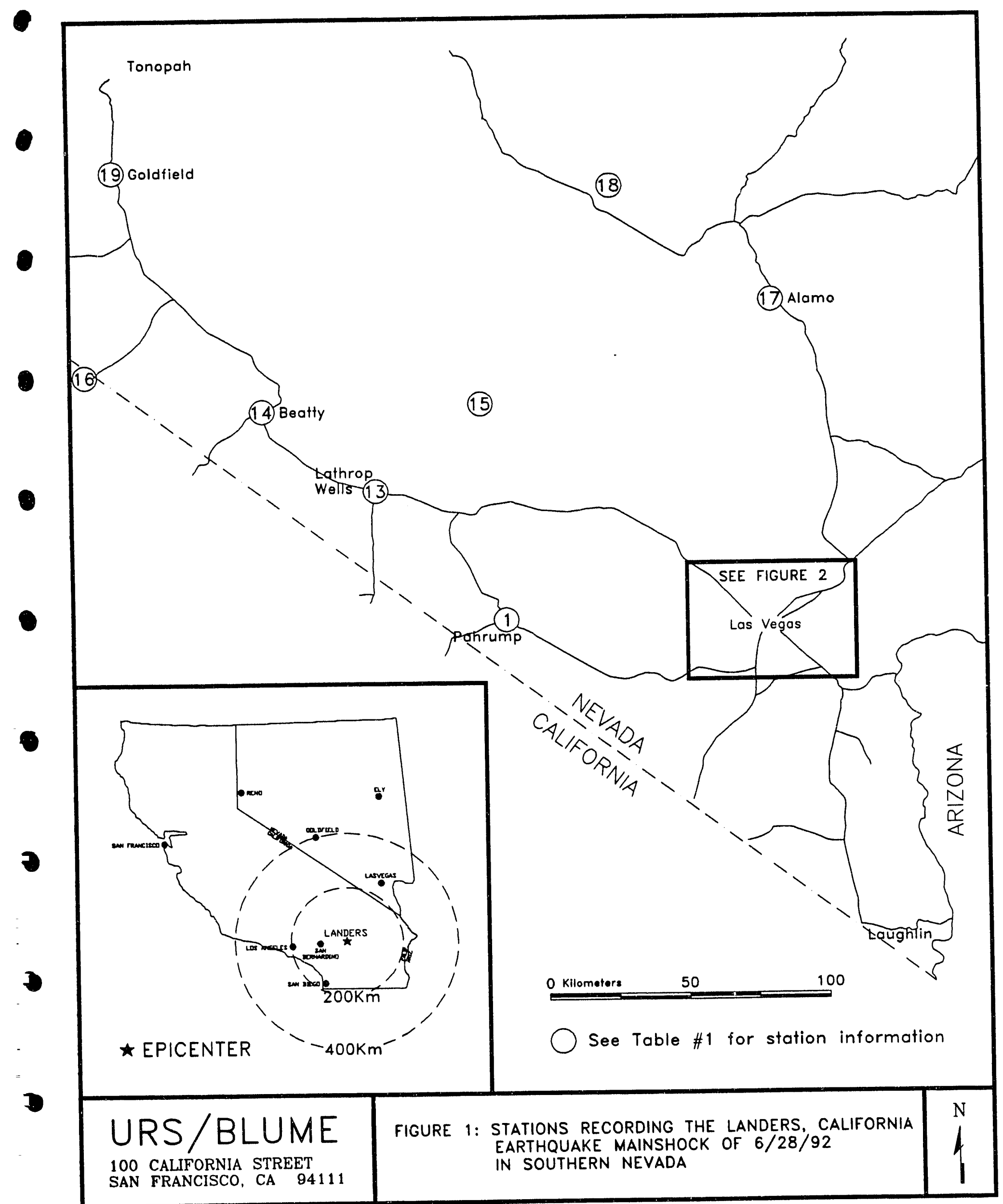




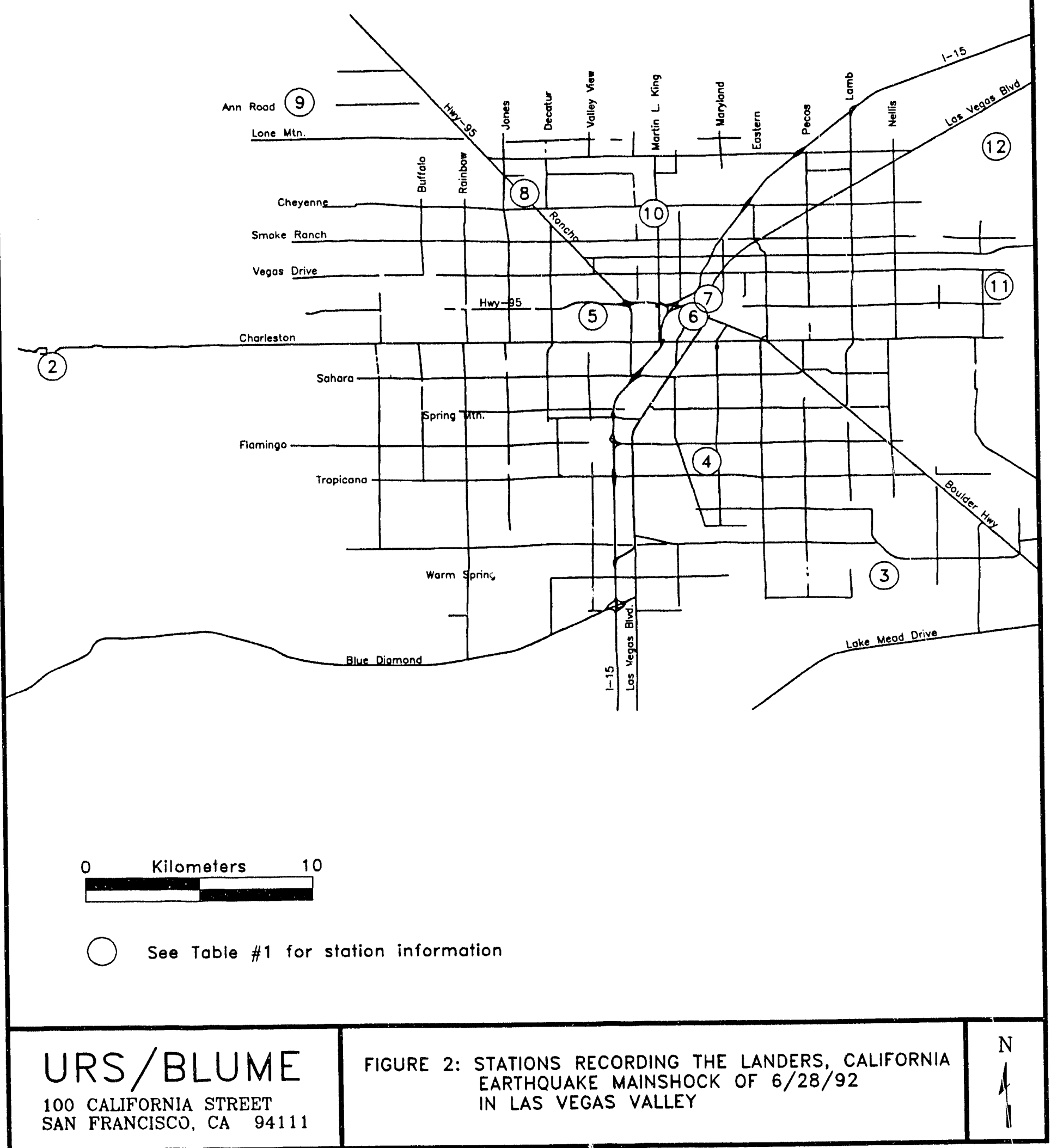




\section{TABLE 1: LOCATIONS OF THE RECORDING STATIONS}

\begin{tabular}{|c|c|c|c|c|c|}
\hline $\begin{array}{l}\text { Station } \\
\text { No. }\end{array}$ & $\begin{array}{l}\text { System } \\
\text { Iype }\end{array}$ & Location & Coordina & Ees & $\begin{array}{l}\text { Distance from } \\
\text { Epicenter }(\mathrm{Km})\end{array}$ \\
\hline 1 & PDR-1 & Pahrump & $115.98 \mathrm{~W}$ & $36.21 \mathrm{~N}$ & 225 \\
\hline 2 & PDR-1 & Las Vegas & $115.41 W$ & $36.15 \mathrm{~N}$ & 234 \\
\hline 3 & PDR-1 & Las Vegas & $115.07 W$ & $36.06 \mathrm{~N}$ & 239 \\
\hline 4 & PDR-1 & Las Vegas & $115.14 \mathrm{~W}$ & $36.11 \mathrm{~N}$ & 241 \\
\hline 5 & PDR-1 & Las Vegas & $115.19 \mathrm{~W}$ & $36.17 N$ & 245 \\
\hline 6 & PDR-1 & Las Vegas & $115.14 W$ & $36.17 \mathrm{~N}$ & 247 \\
\hline 7 & PDR-1 & Las Vegas & $115.14 \mathrm{~W}$ & $36.17 \mathrm{~N}$ & 247 \\
\hline 8 & PDR-1 & Las Vegas & $115.22 \mathrm{~W}$ & $36.22 N$ & 249 \\
\hline 9 & PDR-1 & Las Vegas & $115.31 \mathrm{~W}$ & $36.26 \mathrm{~N}$ & 249 \\
\hline 10 & PDR-1 & Las Vegas & $115.16 \mathrm{~W}$ & $36.21 N$ & 250 \\
\hline 11 & PDR-1 & Las Vegas & $115.02 \mathrm{~W}$ & $36.18 \mathrm{~N}$ & 253 \\
\hline 12 & PDAS-100 & Las Vegas & $115.02 \mathrm{~W}$ & $36.24 \mathrm{~N}$ & 259 \\
\hline $\begin{array}{l}13 \mathrm{~A} \\
13 \mathrm{~B}\end{array}$ & $\begin{array}{l}\text { PDR-1 } \\
\text { PDAS-100 }\end{array}$ & Lathrop Wella & $116.40 \mathrm{~W}$ & $36.64 N$ & 270 \\
\hline 14 & PDR-1 & Beatty & $116.76 \mathrm{~W}$ & $36.91 \mathrm{~N}$ & 301 \\
\hline $\begin{array}{l}15 A \\
15 B\end{array}$ & $\begin{array}{l}\text { PDR-1 } \\
\text { PDAS- } 100\end{array}$ & Nevada Test Site & $116.06 \mathrm{~W}$ & $36.93 N$ & 304 \\
\hline 16 & PDR-1 & Death Valley & $117.34 \mathrm{~W}$ & $37.03 N$ & 323 \\
\hline $\begin{array}{l}17 \mathrm{~A} \\
17 \mathrm{~B}\end{array}$ & $\begin{array}{l}\text { PDR-1 } \\
\text { PDAS-100 }\end{array}$ & Alamo & $115.12 \mathrm{~W}$ & $37.27 \mathrm{~N}$ & 359 \\
\hline 18 & PDR-1 & Tempiute & $115.63 W$ & $37.65 \mathrm{~N}$ & 388 \\
\hline 19 & PDR-1 & Goldfield & $117.23 \mathrm{~W}$ & $37.71 \mathrm{~N}$ & 395 \\
\hline
\end{tabular}


TABLE 2: PEAK AMPLITUDES FROM LANDERS EARTHQUAKE OF 6/28/92

\begin{tabular}{|c|c|c|c|c|c|c|c|}
\hline \multicolumn{2}{|c|}{ Station } & \multicolumn{2}{|c|}{$\begin{array}{rr}<- \text { Uncorrected }-> \\
\text { Accel. Velocity } \\
1 \mathrm{~cm} / \mathrm{sec}^{2} & (\mathrm{~cm} / \mathrm{sec})\end{array}$} & $\begin{array}{r}\text { Accel. } \\
1 \mathrm{~cm} / \mathrm{sec}^{2}\end{array}$ & $\begin{array}{l}\text { Corrected } \\
\text { Velocity } \\
(\mathrm{cm} / \mathrm{sec})\end{array}$ & $\begin{array}{c}\text { Disp } \\
(\mathrm{cm})\end{array}$ & Comments \\
\hline $\begin{array}{l}1 \\
1 \\
1\end{array}$ & $\begin{array}{l}\text { DOWN } \\
\text { NORTH } \\
\text { WEST }\end{array}$ & $\begin{array}{r}6.601 \\
-14.414 \\
-14.373\end{array}$ & $\begin{array}{l}0.000 \\
0.000 \\
0.000\end{array}$ & $\begin{array}{r}6.538 \\
-14.438 \\
-14.459\end{array}$ & $\begin{array}{l}-2.451 \\
-4.277 \\
-5.960\end{array}$ & $\begin{array}{r}-3.336 \\
4.362 \\
7.201\end{array}$ & \\
\hline $\begin{array}{l}2 \\
2 \\
2\end{array}$ & $\begin{array}{l}\text { DOWN } \\
\text { NORTH } \\
\text { WEST }\end{array}$ & $\begin{array}{r}-11.015 \\
-15.001 \\
10.513\end{array}$ & $\begin{array}{l}0.000 \\
0.000 \\
0.000\end{array}$ & $\begin{array}{l}-9.262 \\
12.767 \\
10.916\end{array}$ & $\begin{array}{r}2.173 \\
-1.875 \\
4.492\end{array}$ & $\begin{array}{r}-3.765 \\
-2.977 \\
6.144\end{array}$ & \\
\hline $\begin{array}{l}3 \\
3 \\
3\end{array}$ & $\begin{array}{l}\text { DOWN } \\
\text { NORTH } \\
\text { WEST }\end{array}$ & $\begin{array}{l}11.856 \\
17.178 \\
17.603\end{array}$ & $\begin{array}{l}0.000 \\
0.000 \\
0.000\end{array}$ & $\begin{array}{l}11.877 \\
17.129 \\
17.708\end{array}$ & $\begin{array}{r}1.923 \\
-3.122 \\
6.273\end{array}$ & $\begin{array}{r}-1.713 \\
2.579 \\
-3.888\end{array}$ & \\
\hline $\begin{array}{l}4 \\
4 \\
4\end{array}$ & $\begin{array}{l}\text { DOWN } \\
\text { NORTH } \\
\text { WEST }\end{array}$ & $\begin{array}{r}8.452 \\
20.690 \\
13.245\end{array}$ & $\begin{array}{l}0.000 \\
0.000 \\
0.000\end{array}$ & $\begin{array}{r}8.497 \\
20.640 \\
13.347\end{array}$ & $\begin{array}{l}2.418 \\
4.319 \\
5.397\end{array}$ & $\begin{array}{l}-2.297 \\
-3.202 \\
-4.294\end{array}$ & \\
\hline $\begin{array}{l}5 \\
5 \\
5\end{array}$ & $\begin{array}{l}\text { DOWN } \\
\text { NORTH } \\
\text { WEST }\end{array}$ & $\begin{array}{r}-10.469 \\
27.442 \\
-21.626\end{array}$ & $\begin{array}{l}0.000 \\
0.000 \\
0.000\end{array}$ & $\begin{array}{r}-10.449 \\
27.357 \\
-21.555\end{array}$ & $\begin{array}{l}3.826 \\
4.660 \\
7.574\end{array}$ & $\begin{array}{r}-2.678 \\
3.282 \\
5.269\end{array}$ & \\
\hline $\begin{array}{l}6 \\
6 \\
6\end{array}$ & $\begin{array}{l}\text { DOWN } \\
\text { NORTH } \\
\text { WEST }\end{array}$ & $\begin{array}{r}11.183 \\
19.875 \\
-24.478\end{array}$ & $\begin{array}{l}0.000 \\
0.000 \\
0.000\end{array}$ & $\begin{array}{r}11.129 \\
19.938 \\
-24.261\end{array}$ & $\begin{array}{r}3.462 \\
-7.280 \\
9.219\end{array}$ & $\begin{array}{r}-2.329 \\
3.311 \\
6.445\end{array}$ & \\
\hline $\begin{array}{l}7 \\
7 \\
7\end{array}$ & $\begin{array}{l}\text { DOWIN } \\
\text { NORTH } \\
\text { WEST }\end{array}$ & $\begin{array}{r}9.198 \\
-20.241 \\
-22.702\end{array}$ & $\begin{array}{l}0.000 \\
0.000 \\
0.000\end{array}$ & $\begin{array}{r}9.158 \\
-20.163 \\
-22.605\end{array}$ & $\begin{array}{r}2.874 \\
-6.761 \\
8.899\end{array}$ & $\begin{array}{r}-2.320 \\
3.745 \\
6.707\end{array}$ & \\
\hline $\begin{array}{l}8 \\
8 \\
8\end{array}$ & $\begin{array}{l}\text { DOWN } \\
\text { NORTH } \\
\text { WEST }\end{array}$ & $\begin{array}{r}-7.352 \\
18.683 \\
-21.134\end{array}$ & $\begin{array}{l}0.000 \\
0.000 \\
0.000\end{array}$ & $\begin{array}{r}-7.393 \\
18.580 \\
-20.756\end{array}$ & $\begin{array}{r}3.193 \\
-3.204 \\
5.538\end{array}$ & $\begin{array}{r}-3.052 \\
2.471 \\
4.651\end{array}$ & \\
\hline $\begin{array}{l}9 \\
9 \\
9\end{array}$ & $\begin{array}{l}\text { DOWN } \\
\text { NORTH } \\
\text { WEST }\end{array}$ & $\begin{array}{r}-6.688 \\
10.959 \\
-12.589\end{array}$ & $\begin{array}{l}0.000 \\
0.000 \\
0.000\end{array}$ & $\begin{array}{r}-6.677 \\
10.924 \\
-12.673\end{array}$ & $\begin{array}{l}2.615 \\
2.593 \\
3.810\end{array}$ & $\begin{array}{r}-3.022 \\
-2.819 \\
4.800\end{array}$ & \\
\hline $\begin{array}{l}10 \\
10 \\
10\end{array}$ & $\begin{array}{l}\text { DOWN } \\
\text { NORTH } \\
\text { WEST }\end{array}$ & $\begin{array}{r}10.107 \\
25.868 \\
-21.492\end{array}$ & $\begin{array}{l}0.000 \\
0.000 \\
0.000\end{array}$ & $\begin{array}{r}10.055 \\
25.950 \\
-21.561\end{array}$ & $\begin{array}{r}3.349 \\
5.099 \\
-6.611\end{array}$ & $\begin{array}{r}-3.215 \\
3.513 \\
4.924\end{array}$ & \\
\hline $\begin{array}{l}11 \\
11 \\
11\end{array}$ & $\begin{array}{l}\text { DOWN } \\
\text { NORTH } \\
\text { WEST }\end{array}$ & $\begin{array}{r}3.824 \\
6.423 \\
-7.320\end{array}$ & $\begin{array}{l}0.000 \\
0.000 \\
0.000\end{array}$ & $\begin{array}{r}3.799 \\
6.297 \\
-7.461\end{array}$ & $\begin{array}{r}1.431 \\
1.911 \\
-2.823\end{array}$ & $\begin{array}{r}-1.894 \\
-2.315 \\
3.204\end{array}$ & \\
\hline $\begin{array}{l}12 \\
12 \\
12\end{array}$ & $\begin{array}{l}\text { DOWN } \\
\text { NORTH } \\
\text { WEST }\end{array}$ & $\begin{array}{r}-11.635 \\
14.763 \\
20.470\end{array}$ & $\begin{array}{l}0.000 \\
0.000 \\
0.000\end{array}$ & $\begin{array}{r}-11.618 \\
14.748 \\
20.448\end{array}$ & $\begin{array}{r}2.690 \\
4.116 \\
-5.278\end{array}$ & $\begin{array}{r}-1.890 \\
-2.358 \\
3.828\end{array}$ & \\
\hline $\begin{array}{l}13 A \\
13 A \\
13 A\end{array}$ & $\begin{array}{l}\text { DOWN } \\
\text { NORTH } \\
\text { WEST }\end{array}$ & $\begin{array}{l}-8.168 \\
14.805 \\
15.097\end{array}$ & $\begin{array}{l}0.000 \\
0.000 \\
0.000\end{array}$ & $\begin{array}{l}-8.223 \\
14.722 \\
14.937\end{array}$ & $\begin{array}{r}-3.705 \\
5.498 \\
8.987\end{array}$ & $\begin{array}{r}-4.060 \\
-5.316 \\
8.988\end{array}$ & \\
\hline $\begin{array}{l}13 B \\
13 B \\
13 B\end{array}$ & $\begin{array}{l}\text { DOWN } \\
\text { NORTH } \\
\text { WEST }\end{array}$ & $\begin{array}{l}-8.446 \\
14.814 \\
15.370\end{array}$ & $\begin{array}{l}0.000 \\
0.000 \\
0.000\end{array}$ & $\begin{array}{l}-8.469 \\
14.769 \\
15.179\end{array}$ & $\begin{array}{l}3.620 \\
5.537 \\
9.131\end{array}$ & $\begin{array}{r}-4.146 \\
-5.212 \\
9.356\end{array}$ & \\
\hline
\end{tabular}


TABLE 2: PEAK AMPLITUDES FROM LANDERS EARTHQUAKE OF 6/28/92

\begin{tabular}{|c|c|c|c|c|c|c|c|}
\hline \multicolumn{2}{|c|}{ station } & $\begin{array}{c}<-- \text { Uncorr } \\
\text { Accel } \\
1 \mathrm{~cm} / \mathrm{sec}^{2}\end{array}$ & $\begin{array}{l}\text { ected } \rightarrow-> \\
\text { velocity } \\
(\mathrm{cm} / \mathrm{sec})\end{array}$ & $\begin{array}{r}\text { Accel. } \\
1 \mathrm{~cm} / \mathrm{sec}^{2}\end{array}$ & $\begin{array}{l}\text { Corrected - } \\
\text { Velocity } \\
(\mathrm{cm} / \mathrm{sec})\end{array}$ & $\begin{array}{l}\text { Disp } \\
(\mathrm{cm})\end{array}$ & Comments \\
\hline $\begin{array}{l}14 \\
14 \\
14\end{array}$ & $\begin{array}{l}\text { DOWN } \\
\text { NORTH } \\
\text { WEST }\end{array}$ & $\begin{array}{r}7.511 \\
15.914 \\
-15.647\end{array}$ & $\begin{array}{l}0.000 \\
0.000 \\
0.000\end{array}$ & $\begin{array}{r}7.284 \\
15.859 \\
-15.728\end{array}$ & $\begin{array}{l}1.776 \\
1.920 \\
4.297\end{array}$ & $\begin{array}{r}-2.580 \\
-2.661 \\
5.486\end{array}$ & \\
\hline $\begin{array}{l}15 A \\
15 A \\
15 A\end{array}$ & $\begin{array}{l}\text { DOWN } \\
\text { NORTH } \\
\text { WEST }\end{array}$ & $\begin{array}{r}-3.585 \\
6.130 \\
5.928\end{array}$ & $\begin{array}{l}0.000 \\
0.000 \\
0.000\end{array}$ & $\begin{array}{r}-3.602 \\
6.133 \\
5.945\end{array}$ & $\begin{array}{r}2.477 \\
-3.665 \\
-3.343\end{array}$ & $\begin{array}{r}-3.097 \\
-4.611 \\
4.884\end{array}$ & \\
\hline $\begin{array}{l}15 B \\
15 B \\
15 B\end{array}$ & $\begin{array}{l}\text { DOWN } \\
\text { NORTH } \\
\text { WEST }\end{array}$ & $\begin{array}{r}-3.558 \\
6.199 \\
6.035\end{array}$ & $\begin{array}{l}0.000 \\
0.000 \\
0.000\end{array}$ & $\begin{array}{r}-3.522 \\
6.172 \\
6.016\end{array}$ & $\begin{array}{r}2.391 \\
-3.699 \\
-3.442\end{array}$ & $\begin{array}{r}-2.926 \\
-4.698 \\
4.946\end{array}$ & \\
\hline $\begin{array}{l}16 \\
16 \\
16\end{array}$ & $\begin{array}{l}\text { DOWN } \\
\text { NORTH } \\
\text { WEST }\end{array}$ & $\begin{array}{r}-3.170 \\
-5.902 \\
6.104\end{array}$ & $\begin{array}{l}0.000 \\
0.000 \\
0.000\end{array}$ & $\begin{array}{r}-3.115 \\
-5.840 \\
6.198\end{array}$ & $\begin{array}{r}1.773 \\
-2.494 \\
6.759\end{array}$ & $\begin{array}{r}1.754 \\
-3.647 \\
9.431\end{array}$ & \\
\hline $\begin{array}{l}17 A \\
17 A \\
17 A\end{array}$ & $\begin{array}{l}\text { DOWN } \\
\text { NORTH } \\
\text { WEST }\end{array}$ & $\begin{array}{l}3.685 \\
6.003 \\
6.917\end{array}$ & $\begin{array}{l}0.000 \\
0.000 \\
0.000\end{array}$ & $\begin{array}{l}3.695 \\
5.940 \\
6.653\end{array}$ & $\begin{array}{r}1.345 \\
-2.488 \\
-3.803\end{array}$ & $\begin{array}{r}1.579 \\
-1.869 \\
-3.805\end{array}$ & \\
\hline $\begin{array}{l}17 \mathrm{~B} \\
17 \mathrm{~B} \\
17 \mathrm{~B}\end{array}$ & $\begin{array}{l}\text { DOWN } \\
\text { NORTH } \\
\text { WEST }\end{array}$ & $\begin{array}{r}3.632 \\
-5.513 \\
7.070\end{array}$ & $\begin{array}{l}0.000 \\
0.000 \\
0.000\end{array}$ & $\begin{array}{r}3.667 \\
-5.479 \\
7.084\end{array}$ & $\begin{array}{r}1.283 \\
-1.996 \\
-3.915\end{array}$ & $\begin{array}{r}1.624 \\
-1.526 \\
-4.043\end{array}$ & \\
\hline $\begin{array}{l}18 \\
18 \\
18\end{array}$ & $\begin{array}{l}\text { DOWN } \\
\text { NORTH } \\
\text { WEST }\end{array}$ & $\begin{array}{r}1.678 \\
1.508 \\
-2.181\end{array}$ & $\begin{array}{l}0.000 \\
0.000 \\
0.000\end{array}$ & $\begin{array}{r}1.458 \\
1.484 \\
-2.173\end{array}$ & $\begin{array}{r}-1.399 \\
1.653 \\
-2.638\end{array}$ & $\begin{array}{r}-1.866 \\
2.006 \\
4.001\end{array}$ & LATE TRIGGERED \\
\hline $\begin{array}{l}19 \\
19 \\
19\end{array}$ & $\begin{array}{l}\text { DOWN } \\
\text { NORTH } \\
\text { WEST }\end{array}$ & $\begin{array}{r}-1.460 \\
-3.708 \\
3.062\end{array}$ & $\begin{array}{l}0.000 \\
0.000 \\
0.000\end{array}$ & $\begin{array}{r}-1.427 \\
-3.586 \\
3.034\end{array}$ & $\begin{array}{r}1.562 \\
-4.479 \\
-2.074\end{array}$ & $\begin{array}{r}2.020 \\
-6.627 \\
2.456\end{array}$ & \\
\hline
\end{tabular}




\section{TABLE 3: DATA FORMAT}

Each station contains 3 data channels and contains the following 4 files:

$\begin{array}{lll}\text { Vol } 1 \text { Uncorrected acceleration } & \text { Units } \\ \text { vol } 2 \text { Corrected acceleration } & 1000 / \mathrm{g} \\ & \mathrm{cm} \star 100 / \mathrm{sec}^{2} \\ \text { Corrected velocity } & \mathrm{cm} \star 100 / \mathrm{sec} \\ \text { Vol } 3 \text { Psudo Response Velocity } & \mathrm{cm} \star 100 \\ \text { Vol } 4 \text { Fourier Amplitude Spectra } & \mathrm{cm} / \mathrm{sec} \\ \end{array}$

A typical station contains the following data:

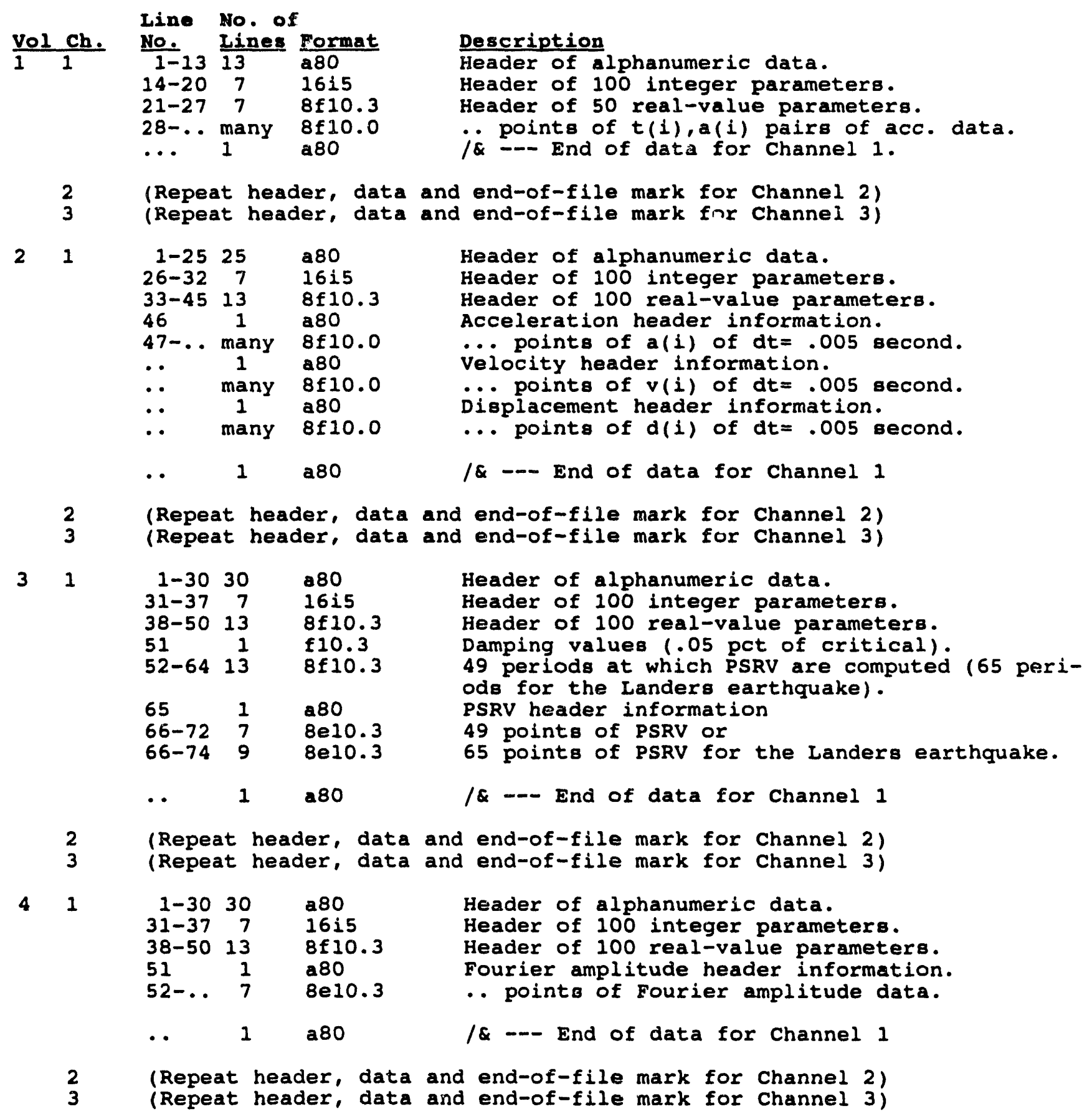


TABLE 4: READ / WRITE DATA ON 8MM ASCII FORMATTED TAPE

\section{WRITE DATA ON ASCII FORMATTED 8MA TAPE}

Below is an example to save files on $8 \mathrm{MM}$ ASCII formatted tape with record length $=80$, block size $=2000$ using the VAX/VMS operating system utilities TWRITE:

\$ INIT MUBO: 0705B

$\$$ MOUNT/NOASSIST/FOR/RECORD $=80 / B L O C K=2000$ MUBO:

$\$$ TWRITE/ASCII / RECORD $=80 /$ BLOCK $=2000 / O U T P U T=M U B O:$

\$ TWRITE/ASCII/RECORD $=80 /$ BLOCK=2000/OUTPUT $=$ MUE?:

$\$$ TWRITE/ASCII/RECORD $=80 /$ BLOCK $=2000 /$ OUTPUT $=$ MUBO:

$\$$ TWRITE/ASCII/RECORD $=80 / \mathrm{BLOCK}=2000 /$ OUTPUT $=M U B O$ :

$\$$ TWRITE/ASCII/RECORD $=80 / \mathrm{BLOCK}=2000 /$ OUTPUT=MUBO:

$\$$ TWRITE/ASCII / RECORD $=80 /$ BLOCK $=2000 /$ OUTPUT $=$ MUBO:

$\$$ TWRITE/ASCII/RECORD $=80 / \mathrm{BLOCK}=2000 /$ OUTPUT $=M U B O:$

$\$$ TWRITE/ASCII/RECORD $=80 / B L O C K=2000 / O U T P U T=M U B O:$

STATN1.V1

STATN1.V2

STATN $1 . V 3$

STATN1.V4

STATN2.V1

STATN2 . V2

STATN2.V3

... etc.. (for all stations, each with v1,v2,v3 and v4 data)

\$ EXIT

\section{READ/RETRIEVE DATA FROM ASCII FORMATTED BMM TAPE}

Below is an example to read/retrieve files from an 8MM ASCII formatted tape with record length $=80$, block size $=2000$ using the vax/VMS operating system utilities TREAD:

\$ MOUNT/NOASSIST/FOR/RECORD $=80 / \mathrm{BLOCK}=2000$ MUBO:

$\$ T R E A D / A S C I I / R E C O R D=80 / B L O C K=2000 /$ INPUT $=$ MUBO:

$\$$ TREAD /ASCII / RECORD $=80 / B L O C K=2000 /$ INPUT $=$ MUBO :

$\$$ TREAD/ASCII/RECORD $=80 /$ BLOCK $=2000 /$ INPUT $=$ MUBO:

S TREAD/ASCII /RECORD $=80 / \mathrm{BLOCK}=2000 /$ INPUT $=$ MUBO:

$\$$ TREAD $/$ ASCII $/$ RECORD $=80 /$ BLOCK $=2000 /$ INPUT $=M U B O:$

\$ TREAD/ASCII /RECORD $=80 /$ BLOCK $=2000 /$ INPUT $=$ MUBO:

$\$ T R E A D / A S C I I / R E C O R D=80 / B L O C K=2000 /$ INPUT $=M U B O:$

$\$$ TREAD/ASCII/RECORD $=80 /$ BLOCK $=2000 /$ INPUT $=$ MUBO:

STATN1.VI

STATN1.V2

STATN1.V3

STATN 1.V4

STATN2.V1

STATN2.V2

STATN2.V3

STATN2.V4

... etc.. (for all stations, each with v1,v2,v3 and v4 data)

\$ EXIT 


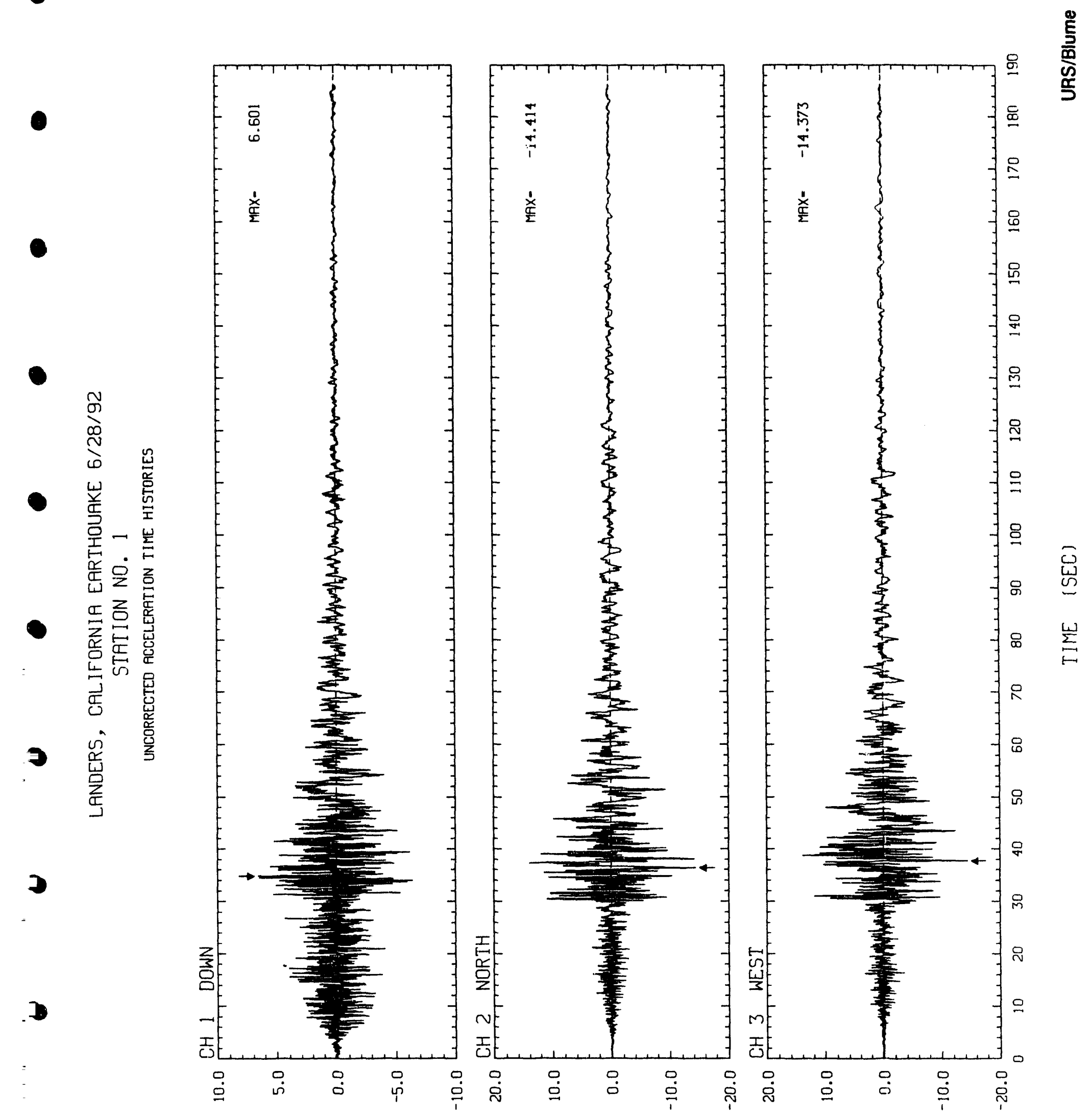

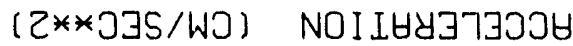



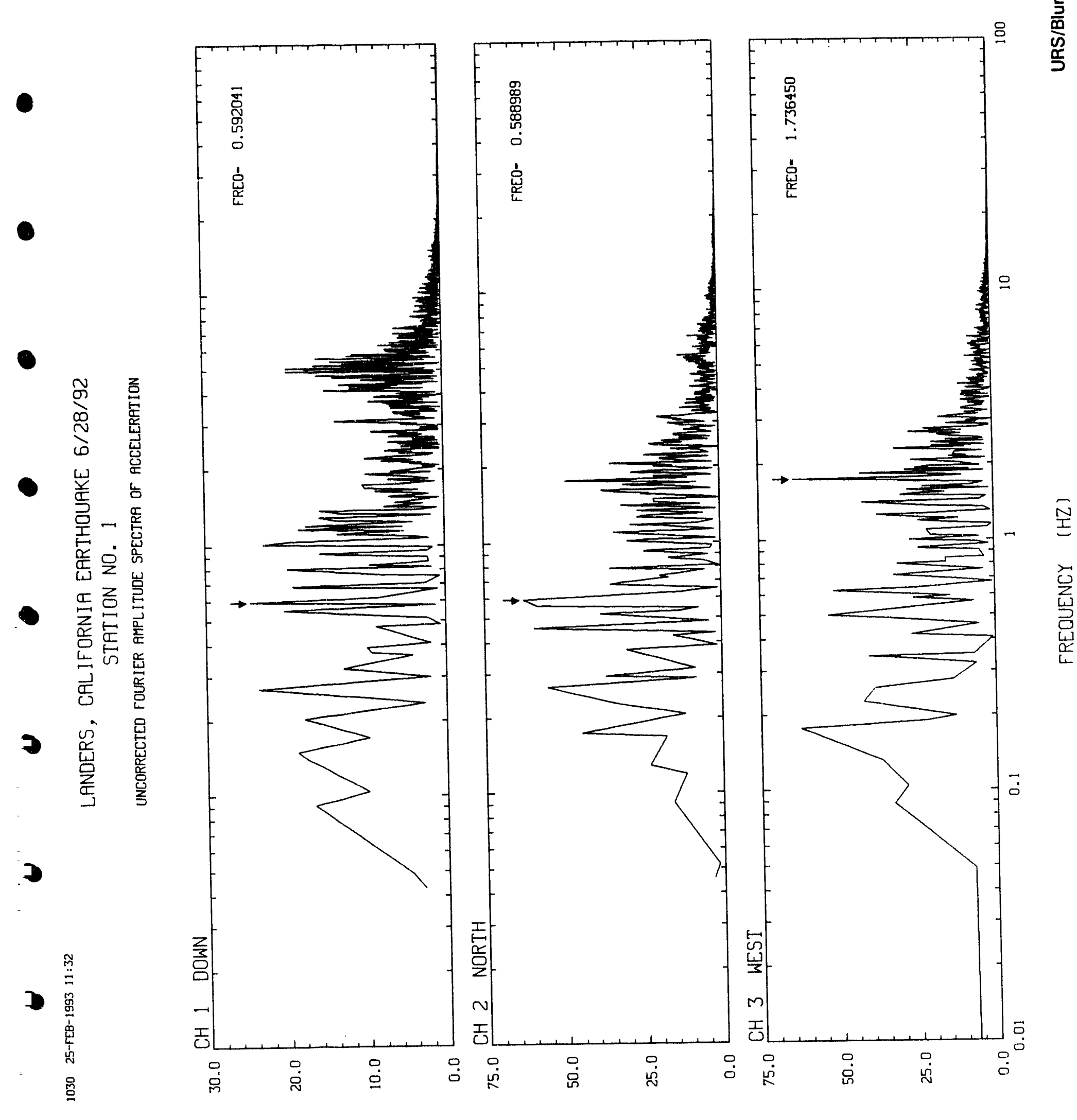

(03S/WJ) JOกLI J 


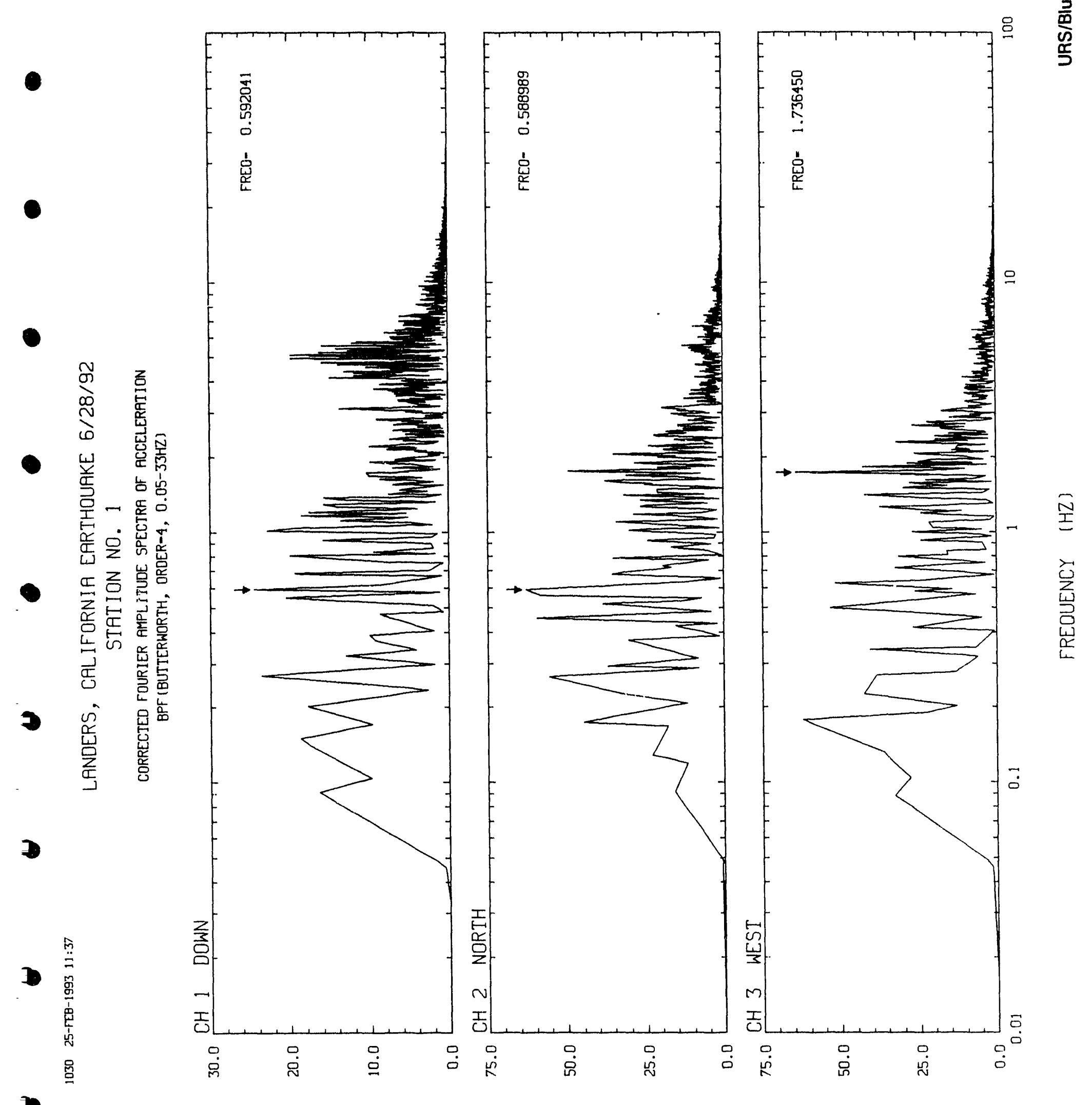

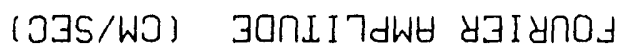




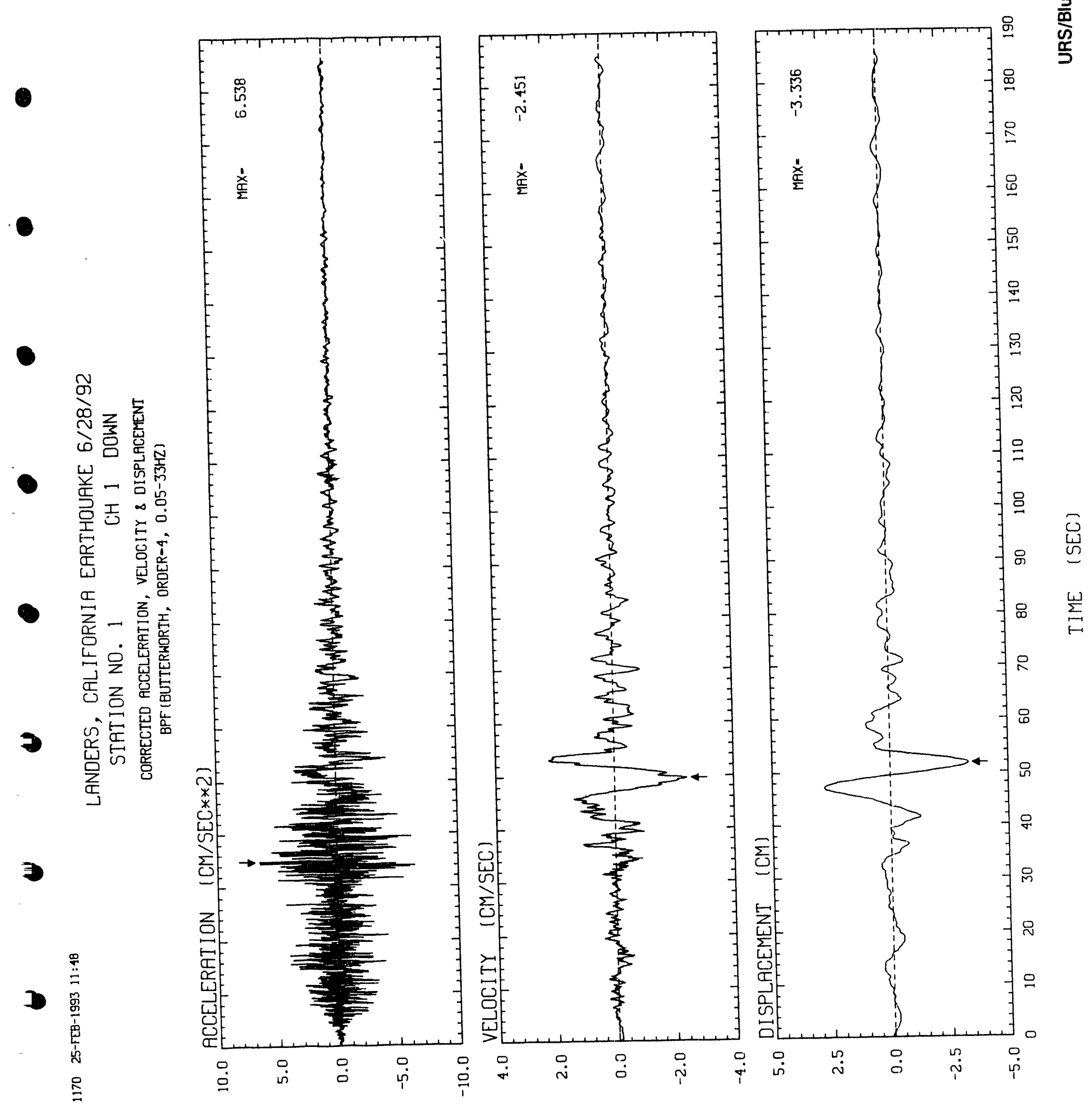




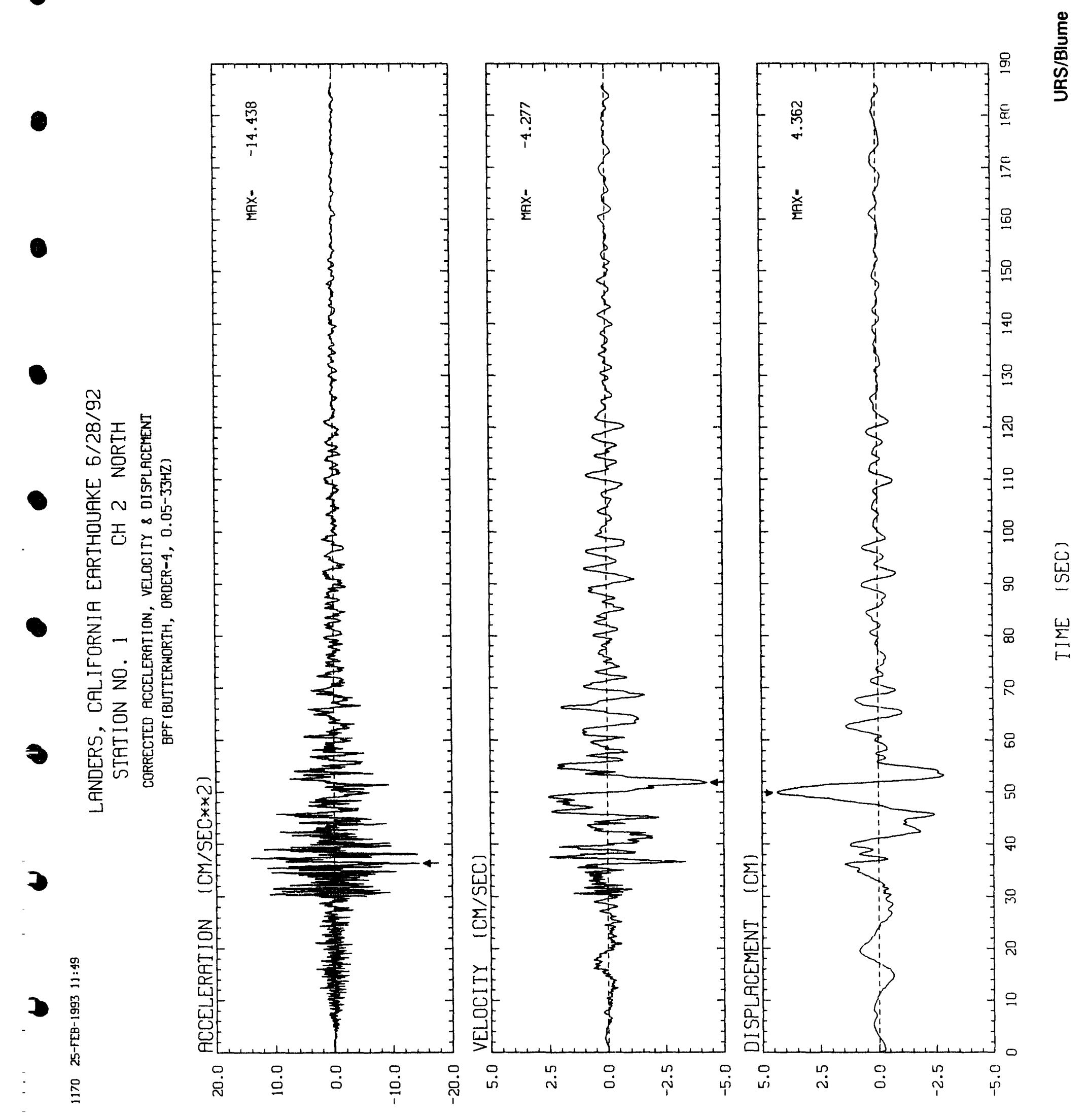

JOกI I 7dW 


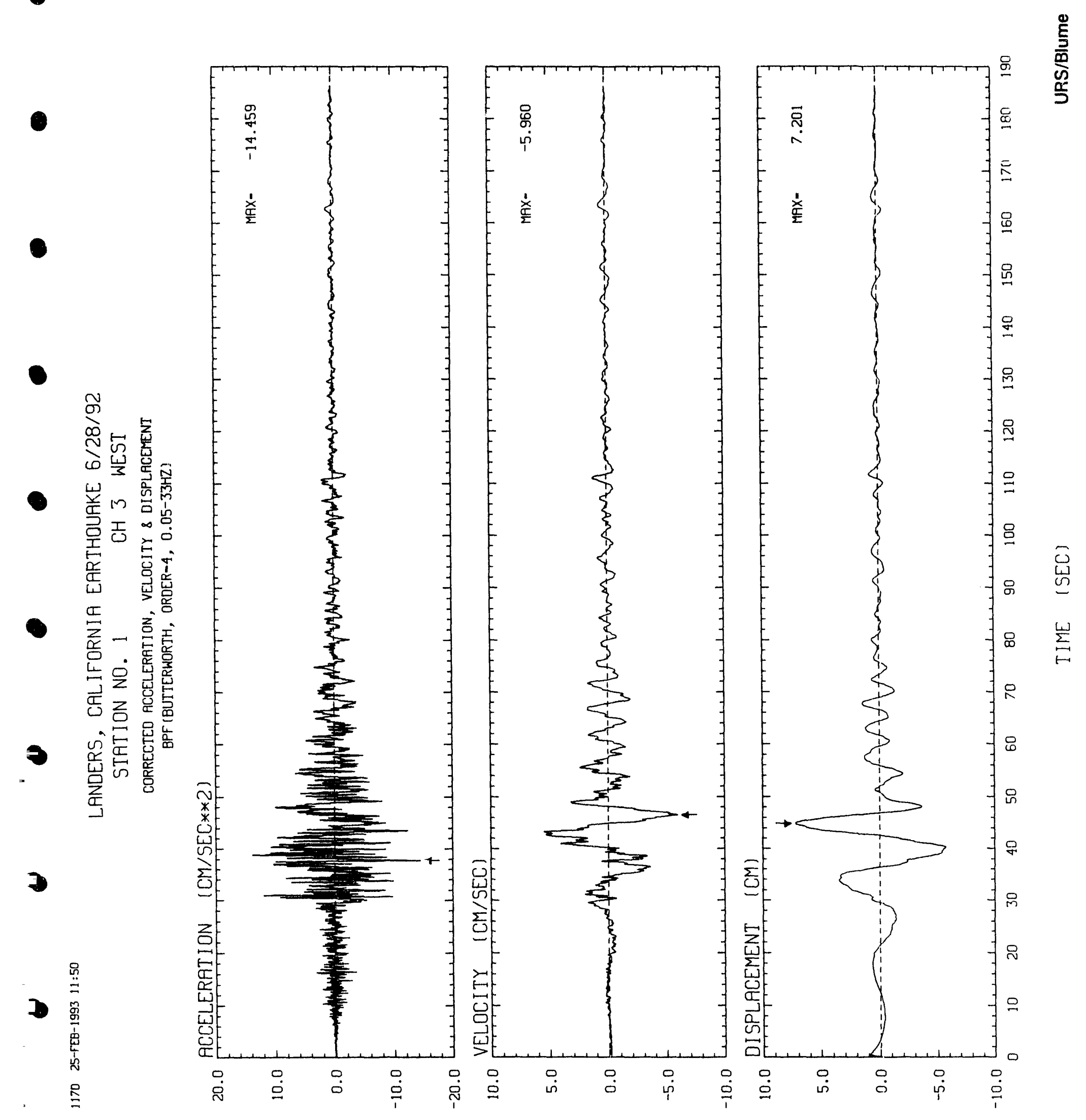



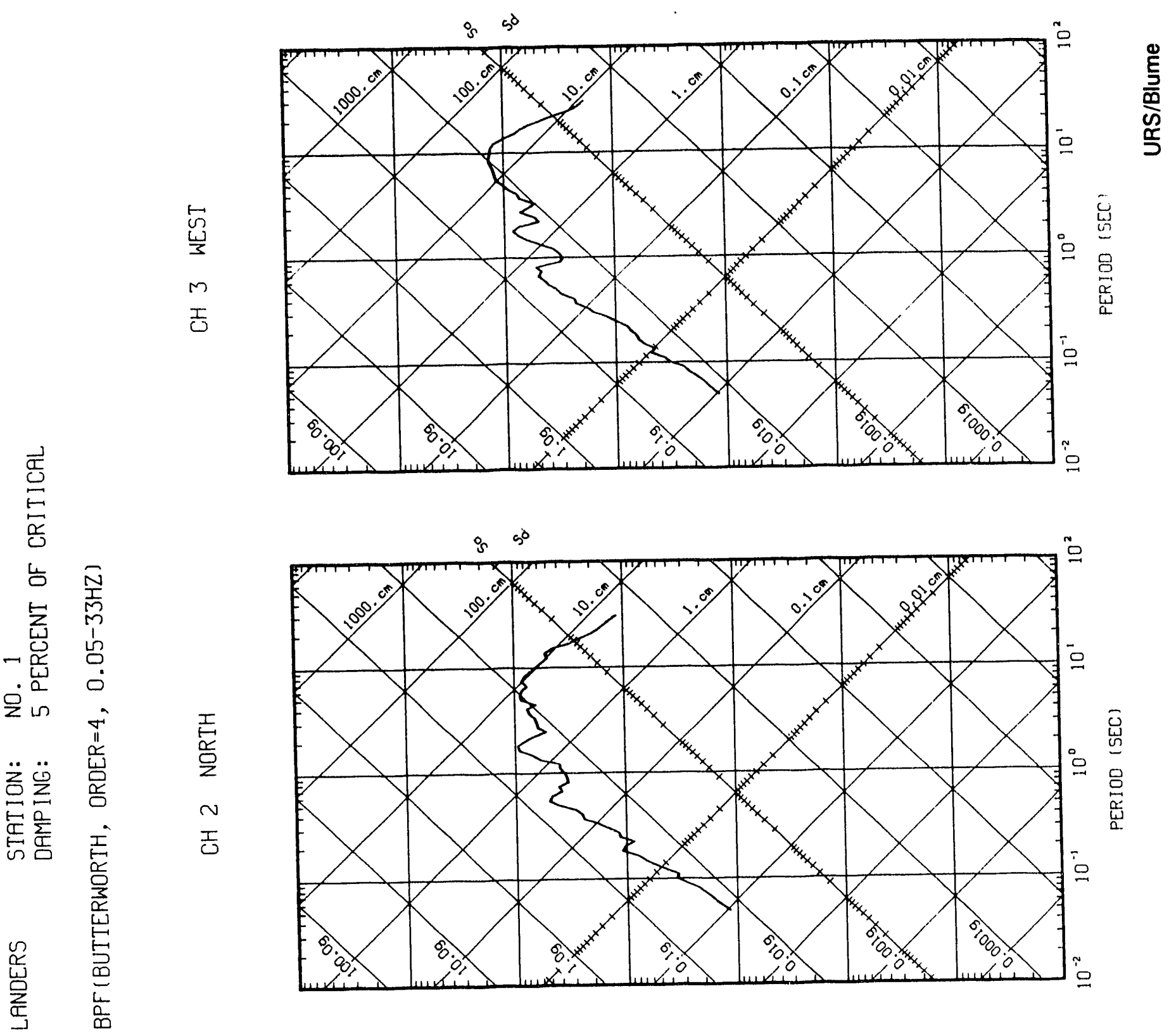

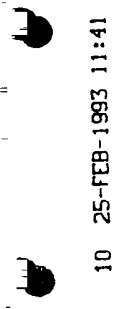

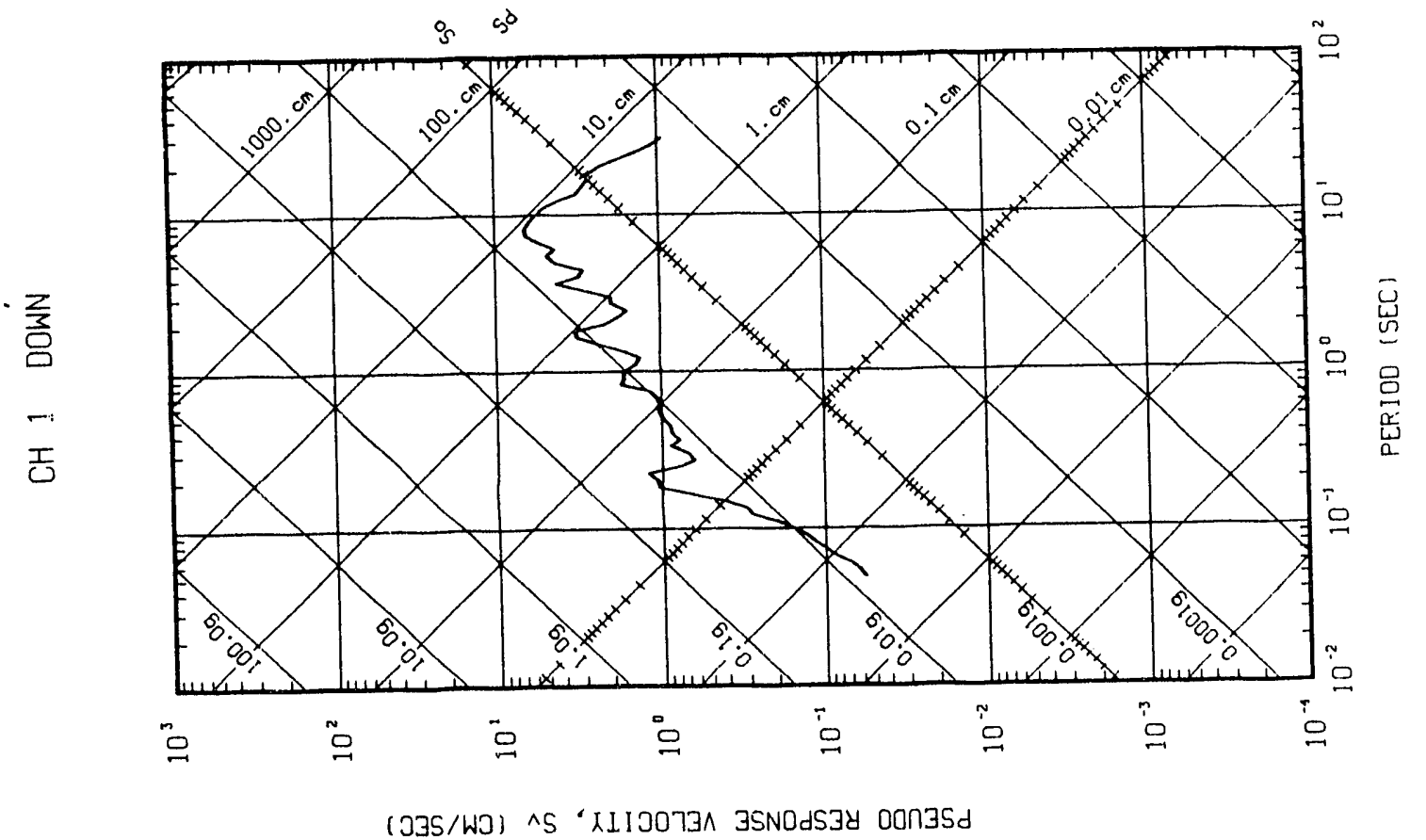


7

3

D

$b$ 


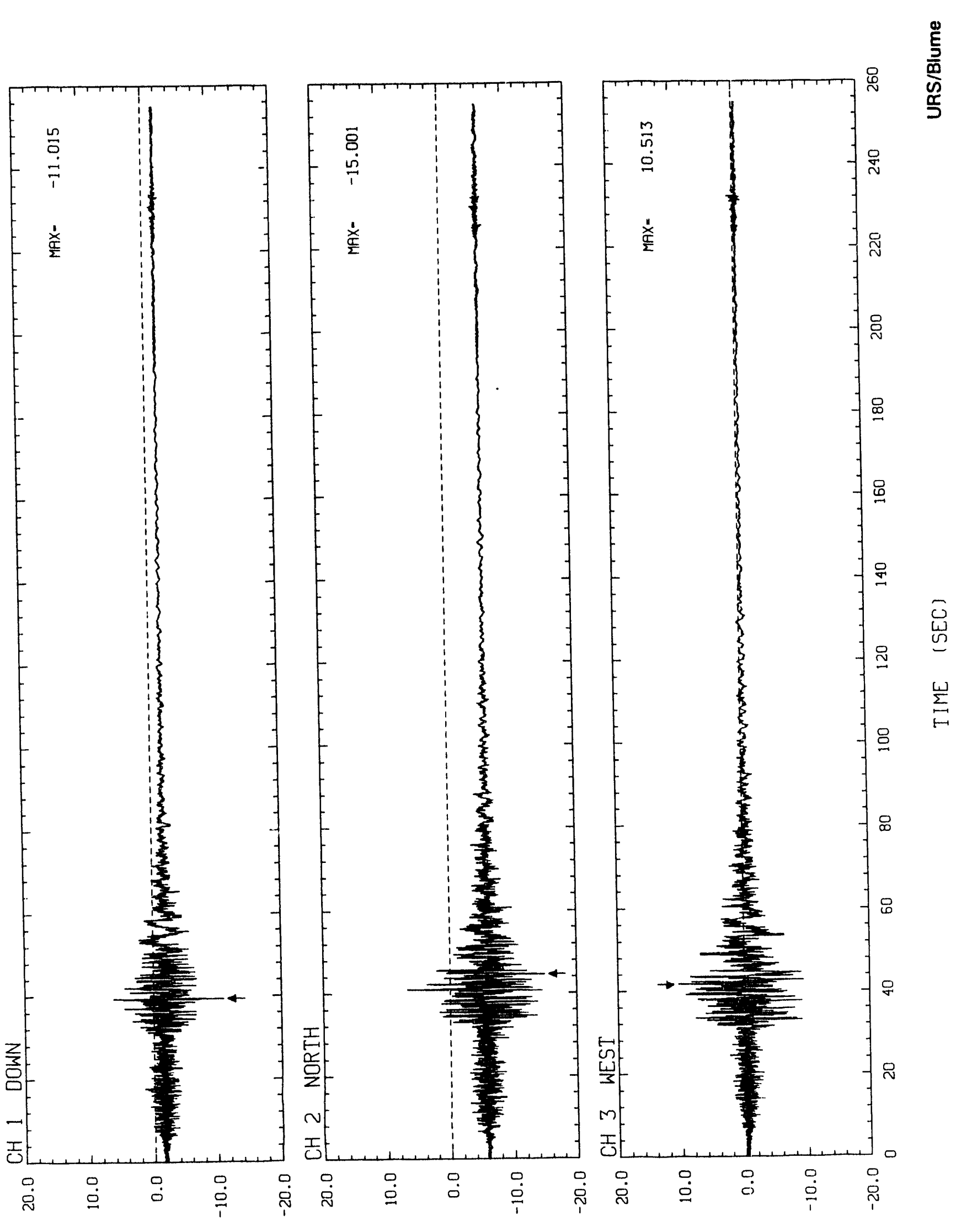

(2**03S/WJ) NOI I $4 y 37 \exists J 3 \forall$ 

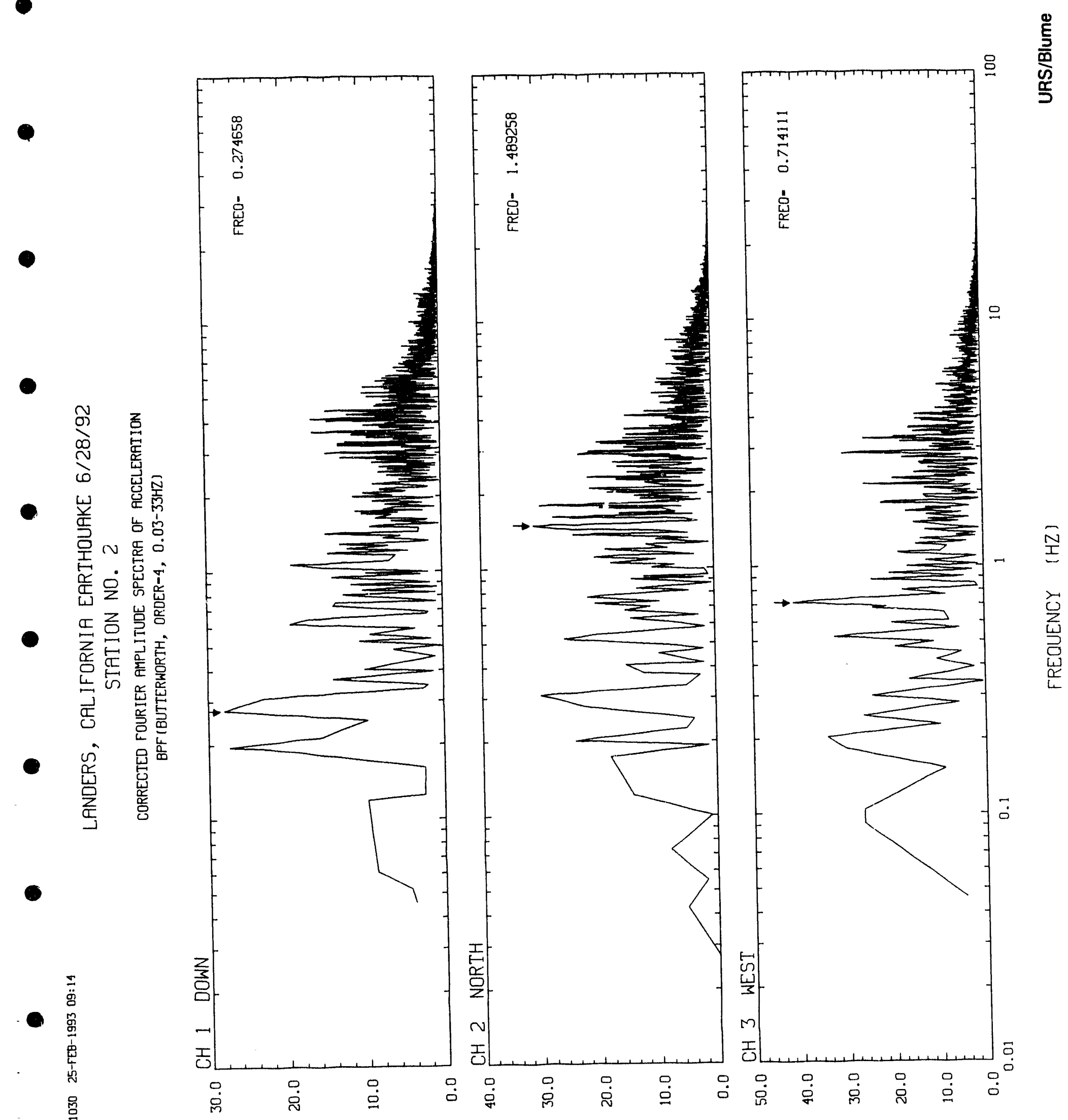

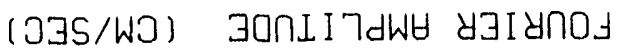




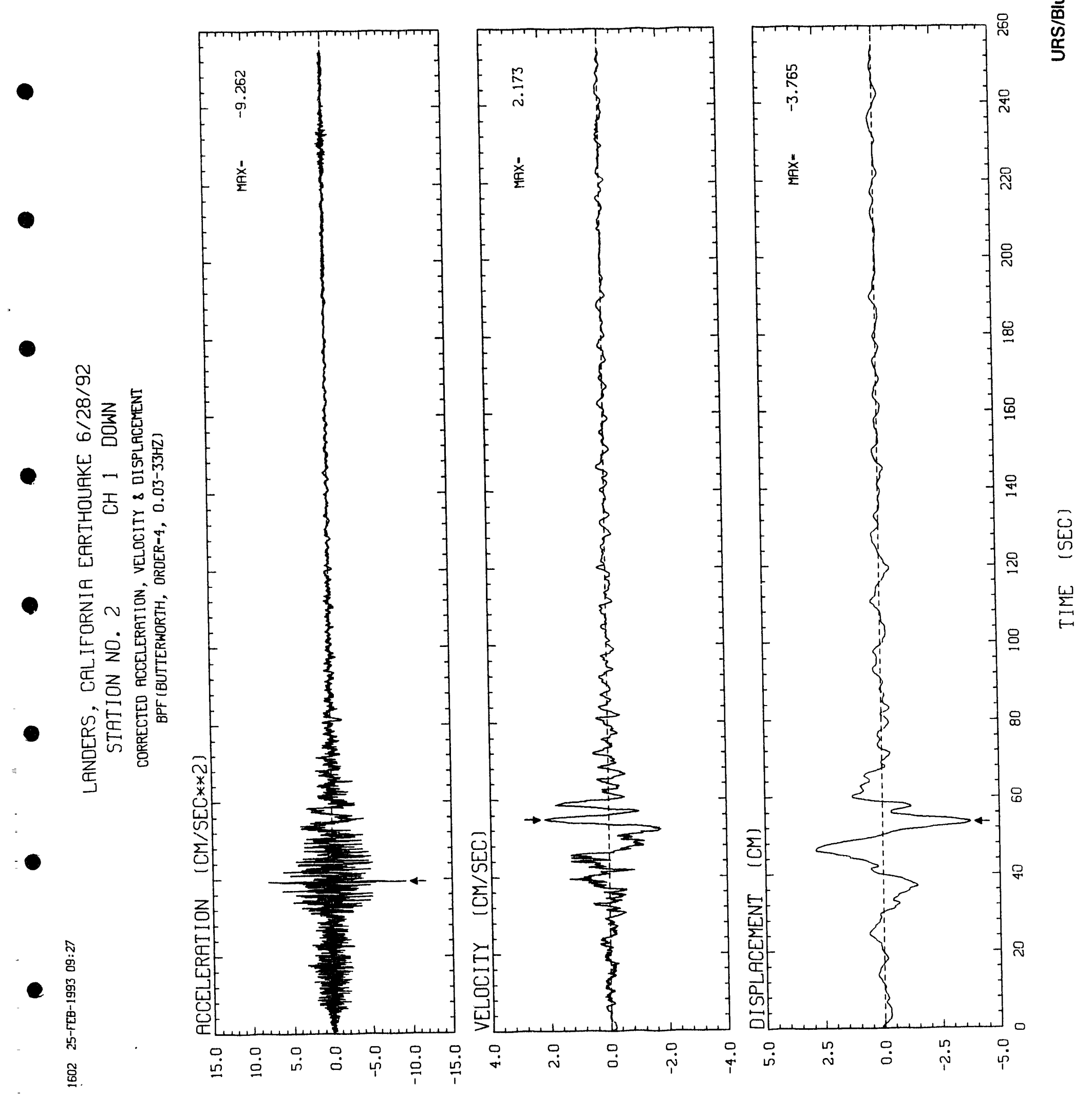


- 


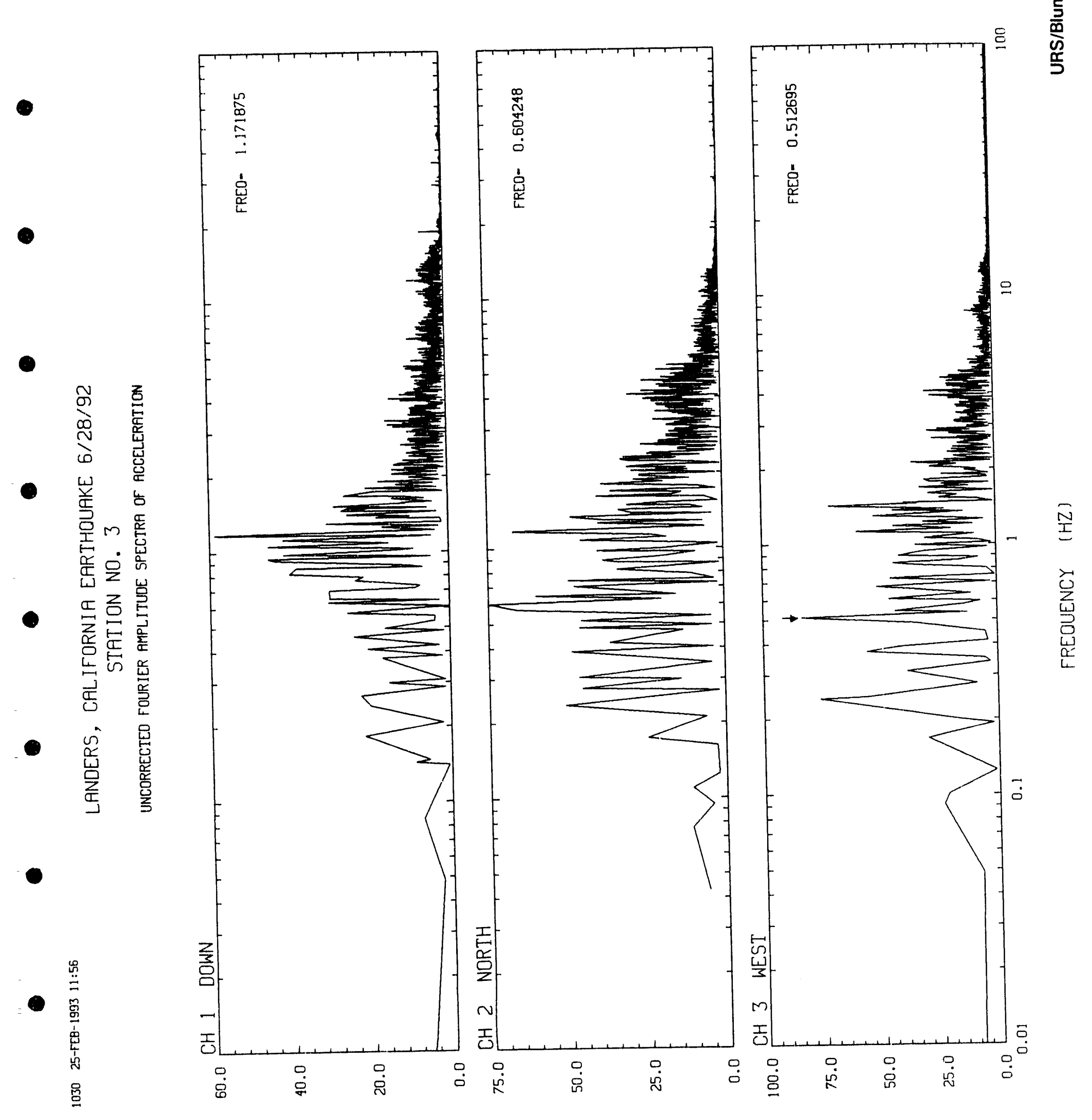

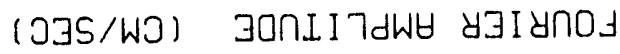




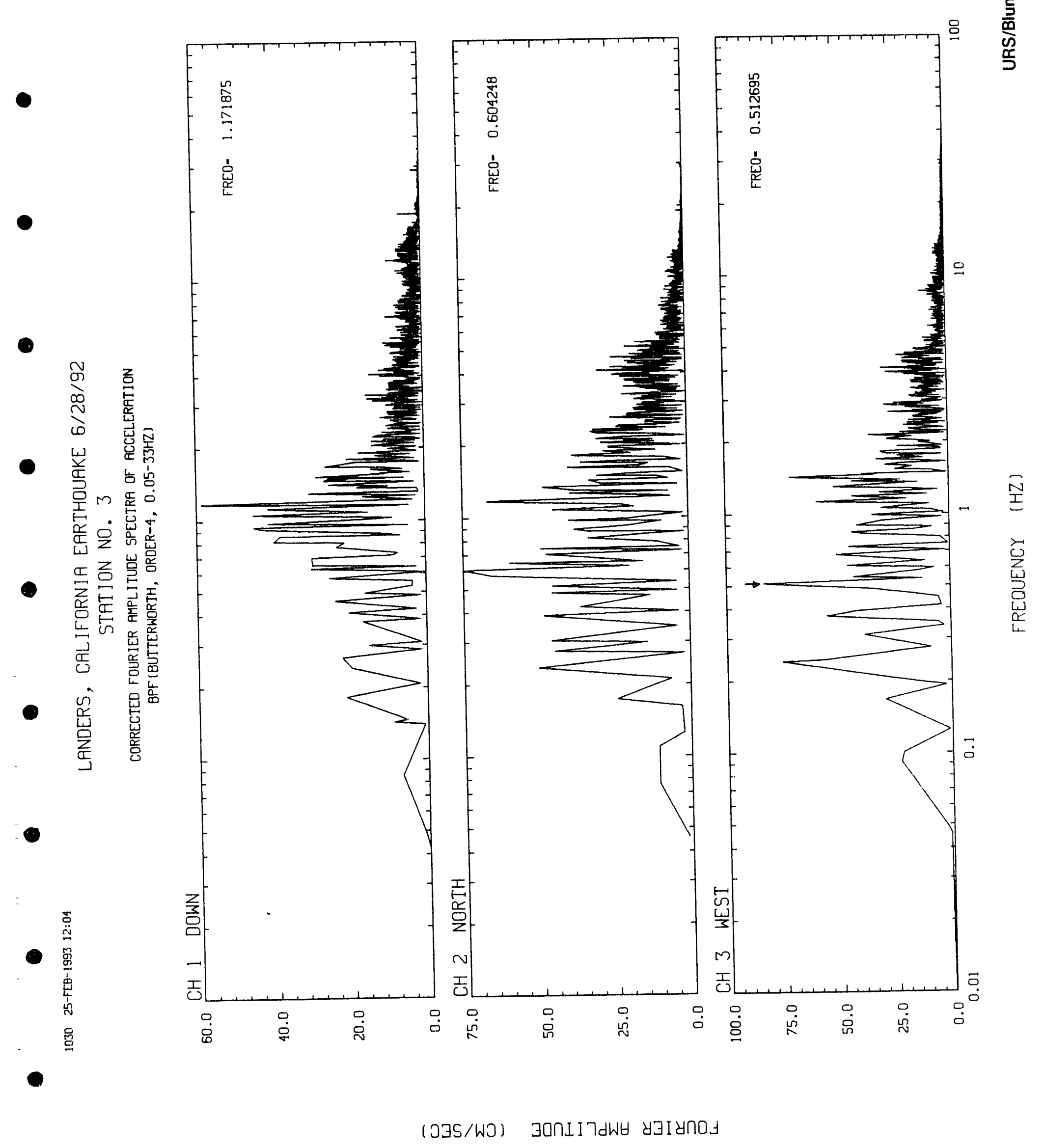




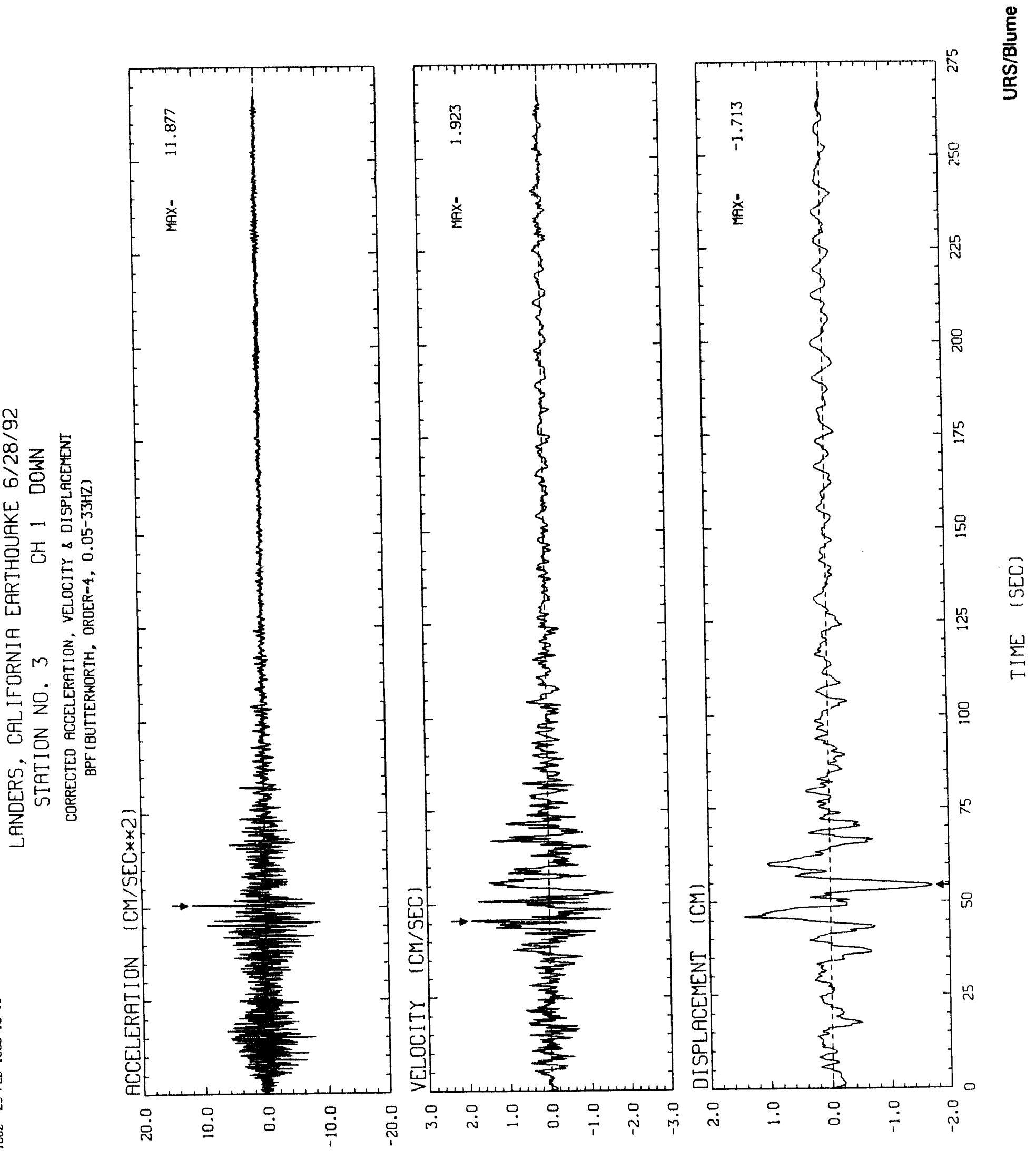

30กI I 7dWH 


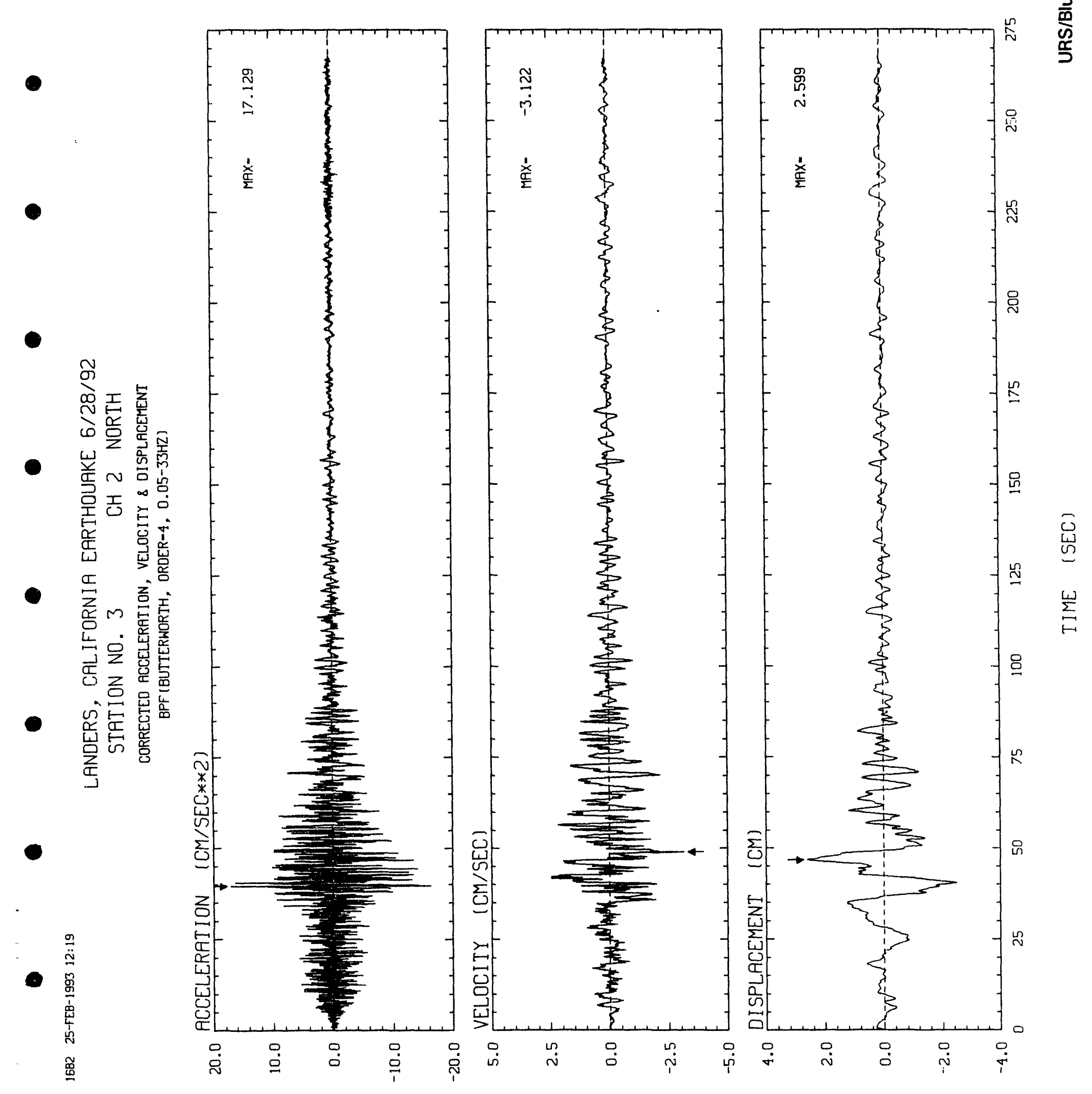




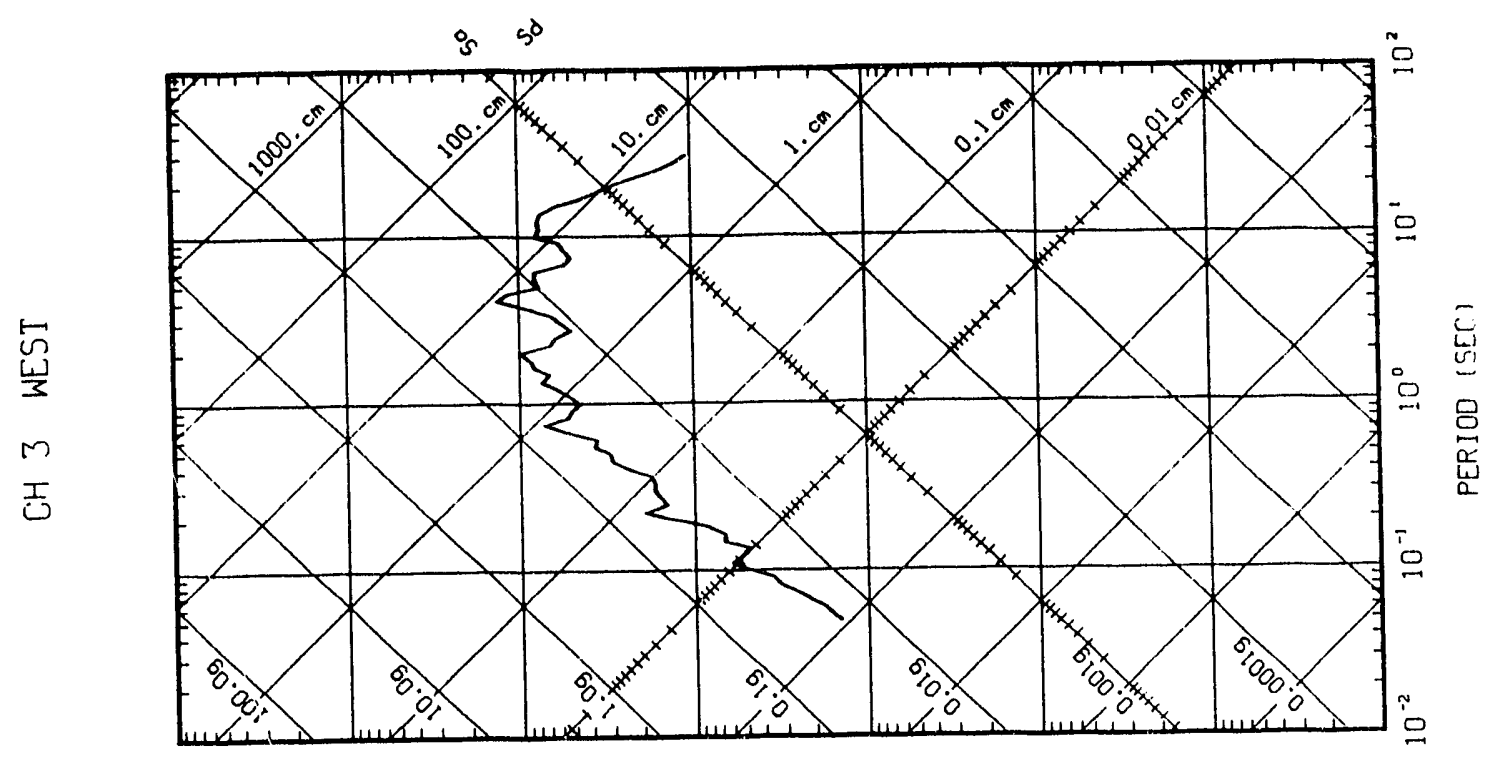

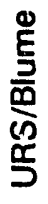
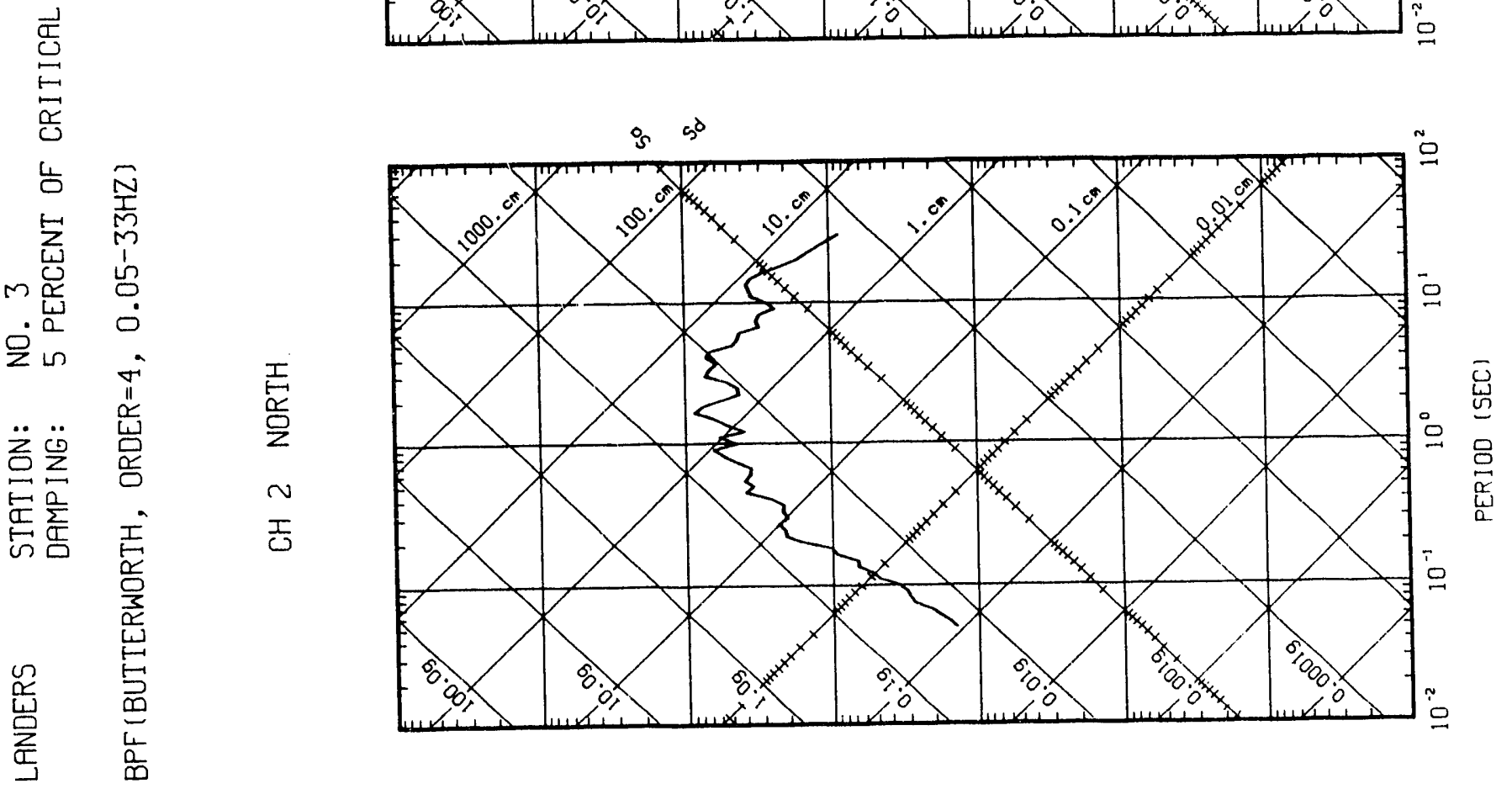

点岕悹

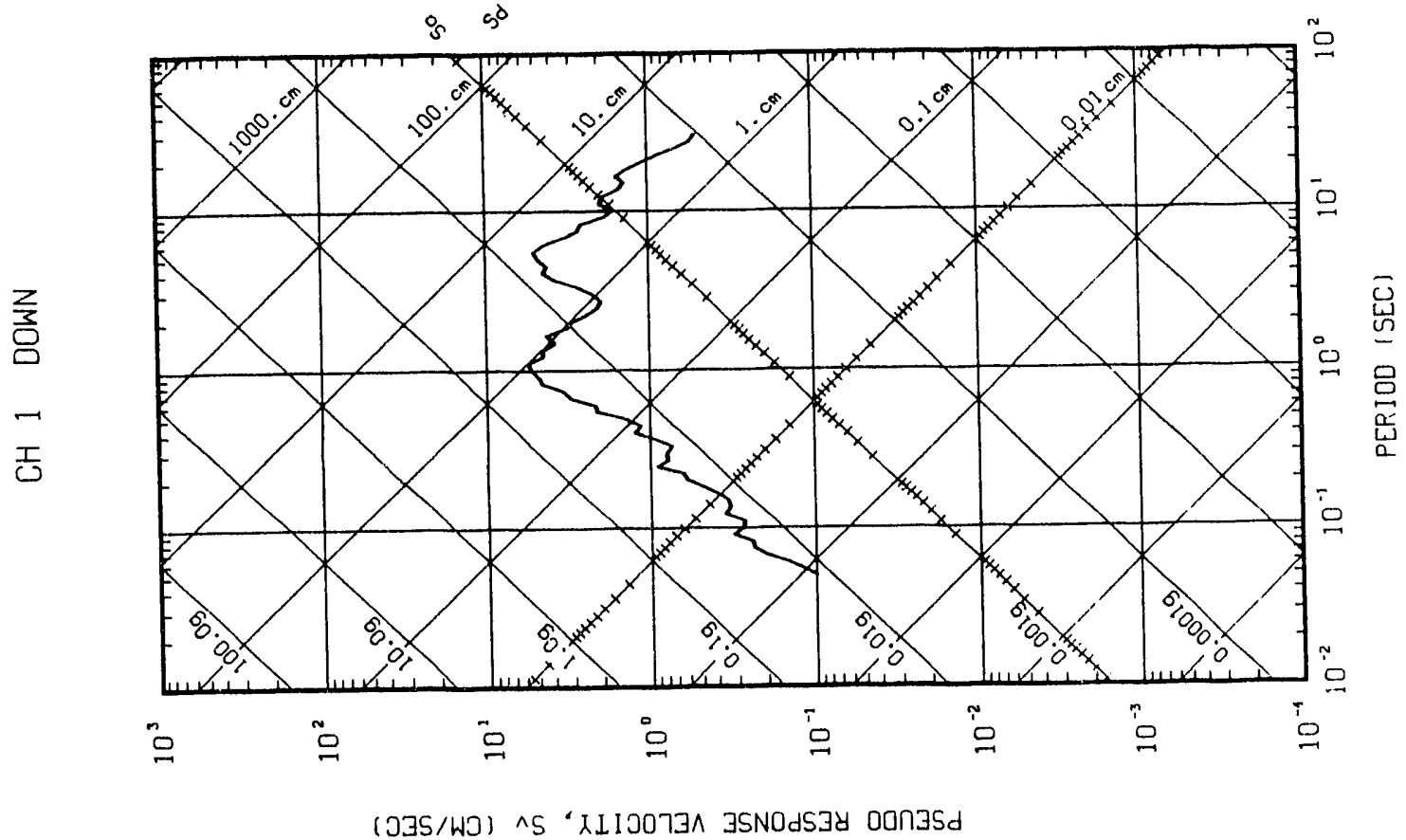




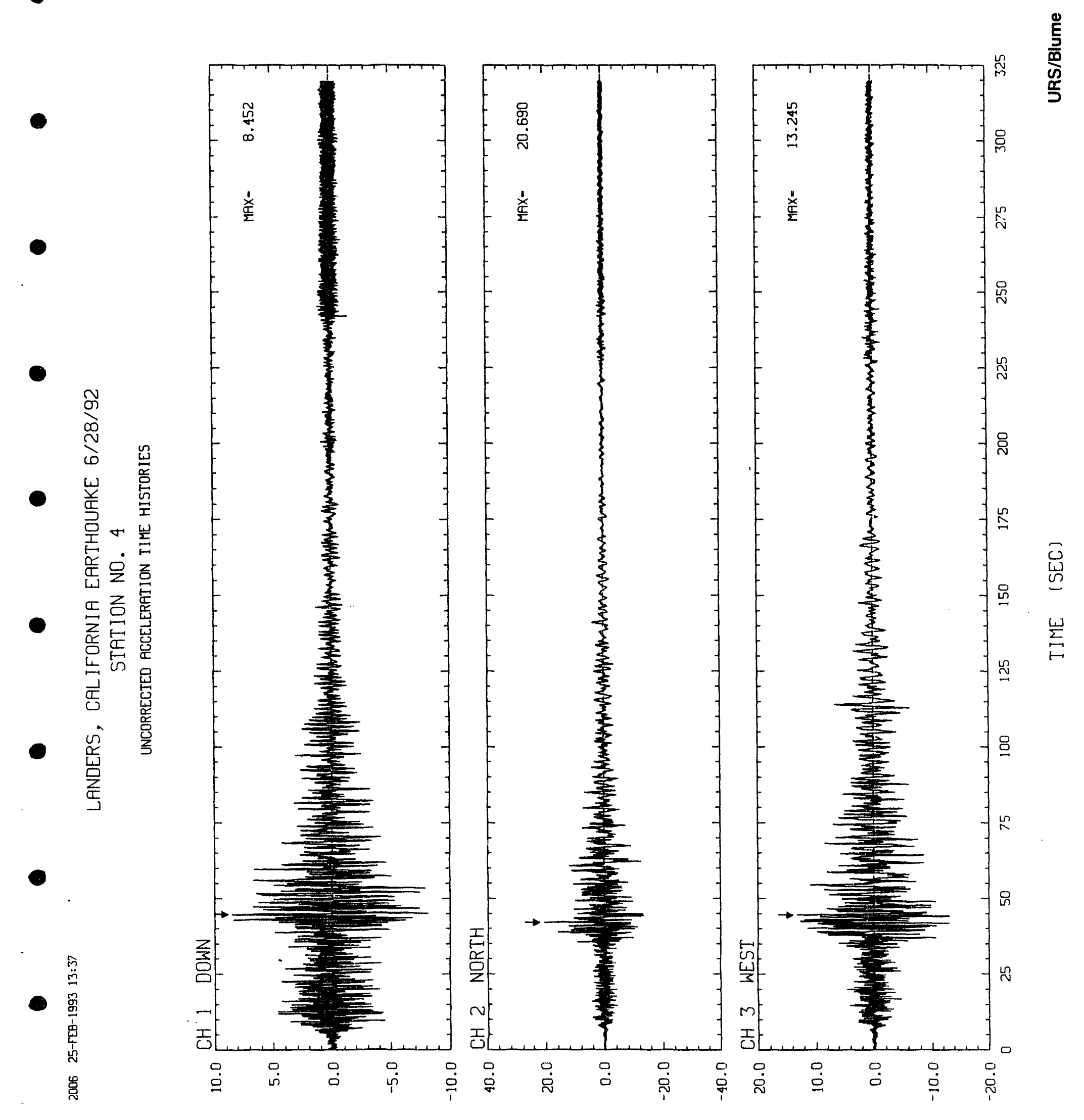

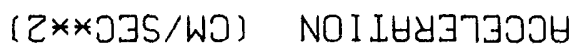




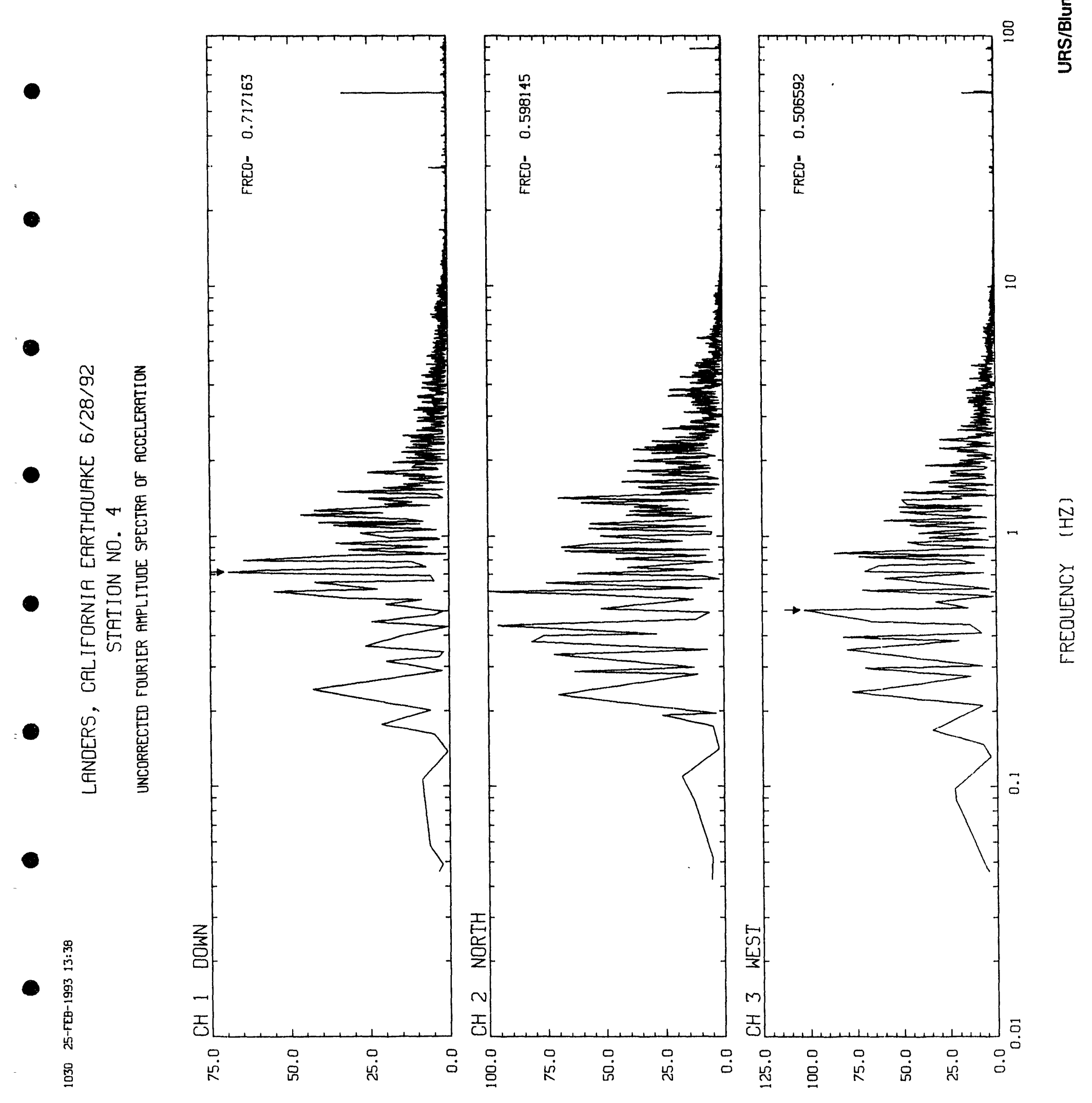

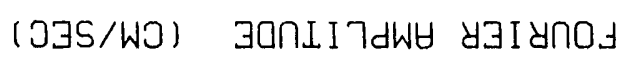




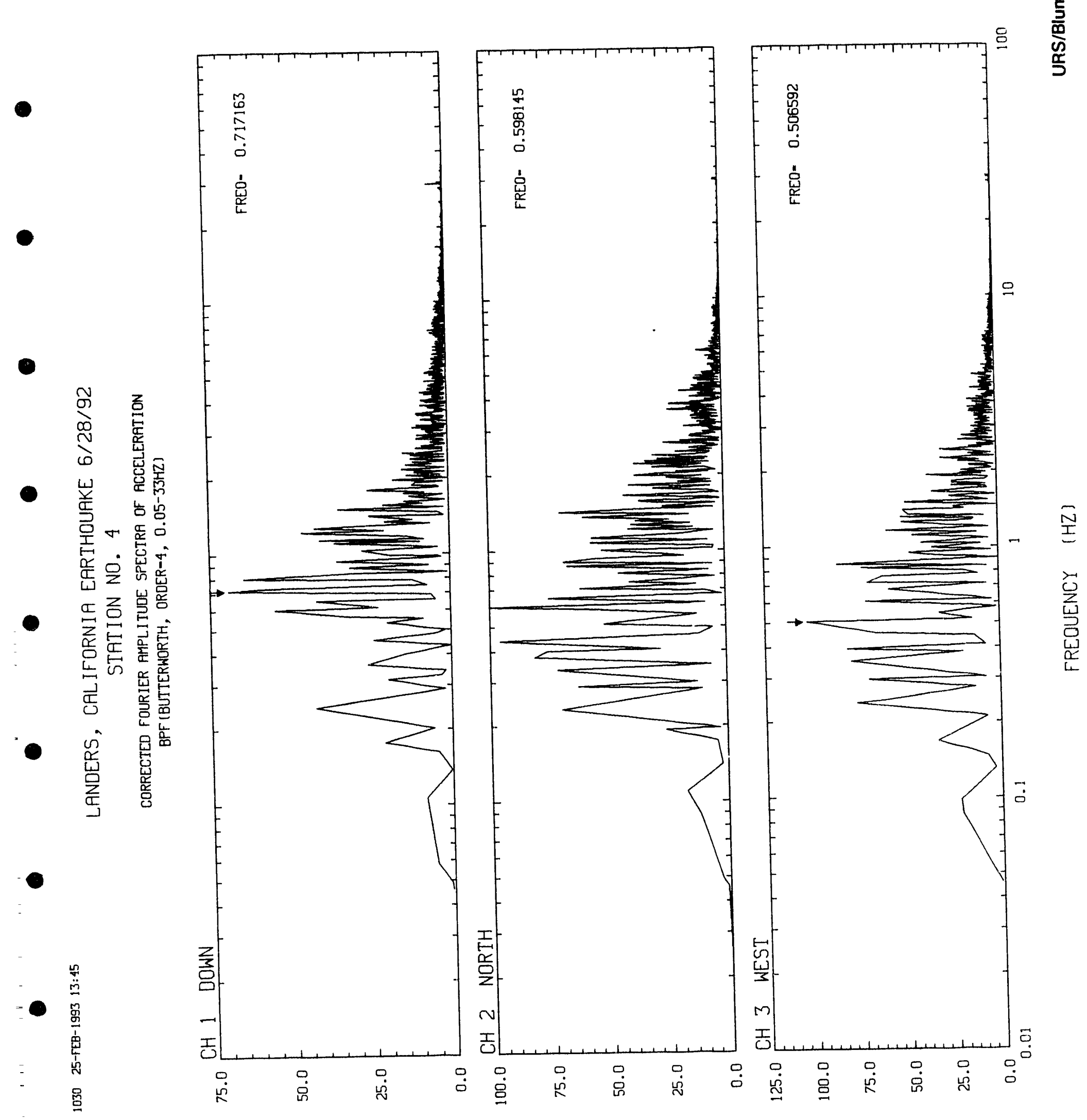

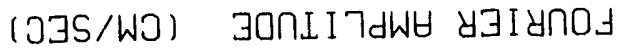



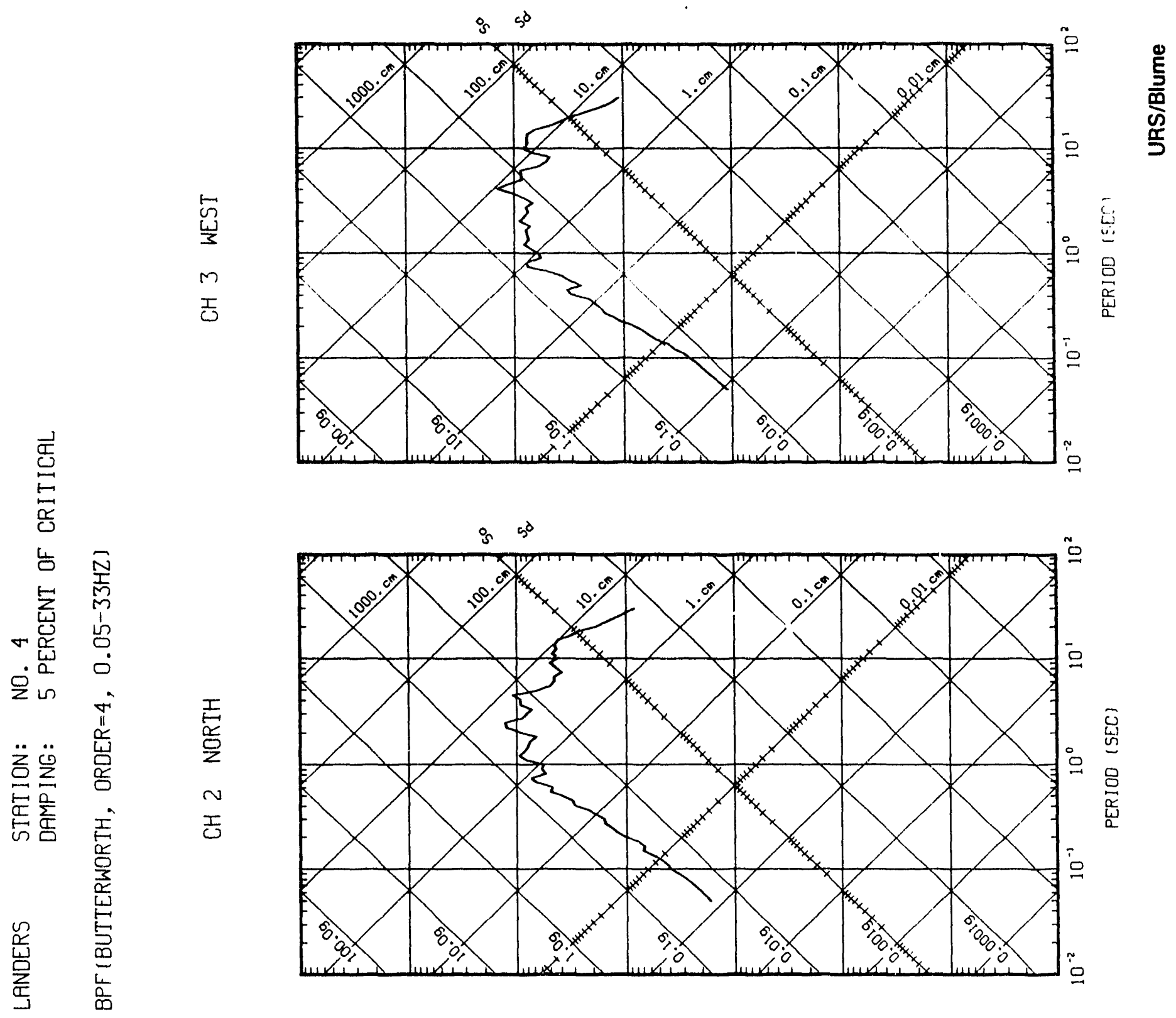

苍岕

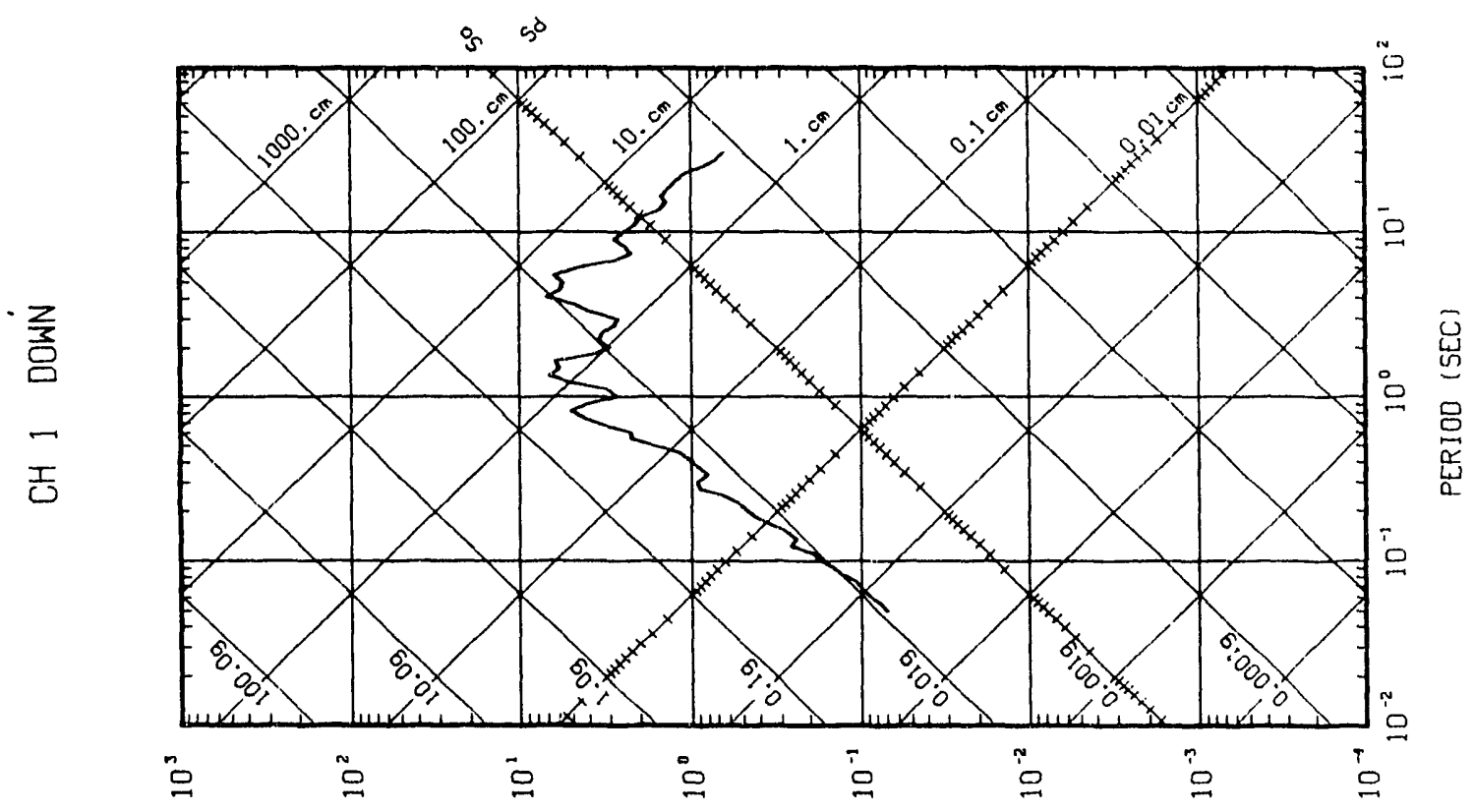

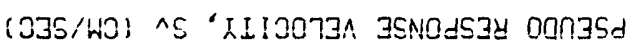


$\bullet$

-

-

-

-

-

1)

Z

Z

-

○

-

- 


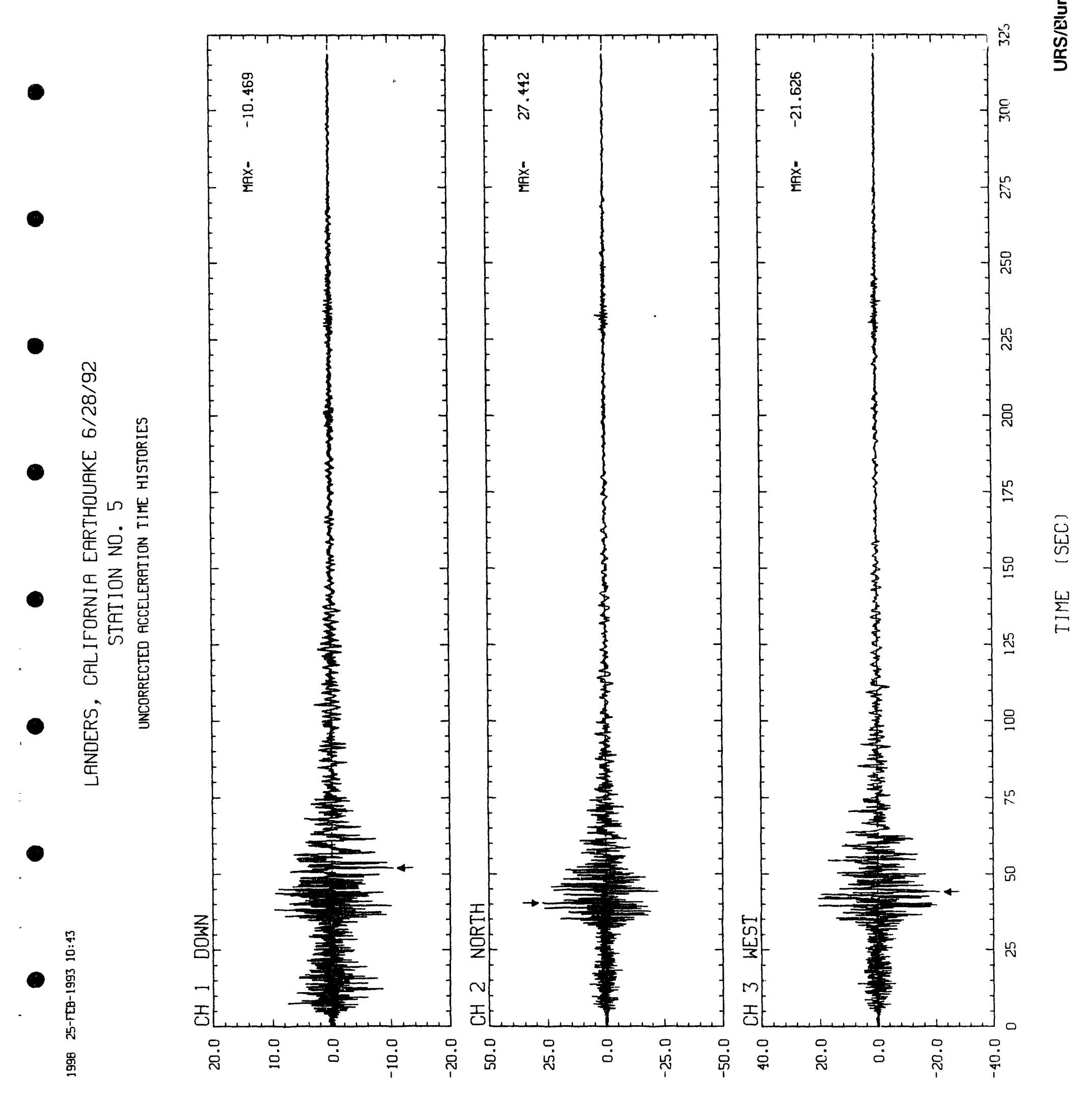

(2**3.35/WJ) NOI I $4 y 37 \exists J 3 \forall$ 


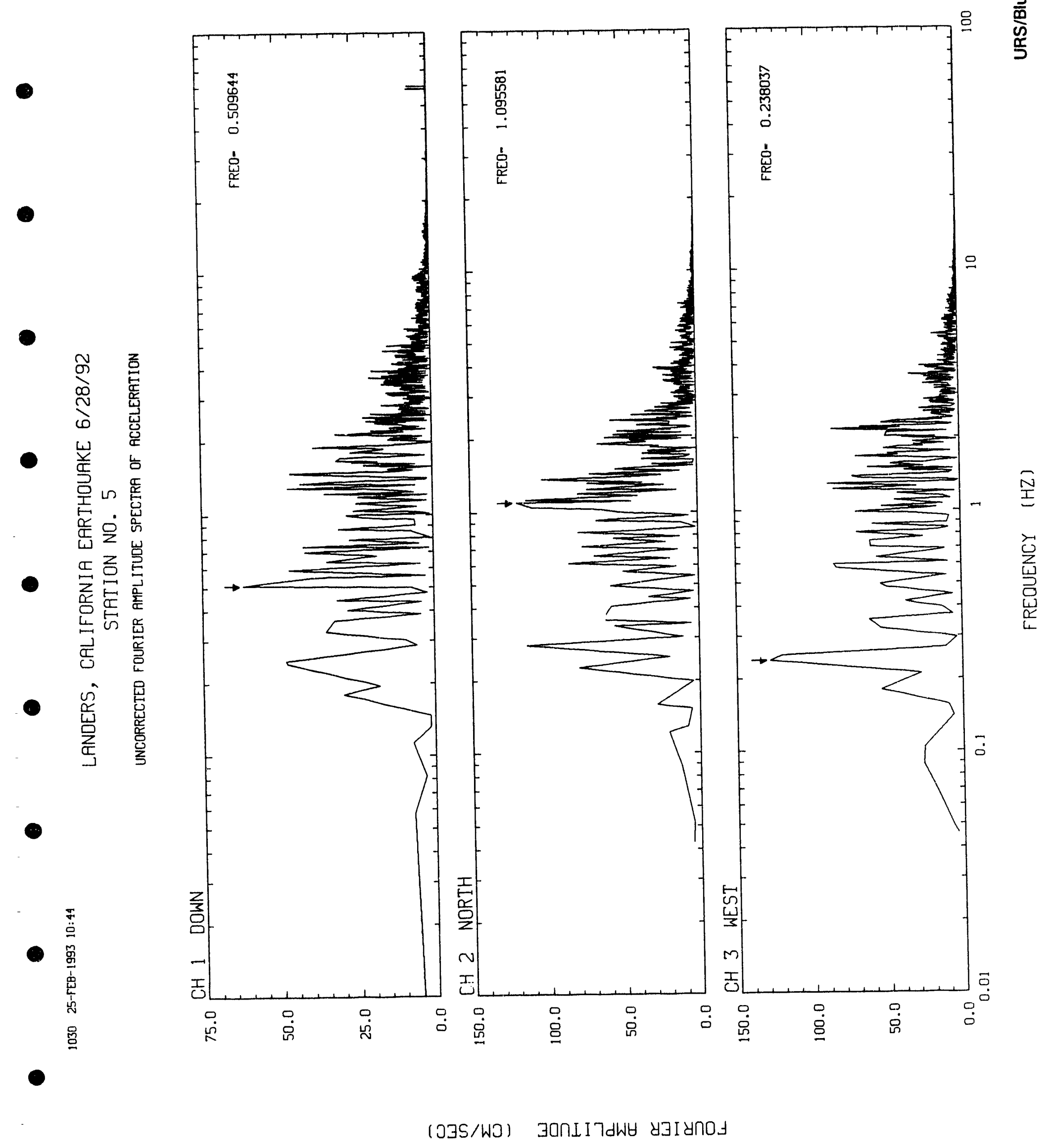



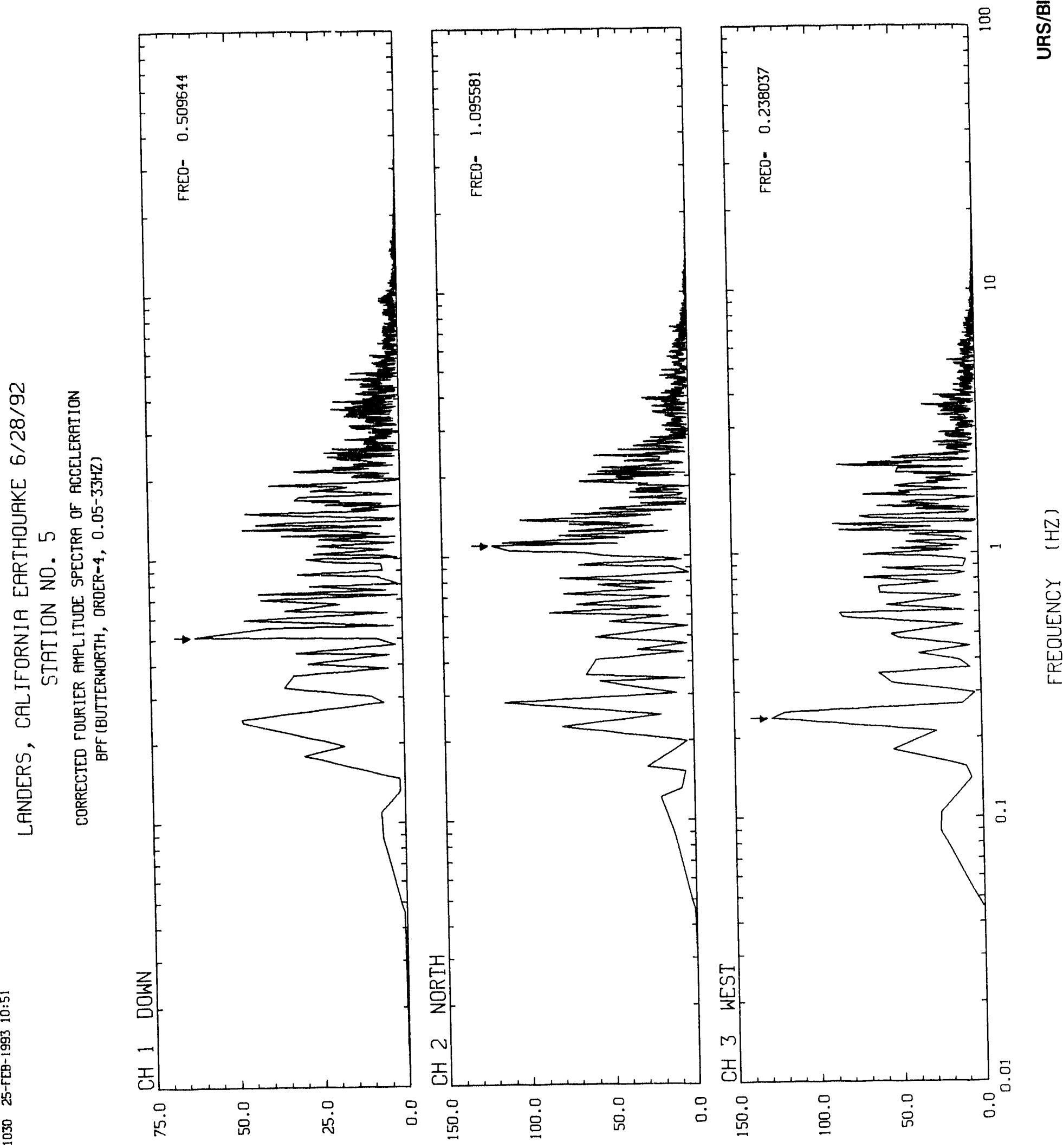

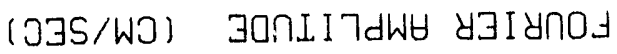




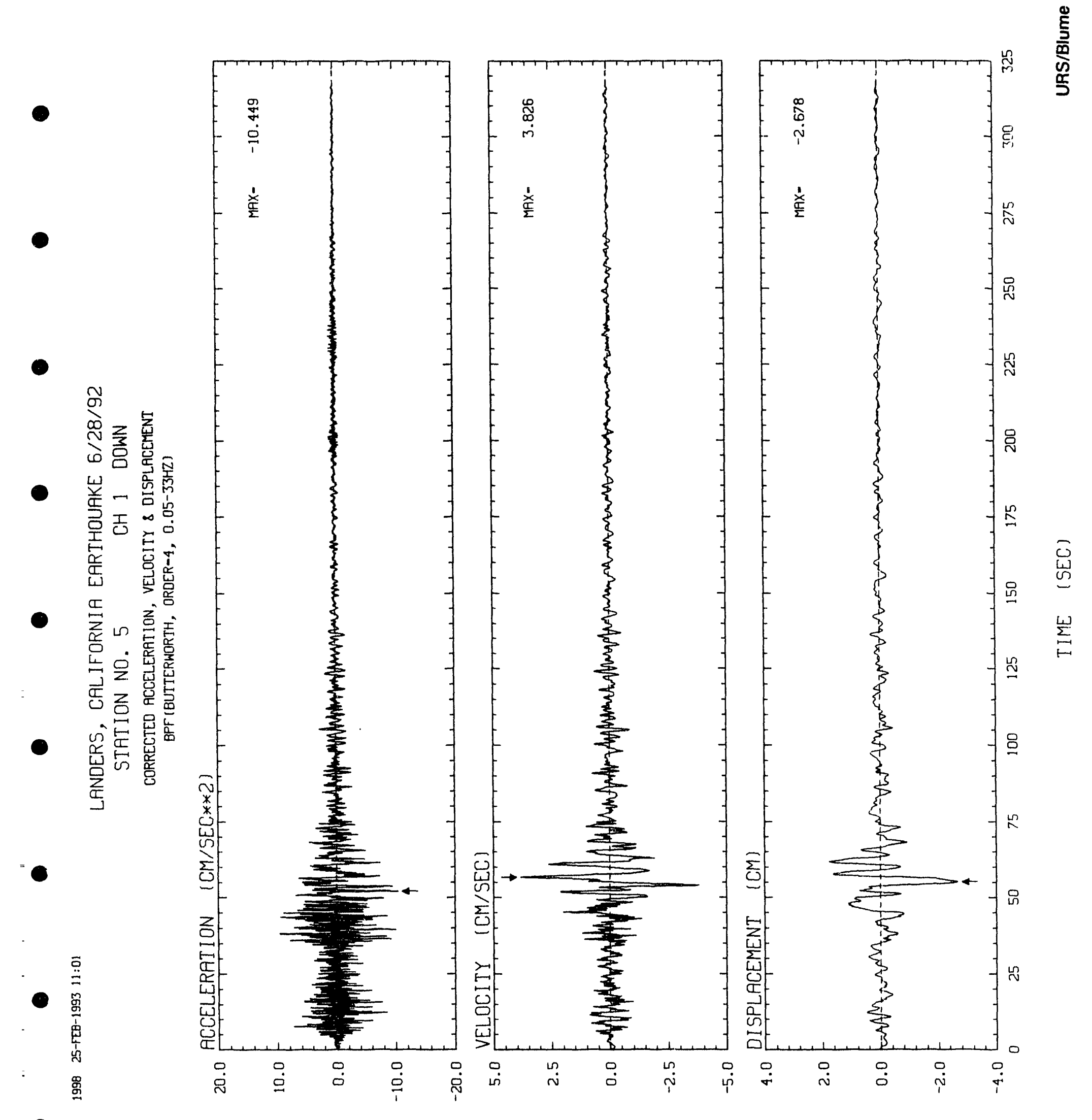




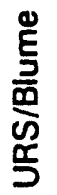
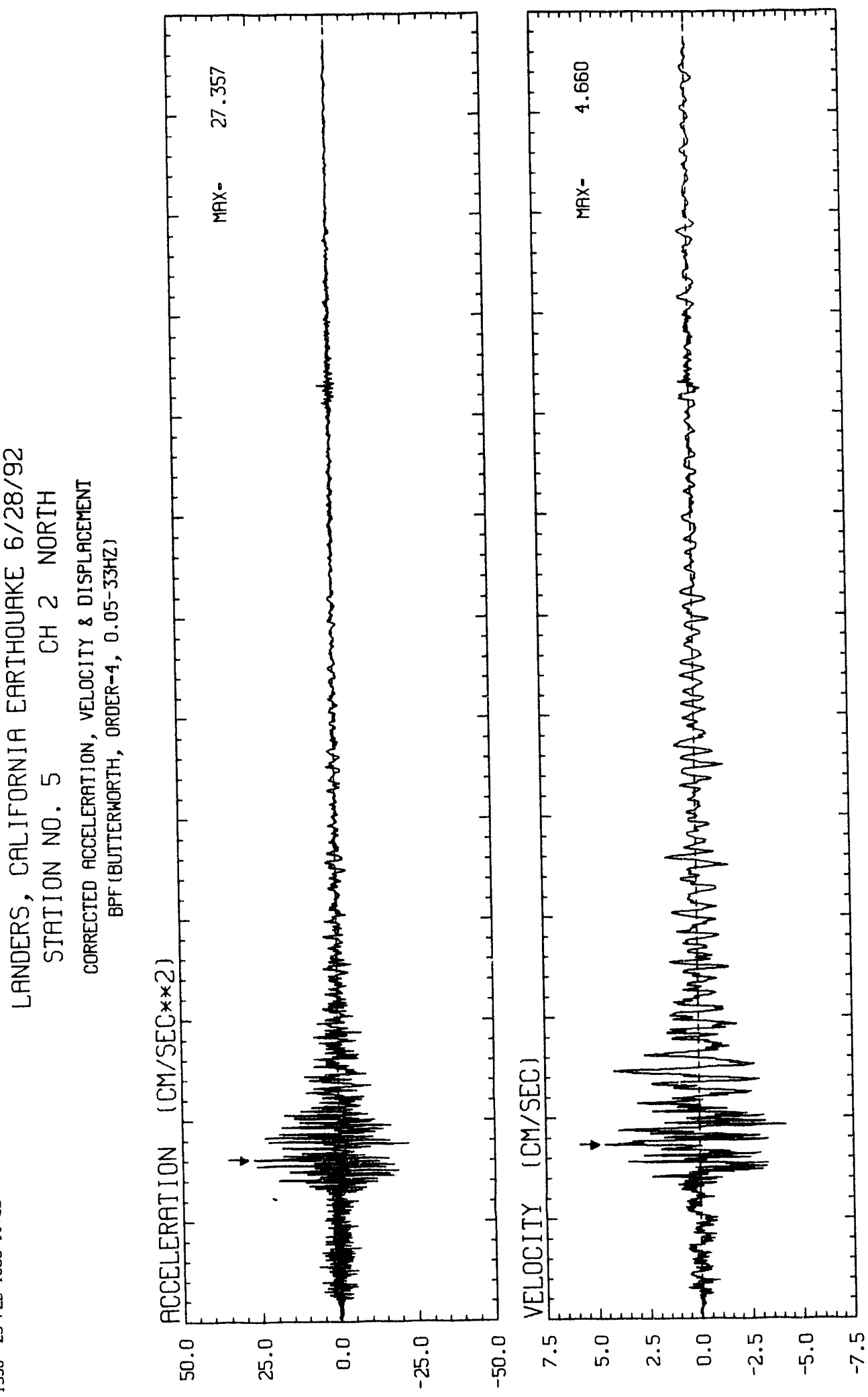

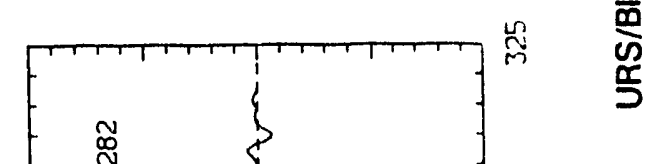

范

齐

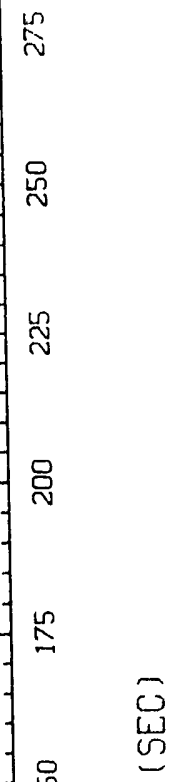

-

$\bullet$

$\bullet$

崖 $\sim$ 总产 문ㄷㄴ잉

䍃 岂咅

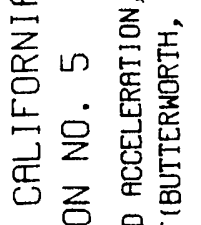

的总总

䍃焉㟧

.

惫

m

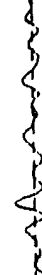
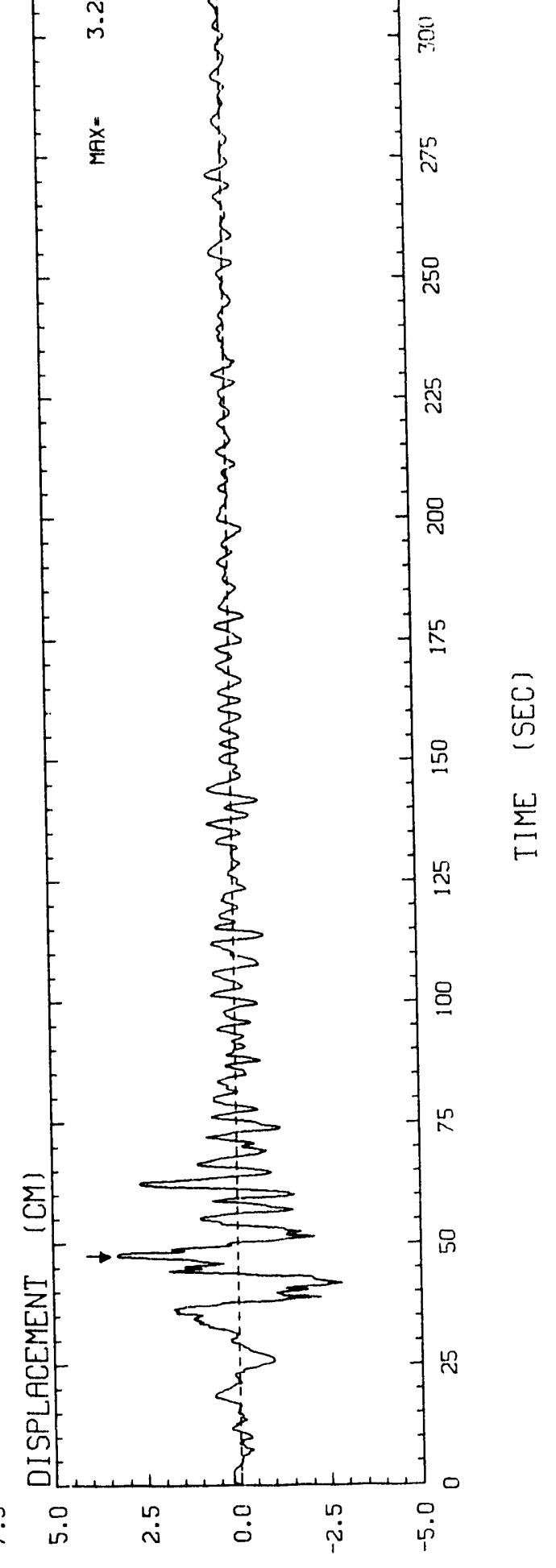


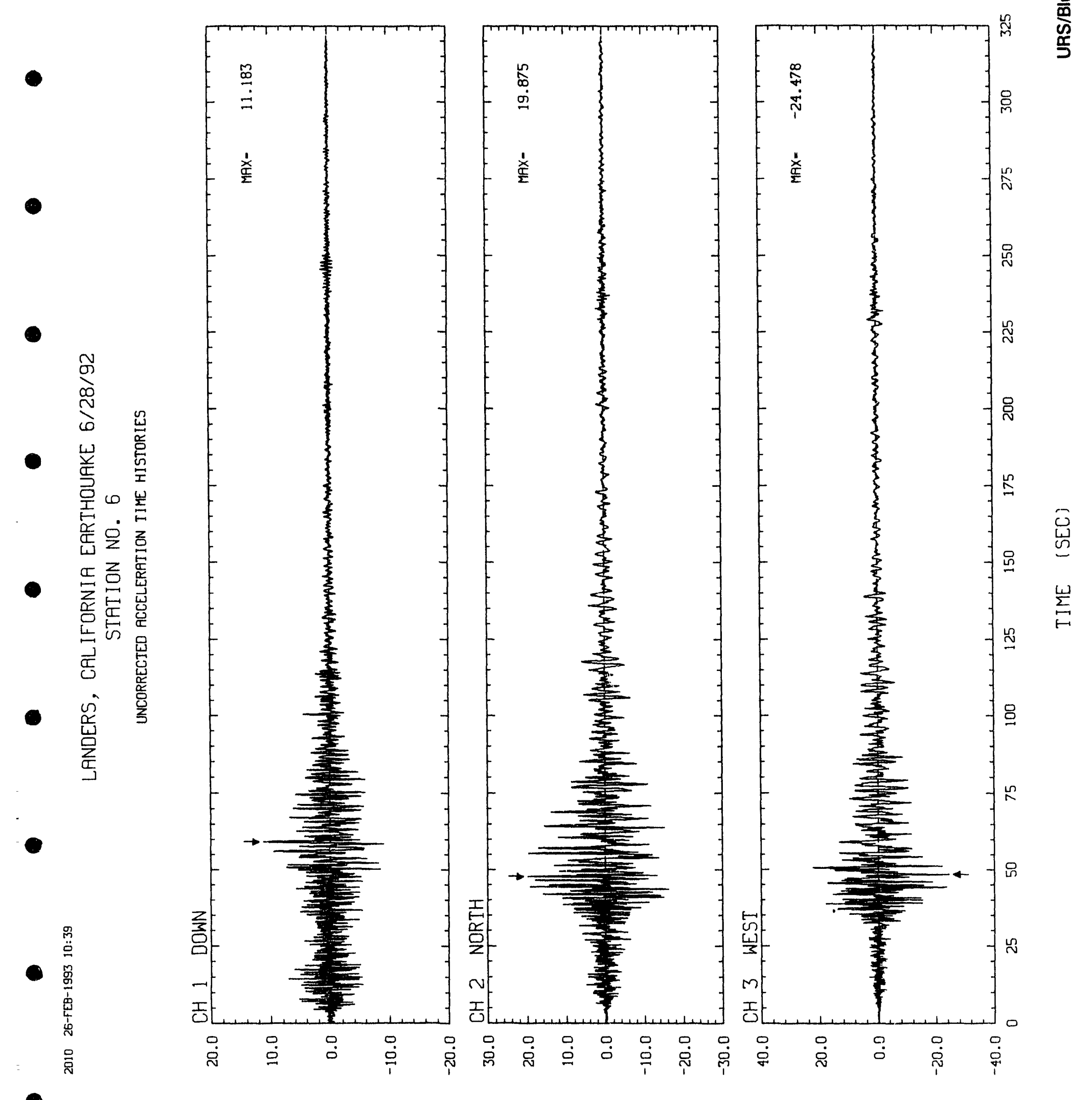

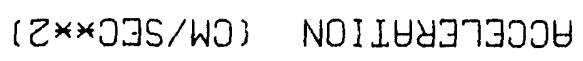




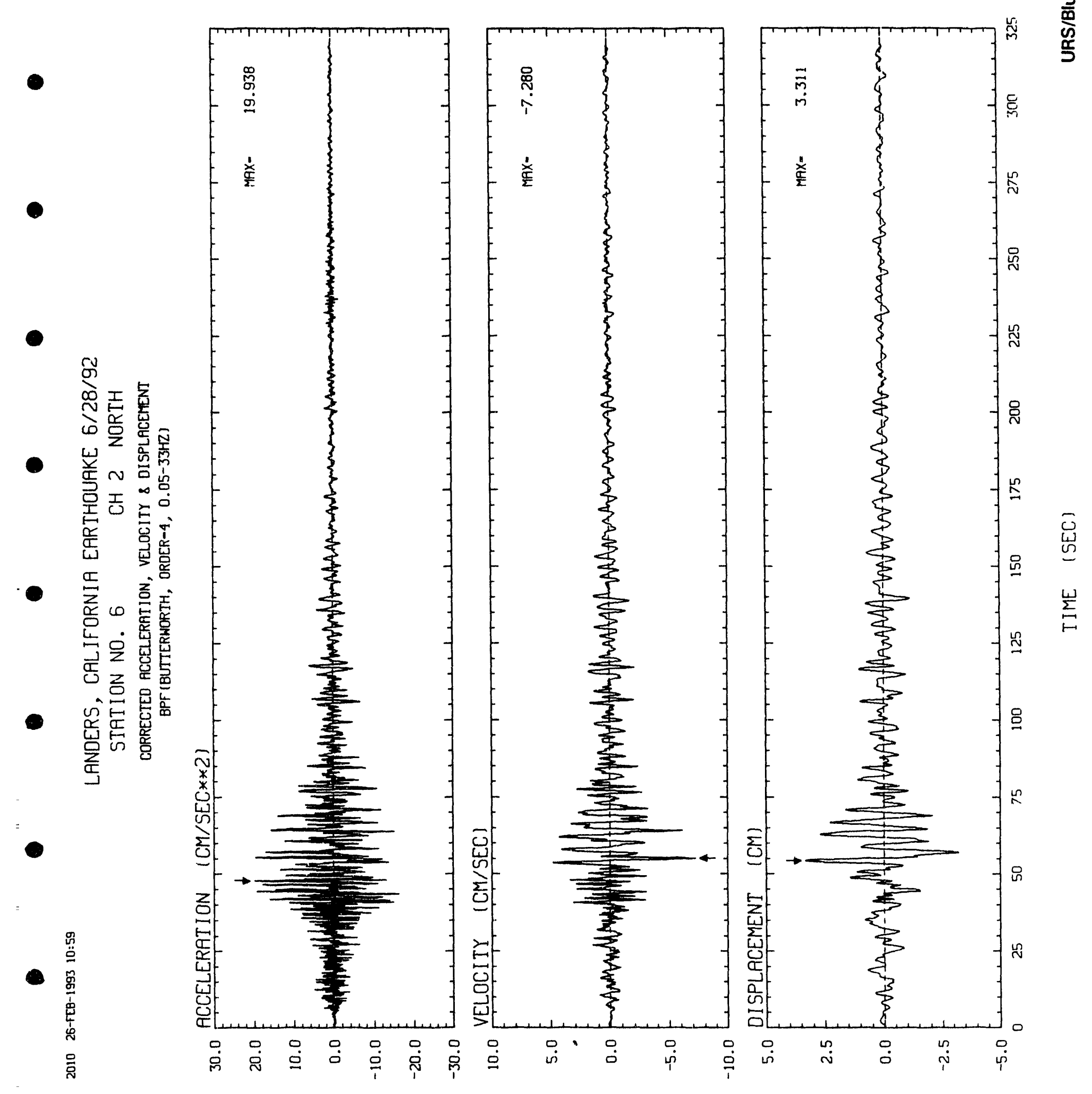

JOก I I 7dWH 

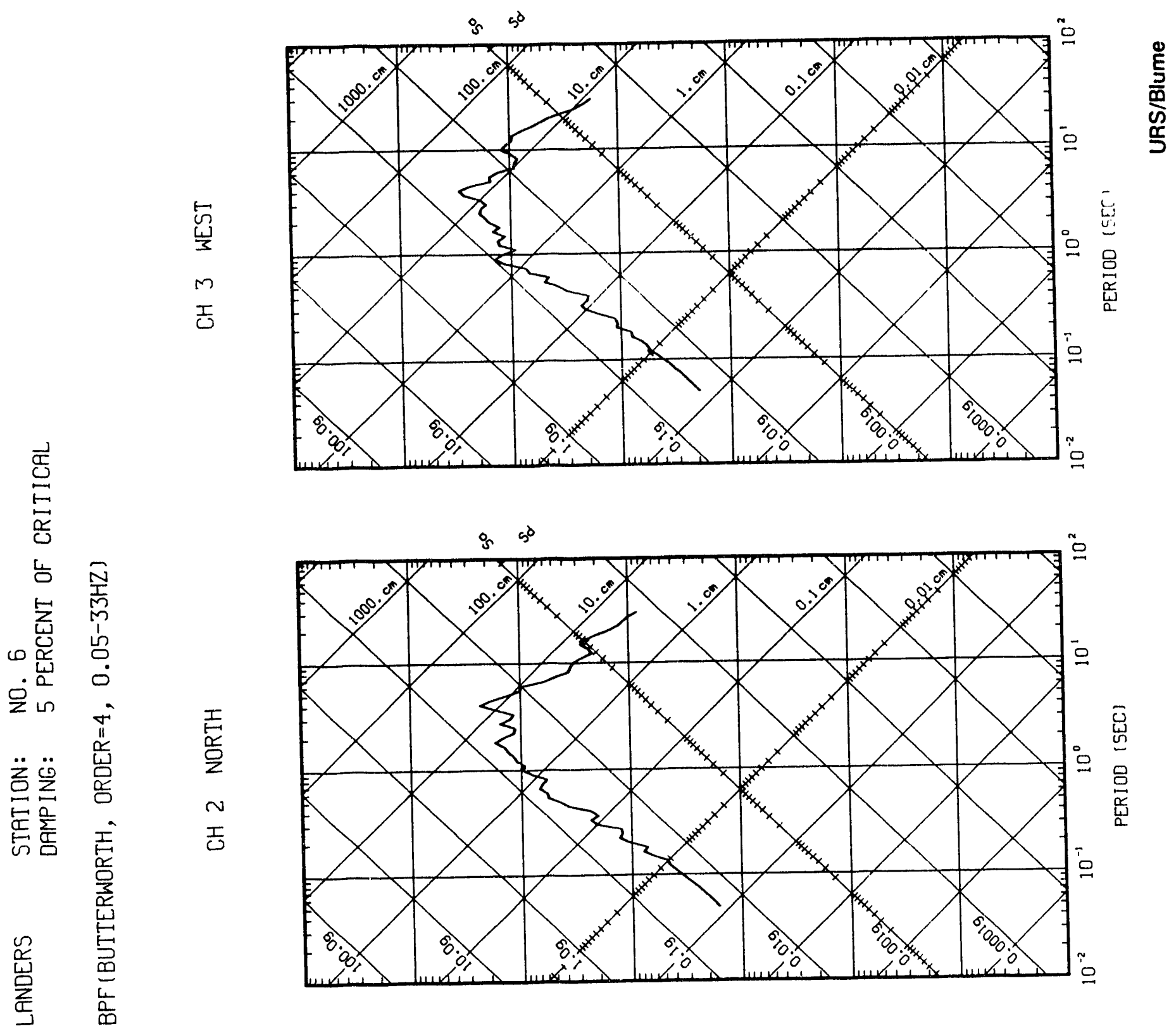

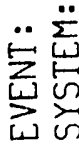

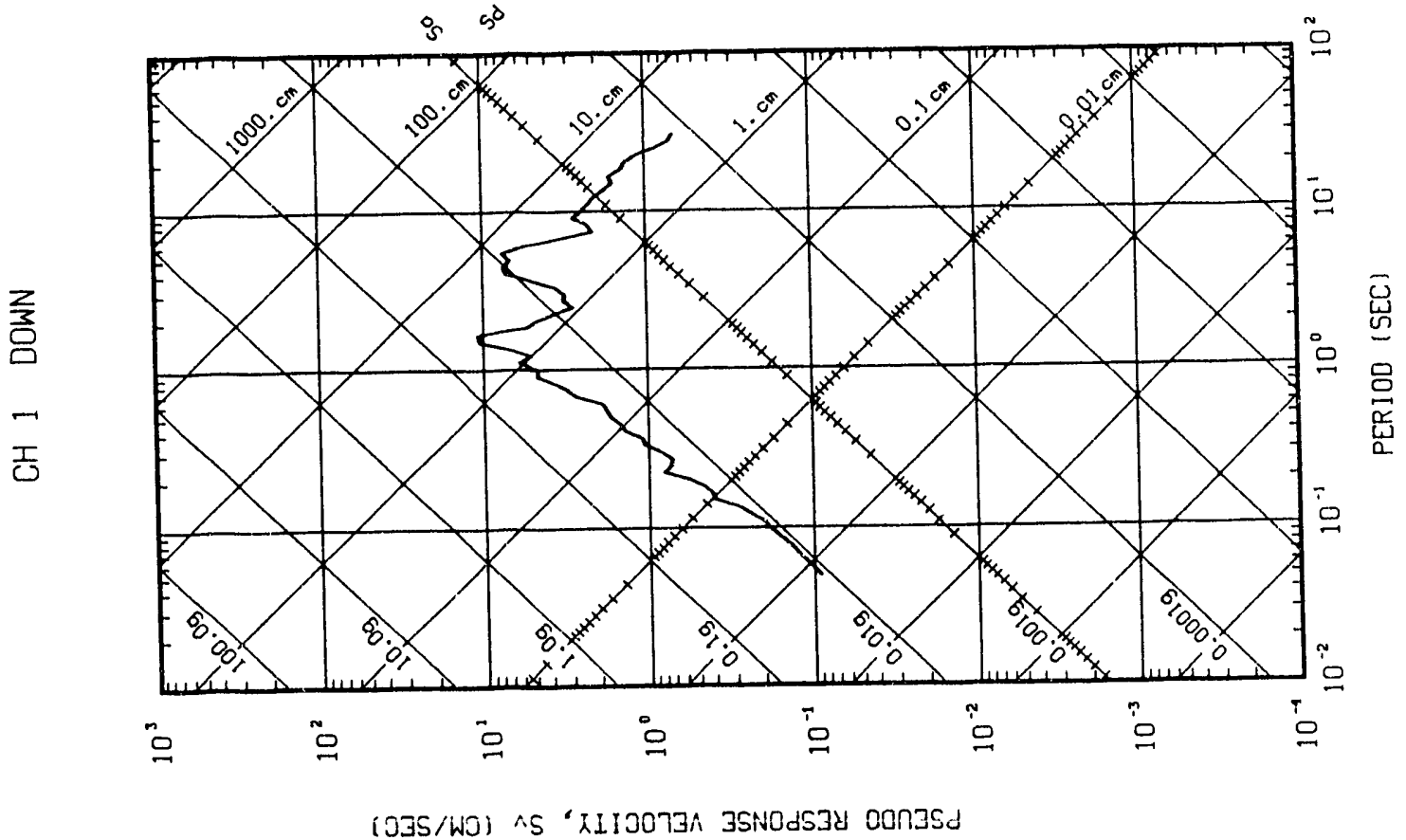




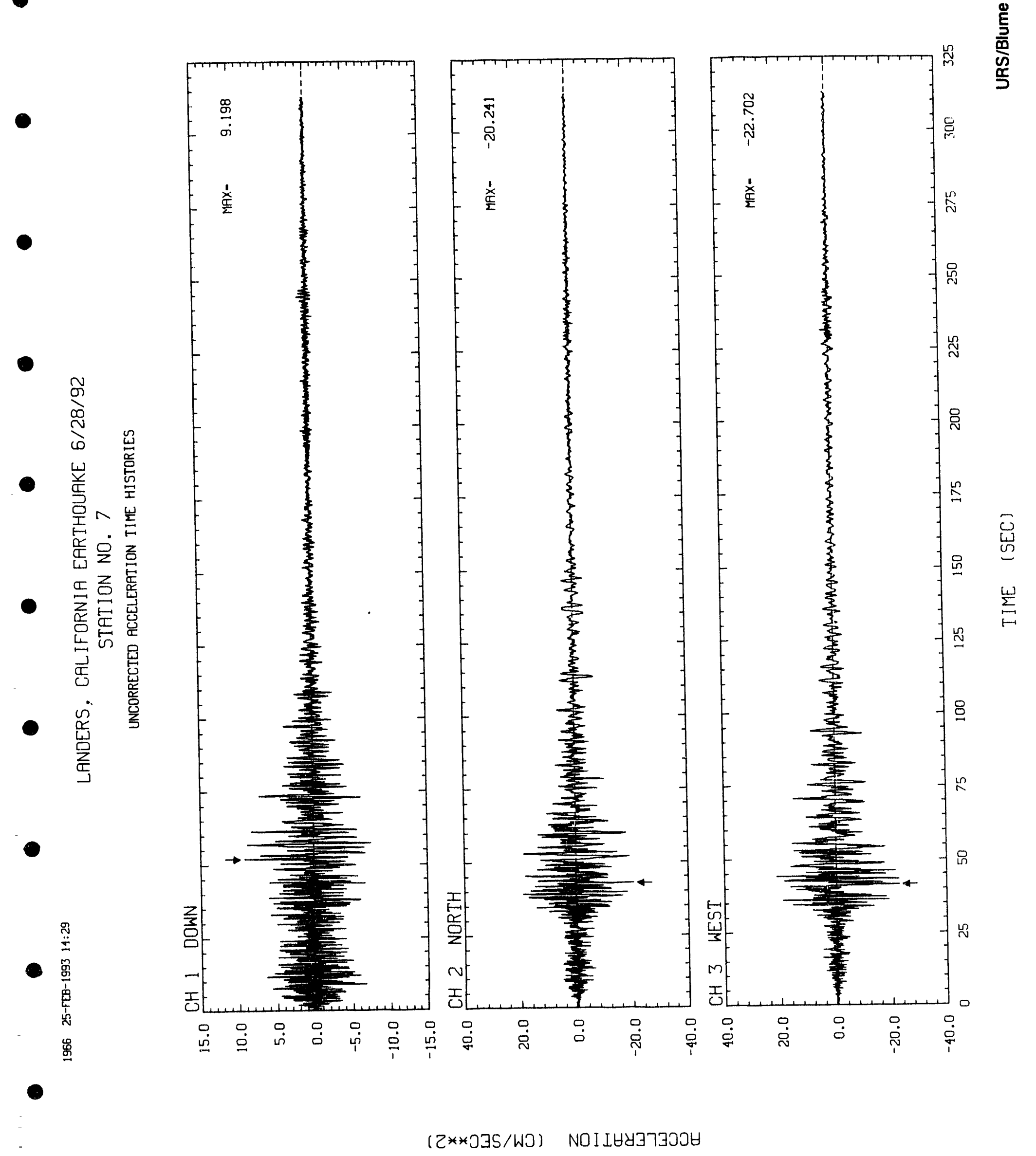




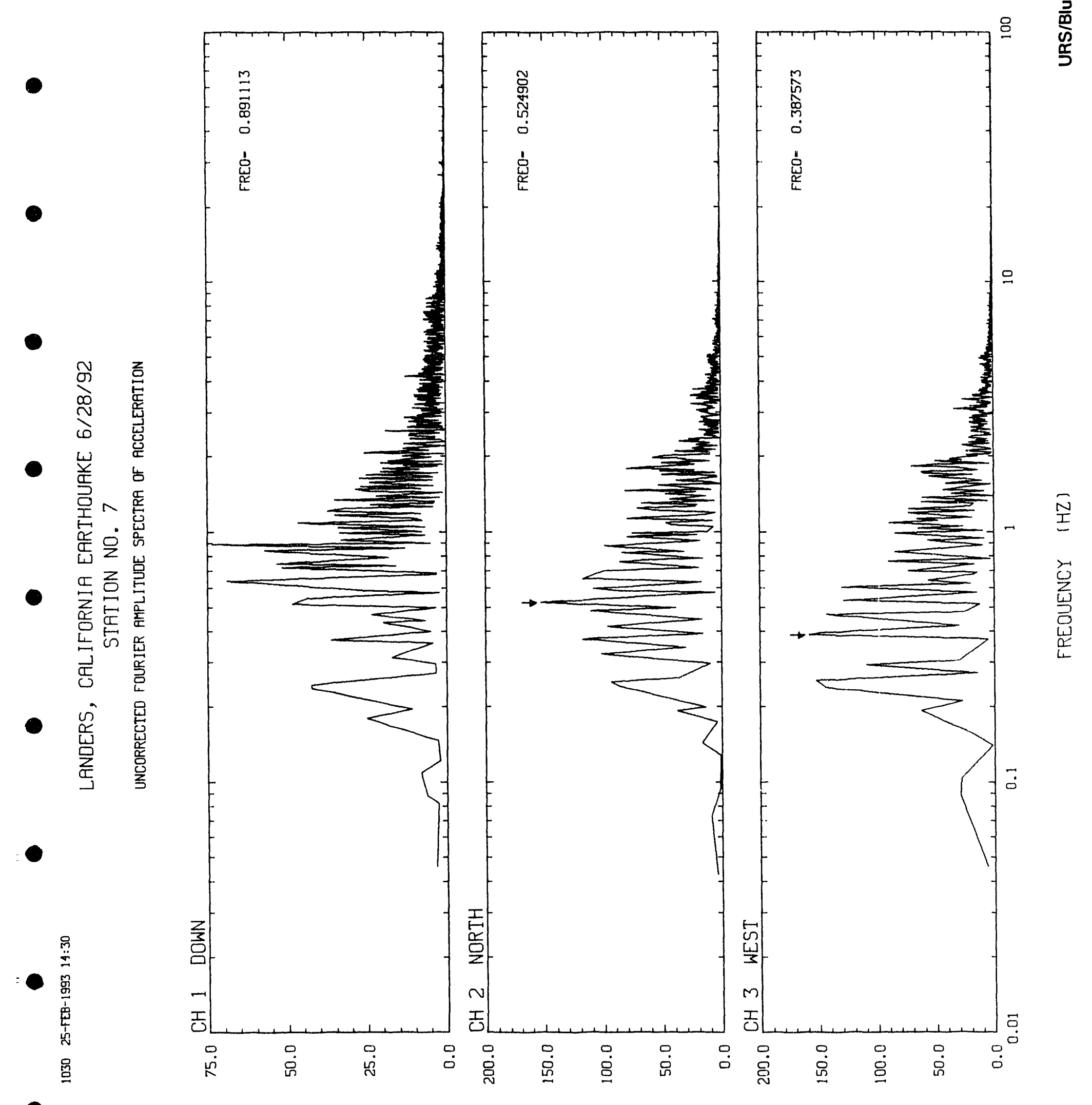

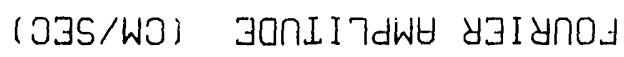




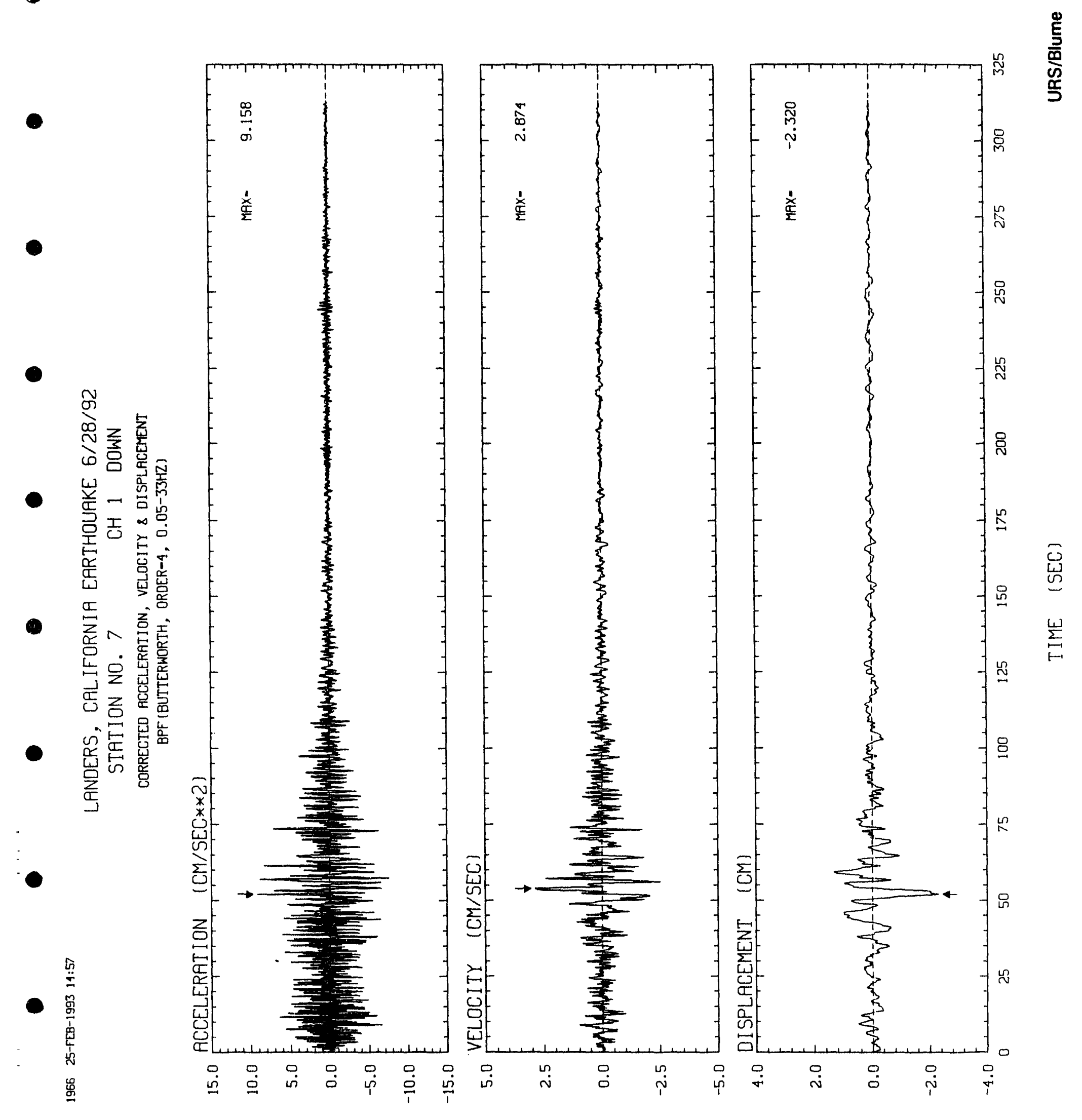

JOחI I 7dWH 


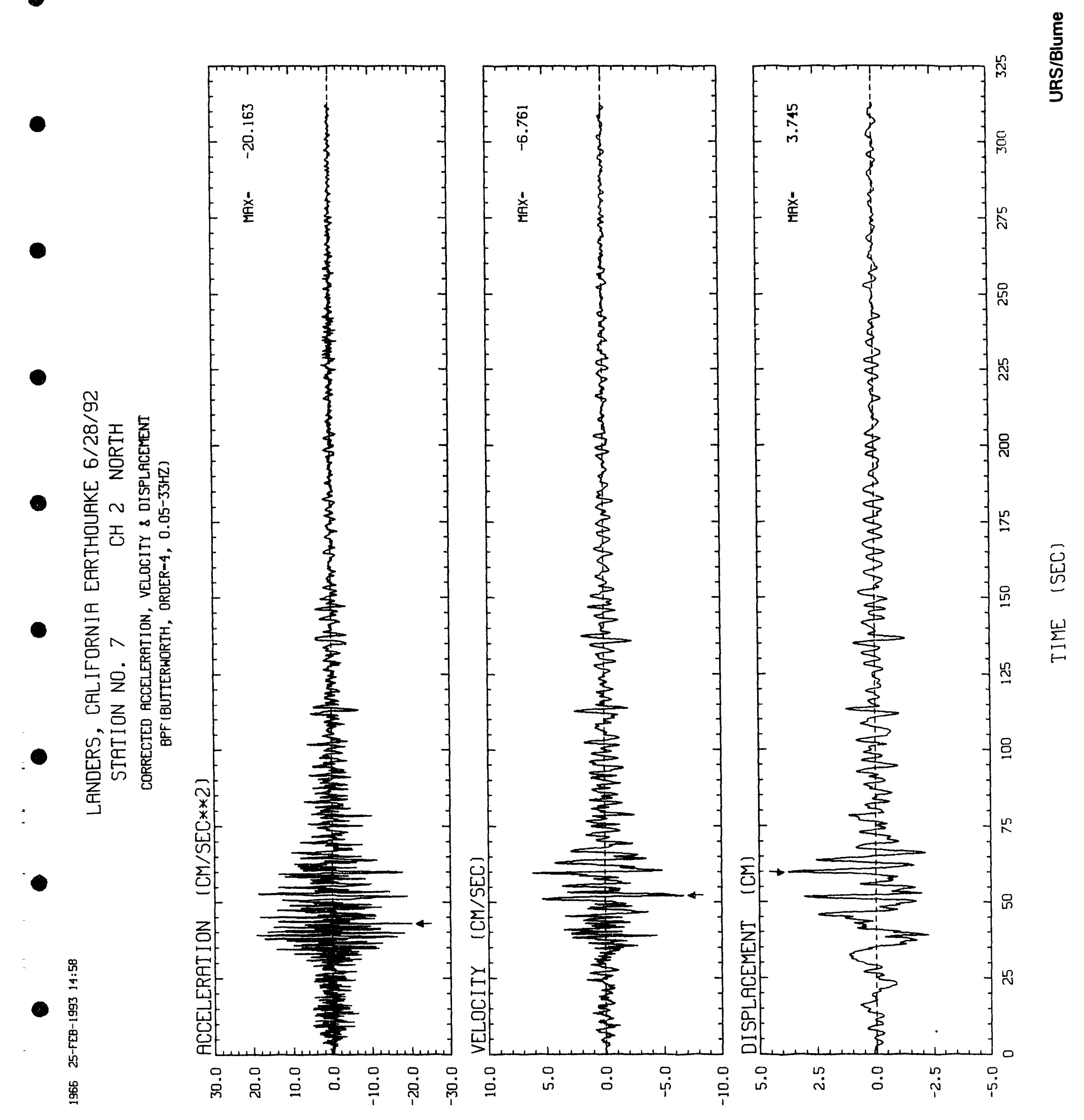



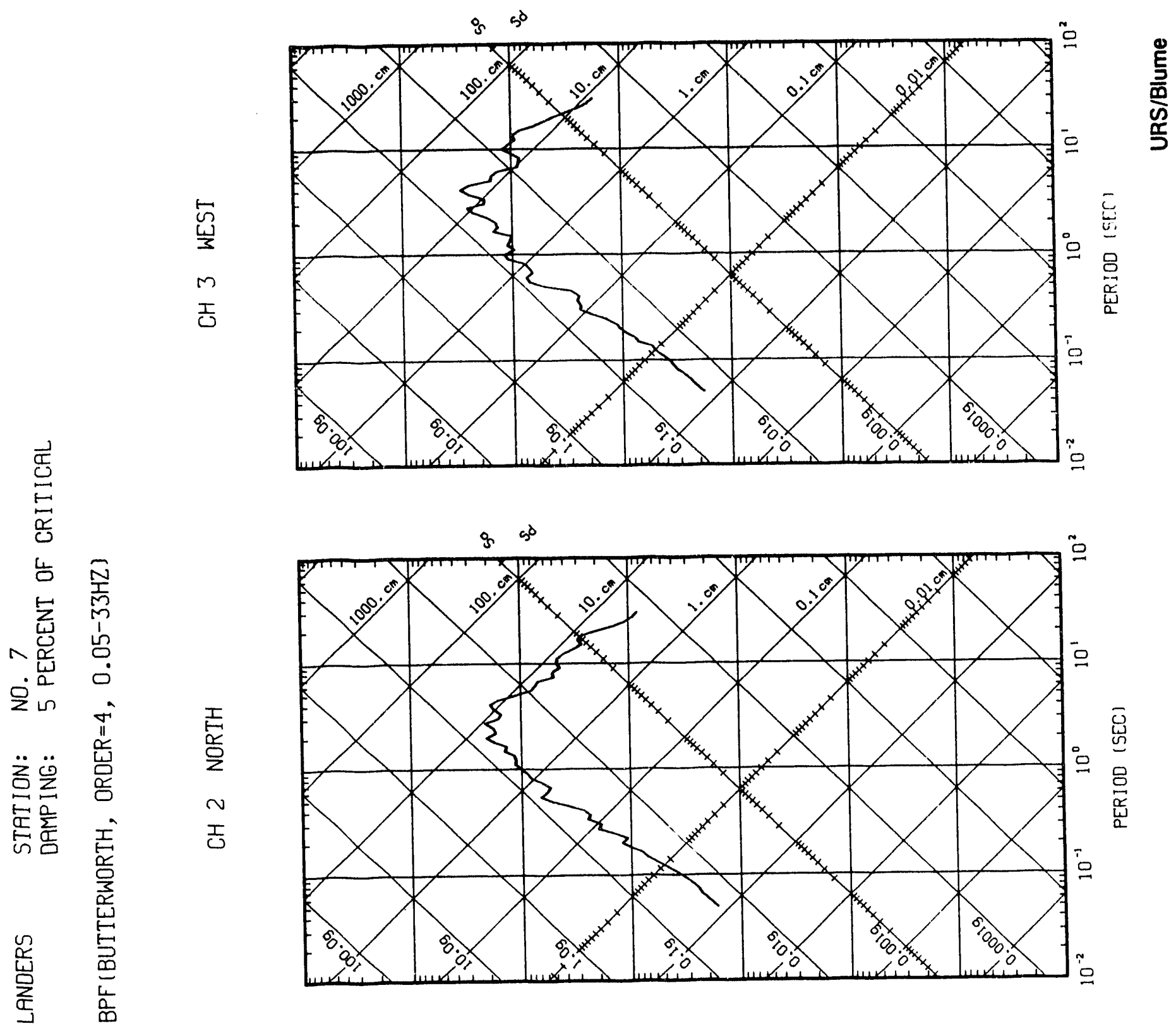

苍空

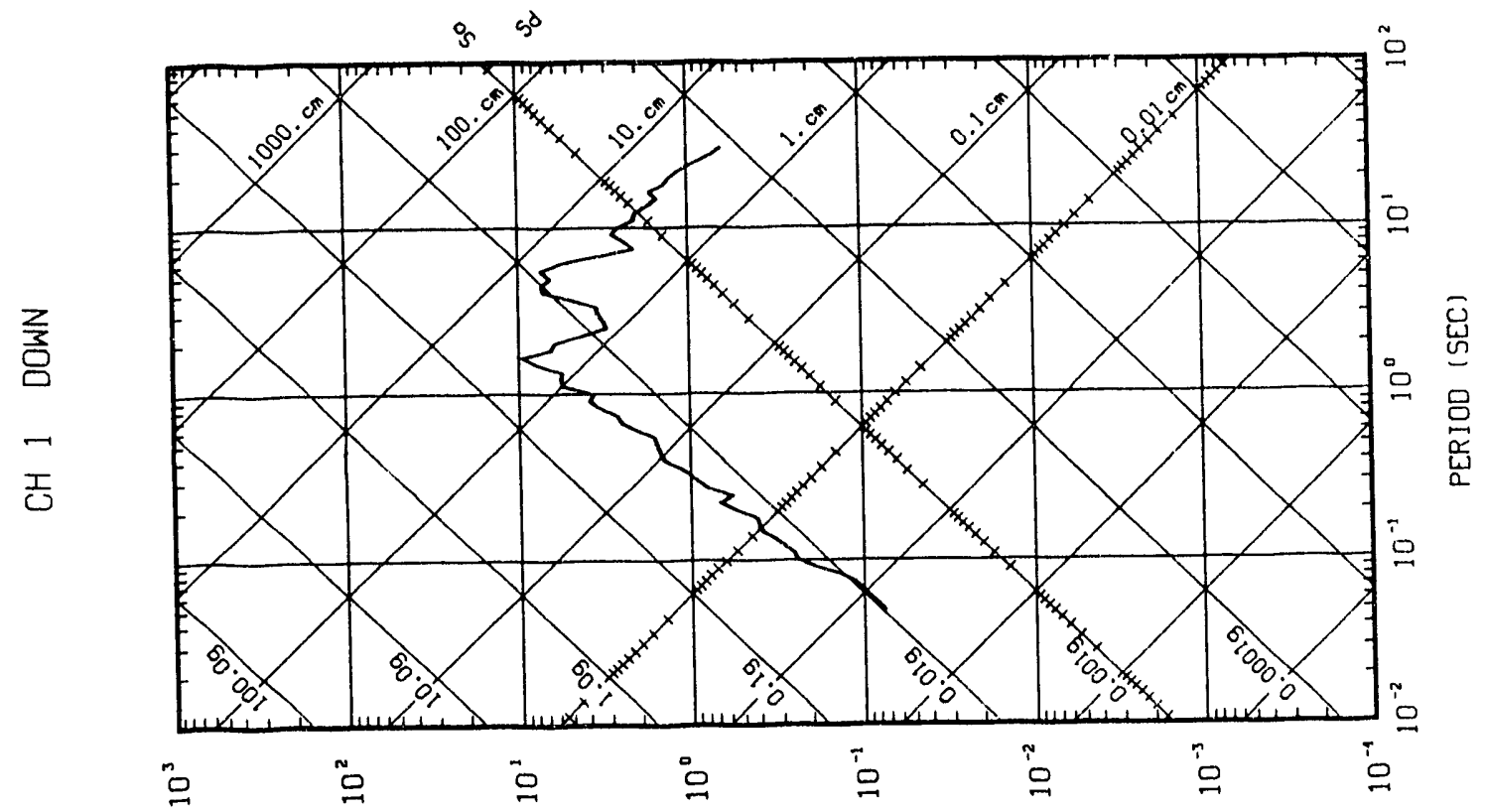

(OJS/WJ) ^S 'XLIJOTIA JSNOdSIY OONJSd 
0

$\infty$

ำ

으 


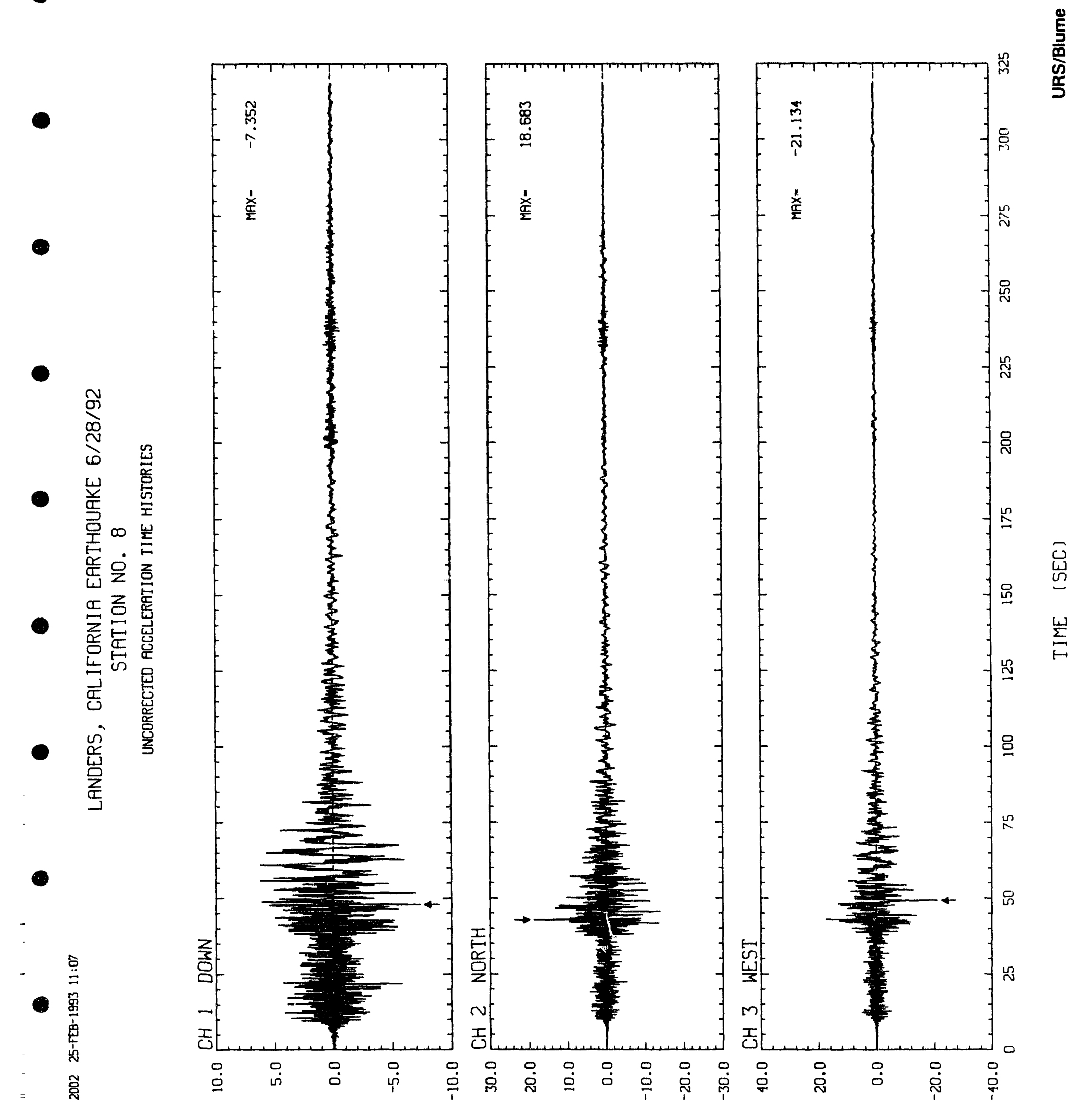

(2**035/WO) NOII I4 $37303 H$ 


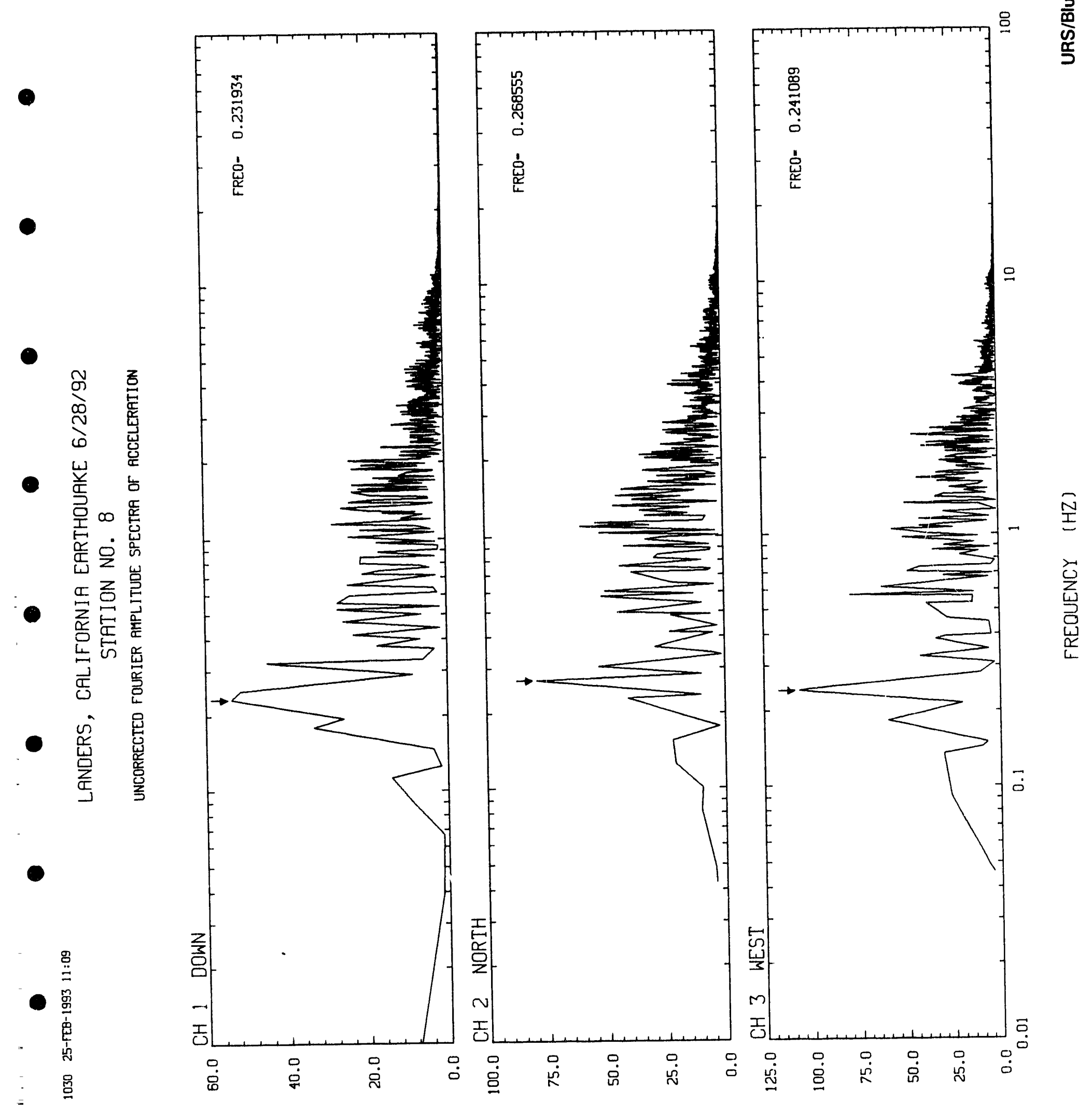

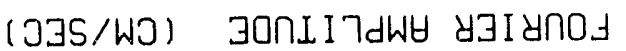




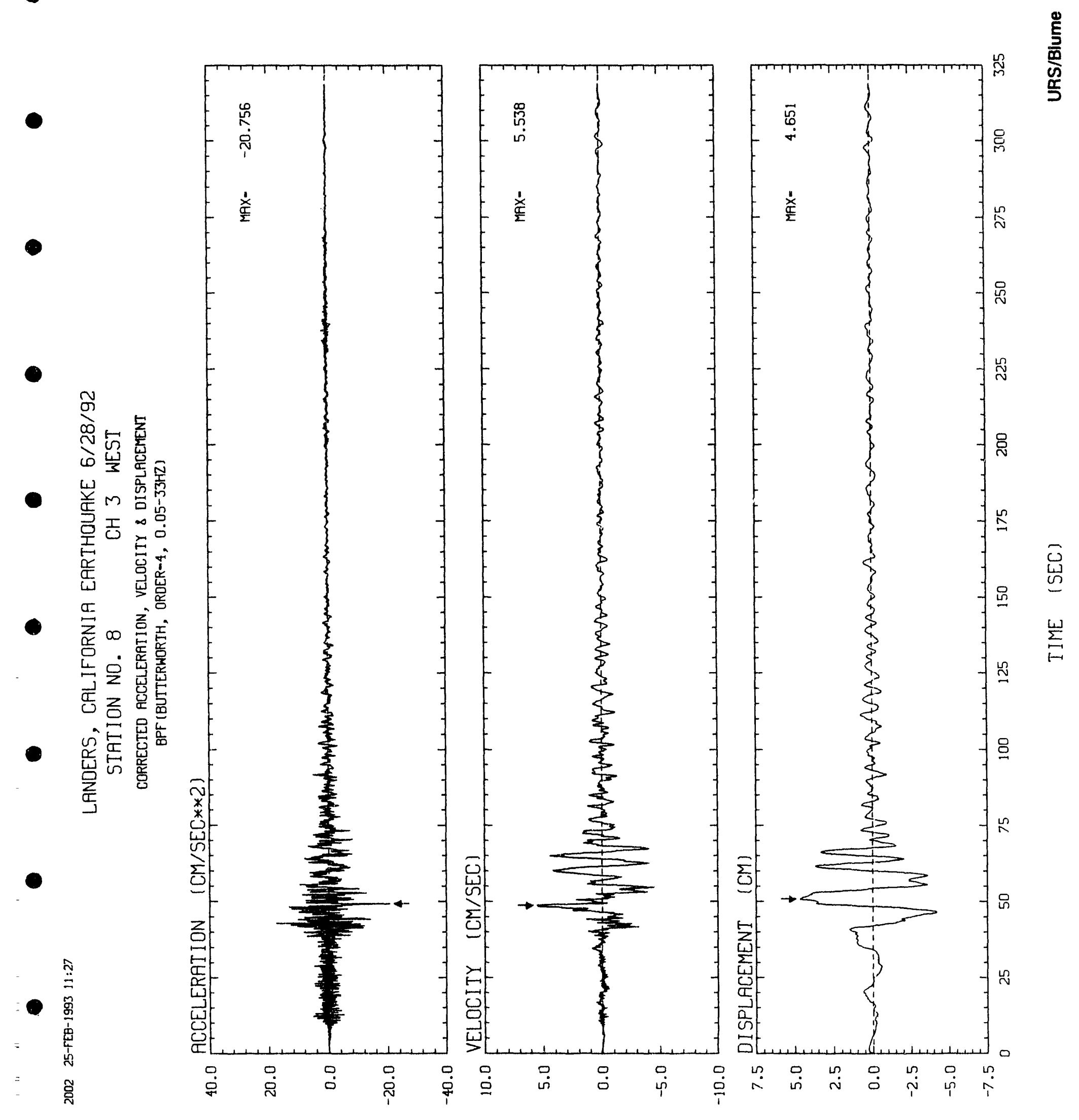



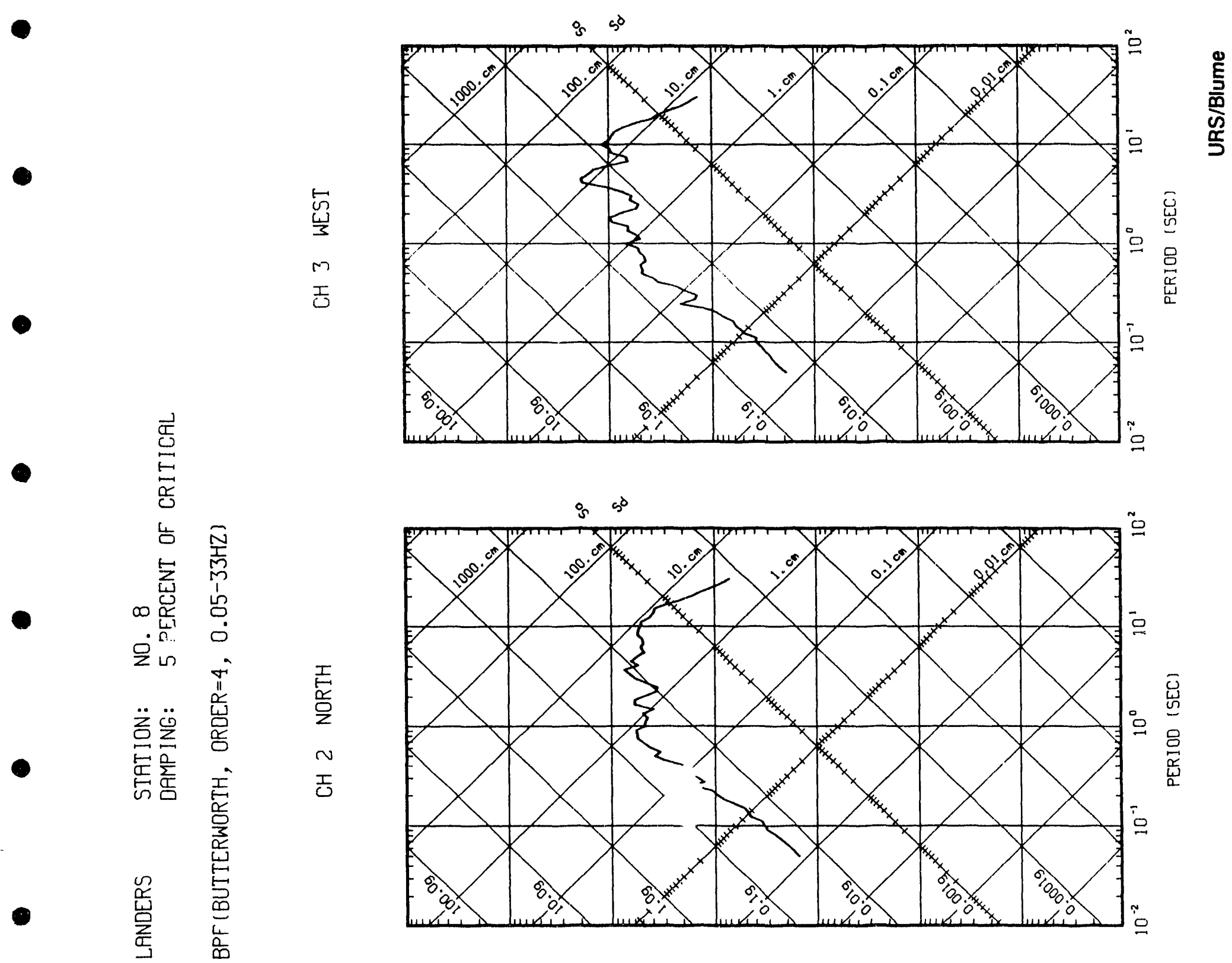

点莣忢

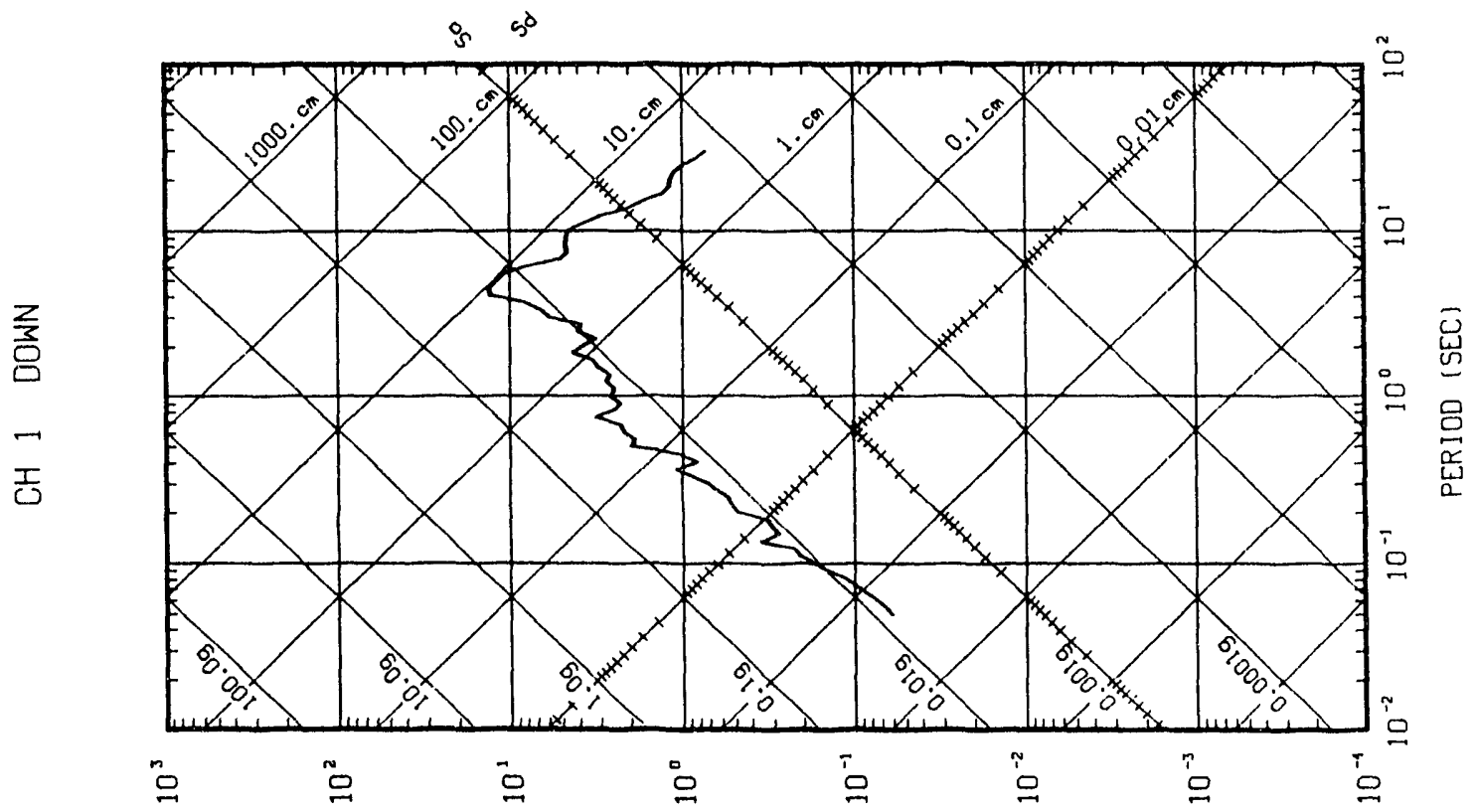

(J3S/WO) ^S 'XIIJOTJA JSNOdSJY OONJSd 


$$
4+1
$$




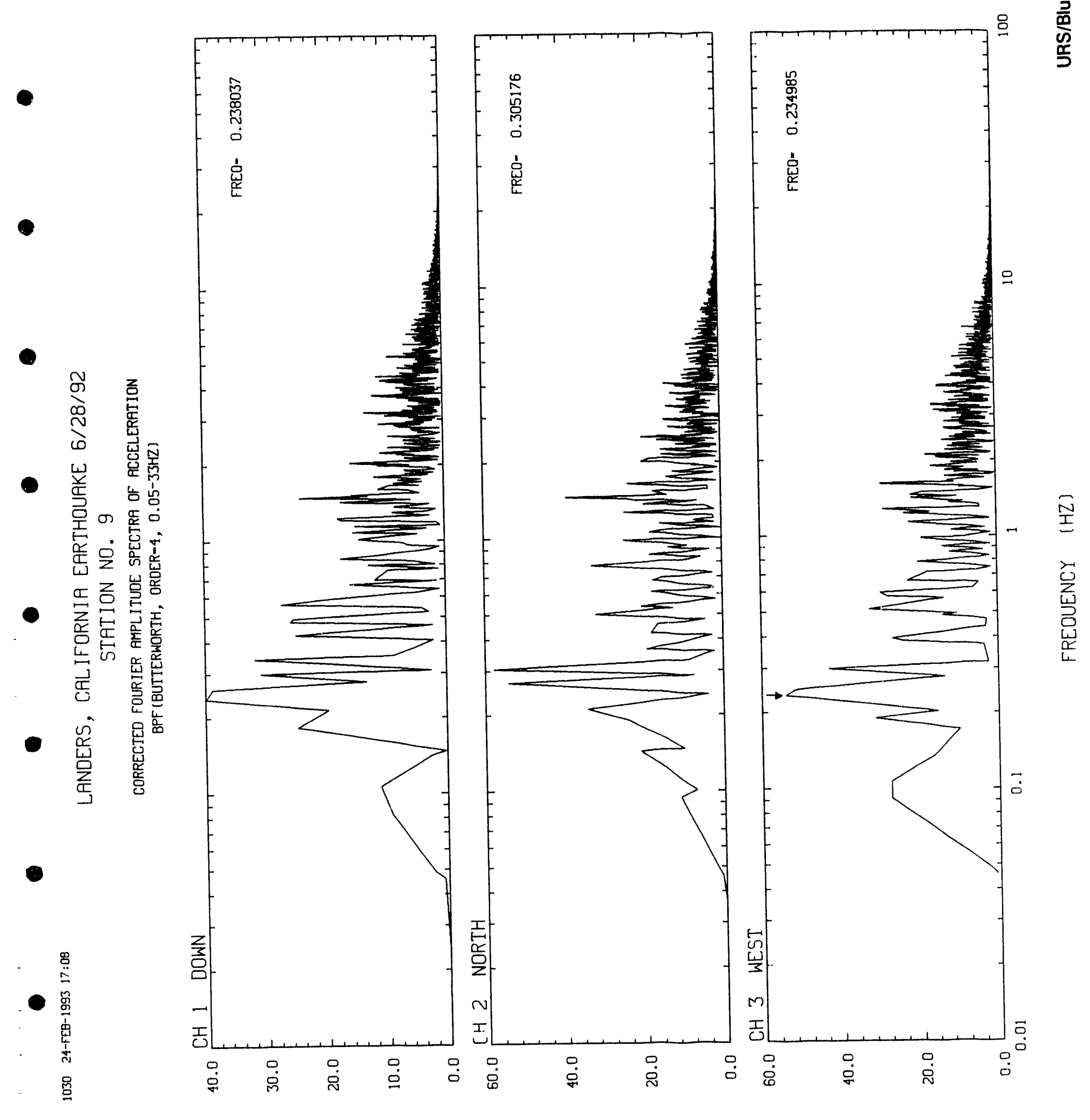

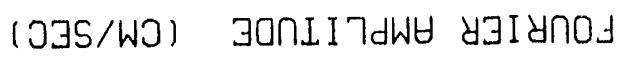




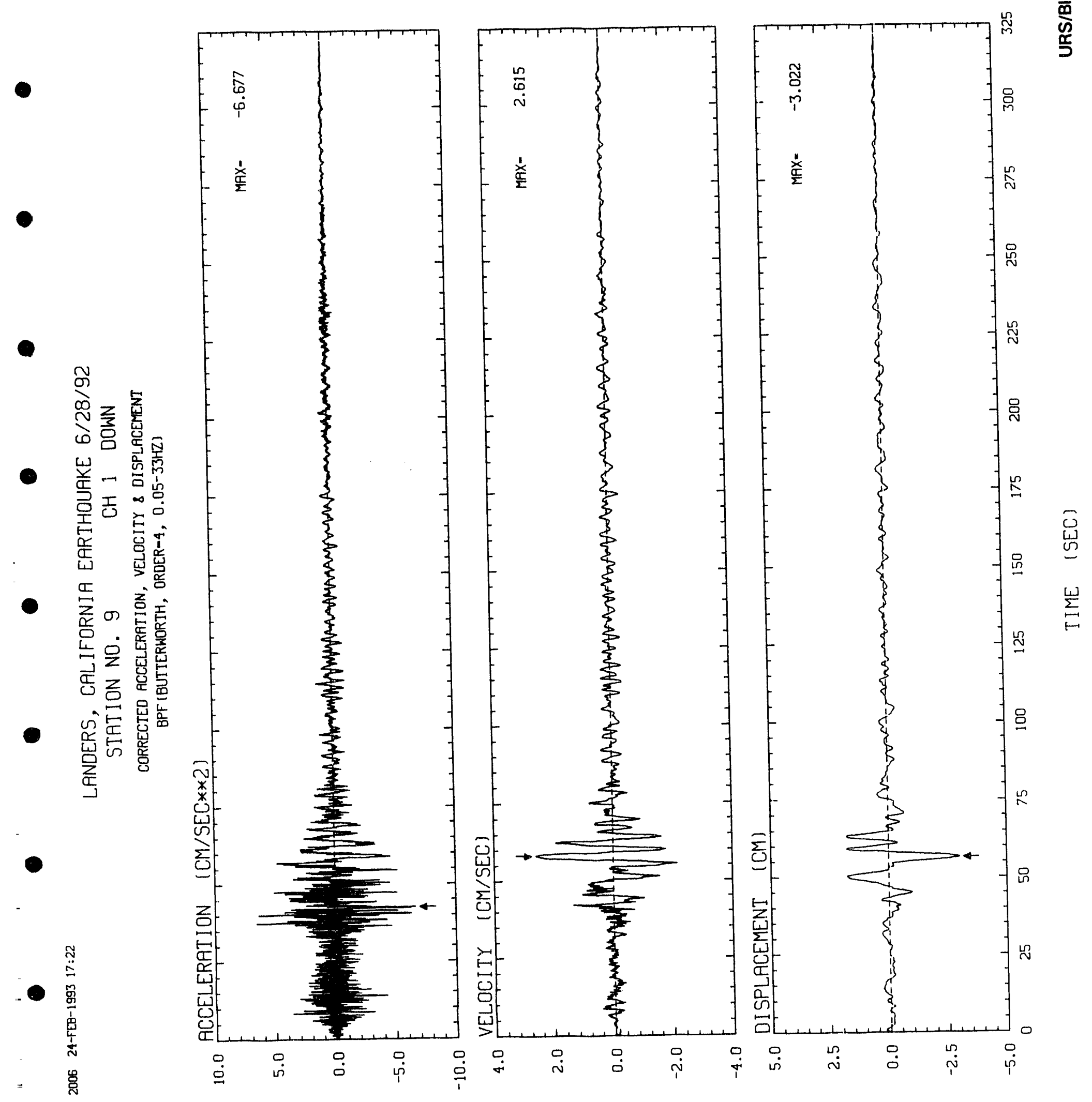

JanI I 7dW 


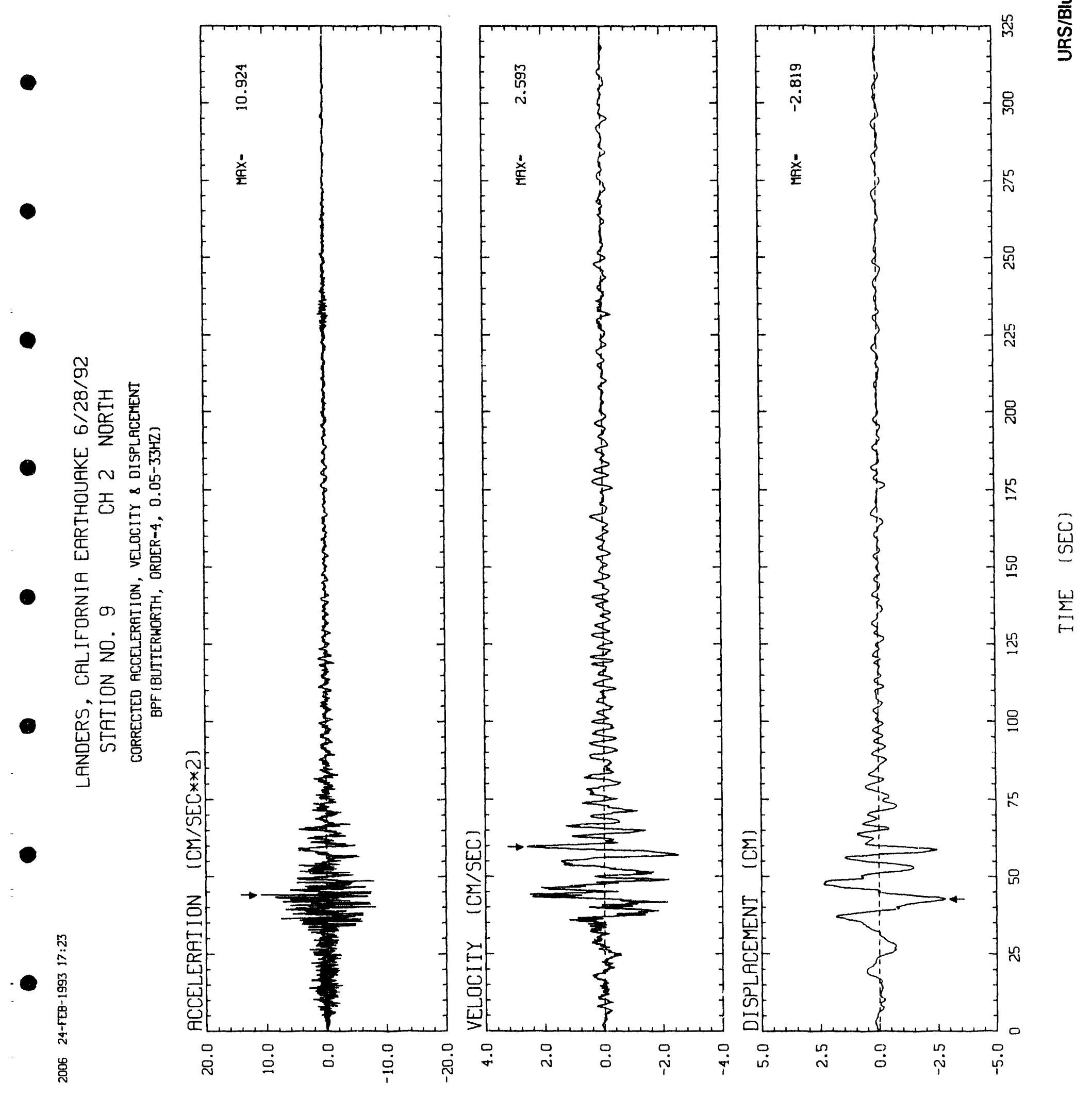




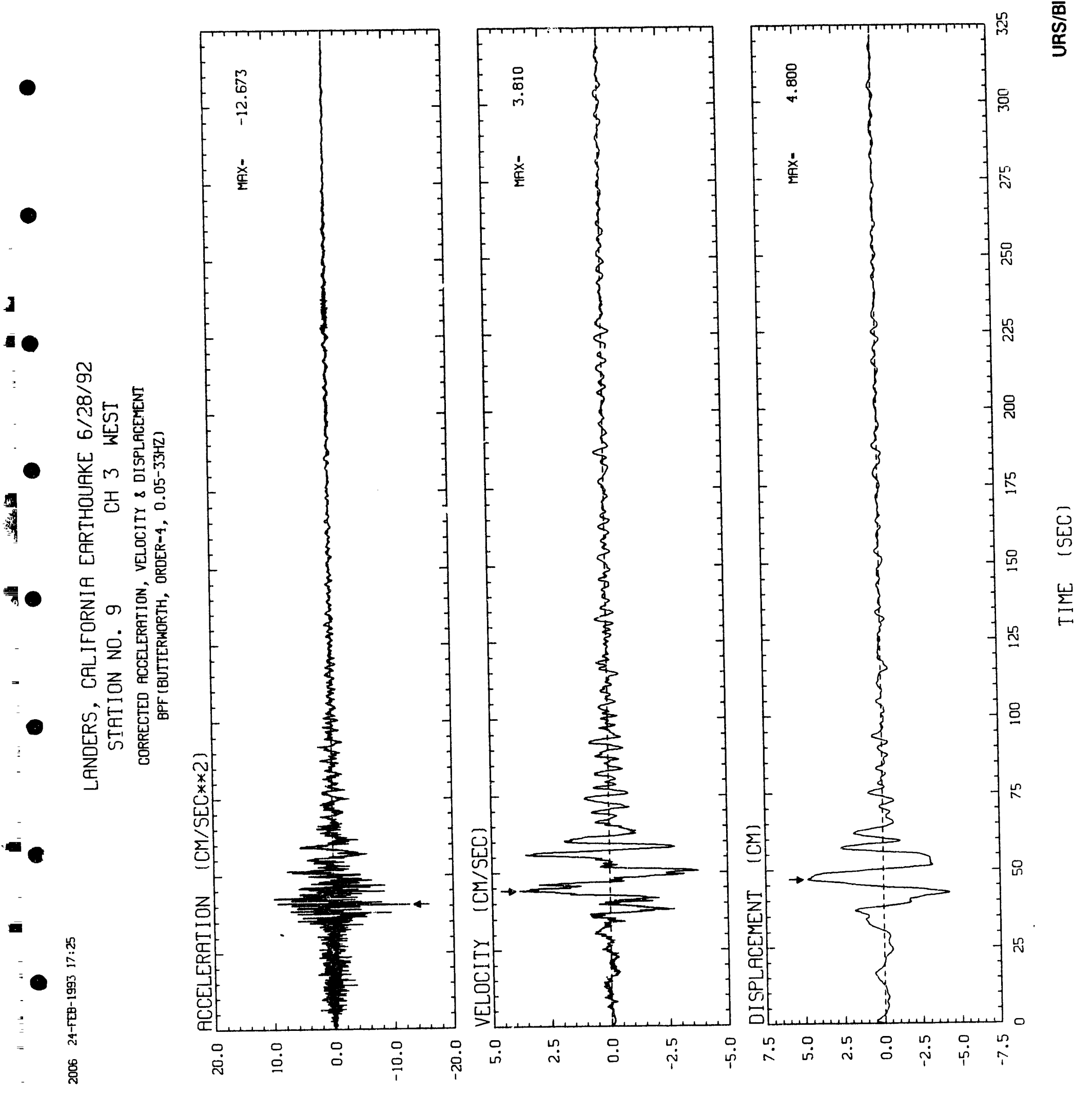

30กI I רdWH 


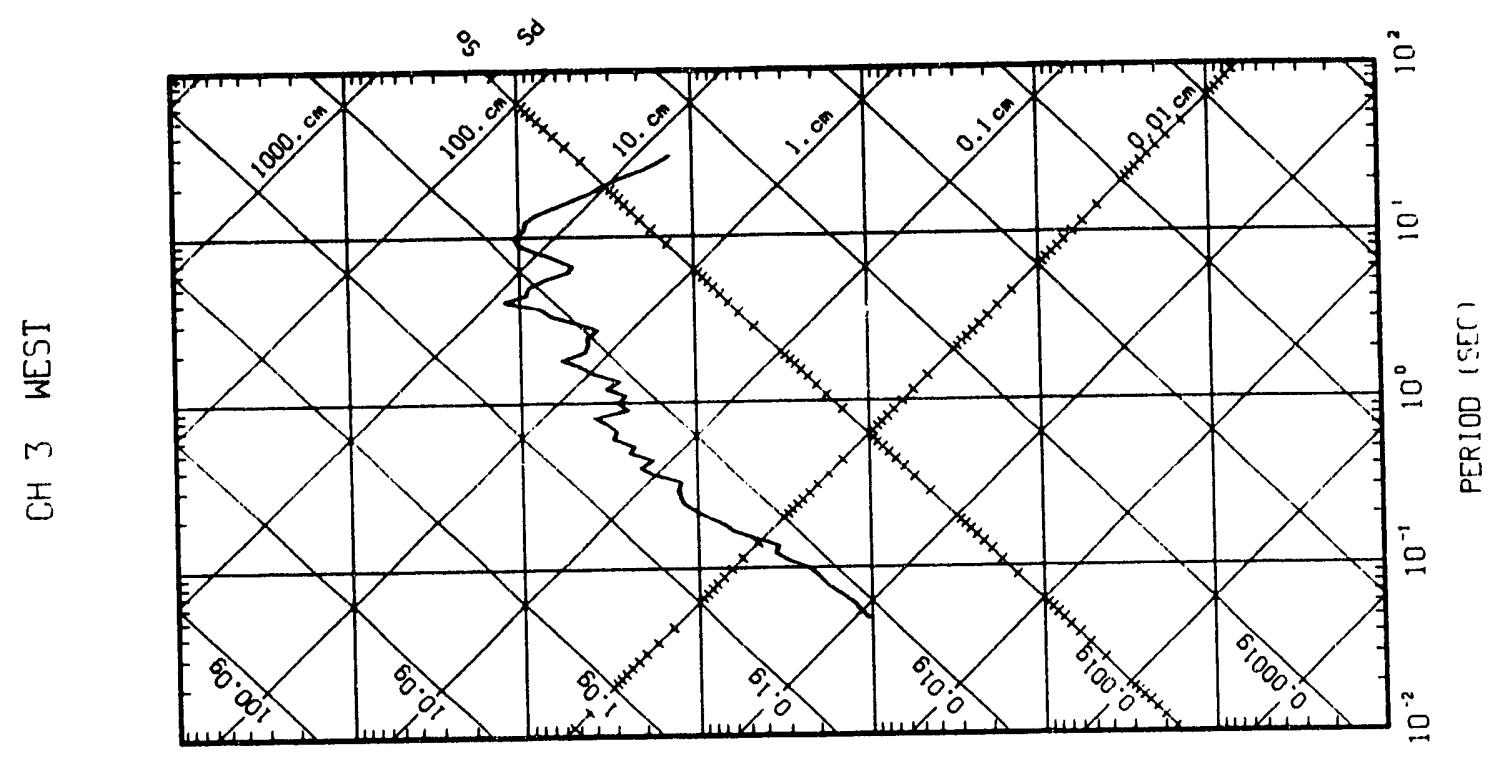

돌

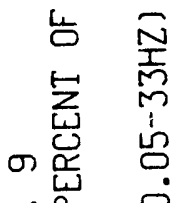

这

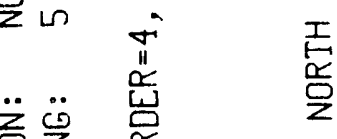

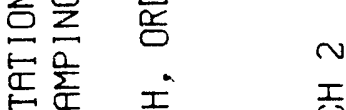

灾吉声吉

号

$\sum_{\omega} \underset{\omega}{\stackrel{i}{\omega}}$
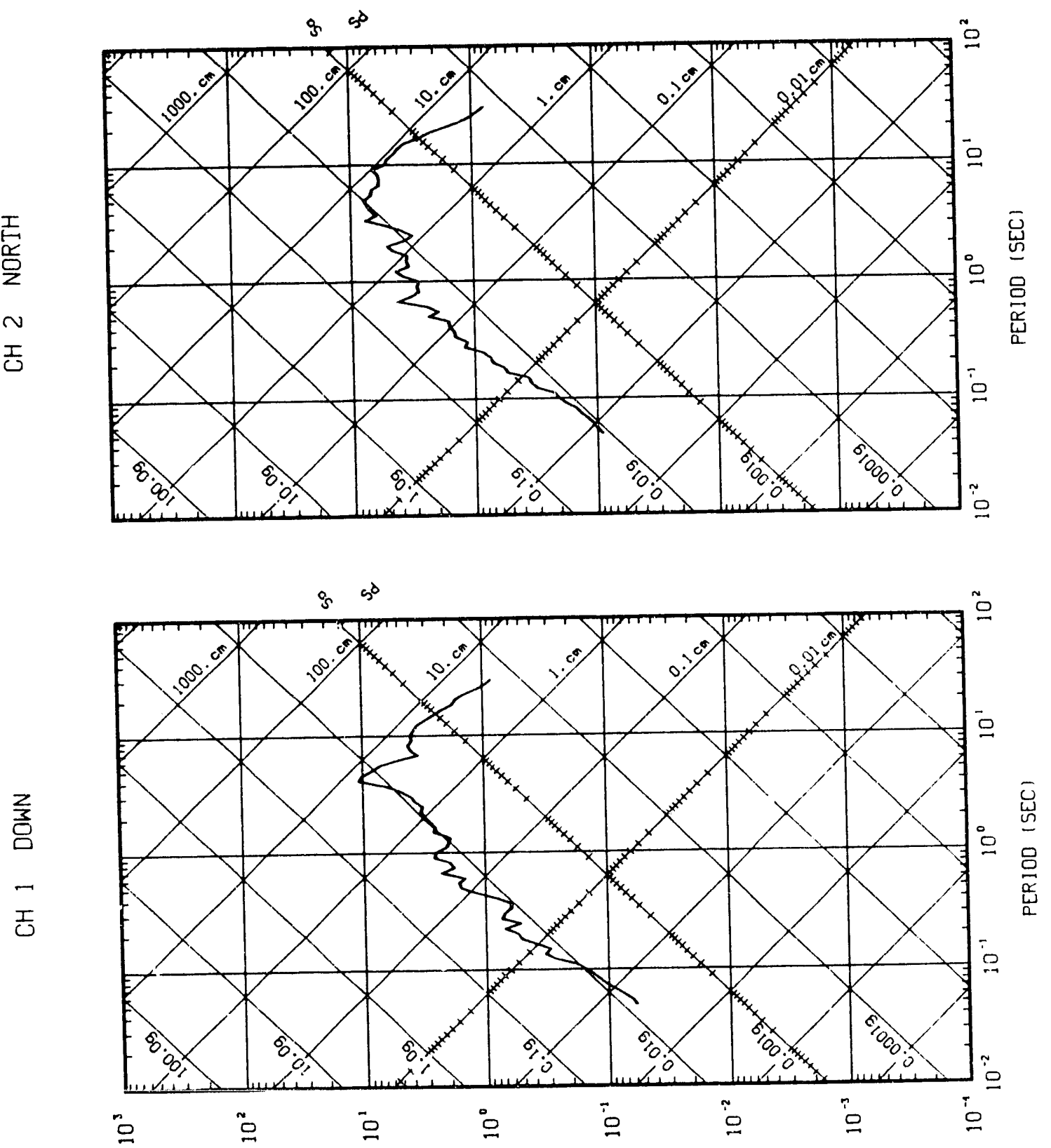

(33S/WJ) ^S ‘ XI10073^ 3SNOdS3y O0n35d 
ㅇ

0

○ 


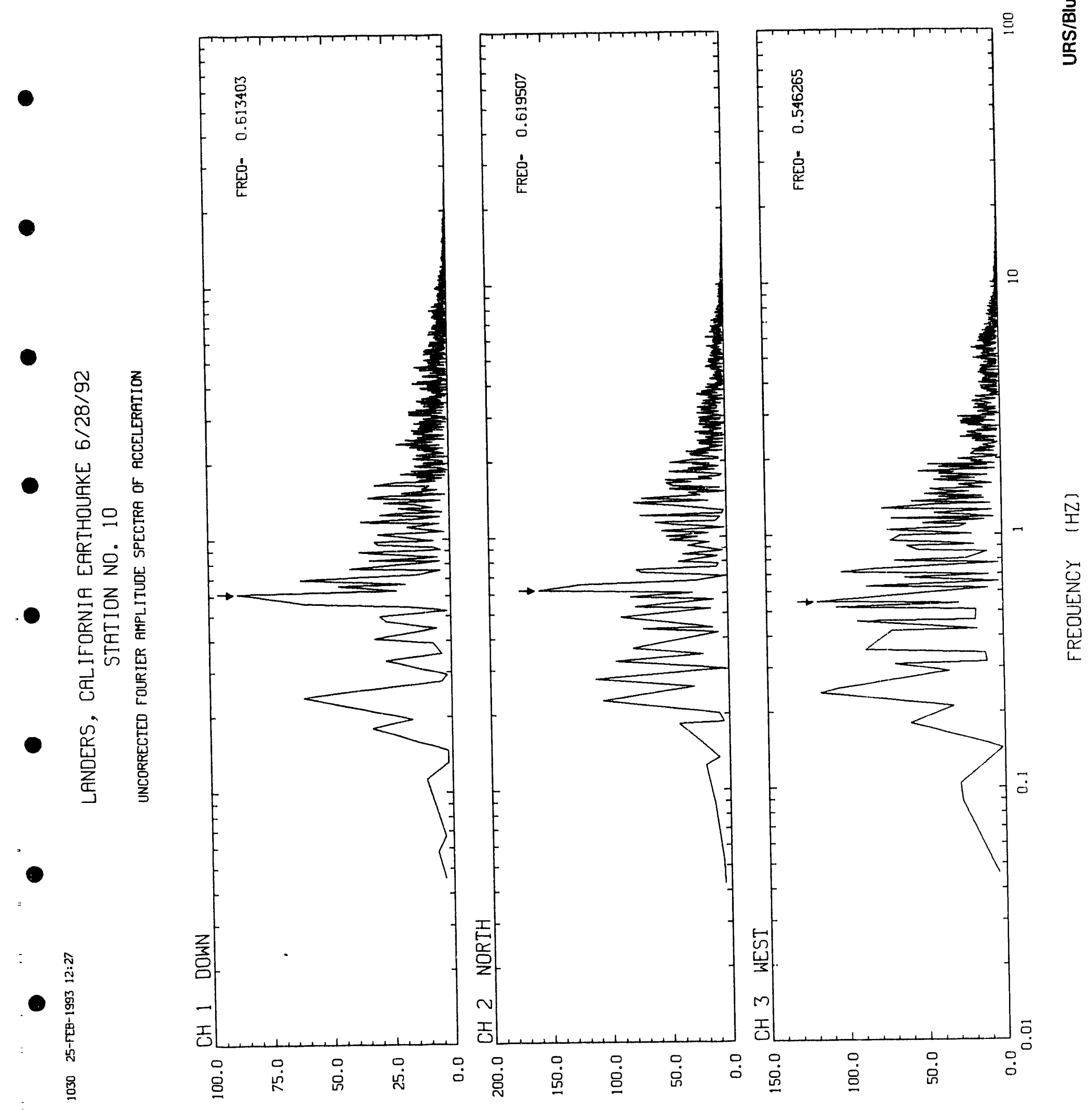

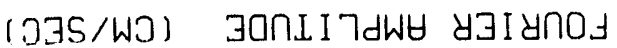




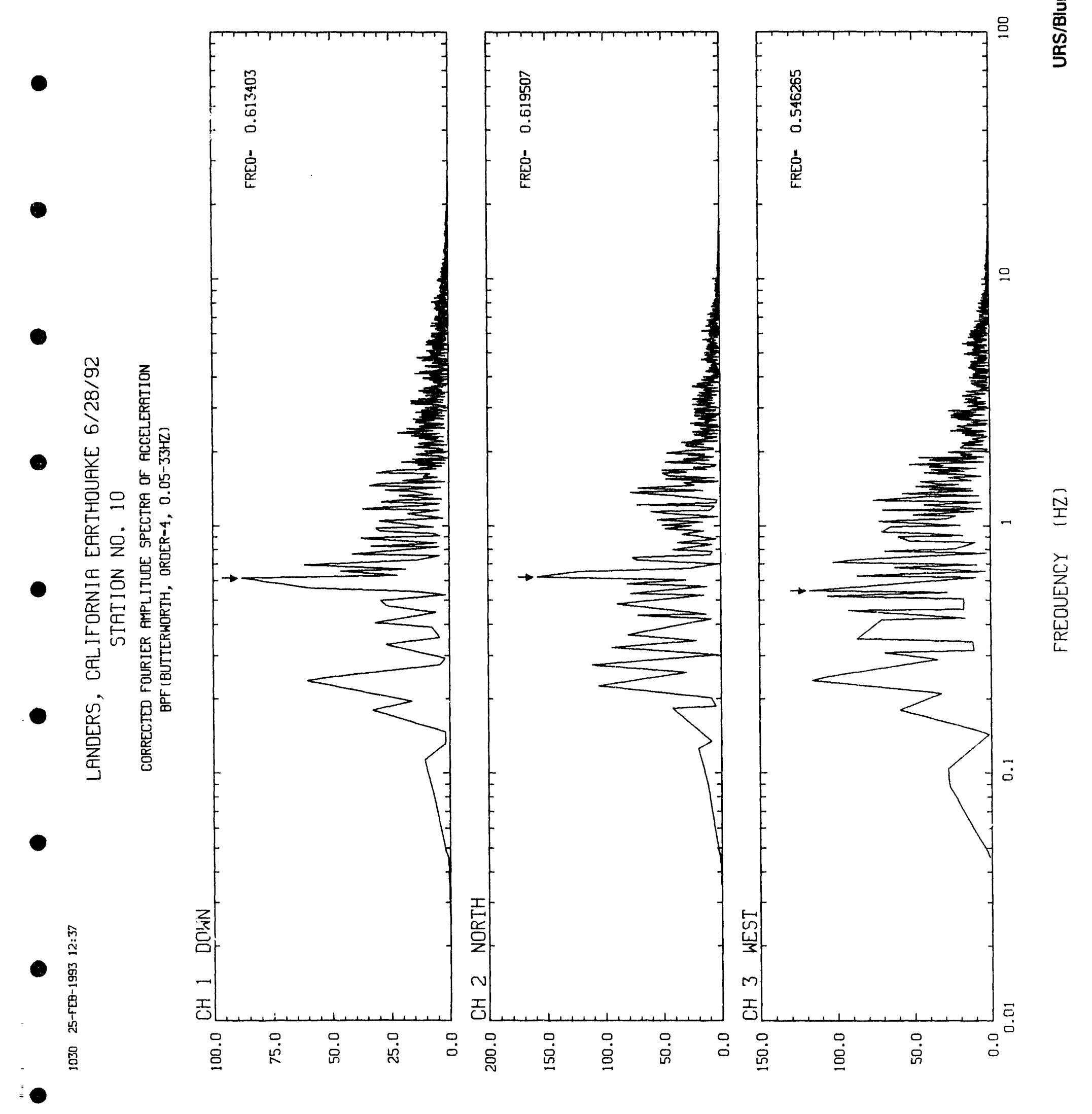

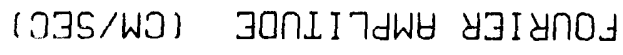




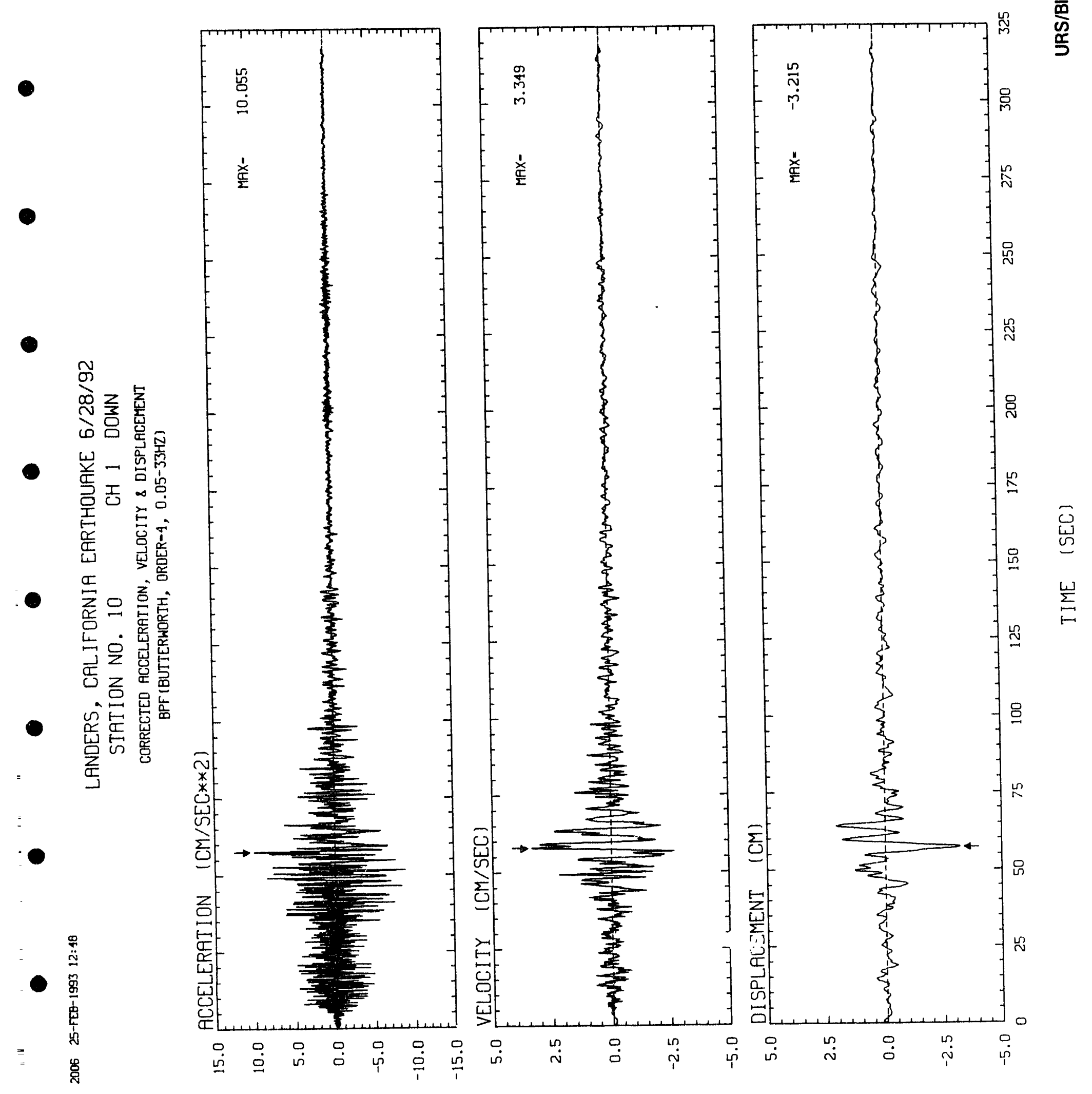



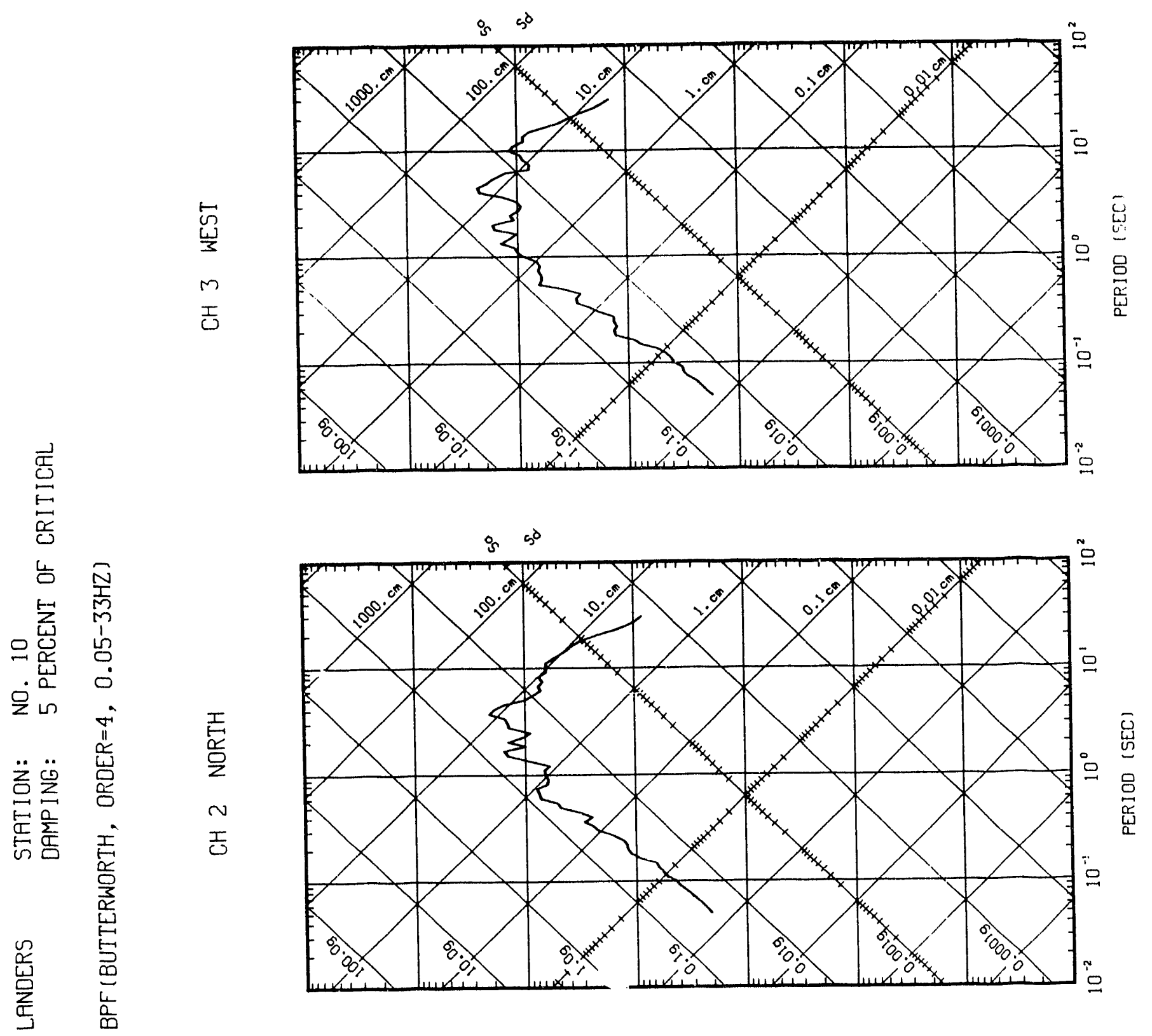

芯点

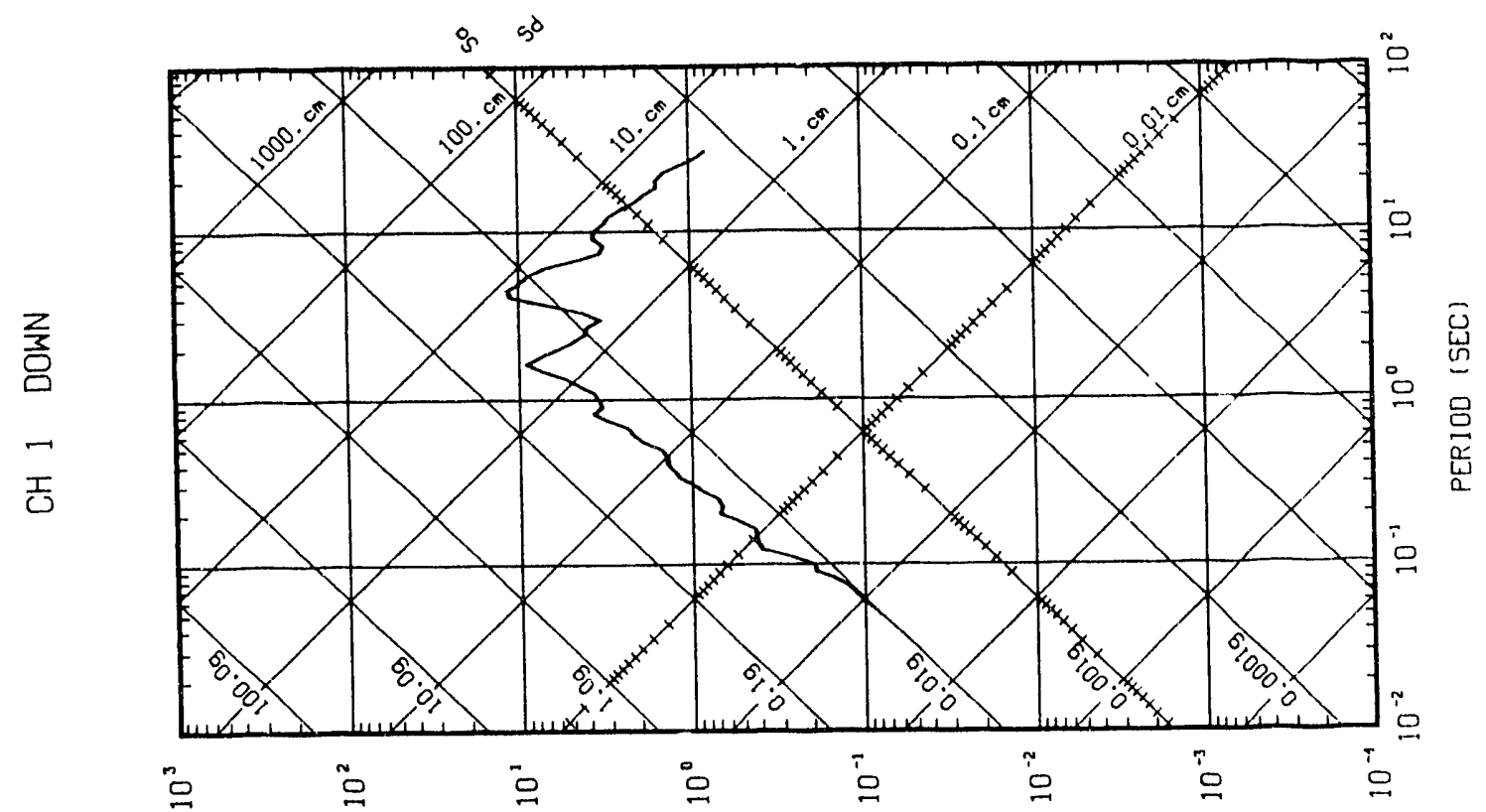

(J3S/WO) ^S “XLIOOTIA ISNOdS3Y OONJSd 


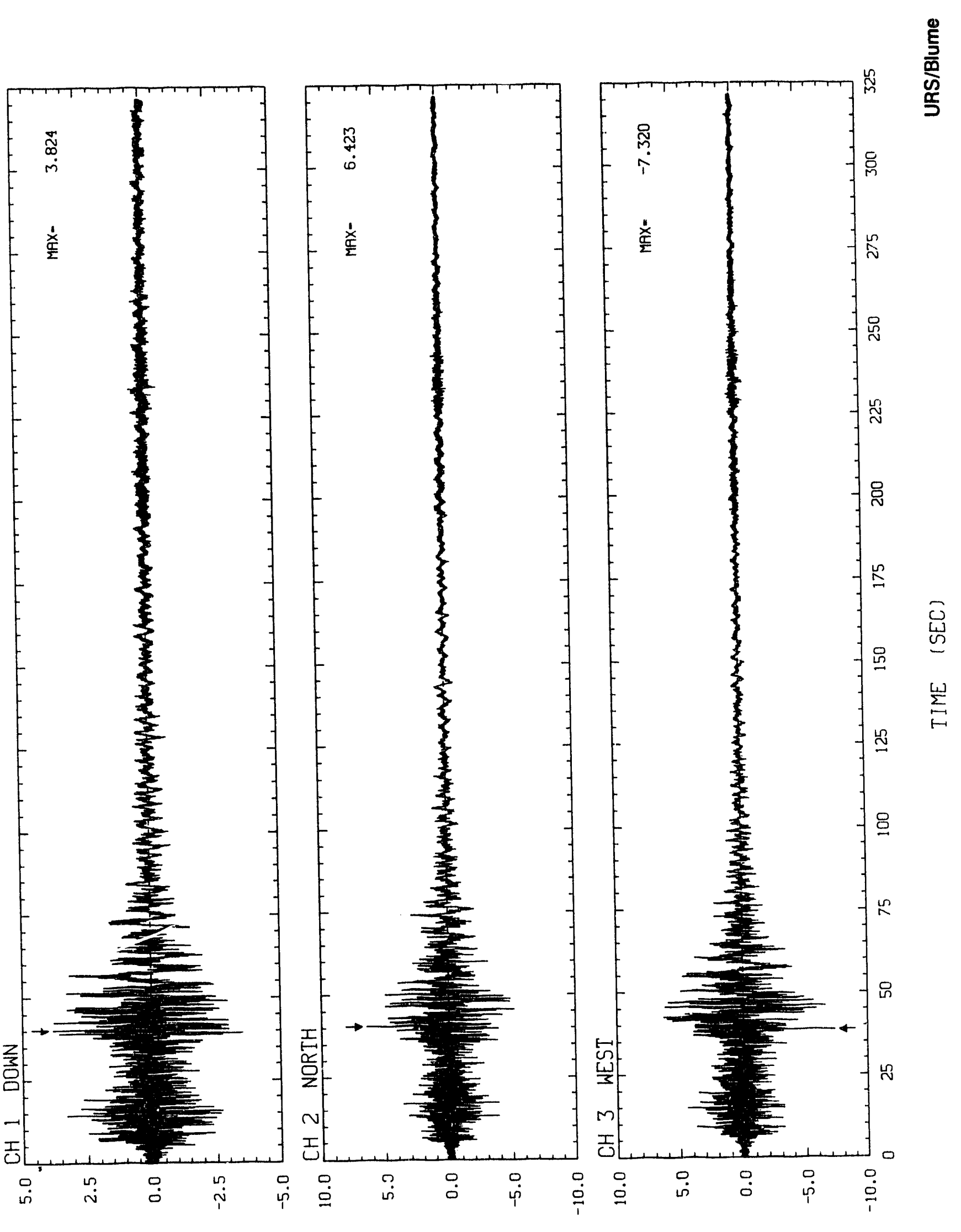

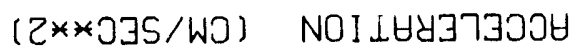




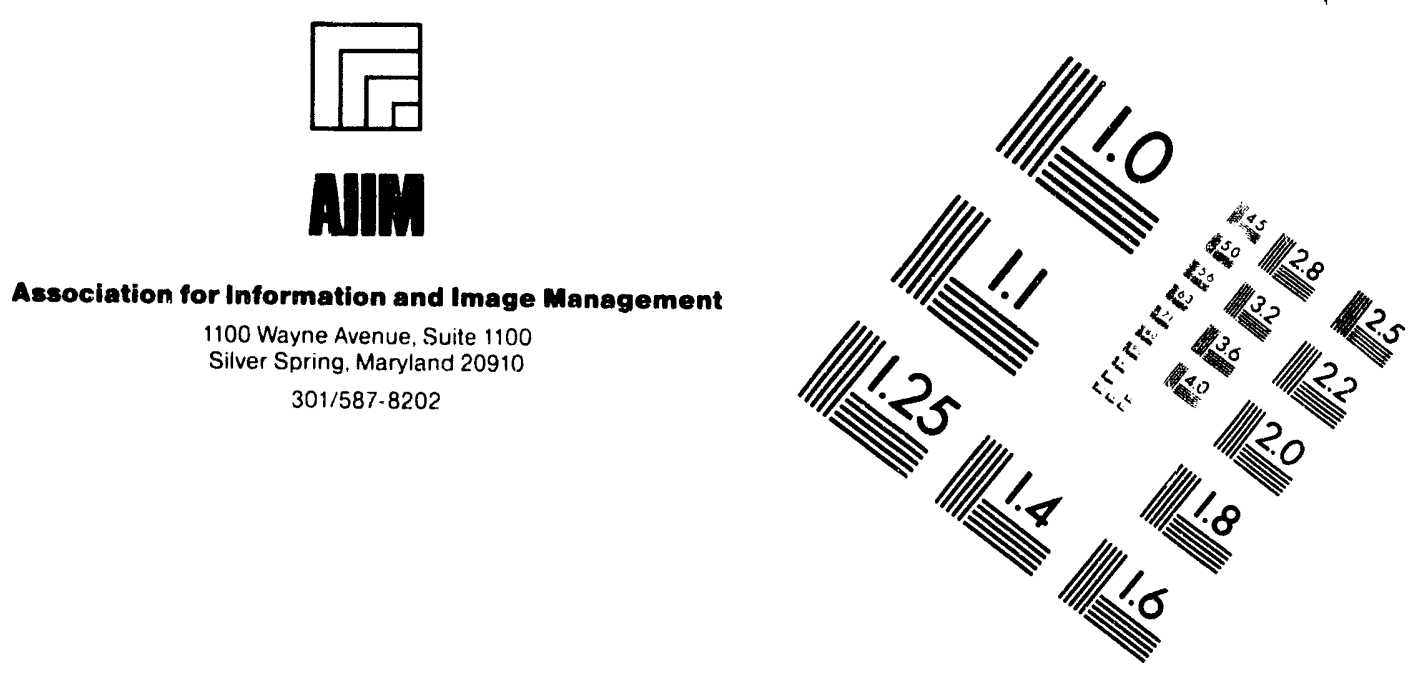

\section{Centimeter}

1m

Inches
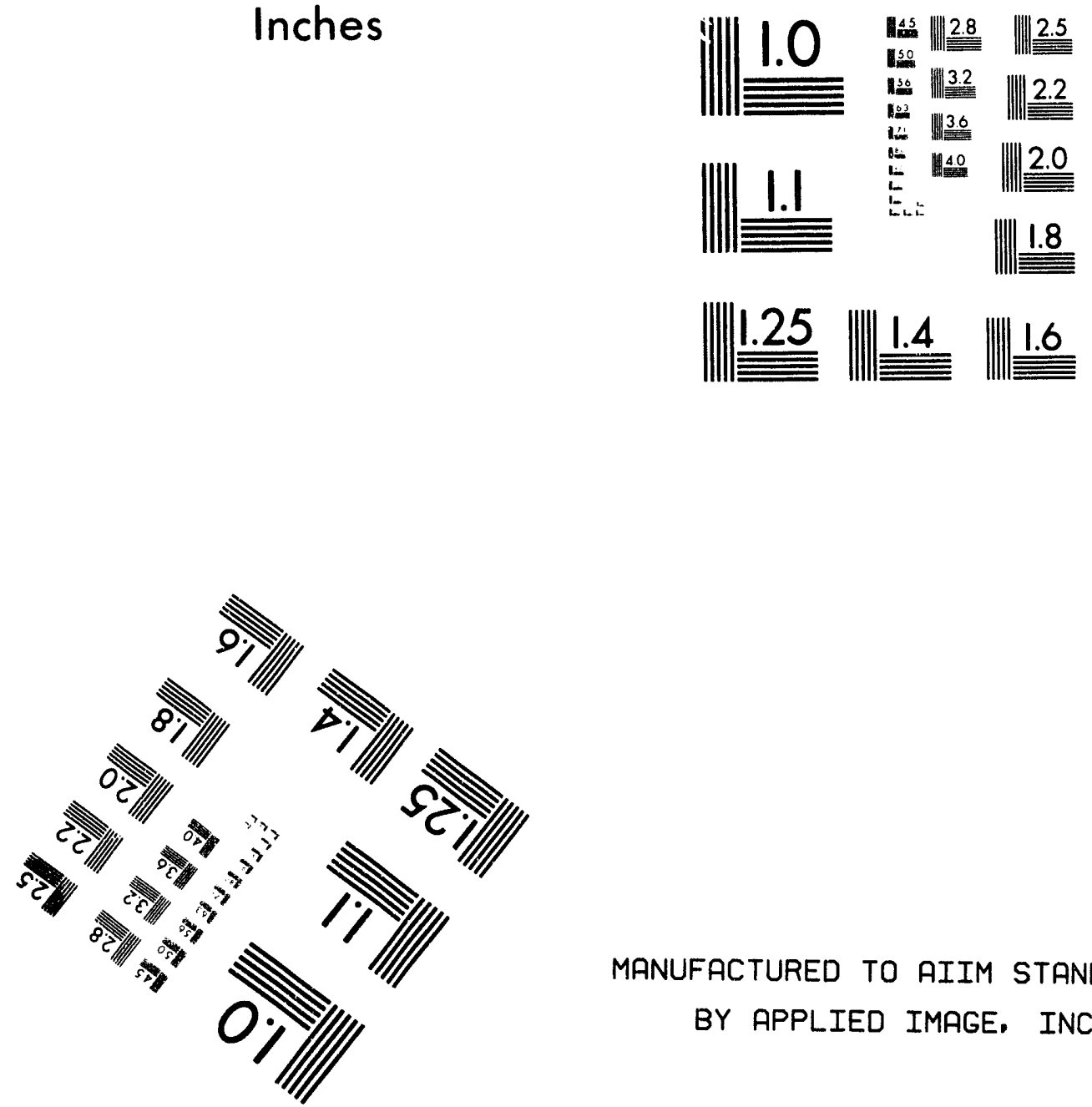

MANUFACTURED TO AIIM STANDARDS

BY APPLIED IMAGE, INC.

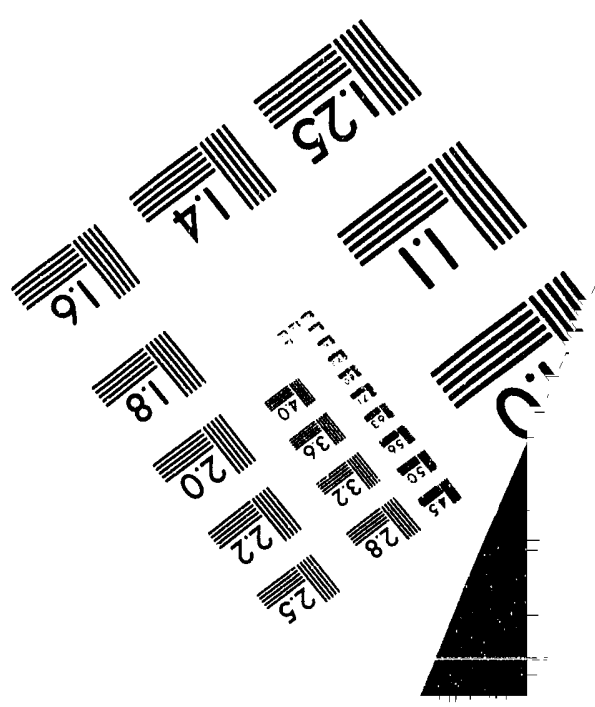



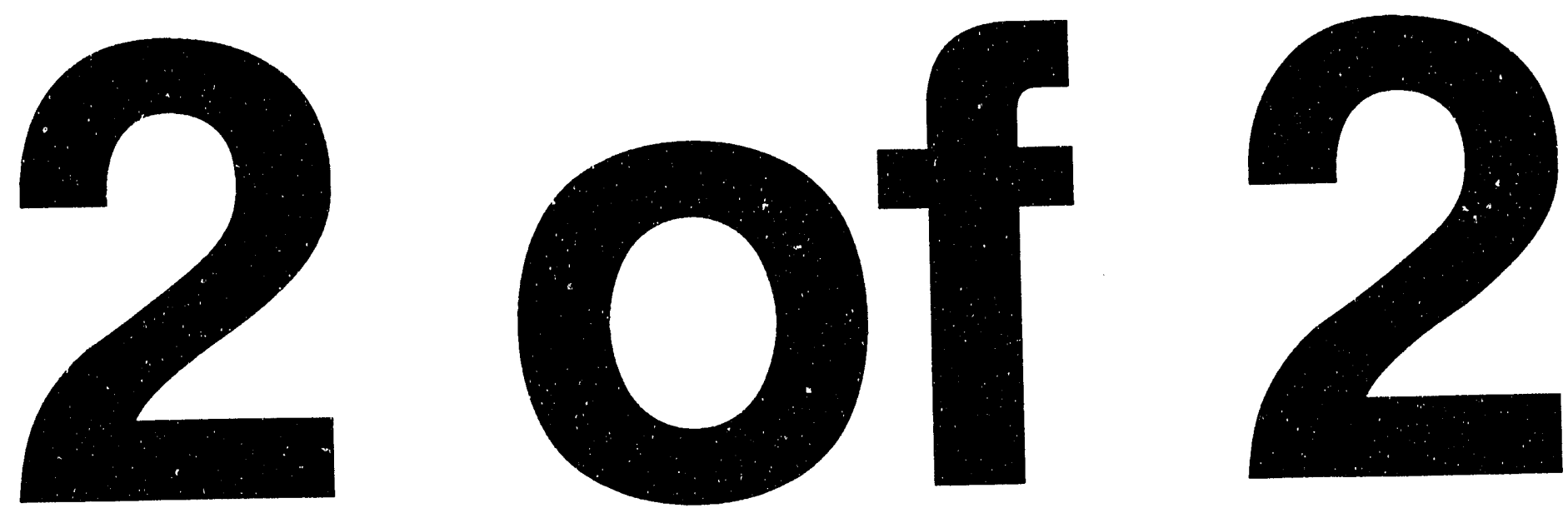



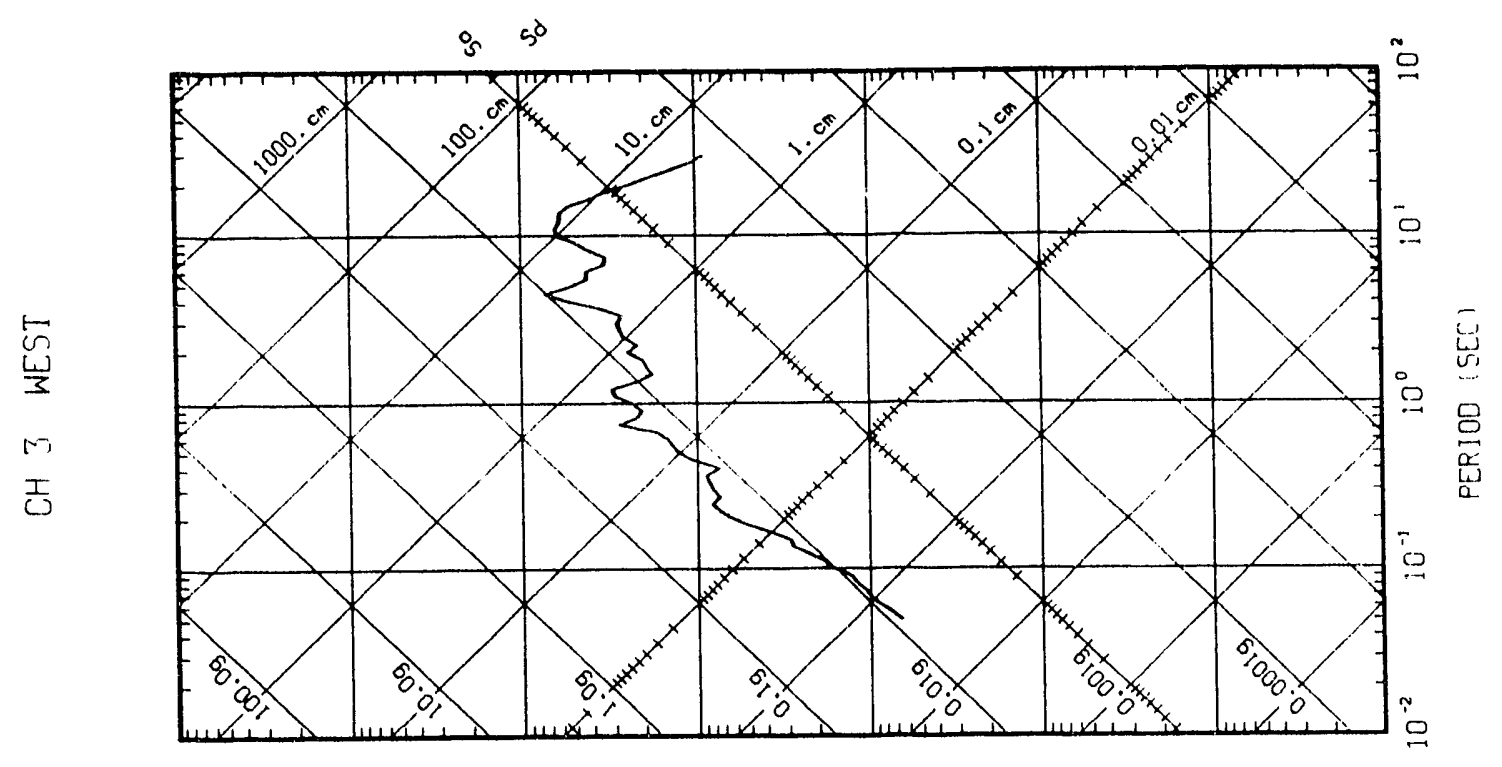

$\frac{\stackrel{0}{5}}{\frac{0}{0}}$
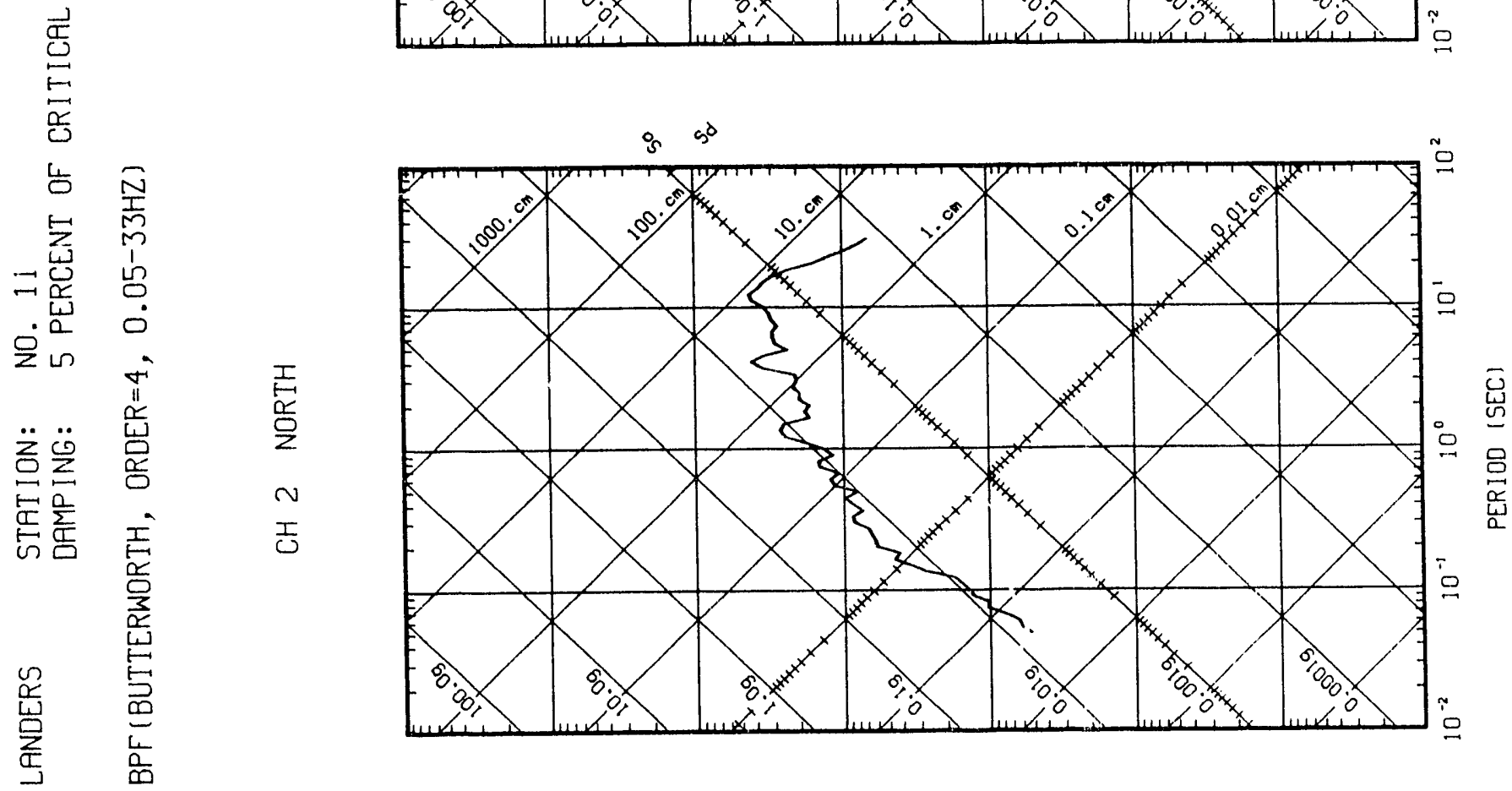

空悹
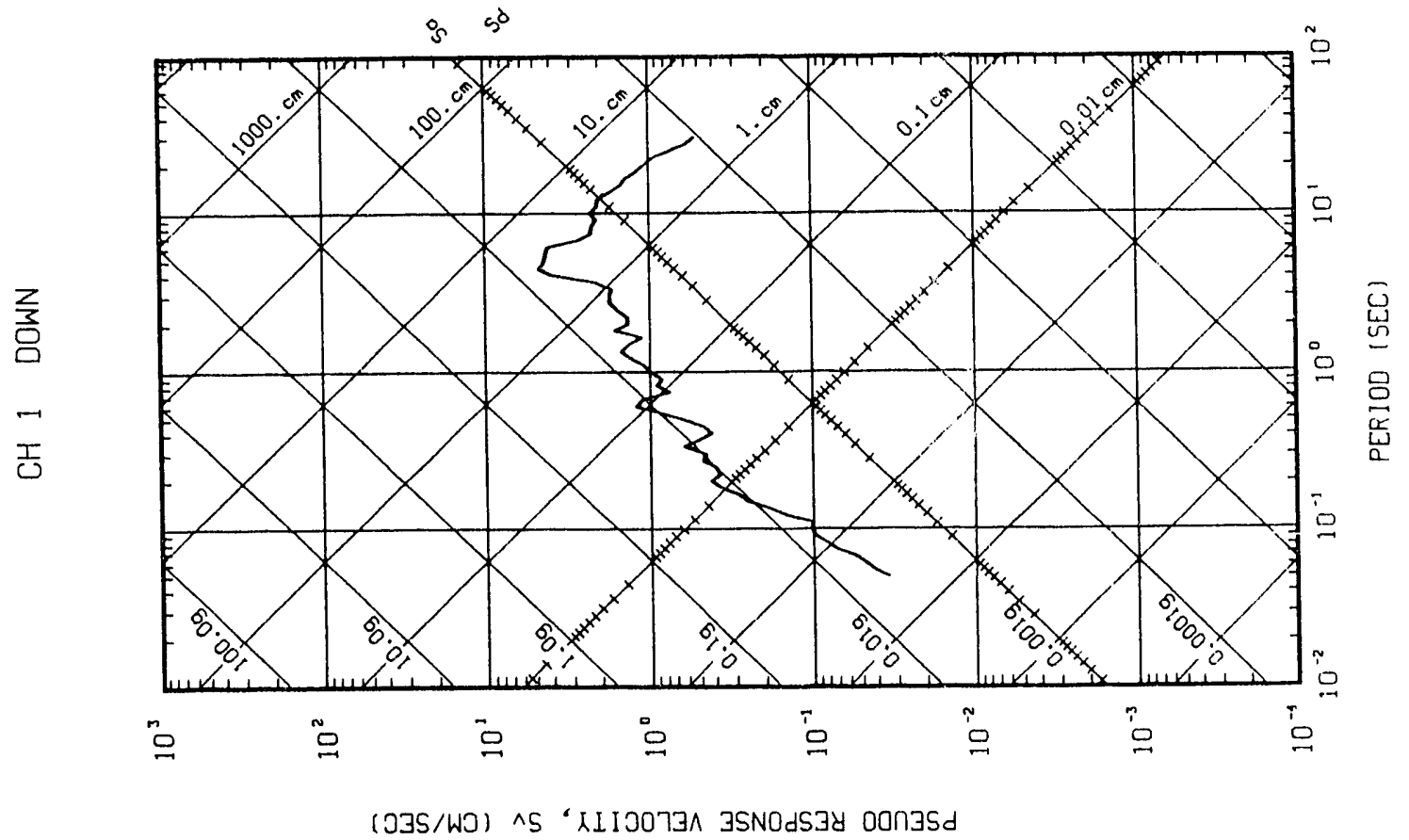
-

N

-

$r$

운

-

-

$\frac{Z}{1}$

-

e 


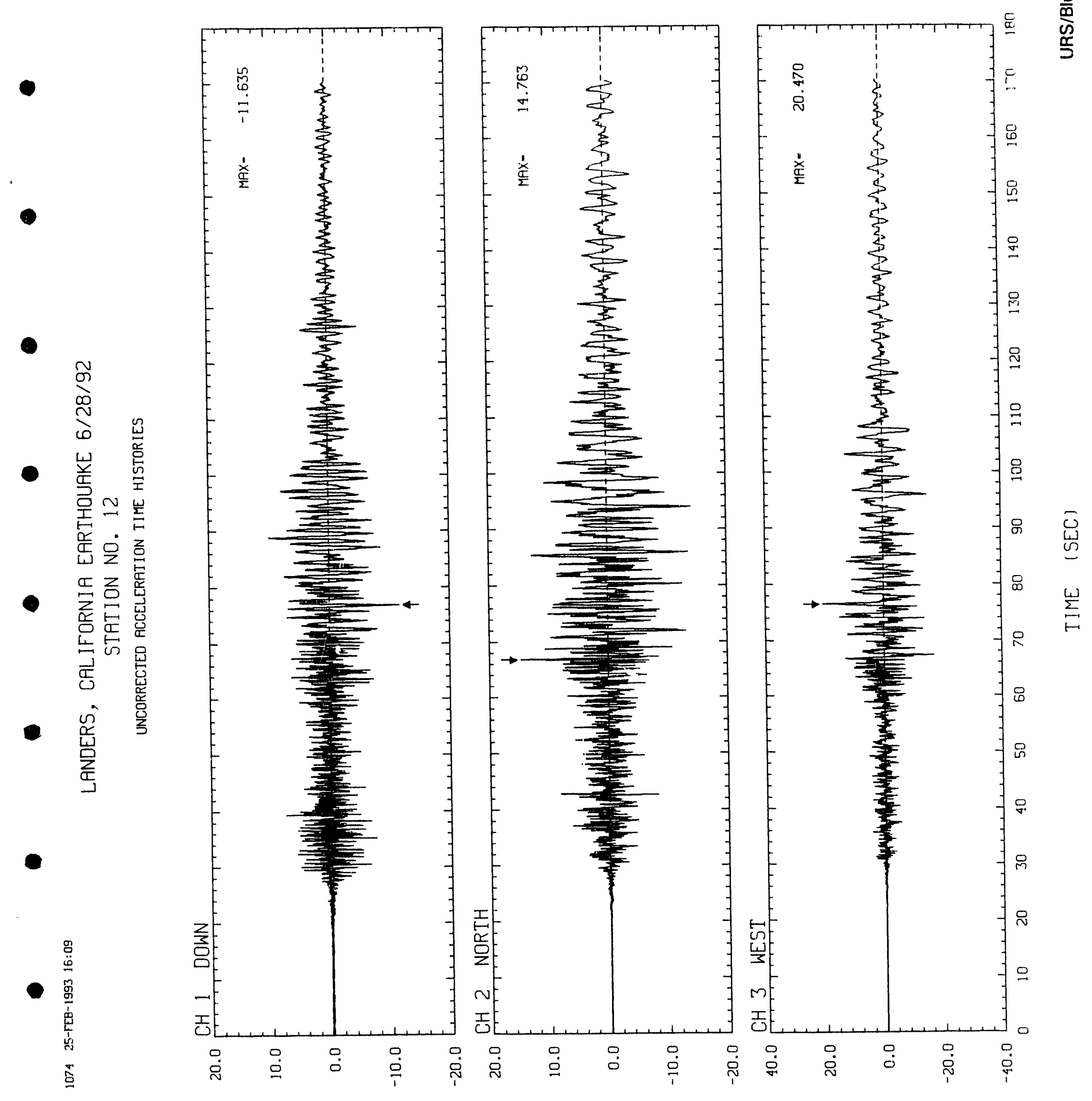




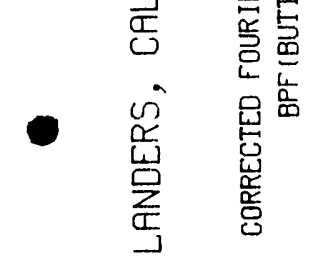

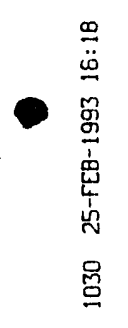
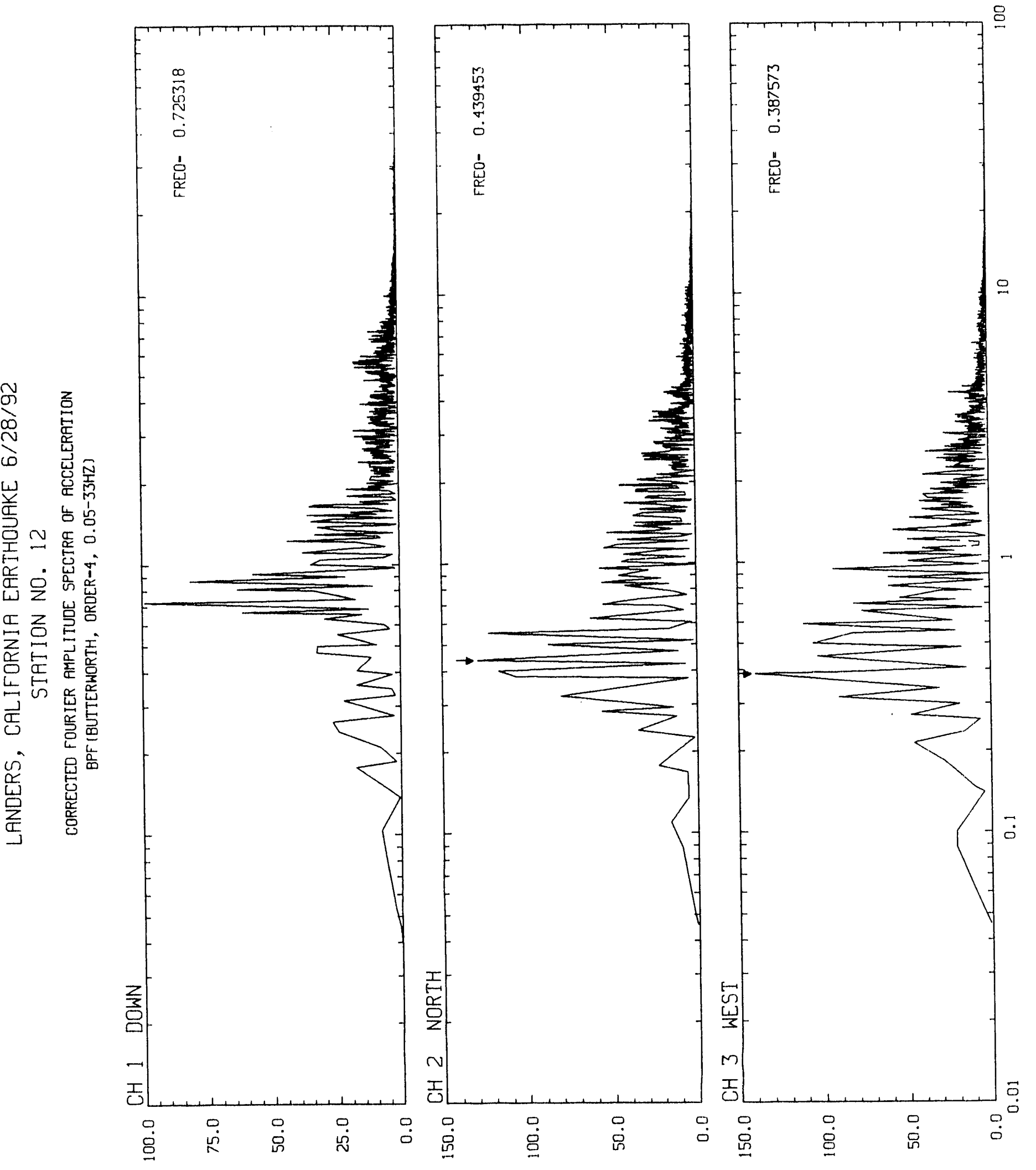

至

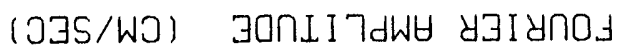




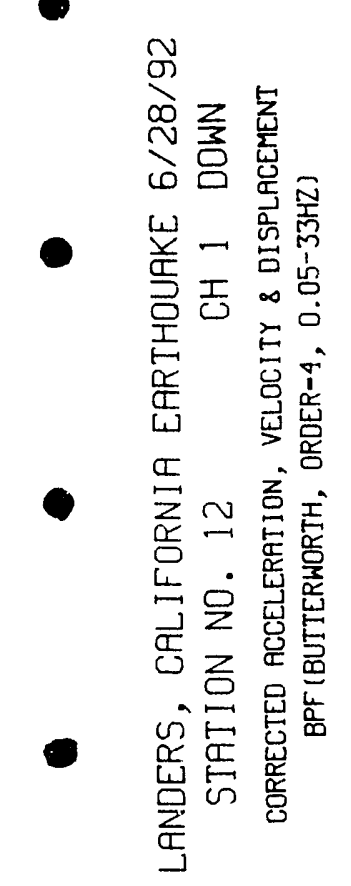

•

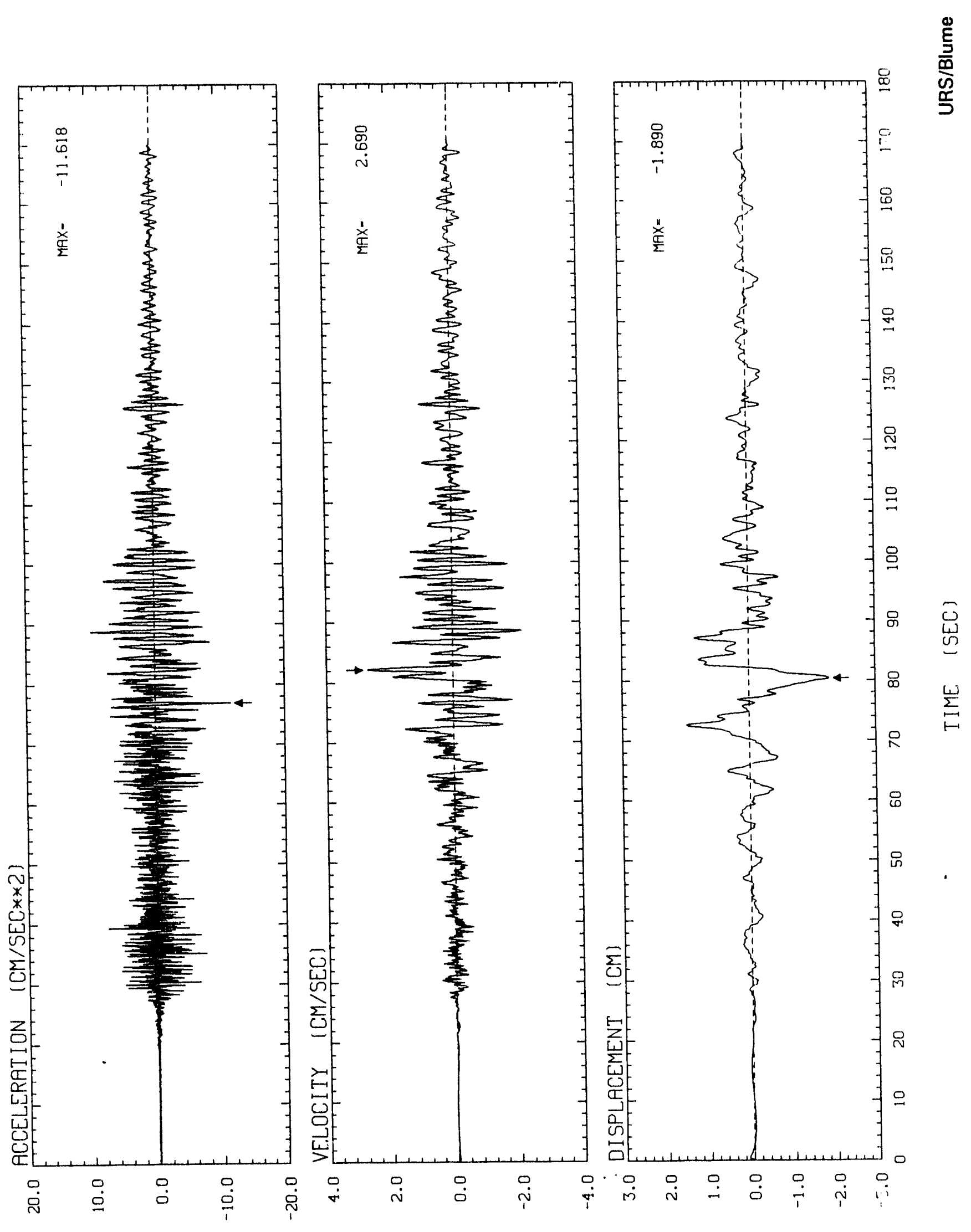




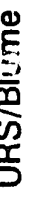

\%ั

品志

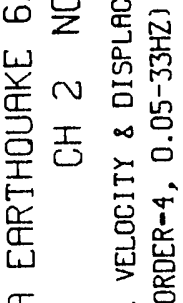

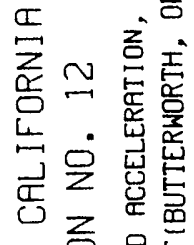

을 呢

足焉

岸点喜
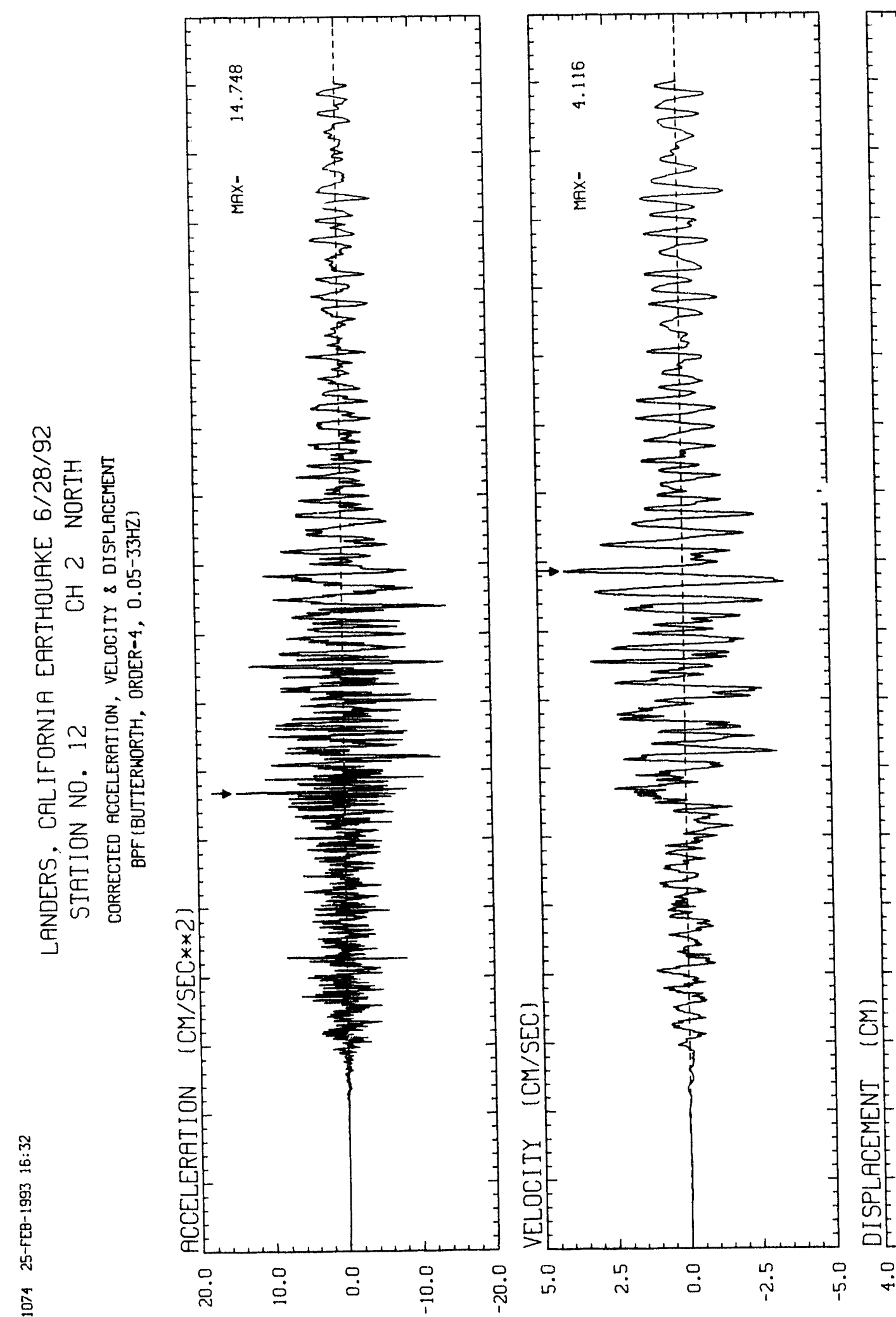

$\frac{\sqrt[n]{x}}{\frac{1}{2}}$

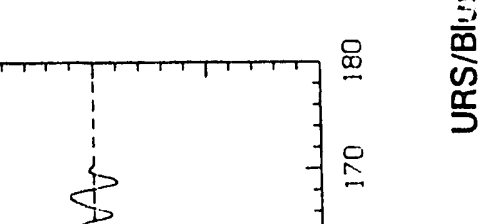

옥

욤

-

- 음

모

음

음

岕

$\sum_{i=1}^{W}$

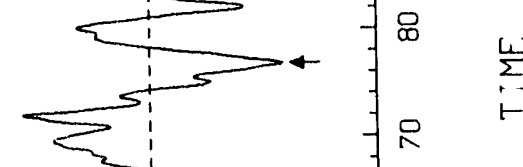

8

in

운
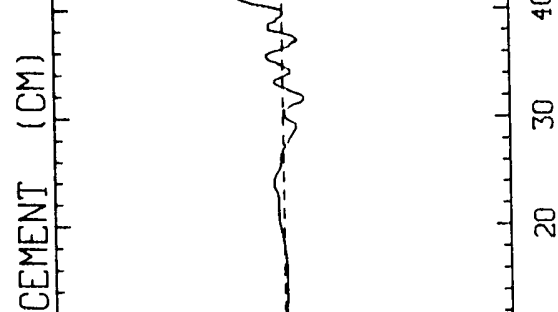

10

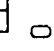

i 


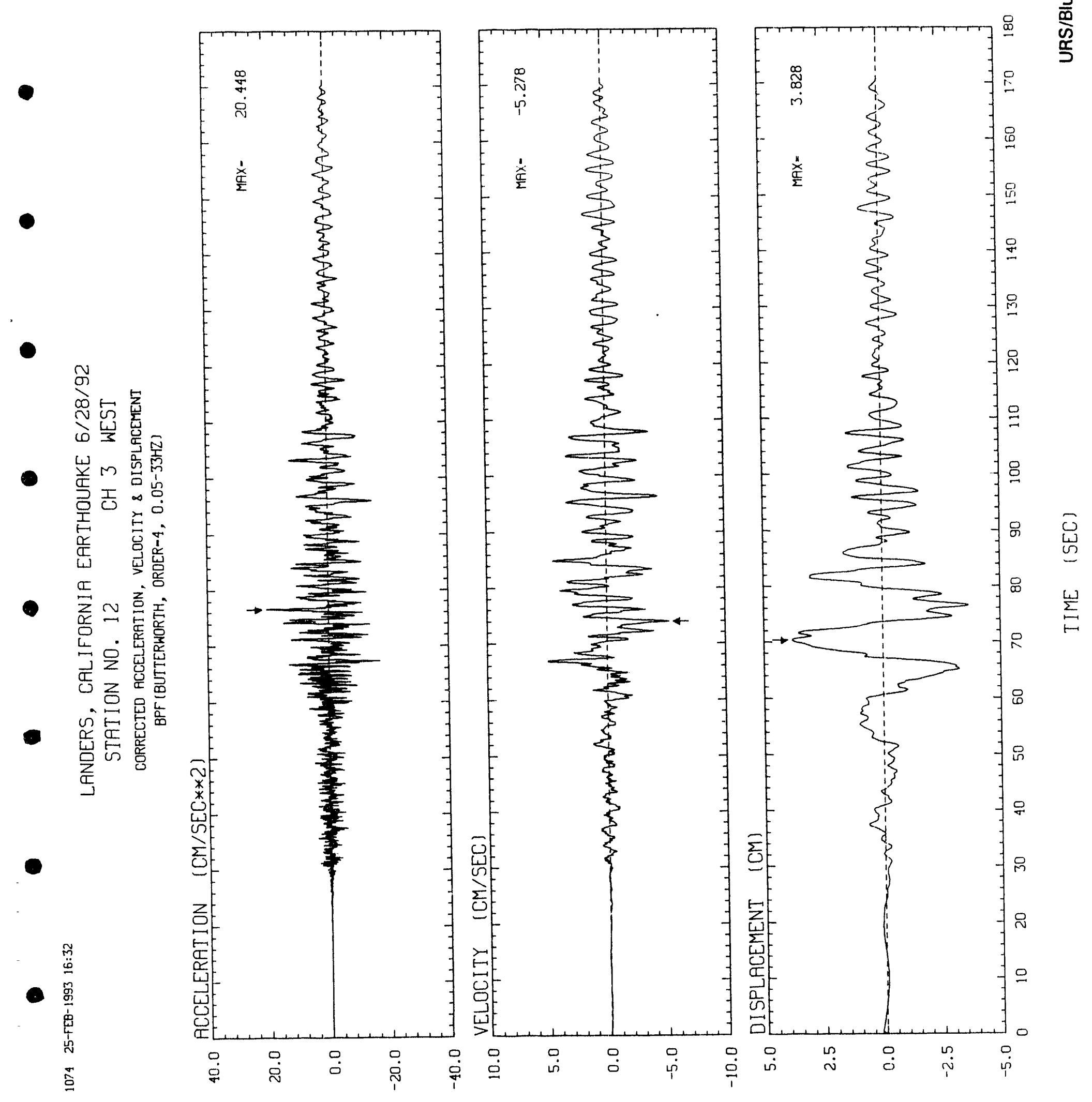

30กI I าdW 


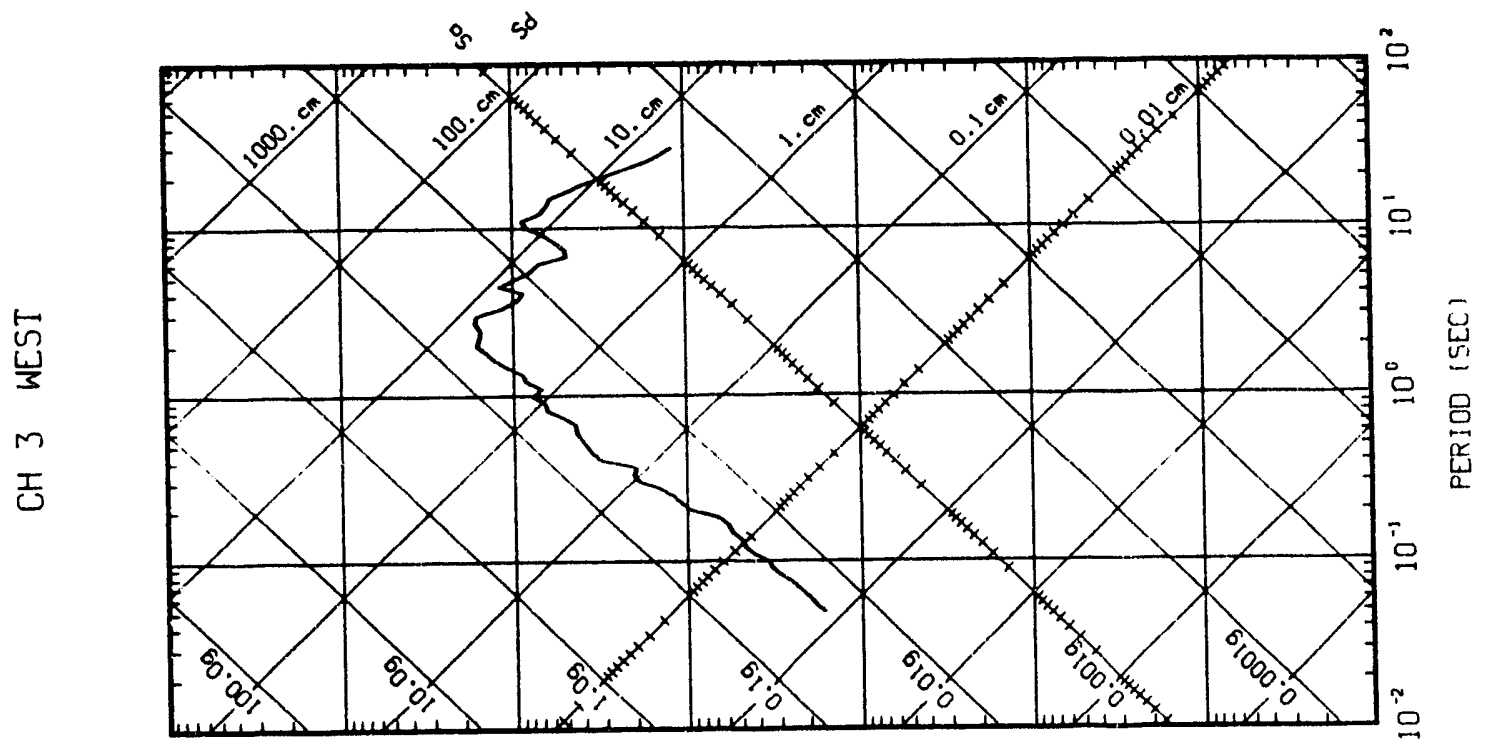

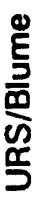
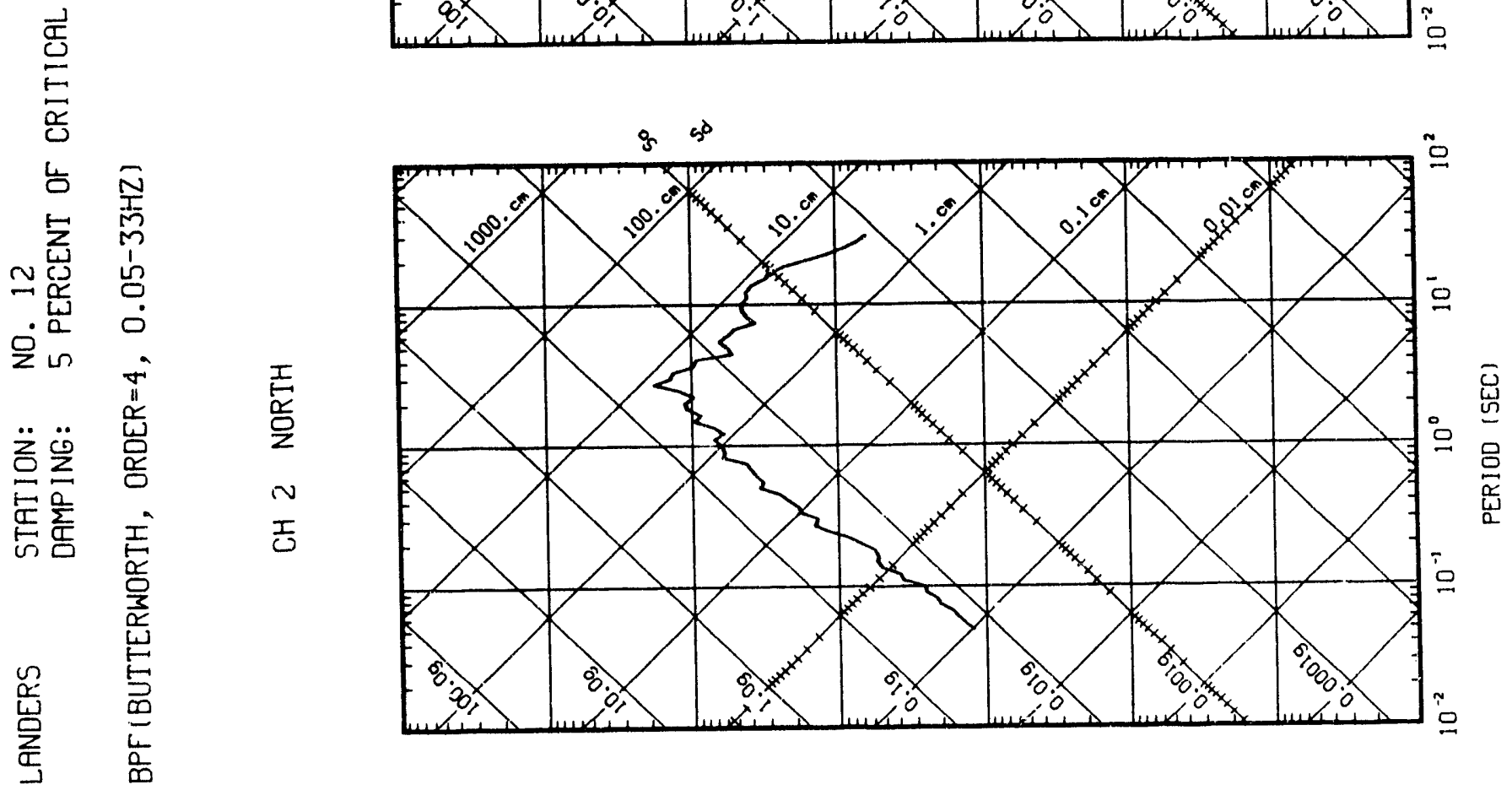

莣离悹

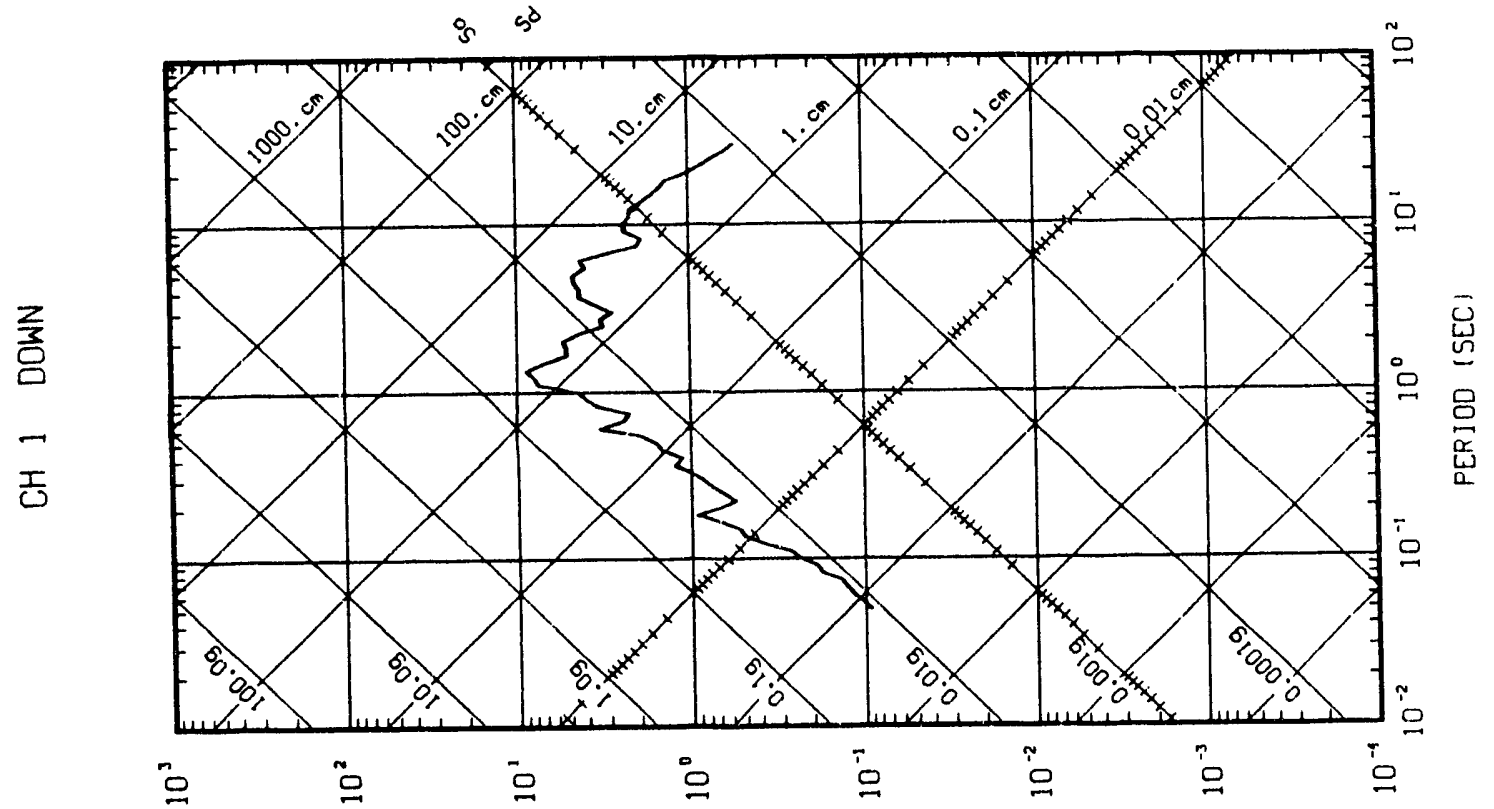

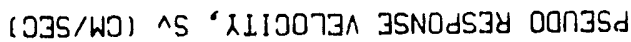


$\sum_{\infty}^{\frac{1}{6}}$

$m$

○

O 


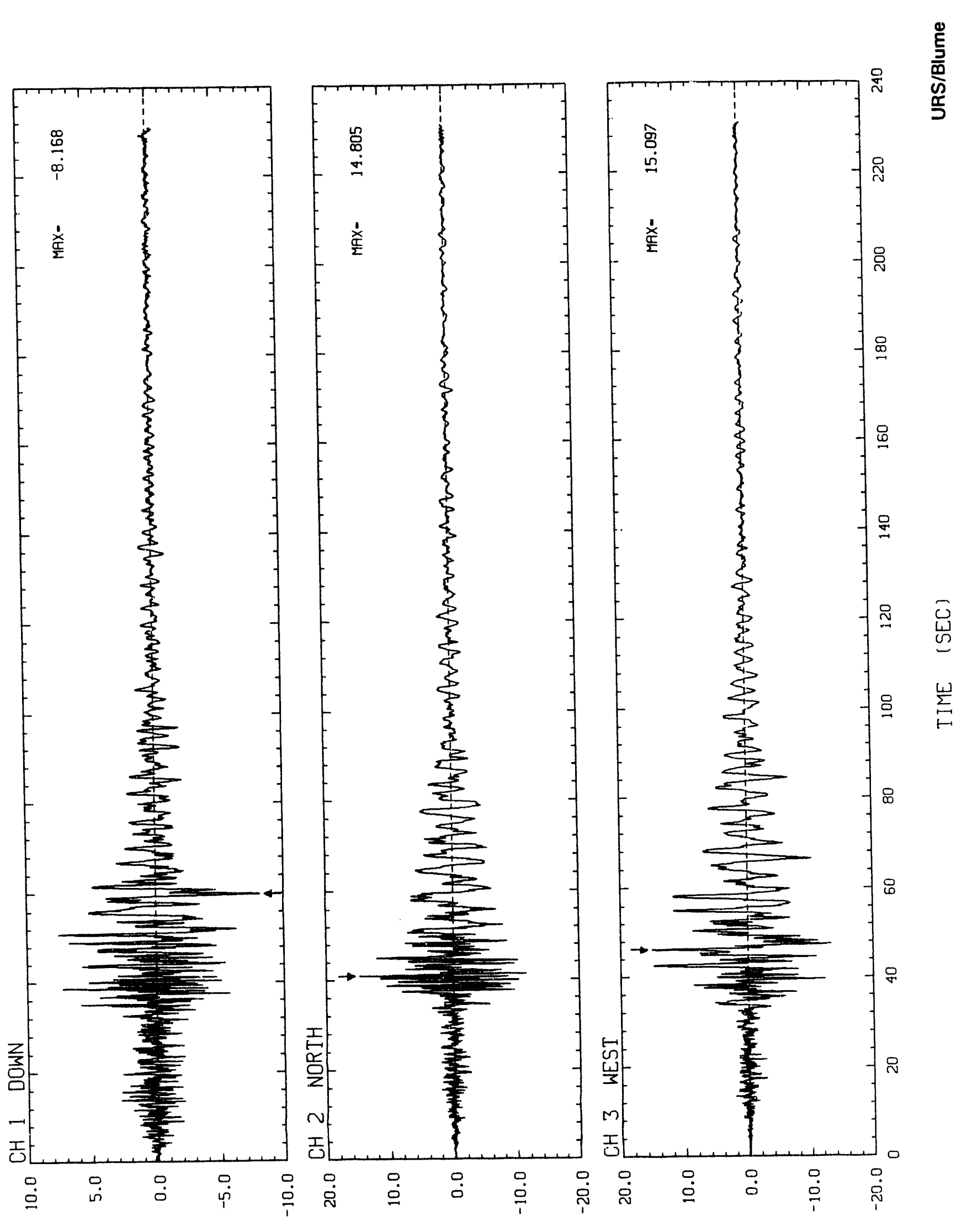

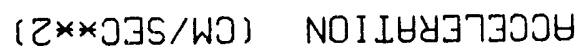




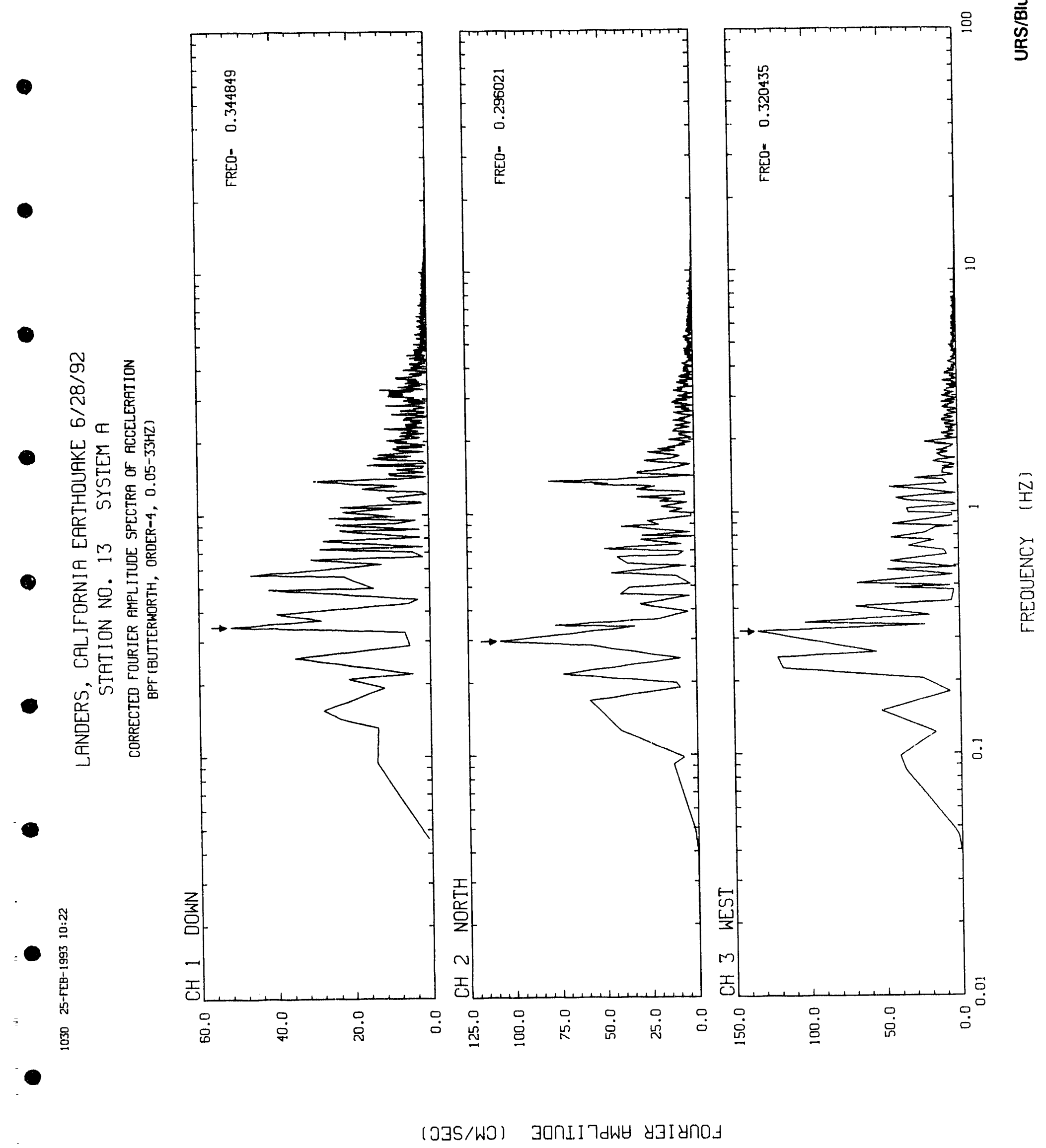




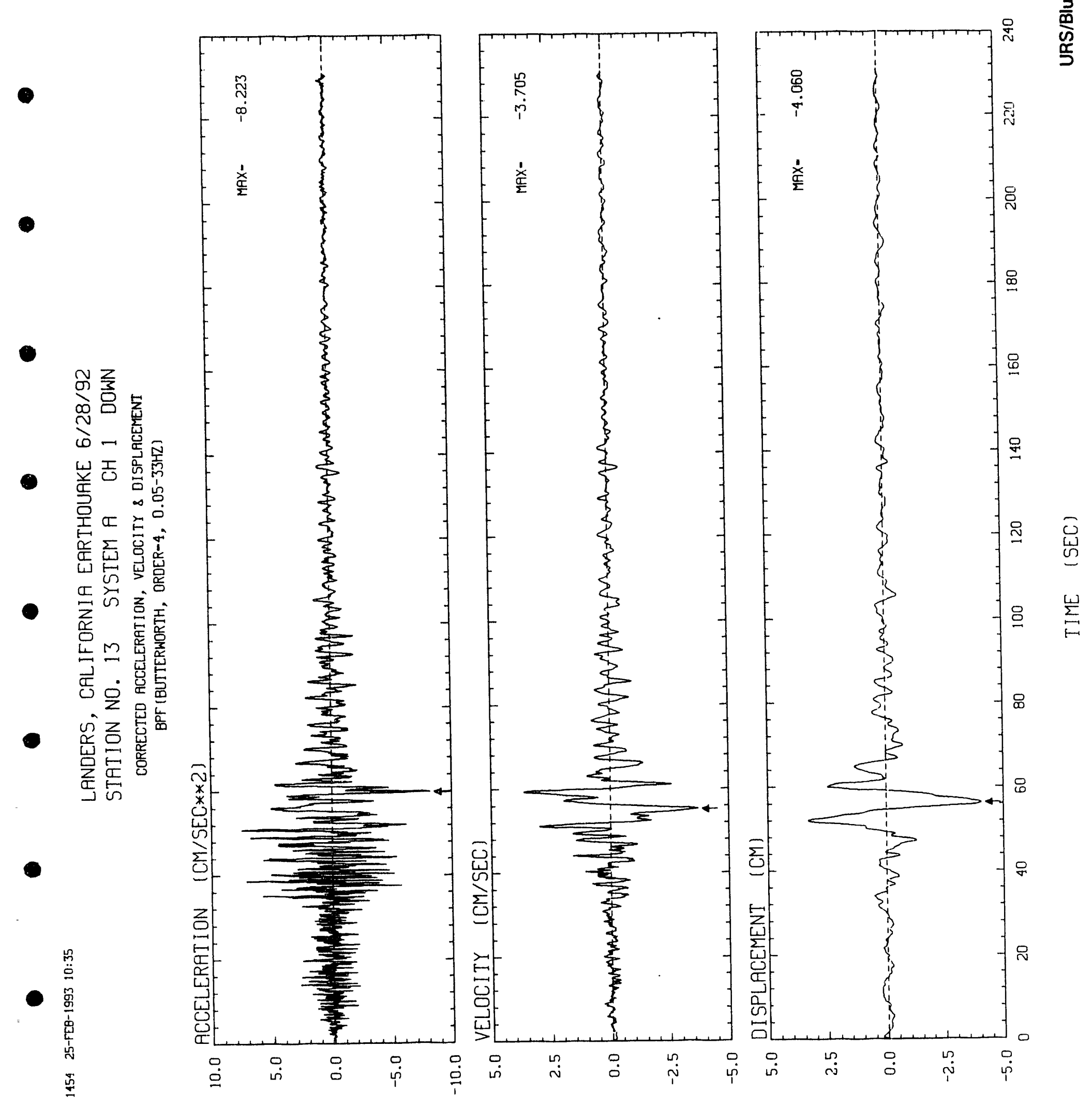

JanI I 7dWH 


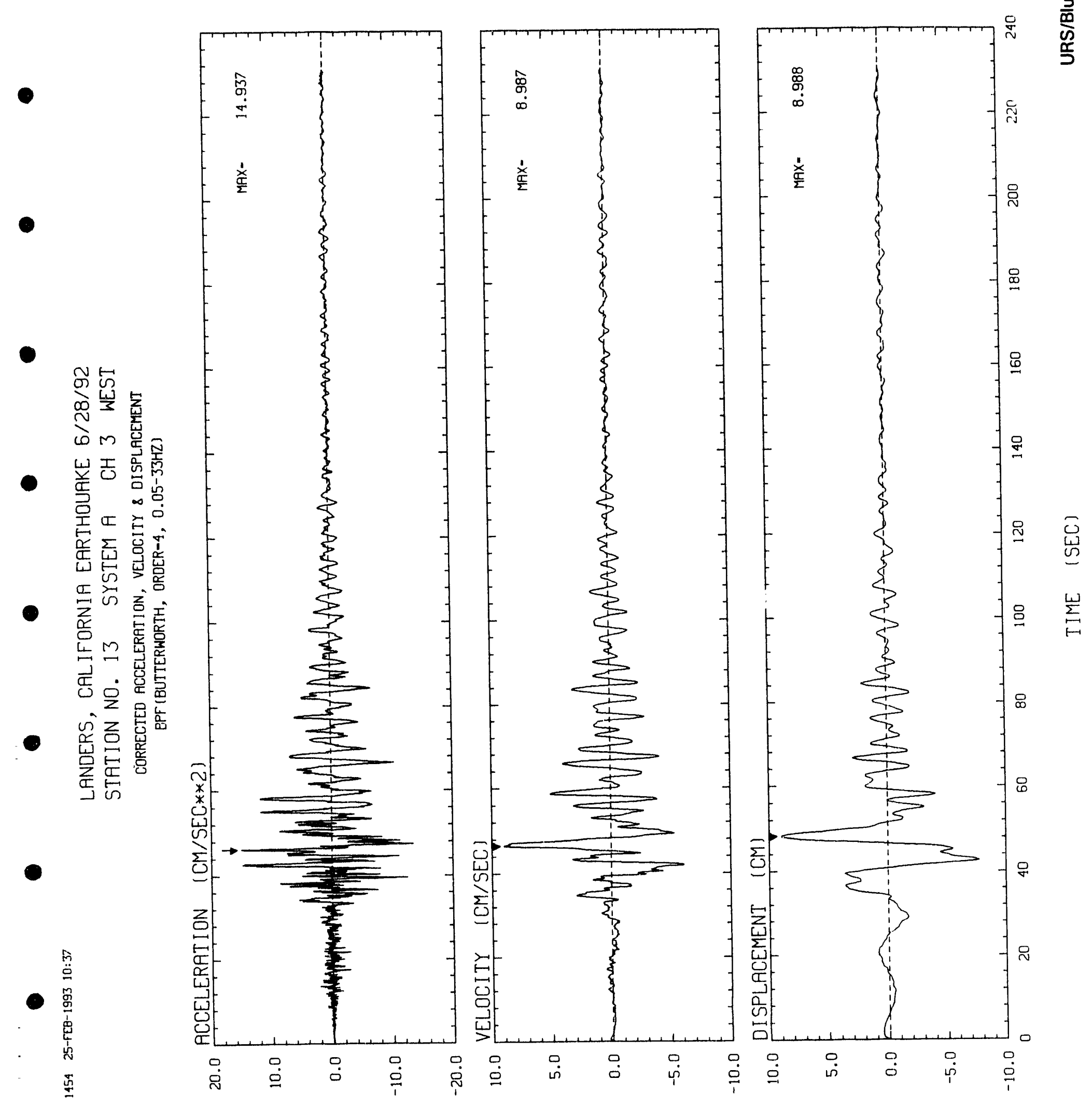

JOกI I 7dWH 

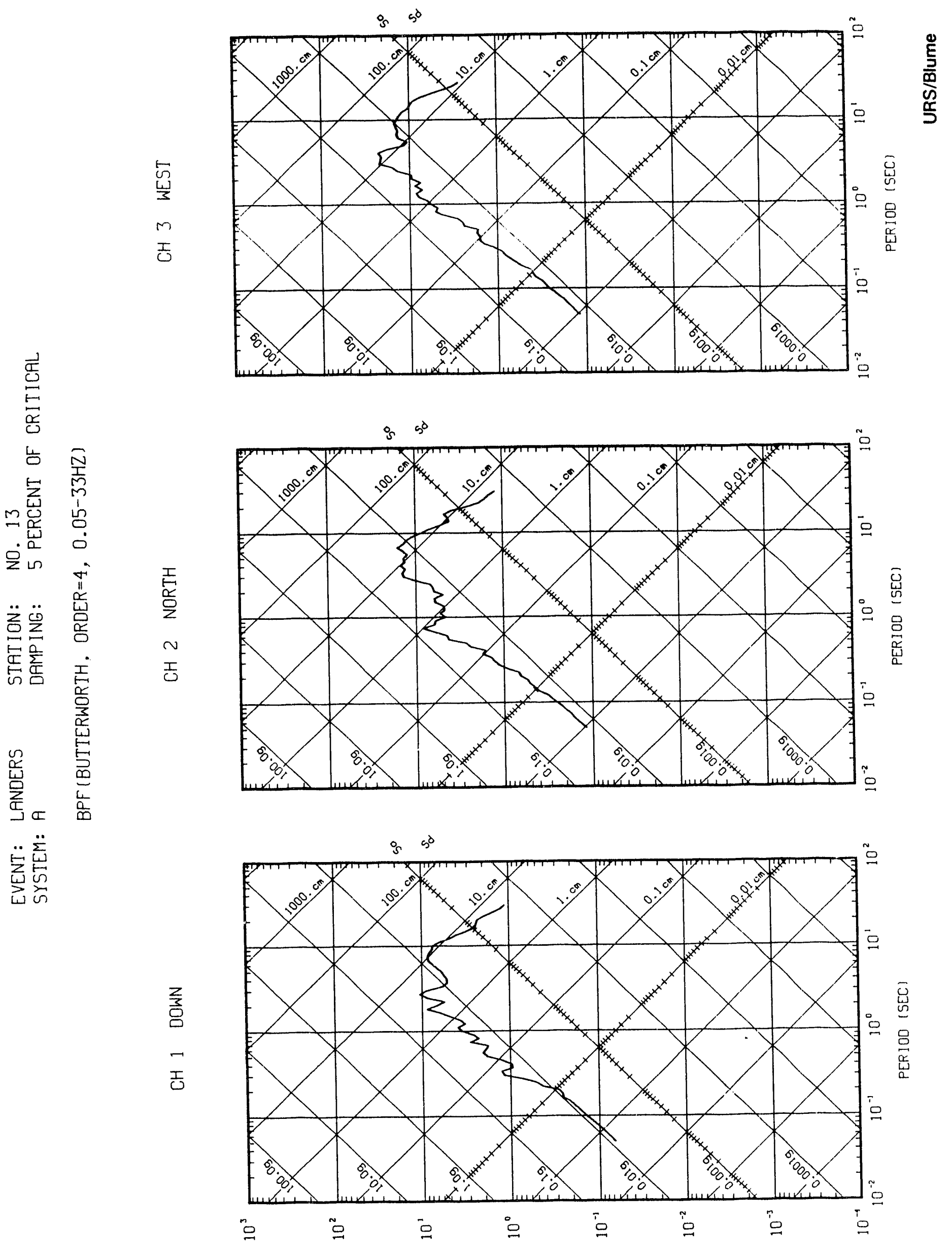

(OJS/WO) ^S ' XIIJOTJA JSNOdS3Y OONISd 
$m$

$\sum_{\infty}^{\frac{5}{\omega}}$

$m$

$r$

i

-

○

- 


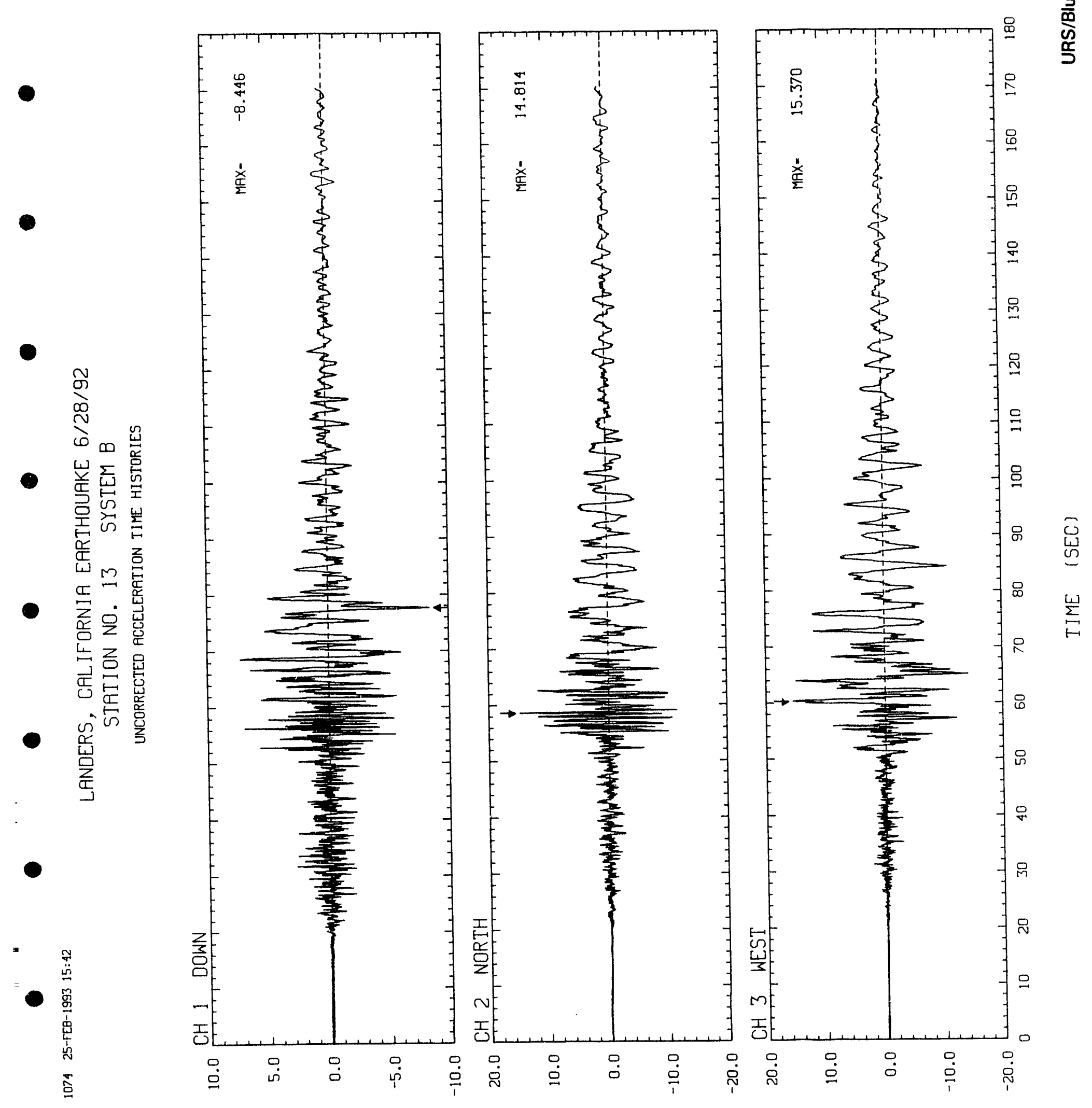




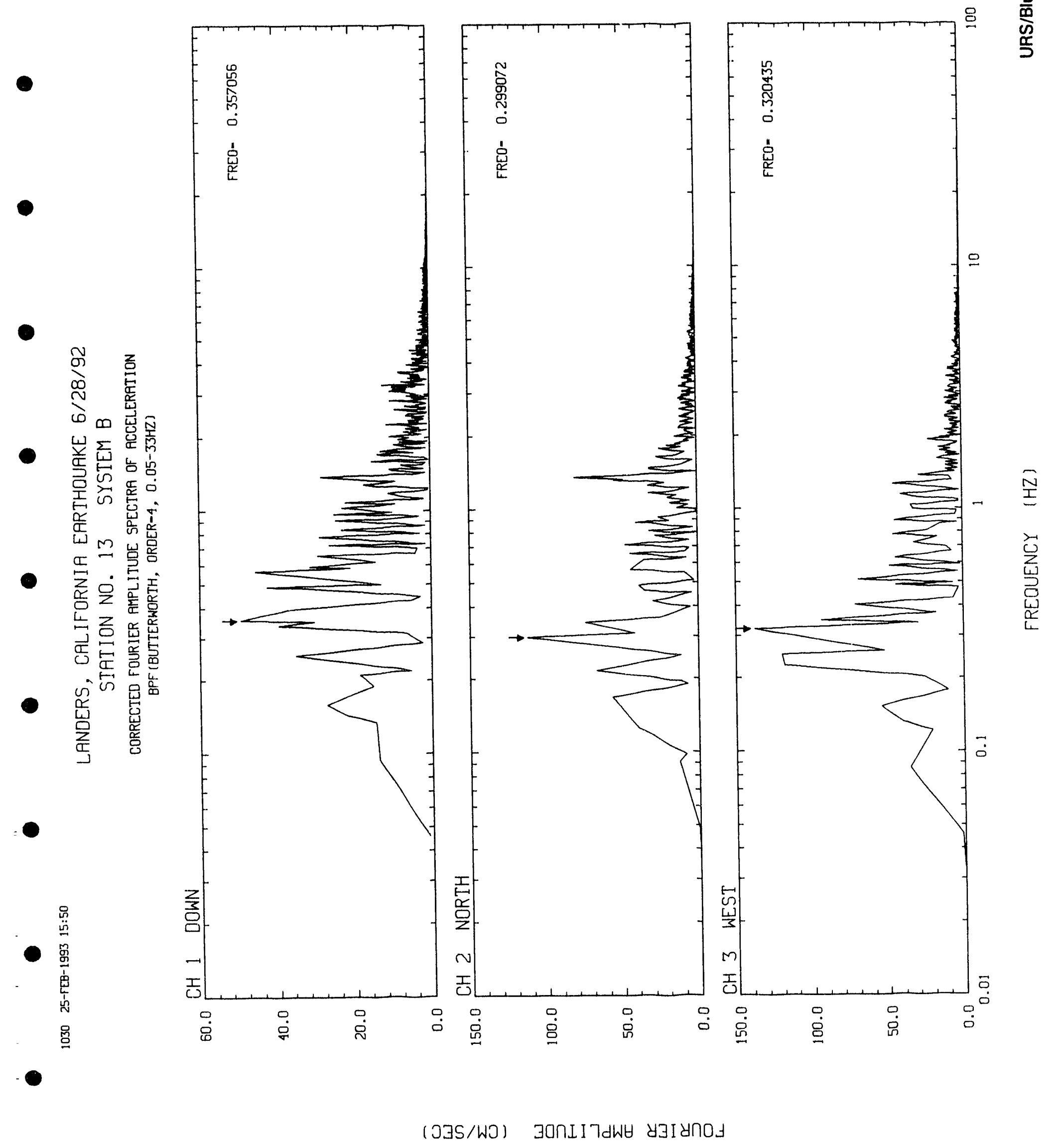




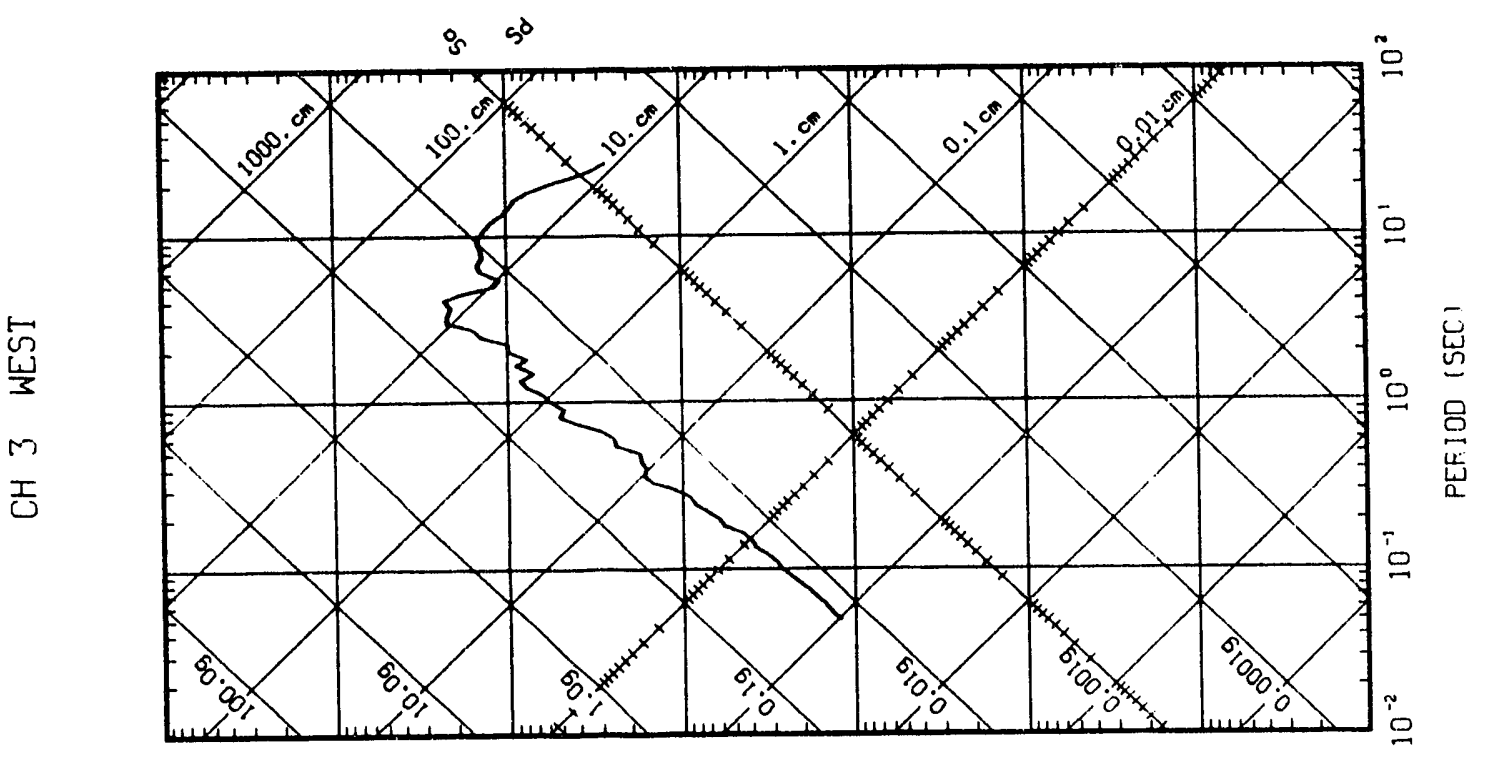

总
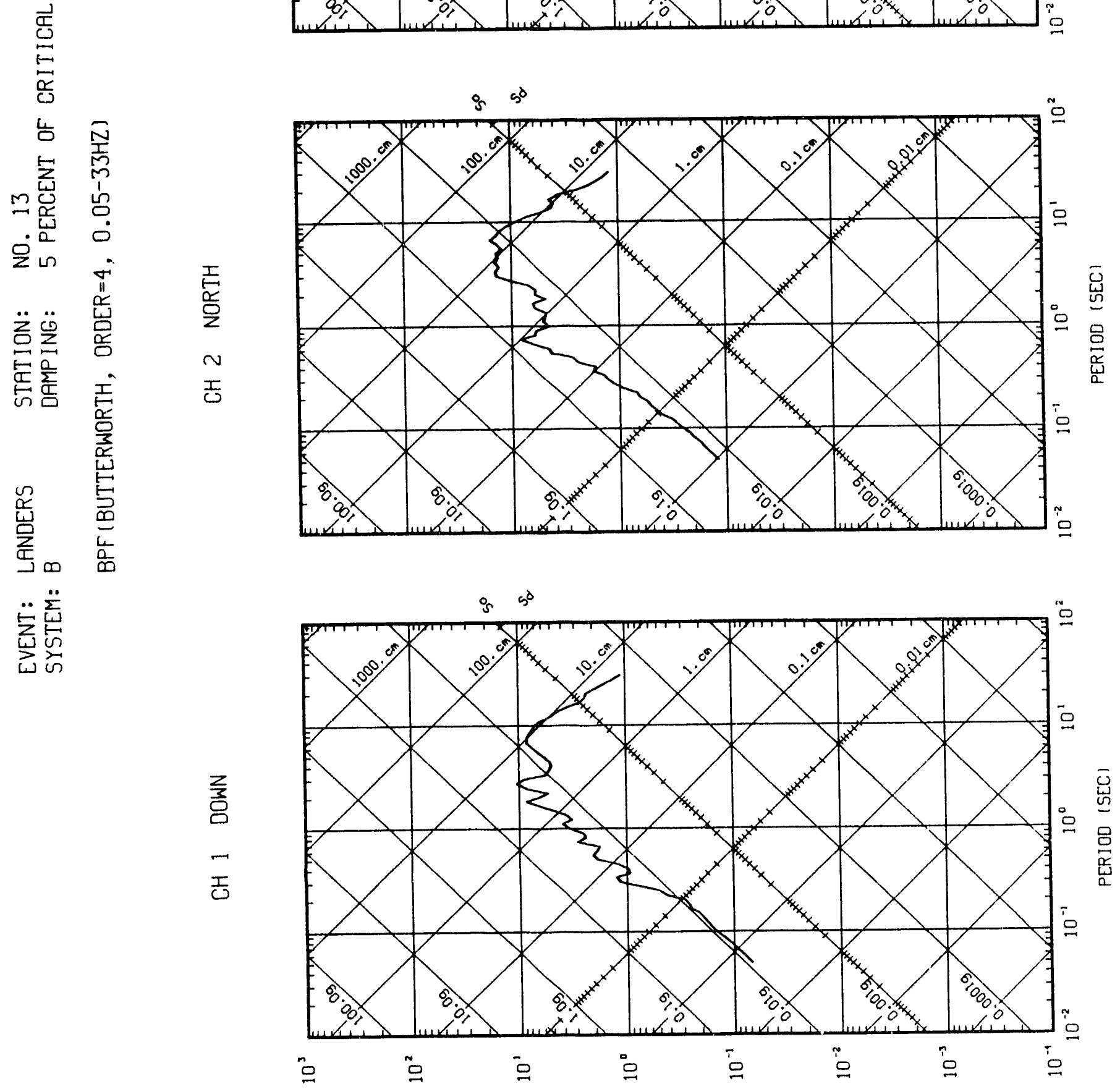

(OJS/WO) ^S 'XIIJOT3^ 3SNOdS3Y OON3SA 
$\bullet$

$\bullet$

-

-

$\pm$

$\stackrel{0}{Z}$

$\bullet$

-

•

-

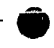




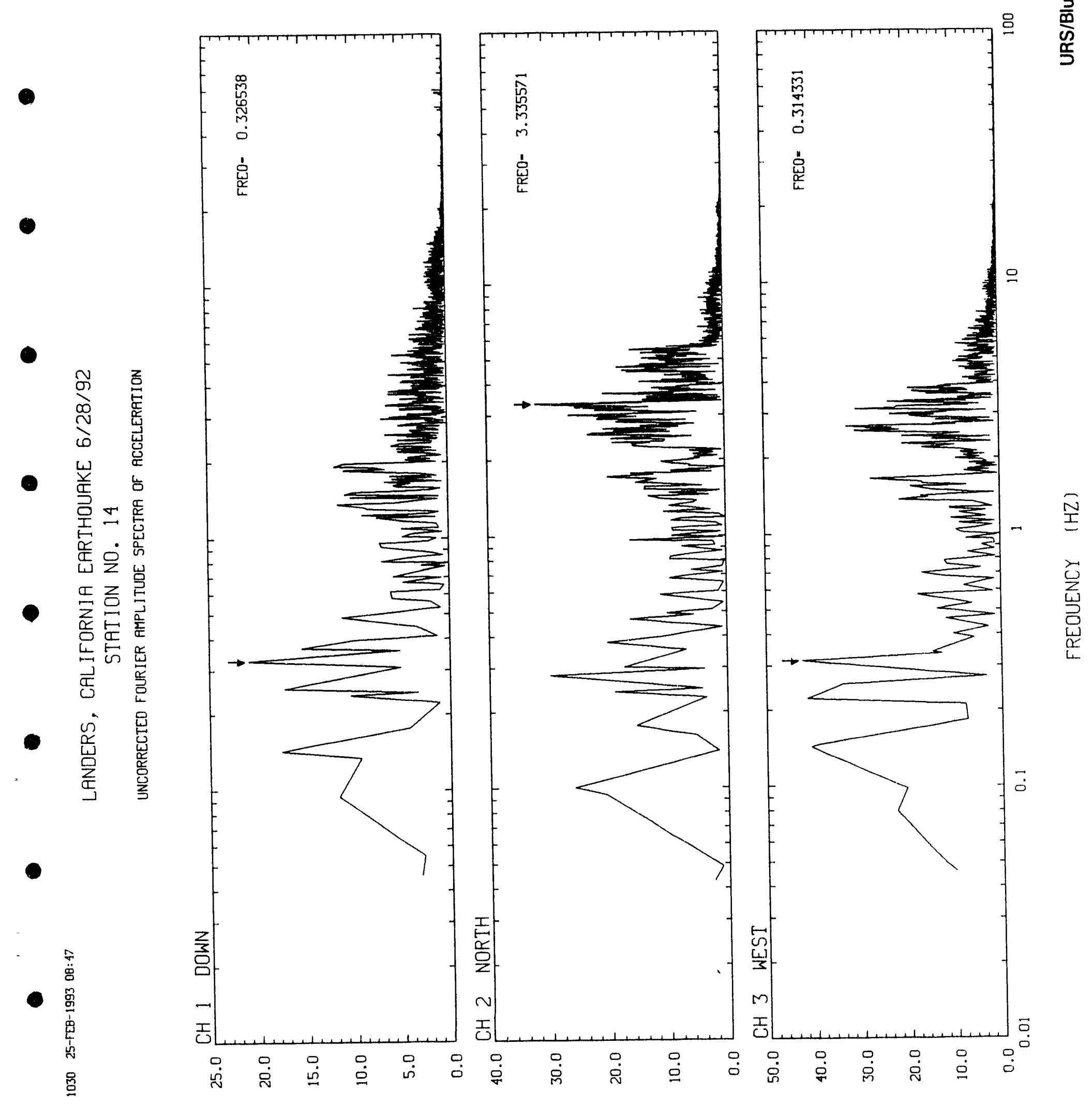

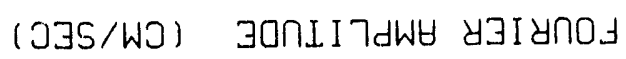




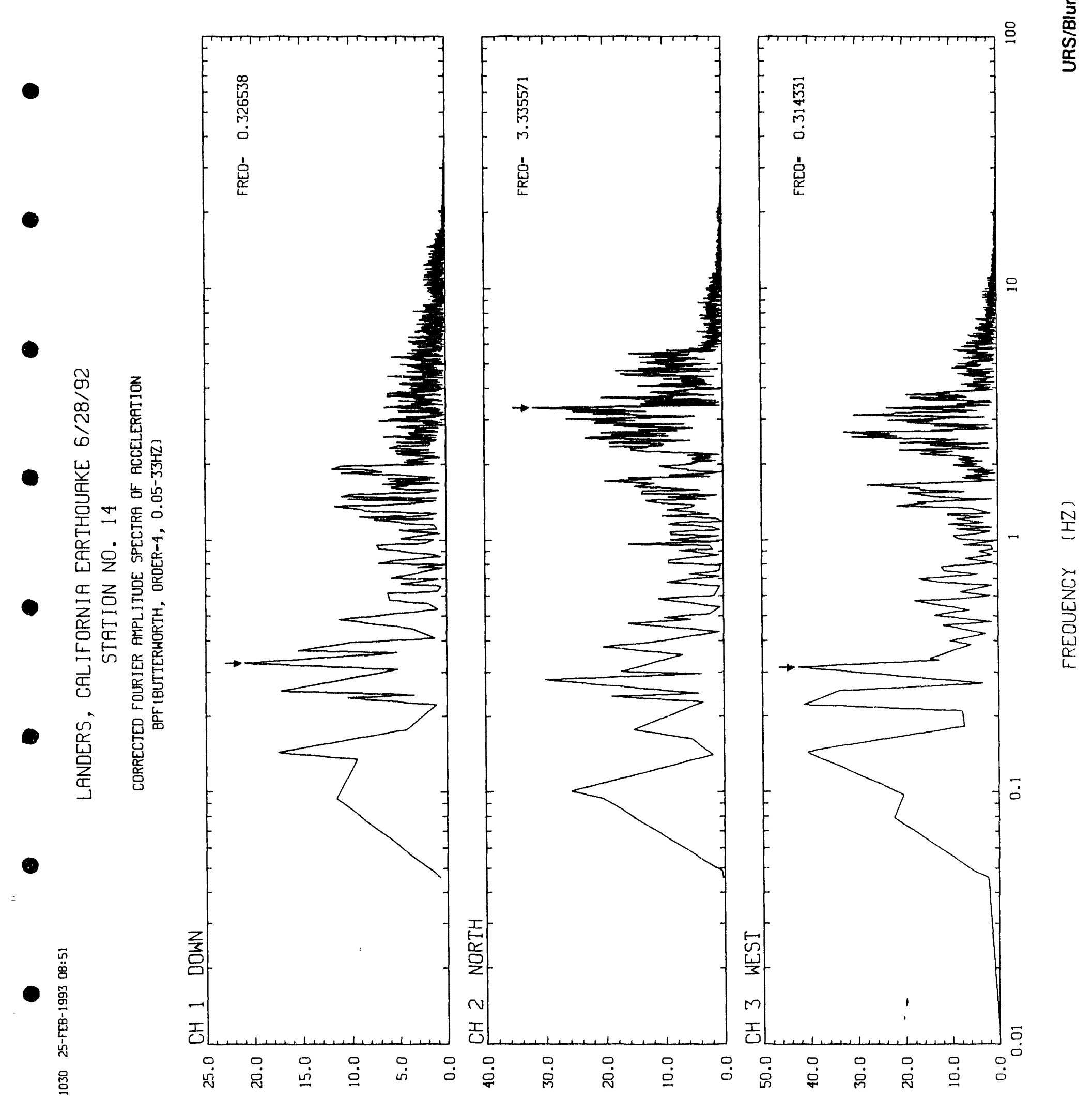

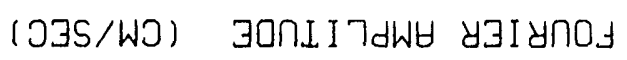




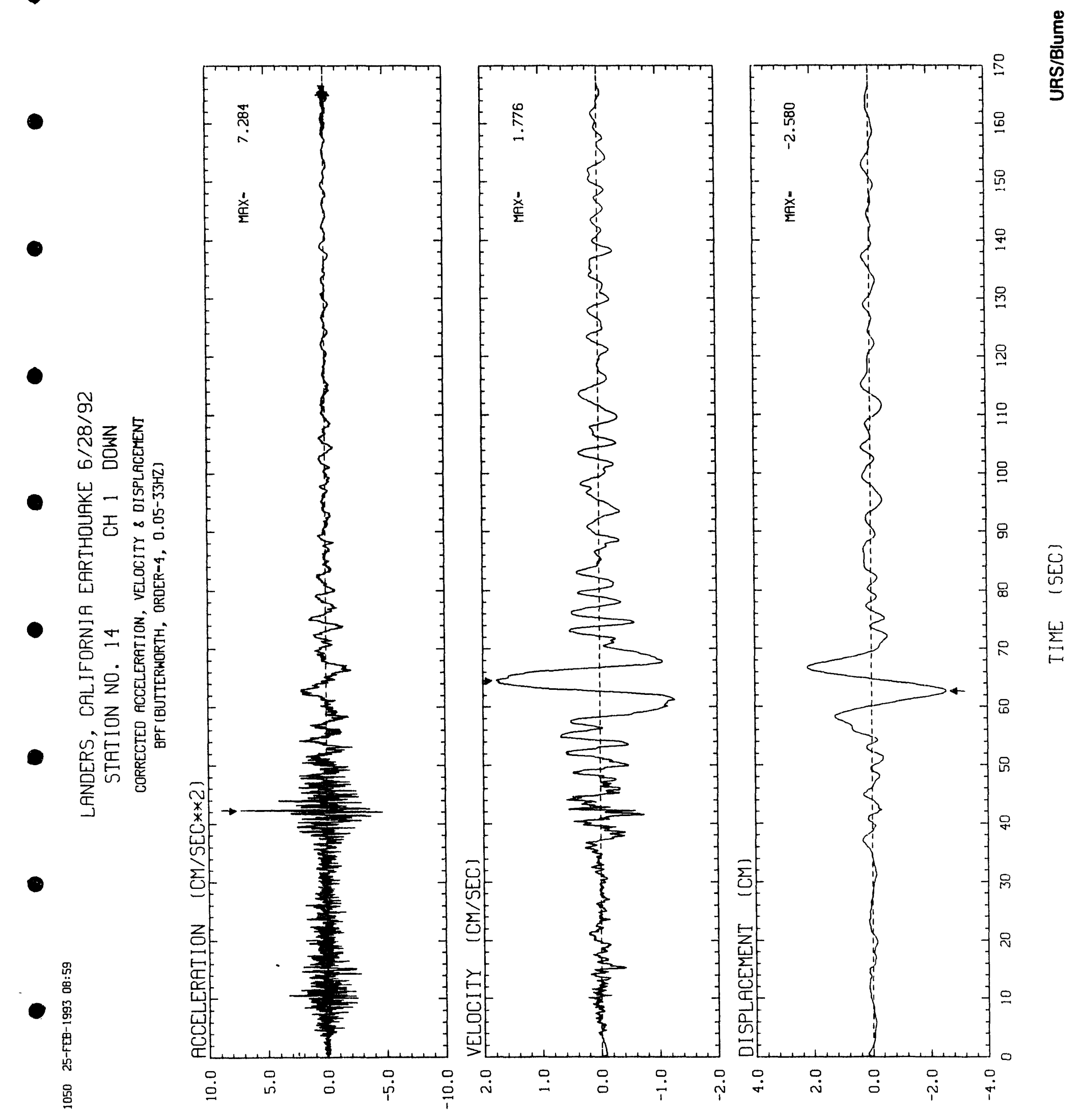

JONLI I TWU 


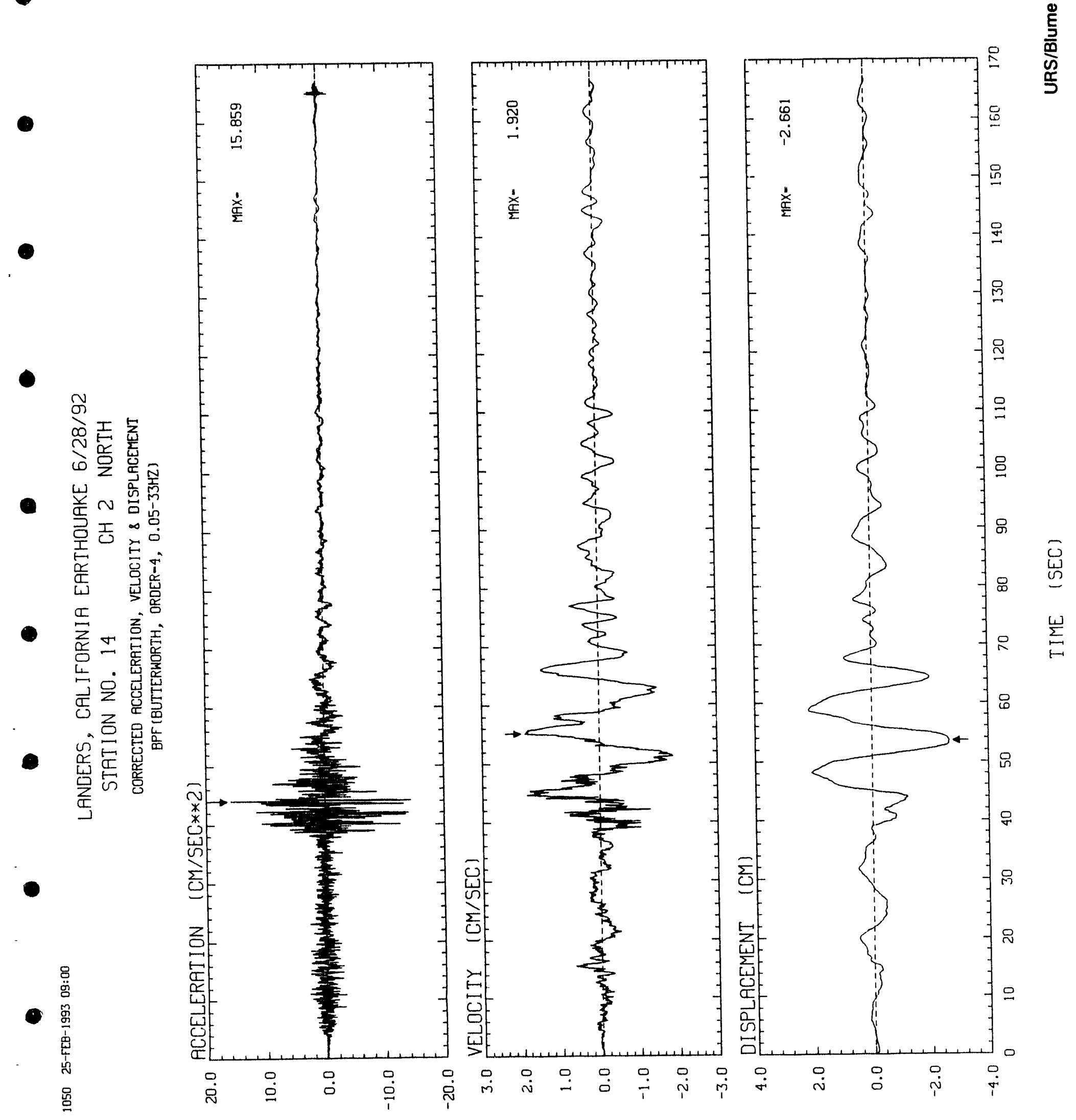




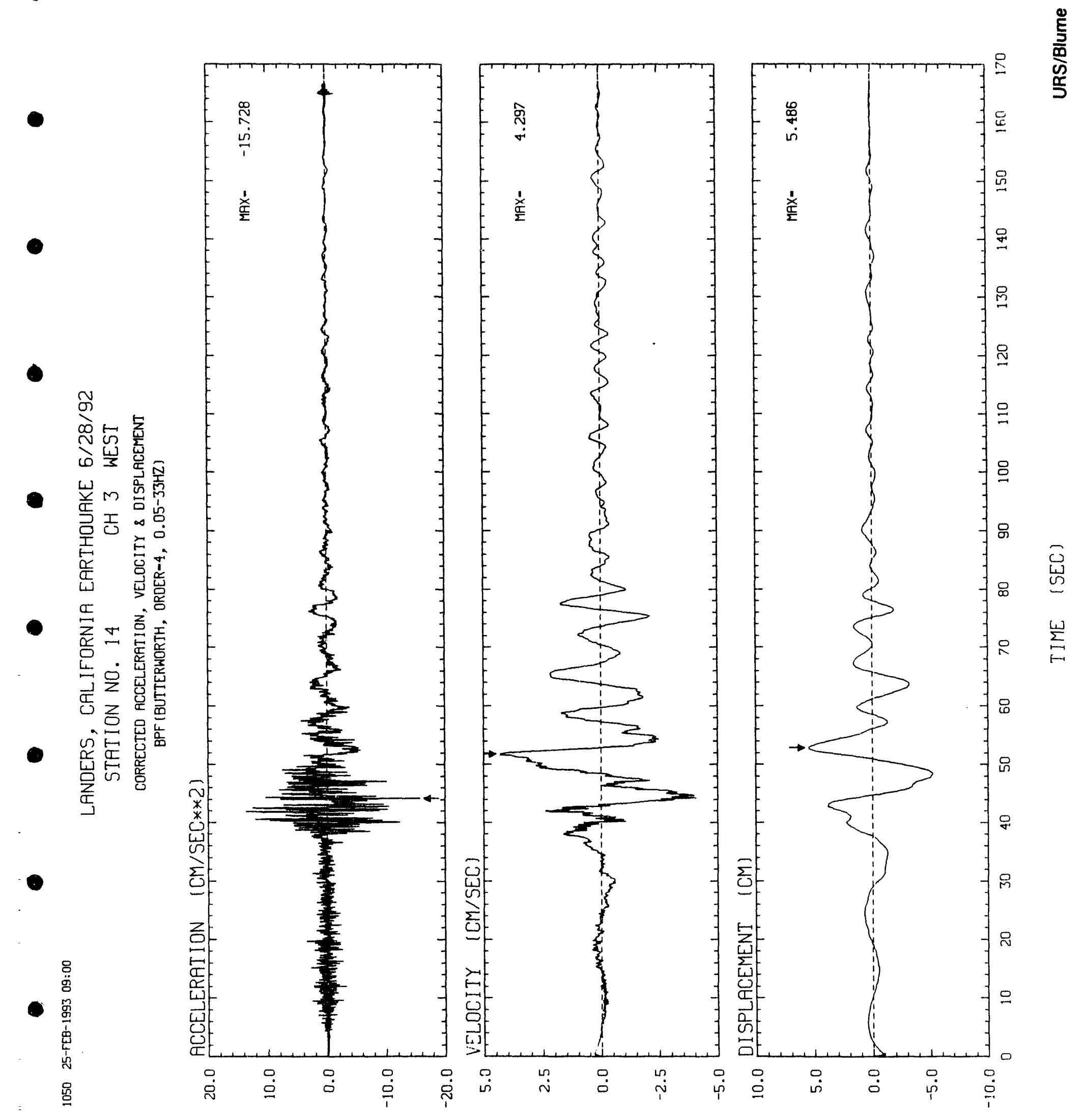

30กLI IdWH 

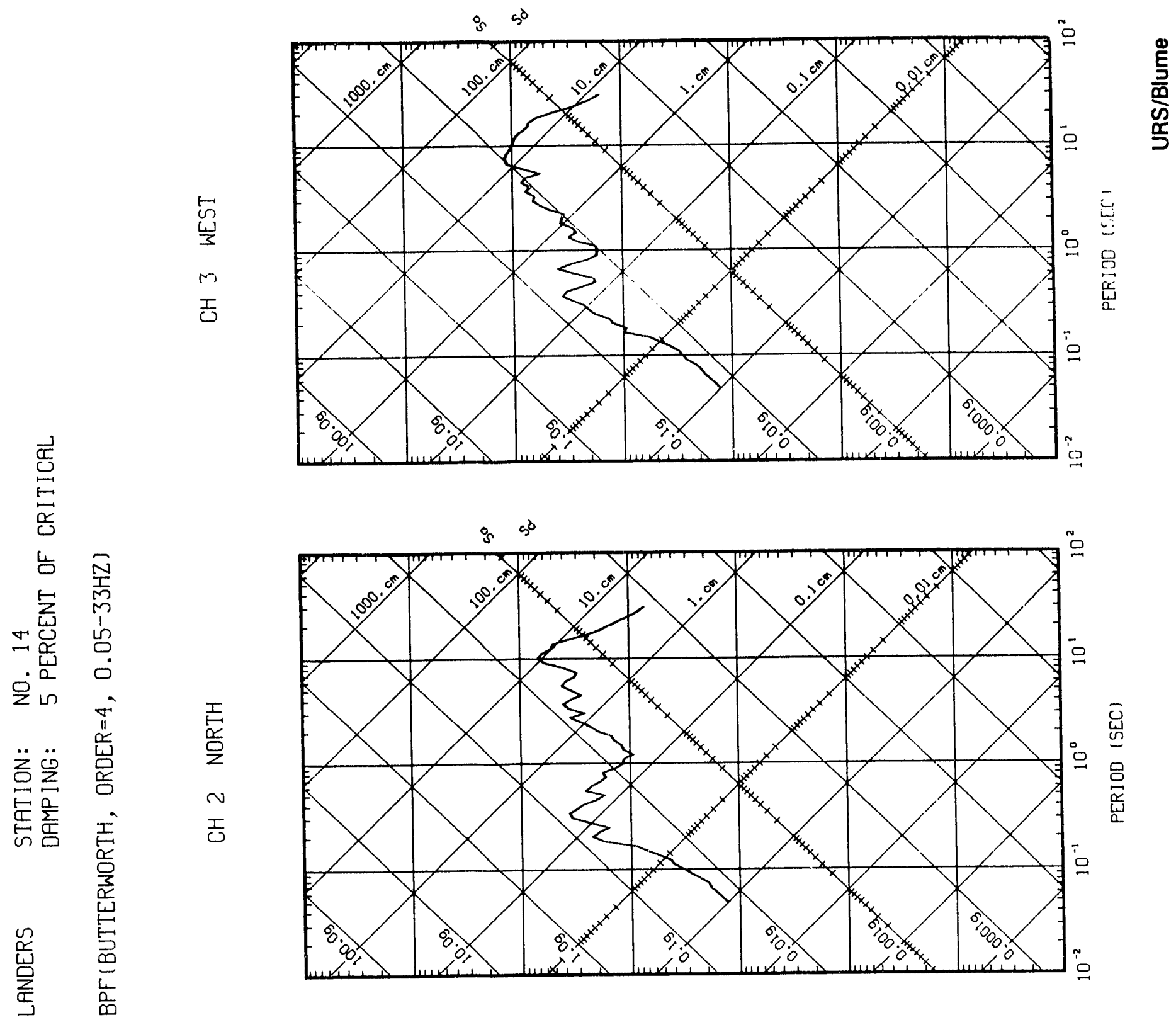

䓌悹点

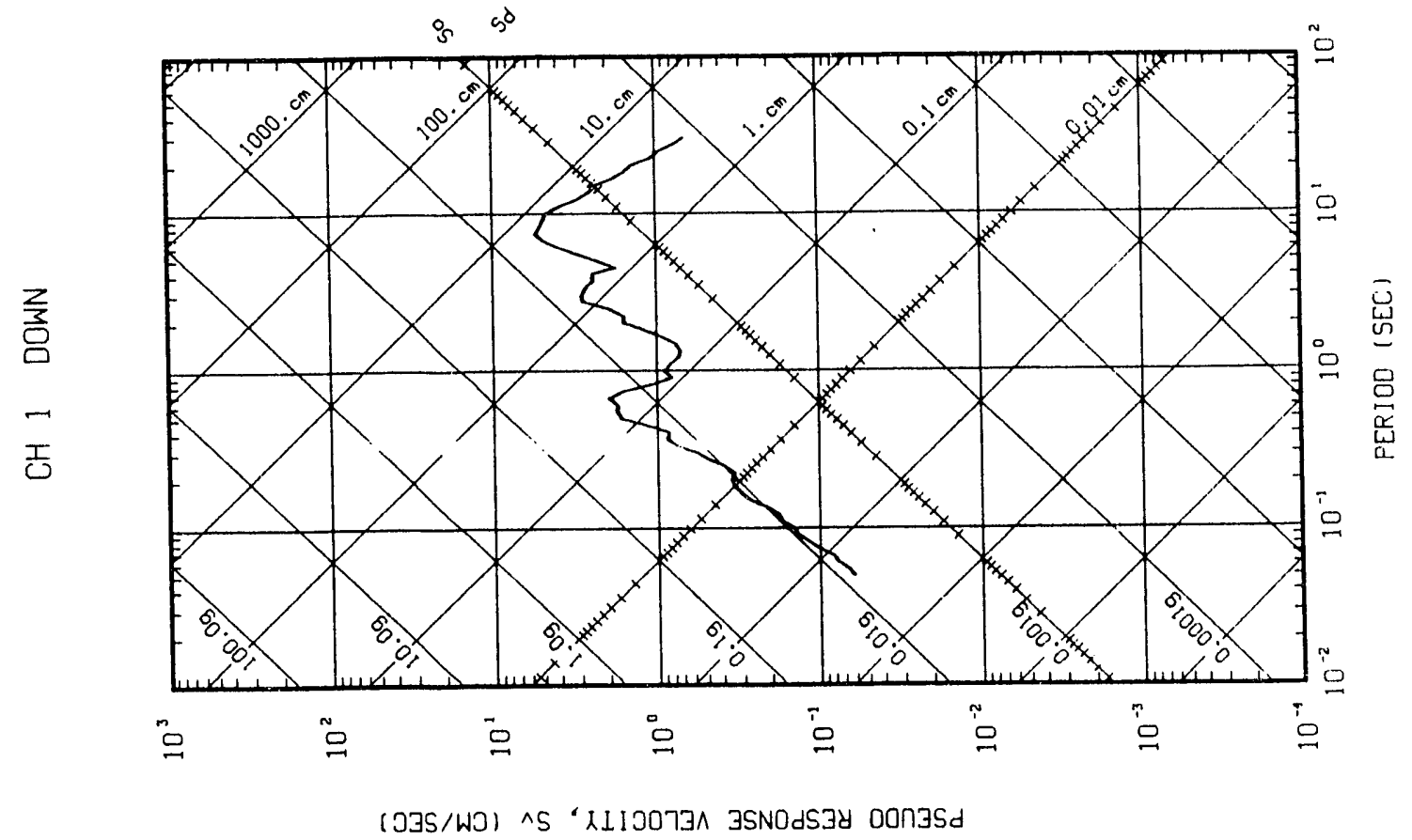


$\varangle$

$\sum_{\omega}^{\frac{\sum}{\omega}}$

움

은

O

$F$

क

○ 


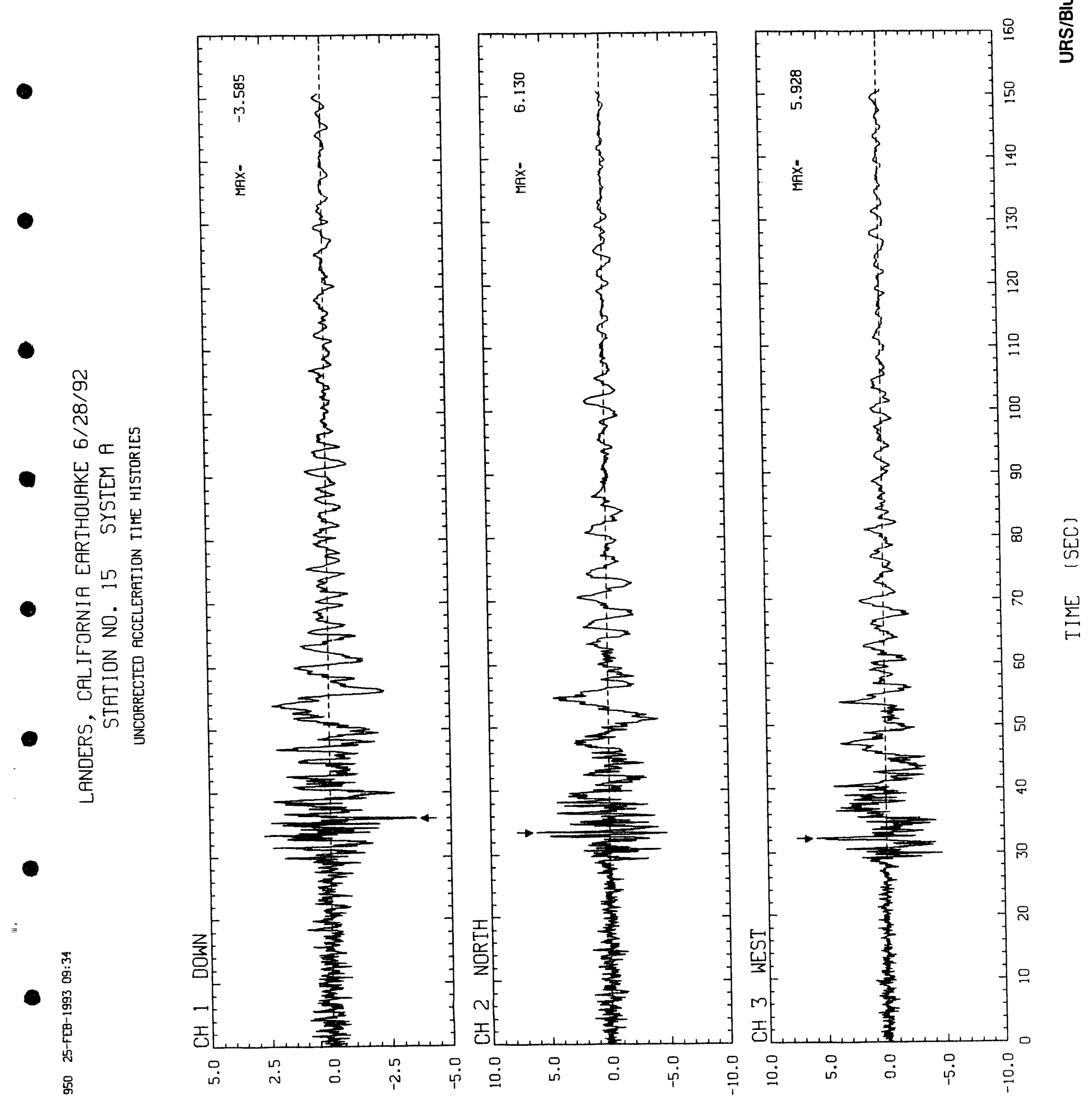

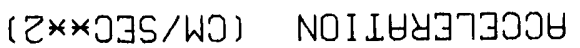




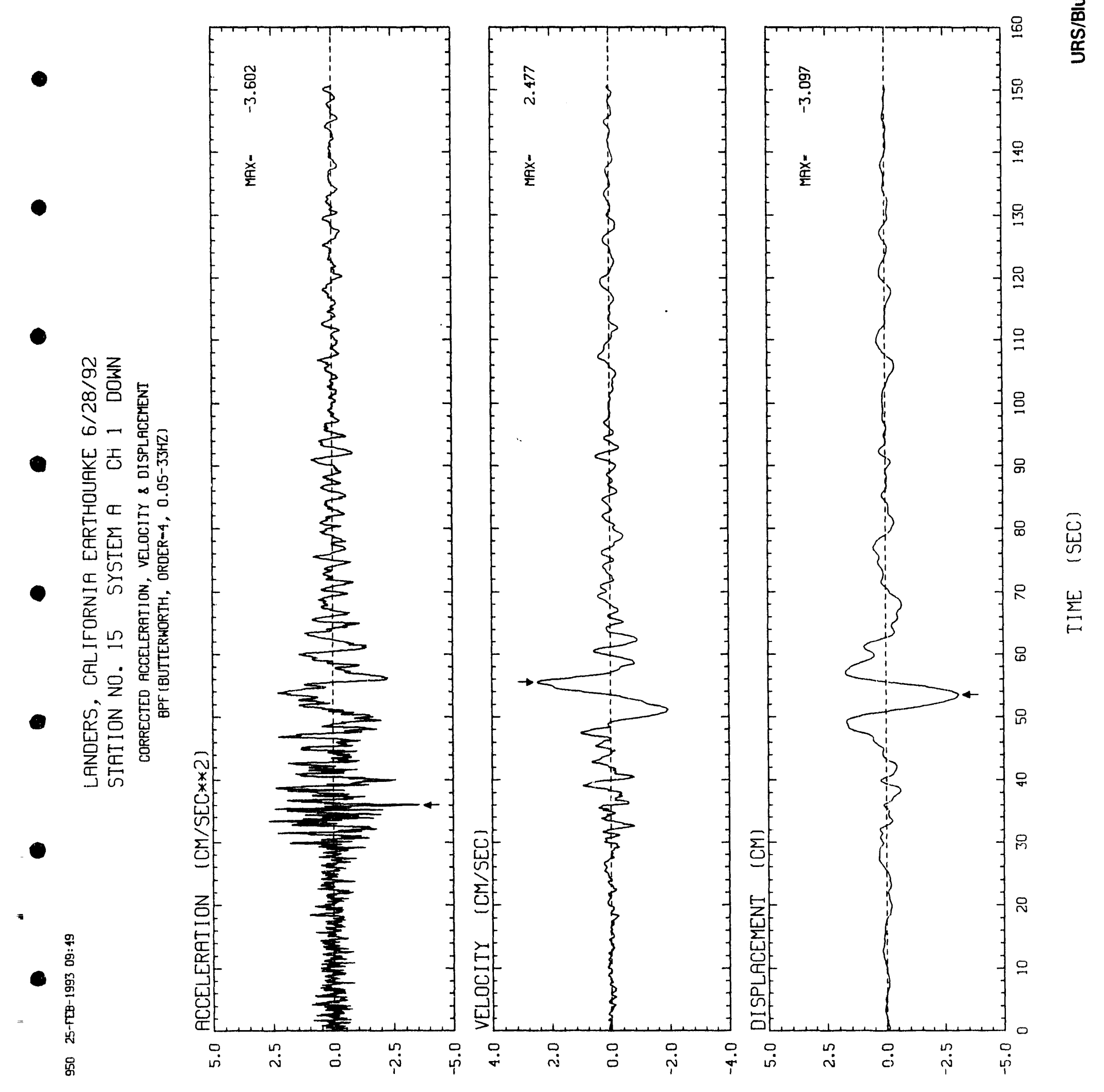

JOกII TdWH 


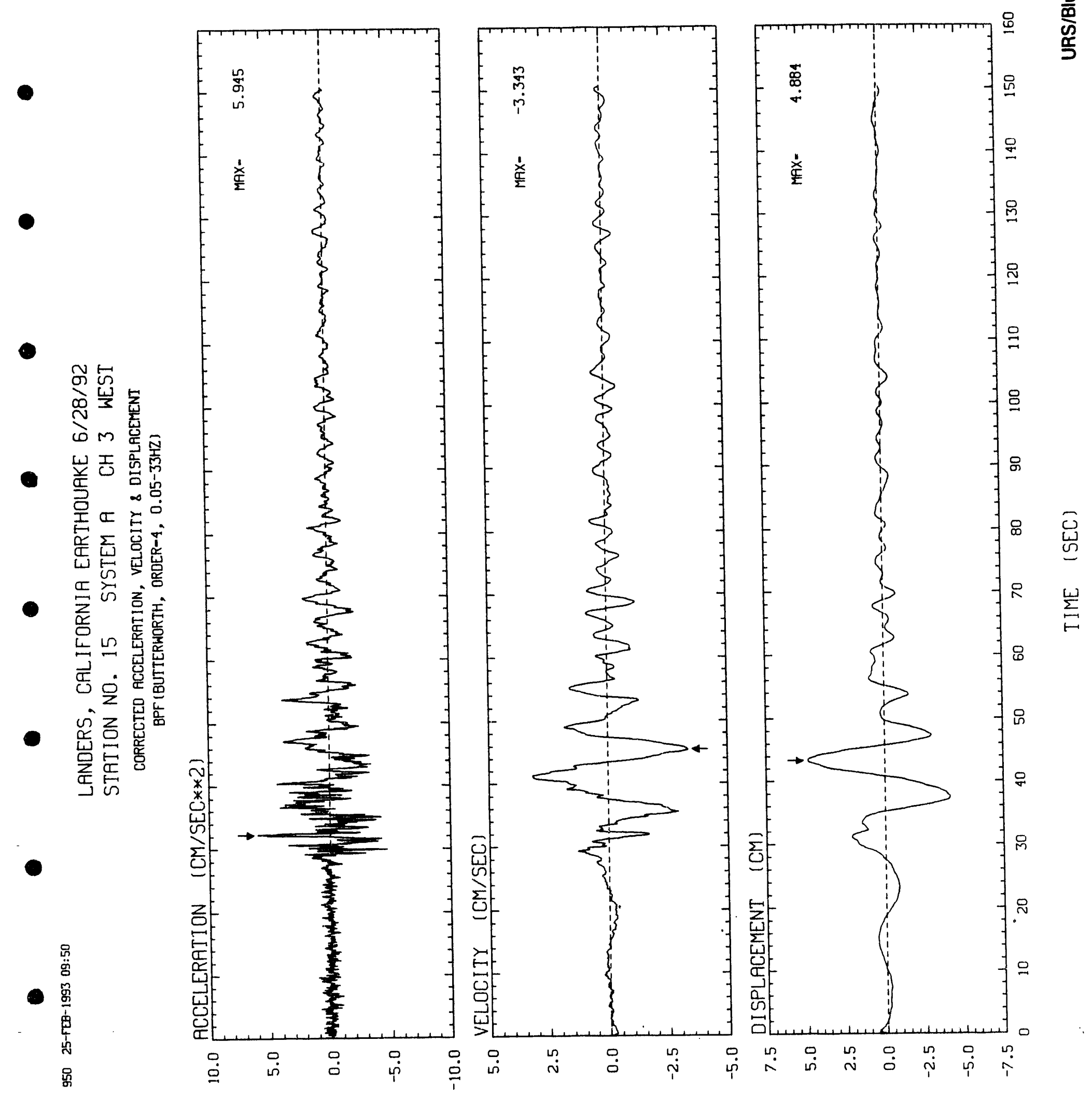

JดกI I 7dW 

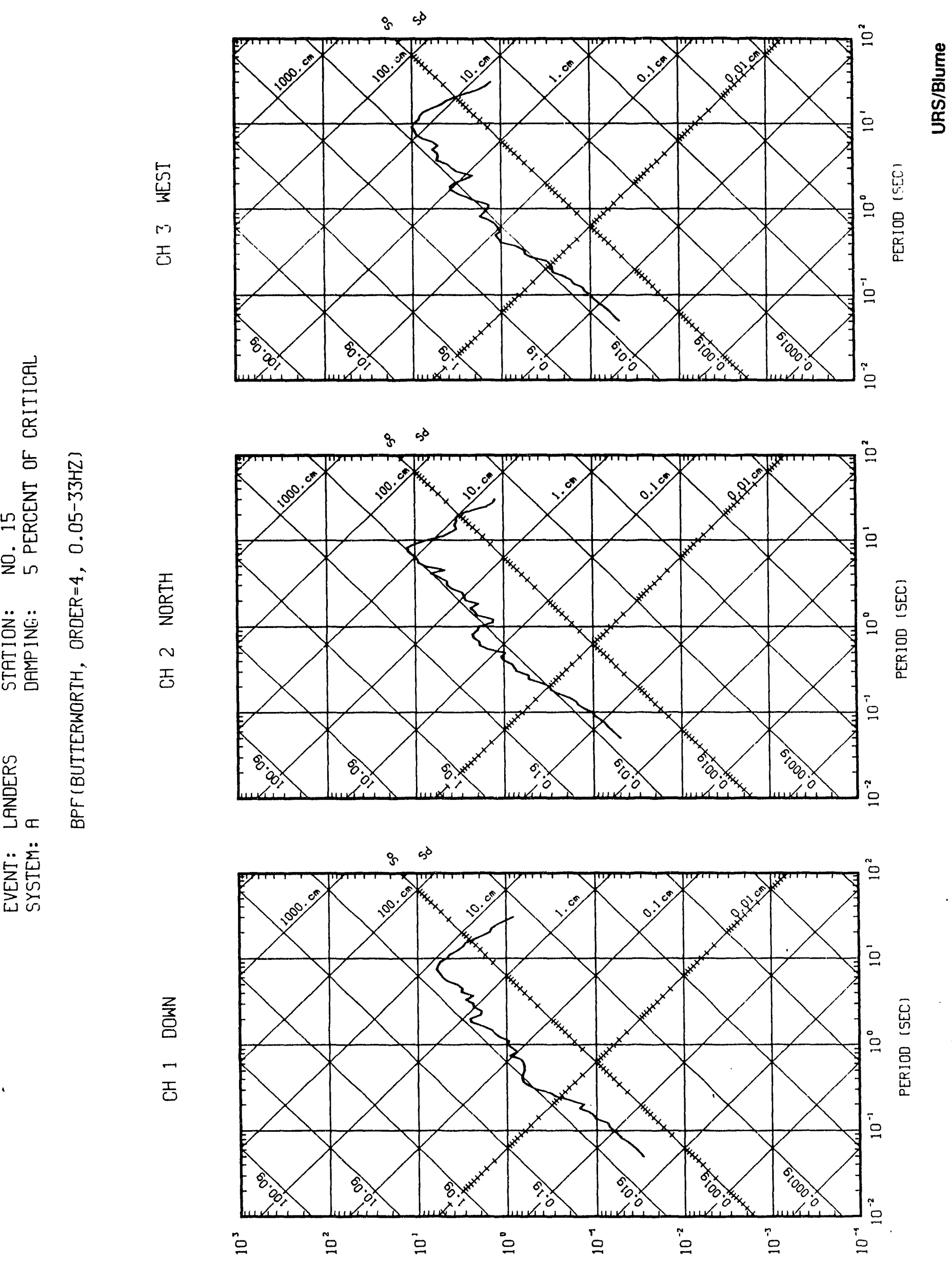

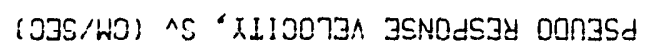




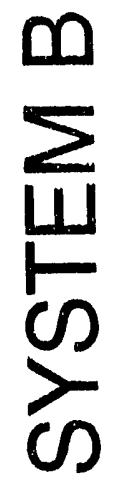

음

ำ

点

5 


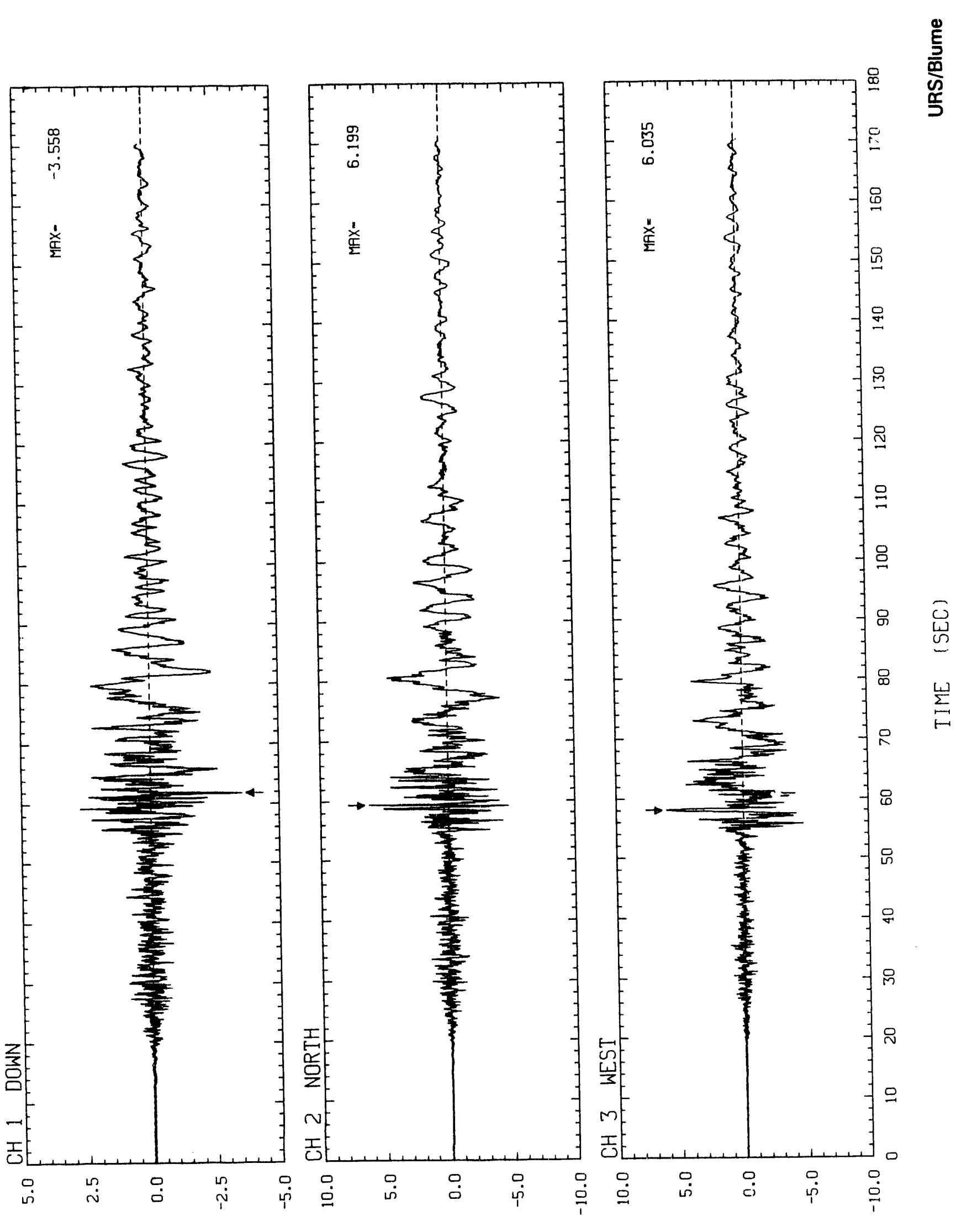

(2**03S/WO) NOIIHบ3า $303 \forall$ 


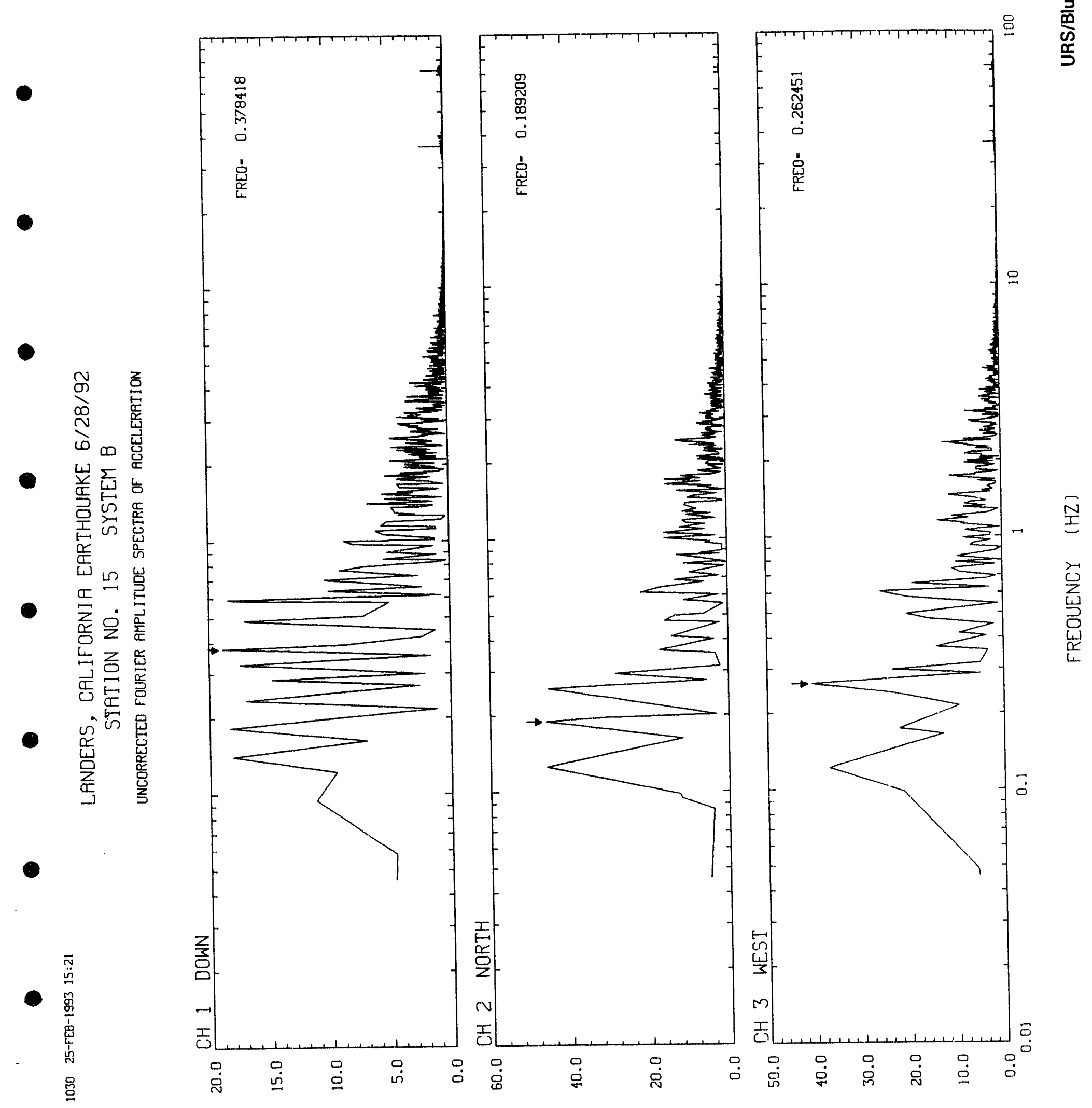

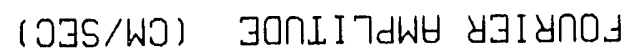



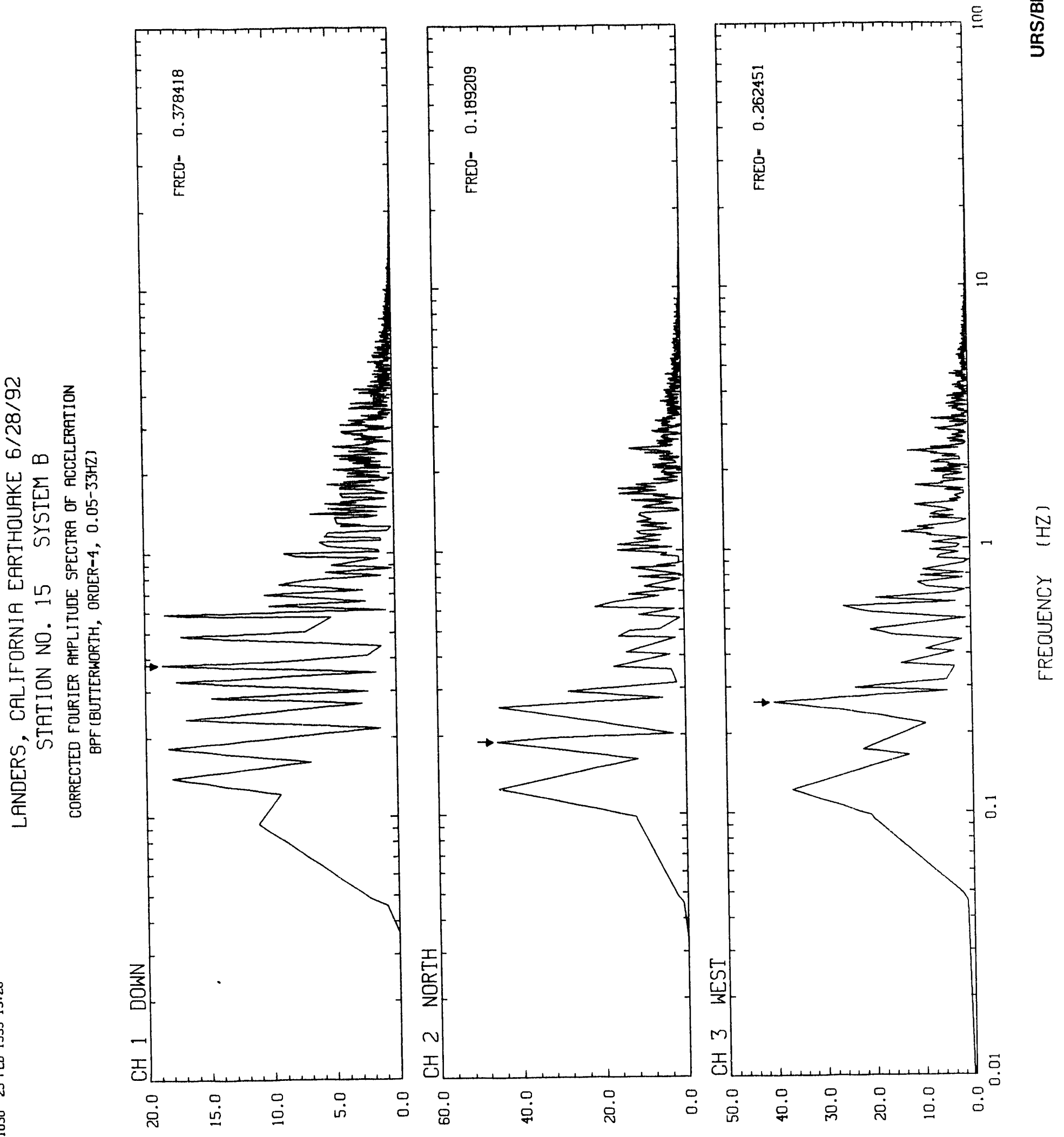

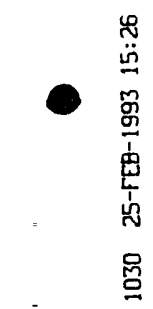

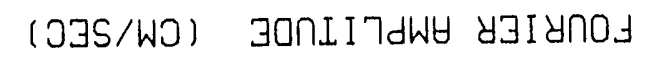




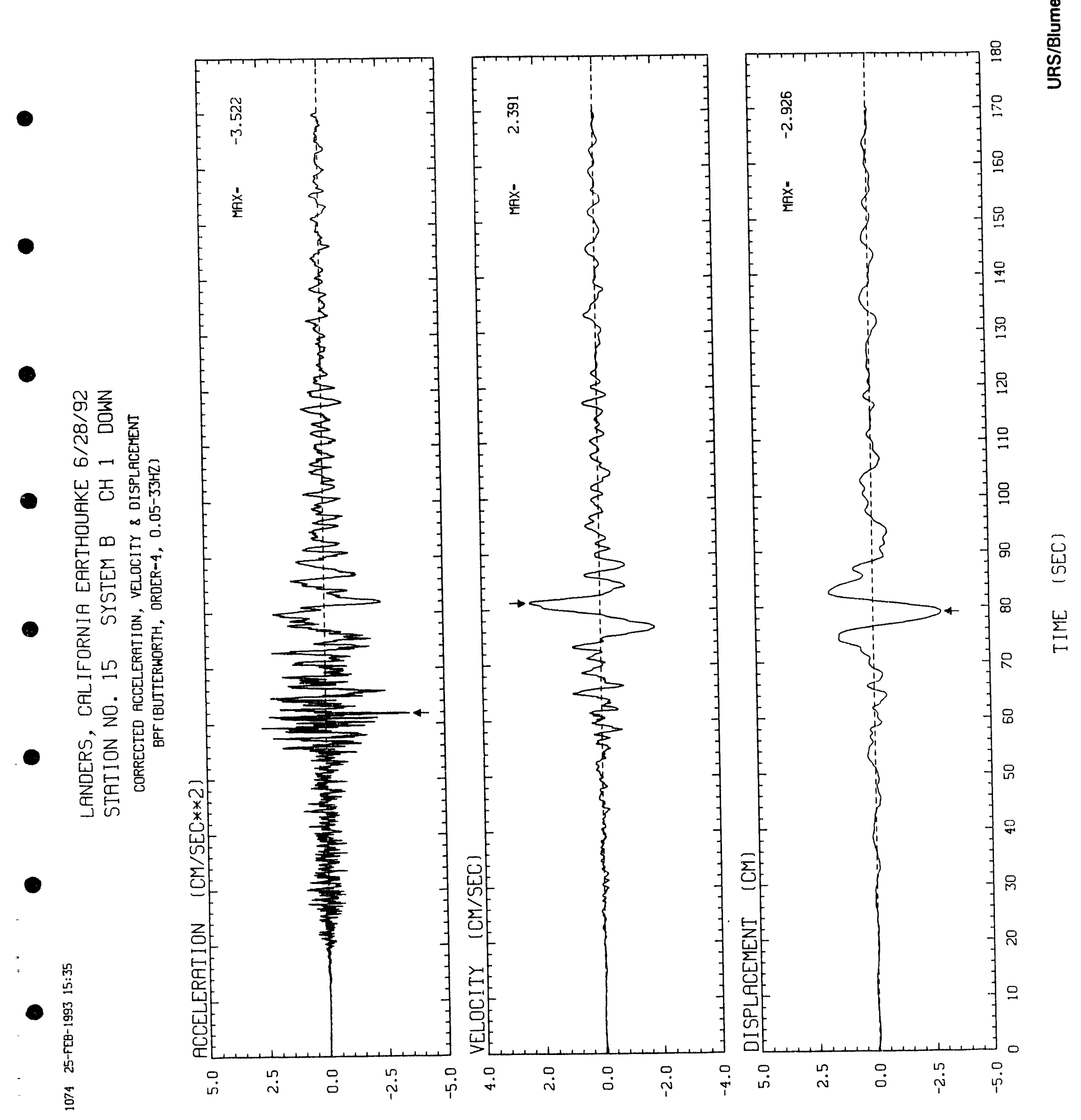



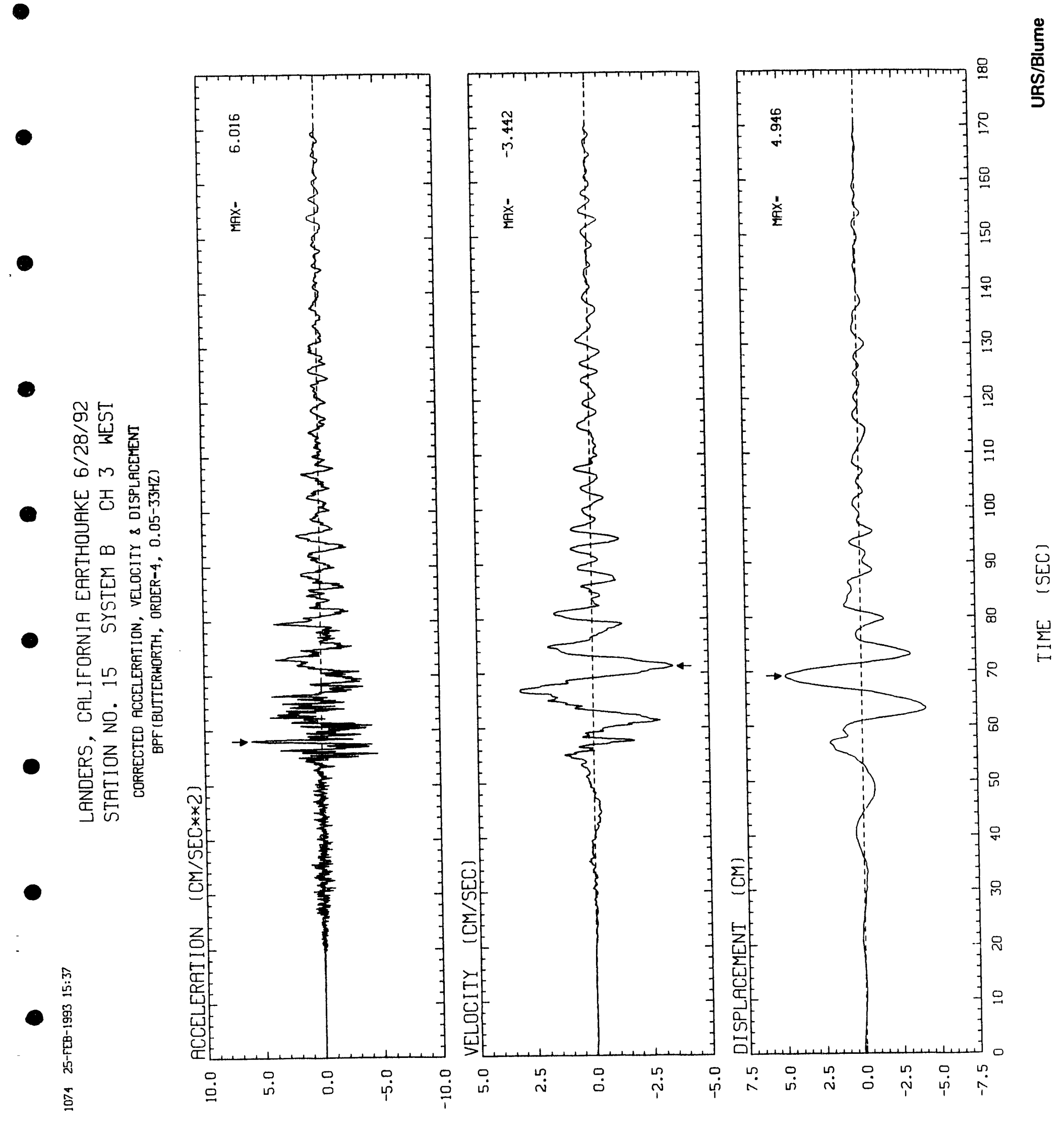

-

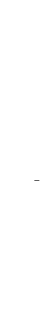



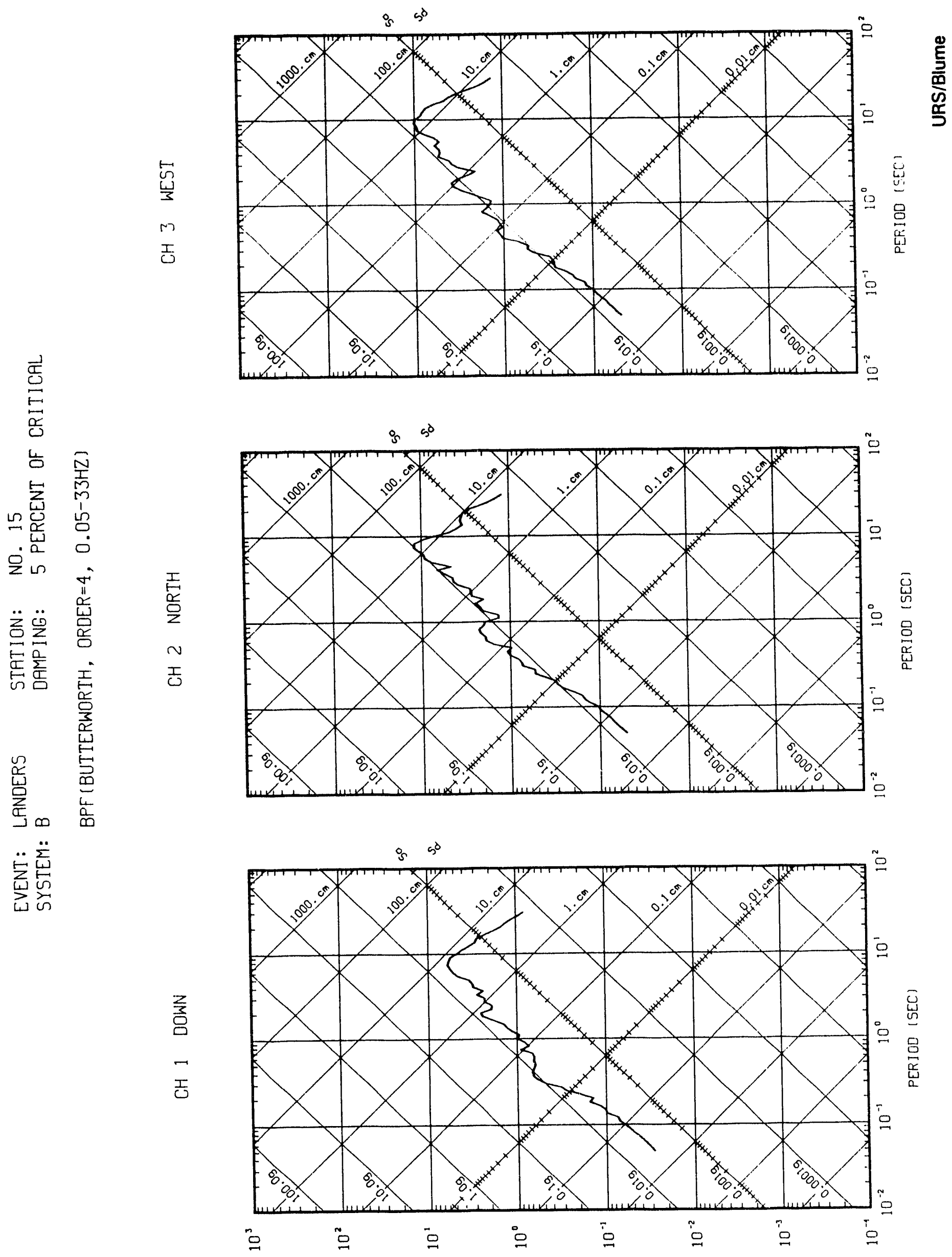

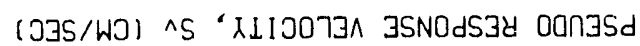


-

- 


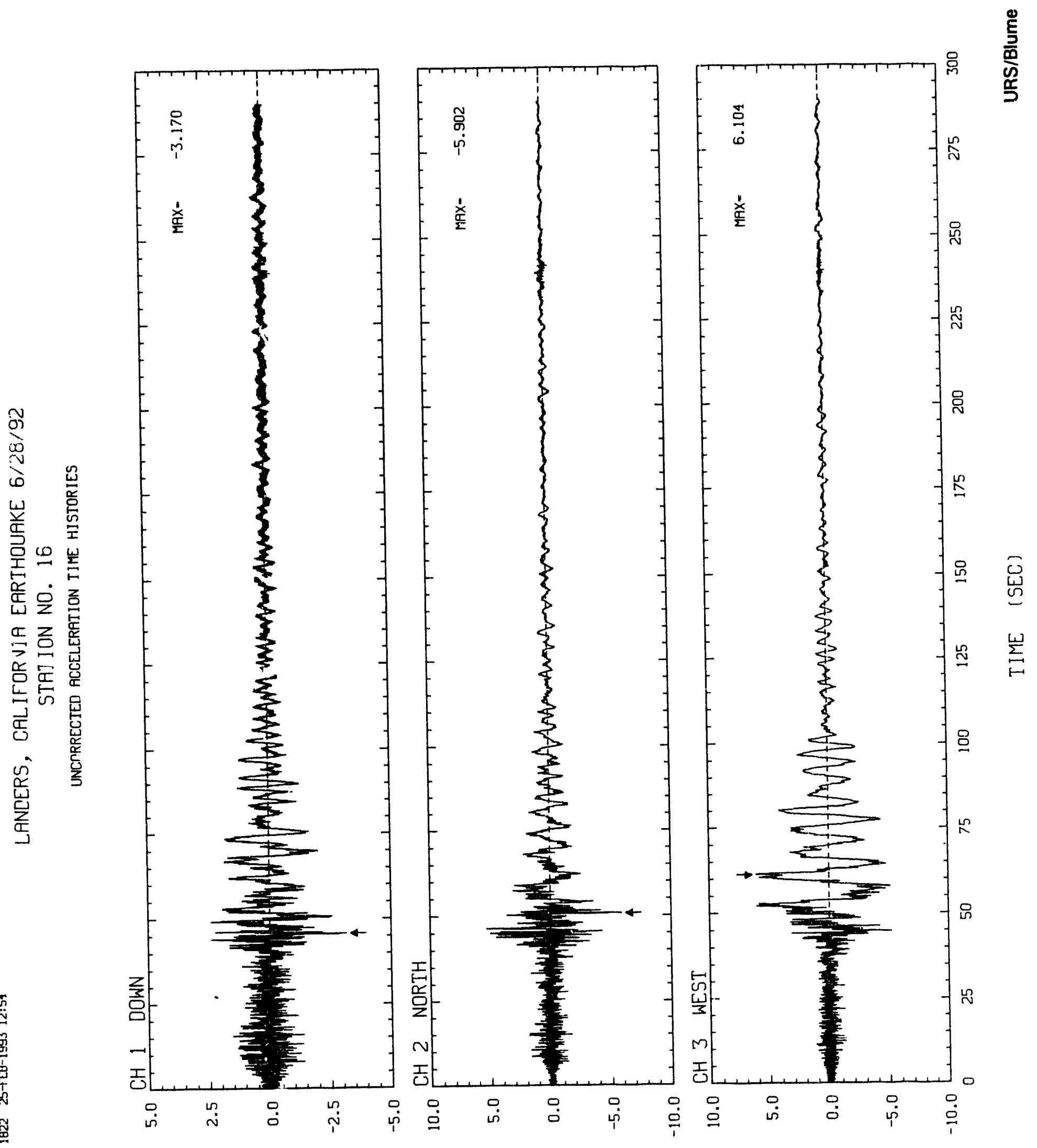

(2**375/WJ) NOII 


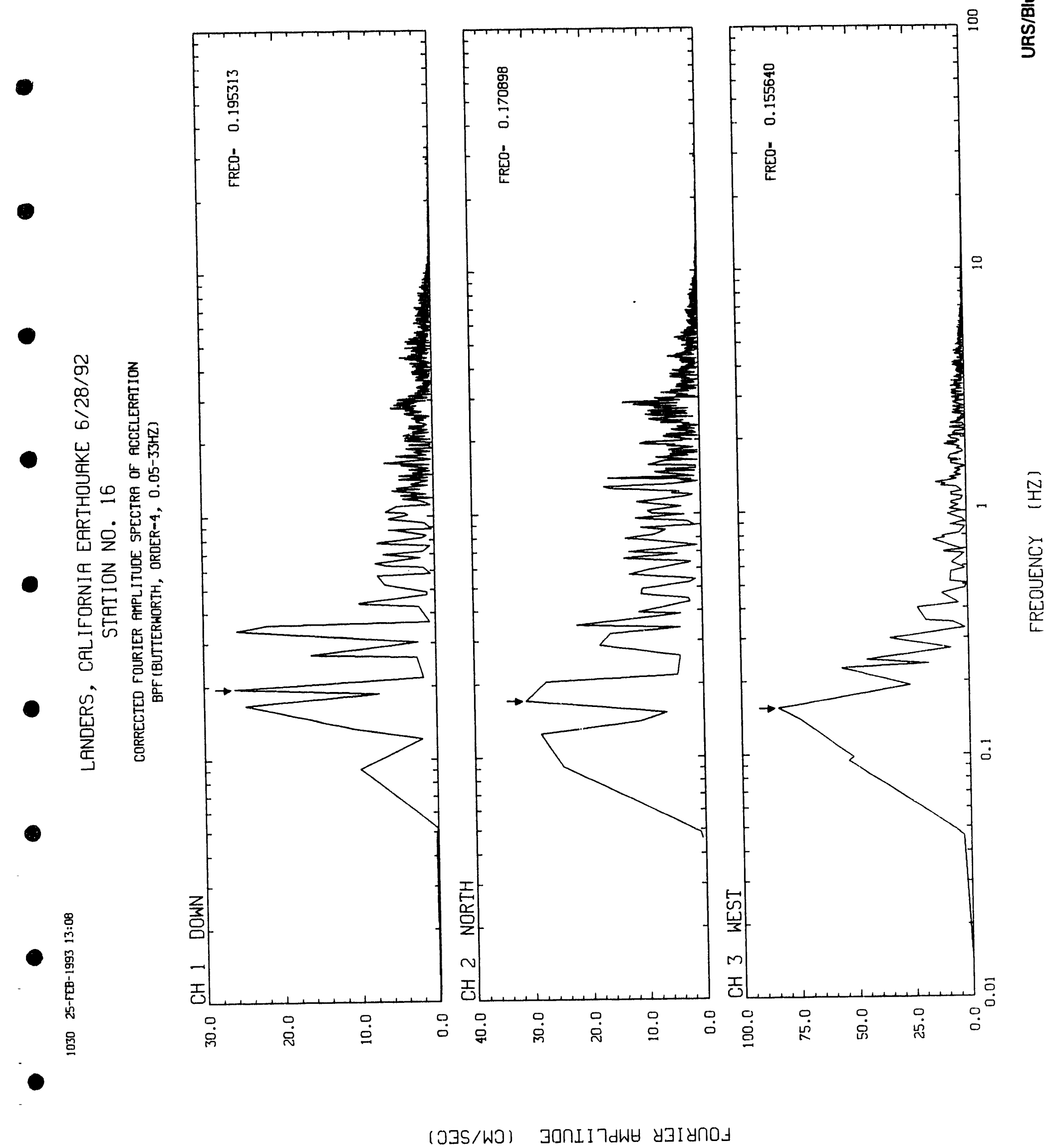




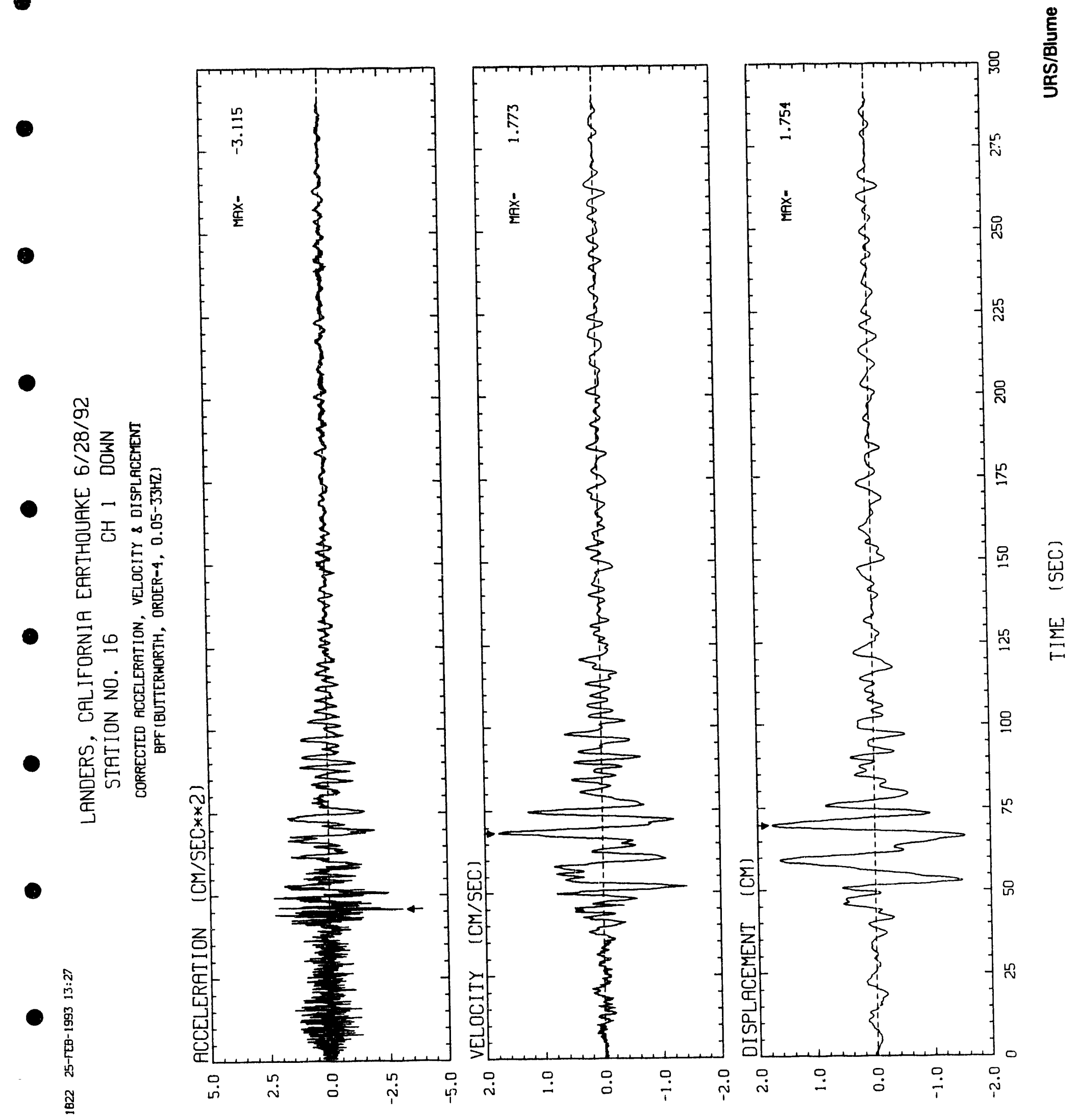




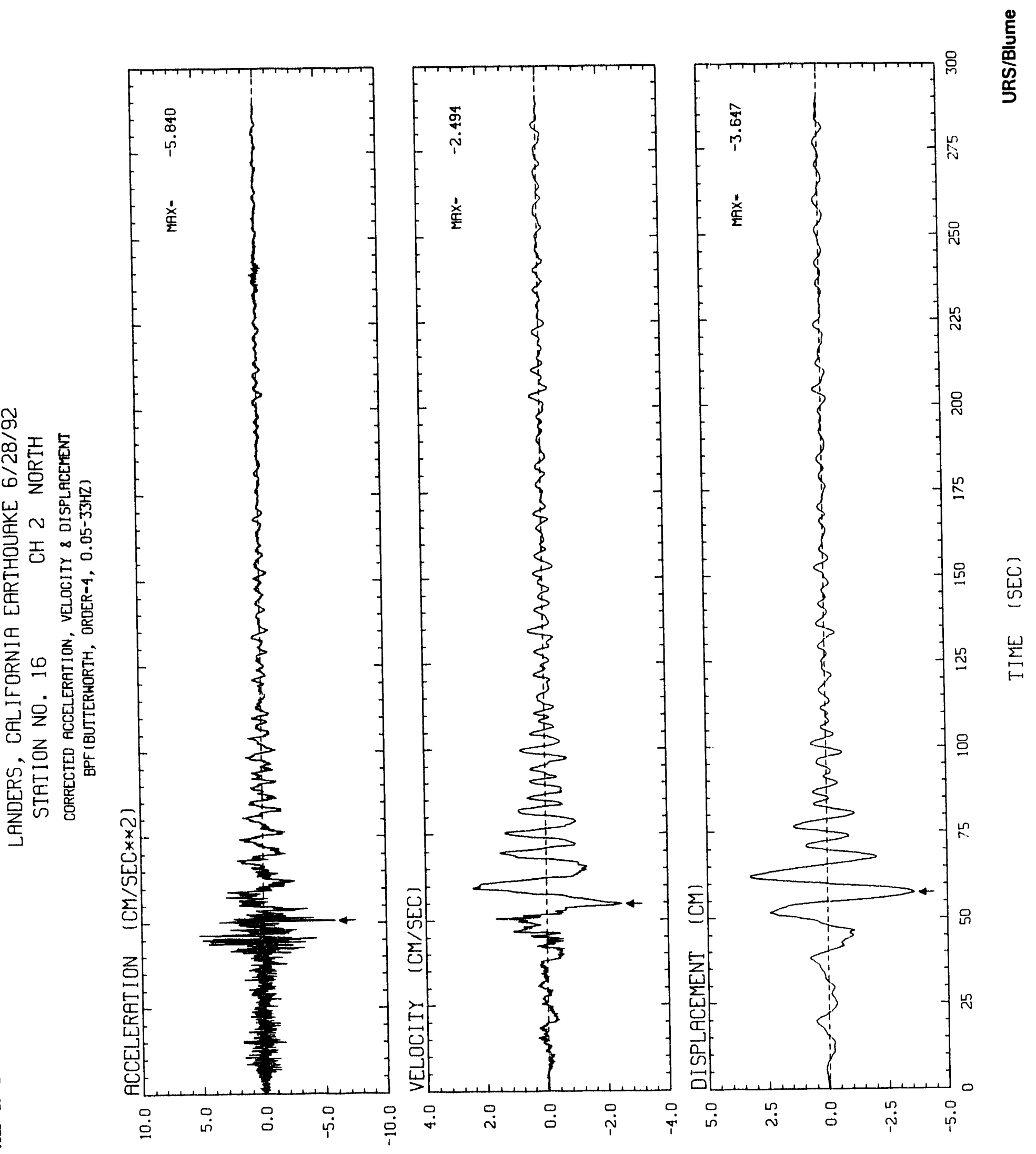

JOก.LI 7dWH 


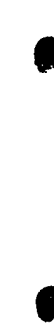




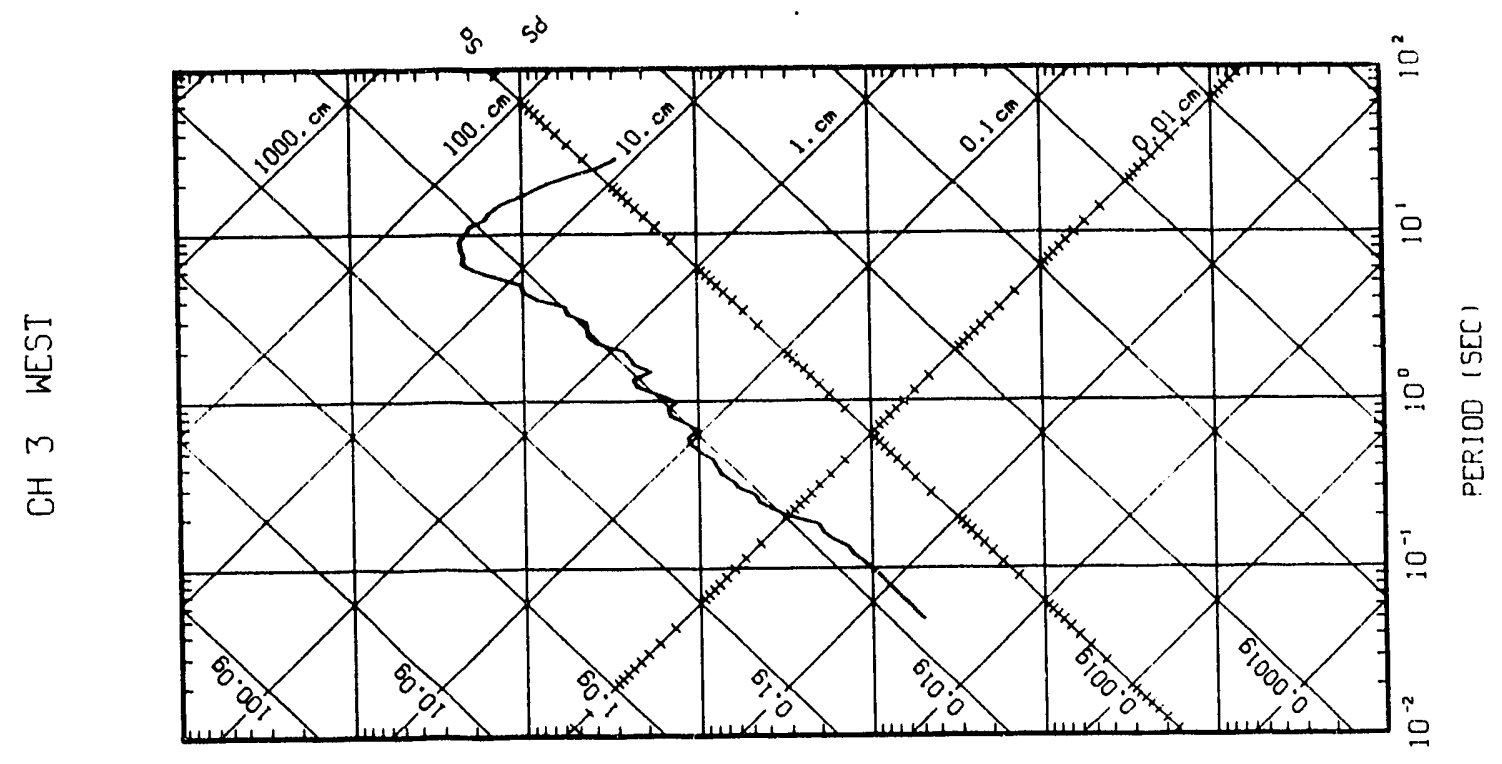

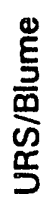
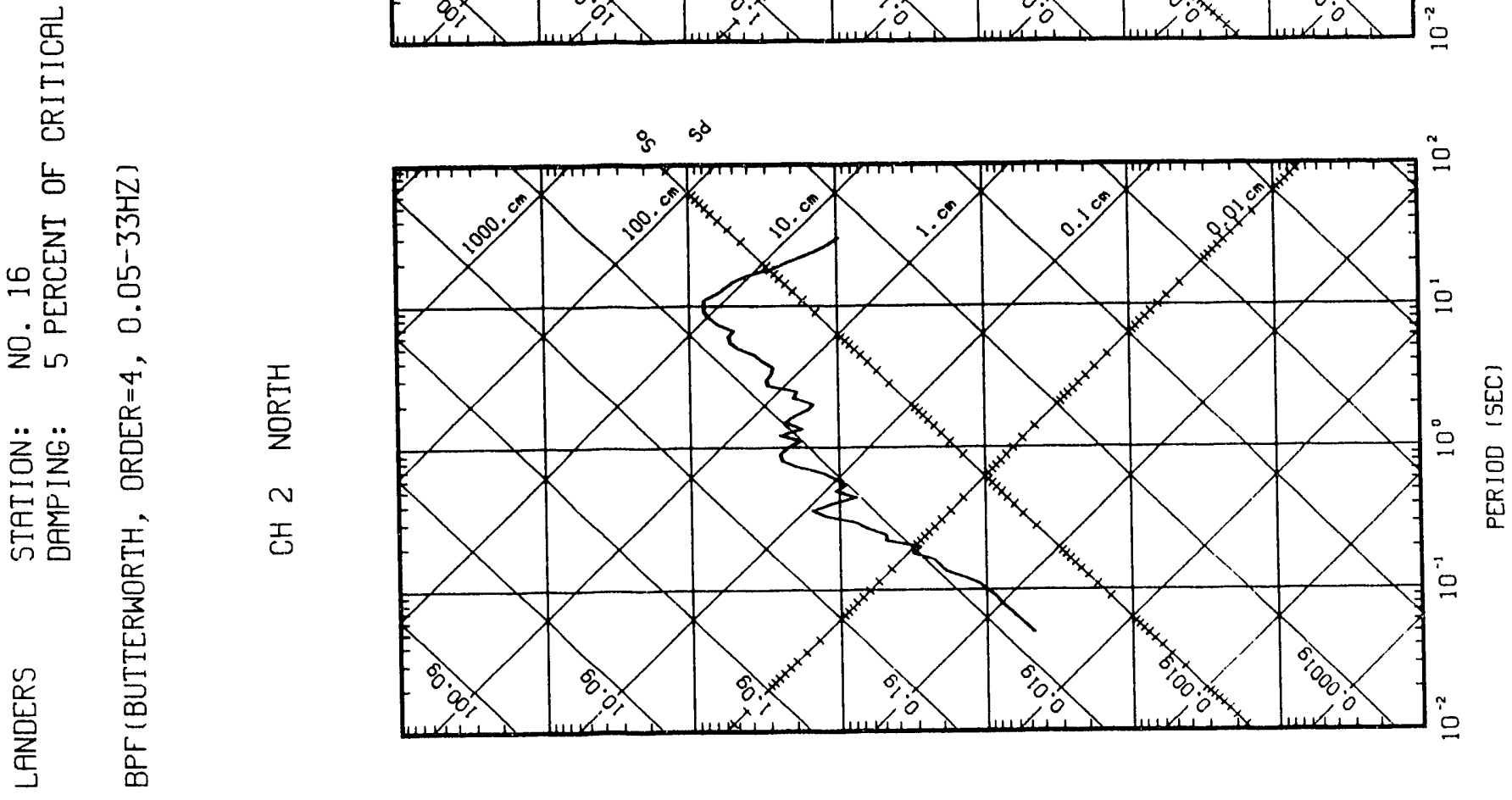

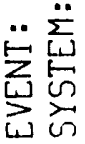

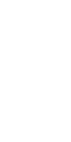

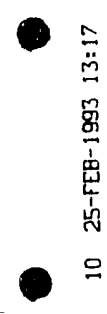

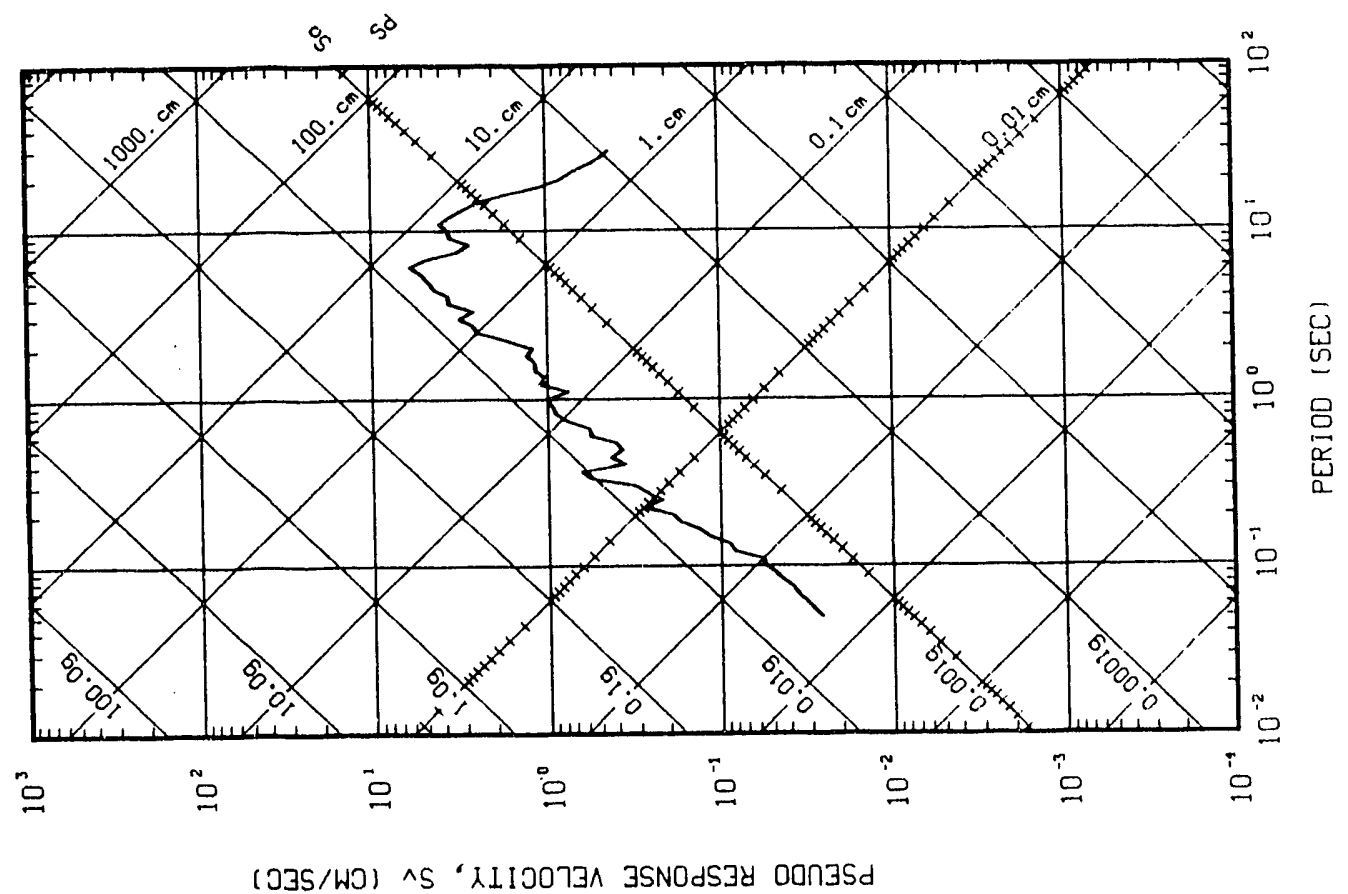


$\varangle$

$\sum$

ш

$\bar{D}$

c

든

운

Z

F

क

-

0

- 


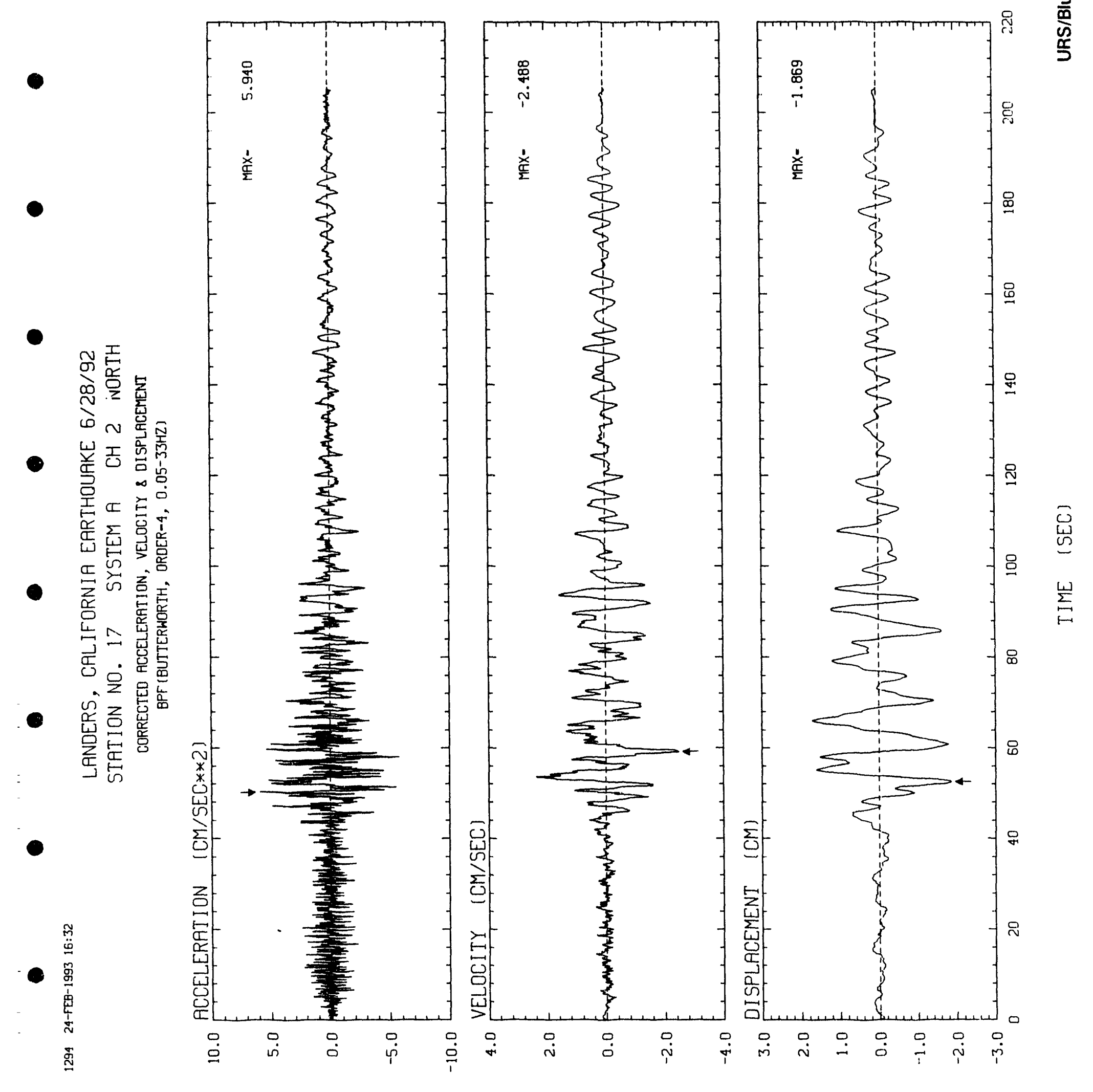

JOnI I 7dWH 
$\bullet$

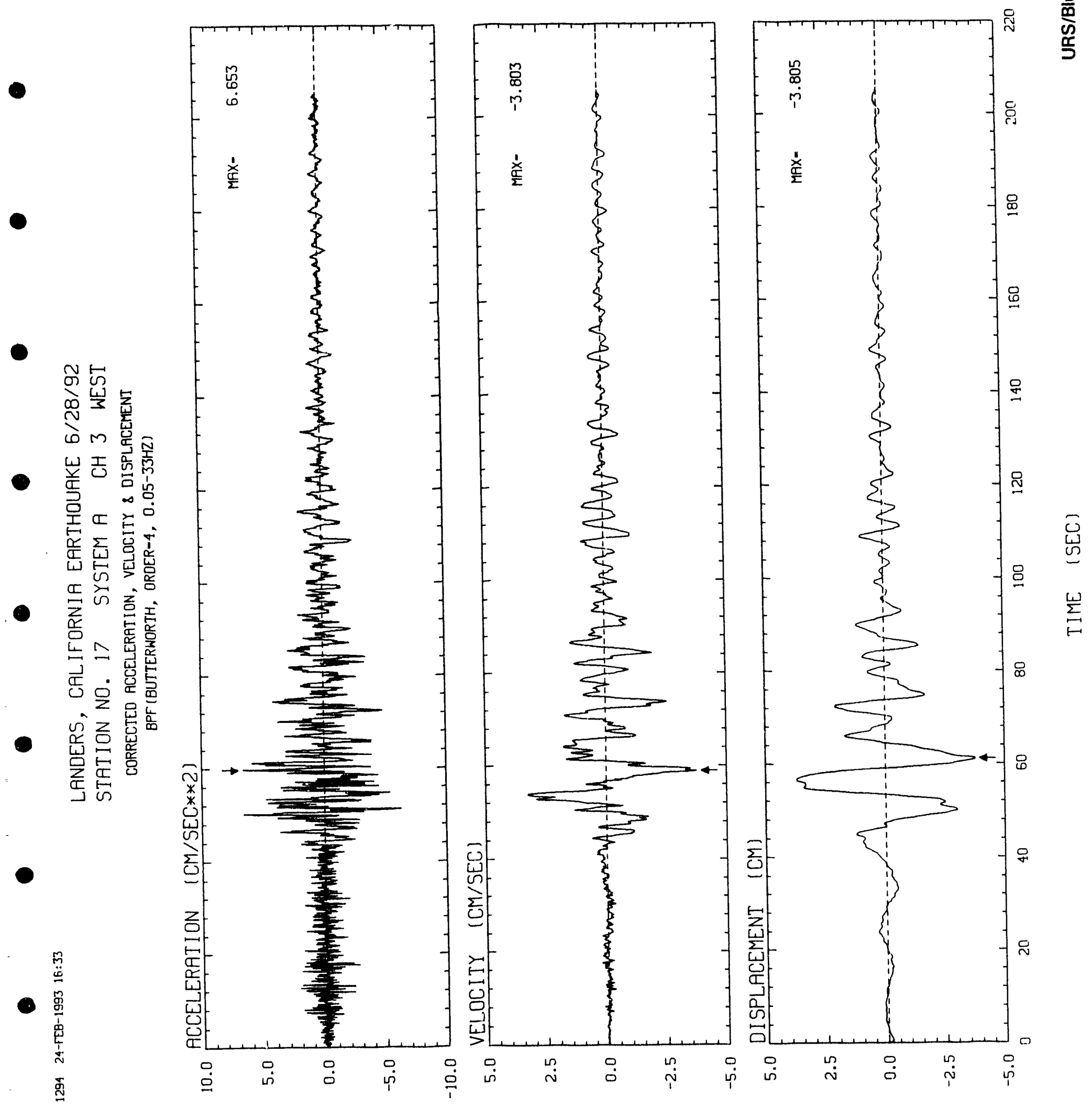

30กI I 7dW 


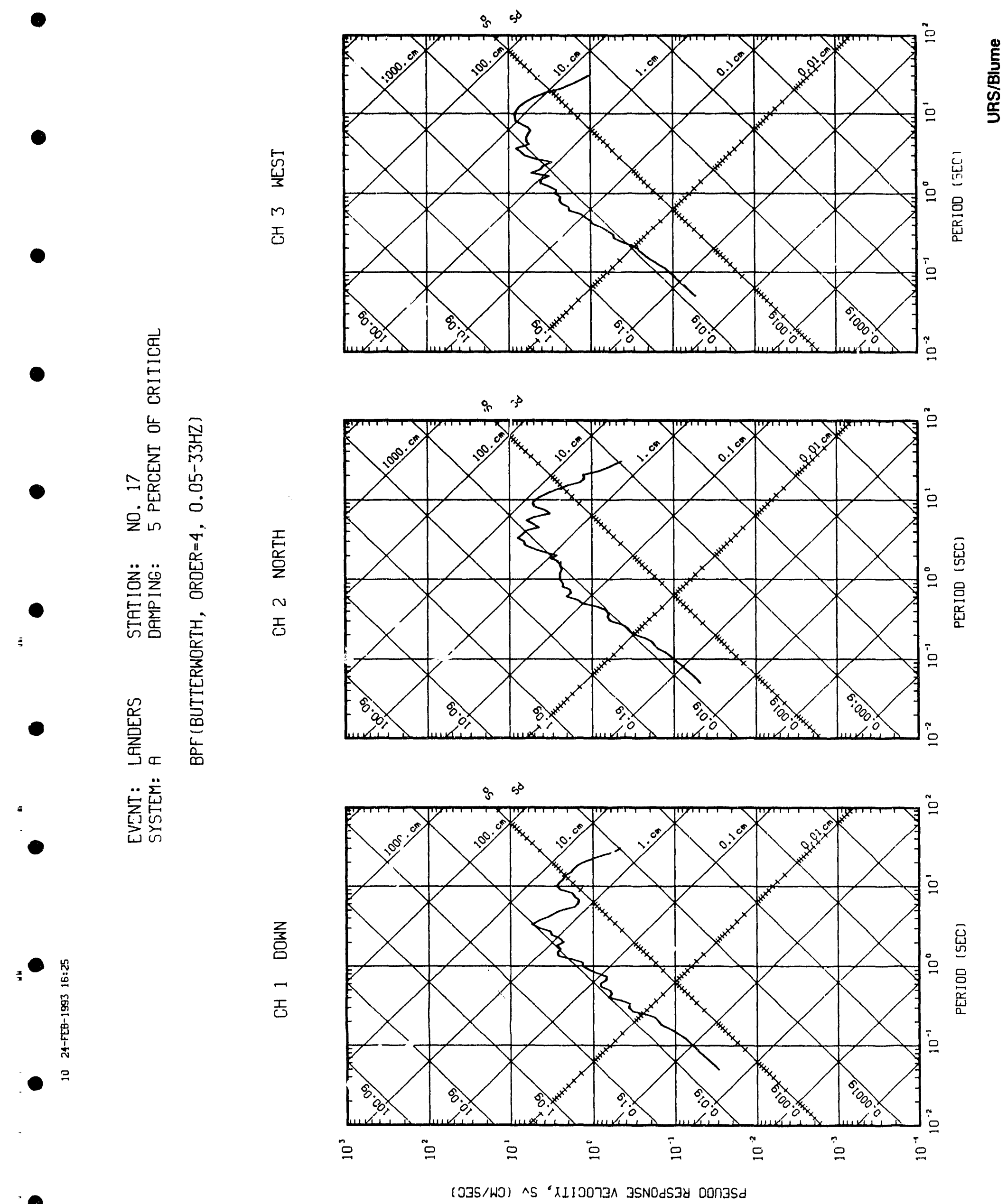


$\frac{\sum_{\infty}^{\infty}}{\infty}$

든

-

인

Z

O

心 
$\bullet$

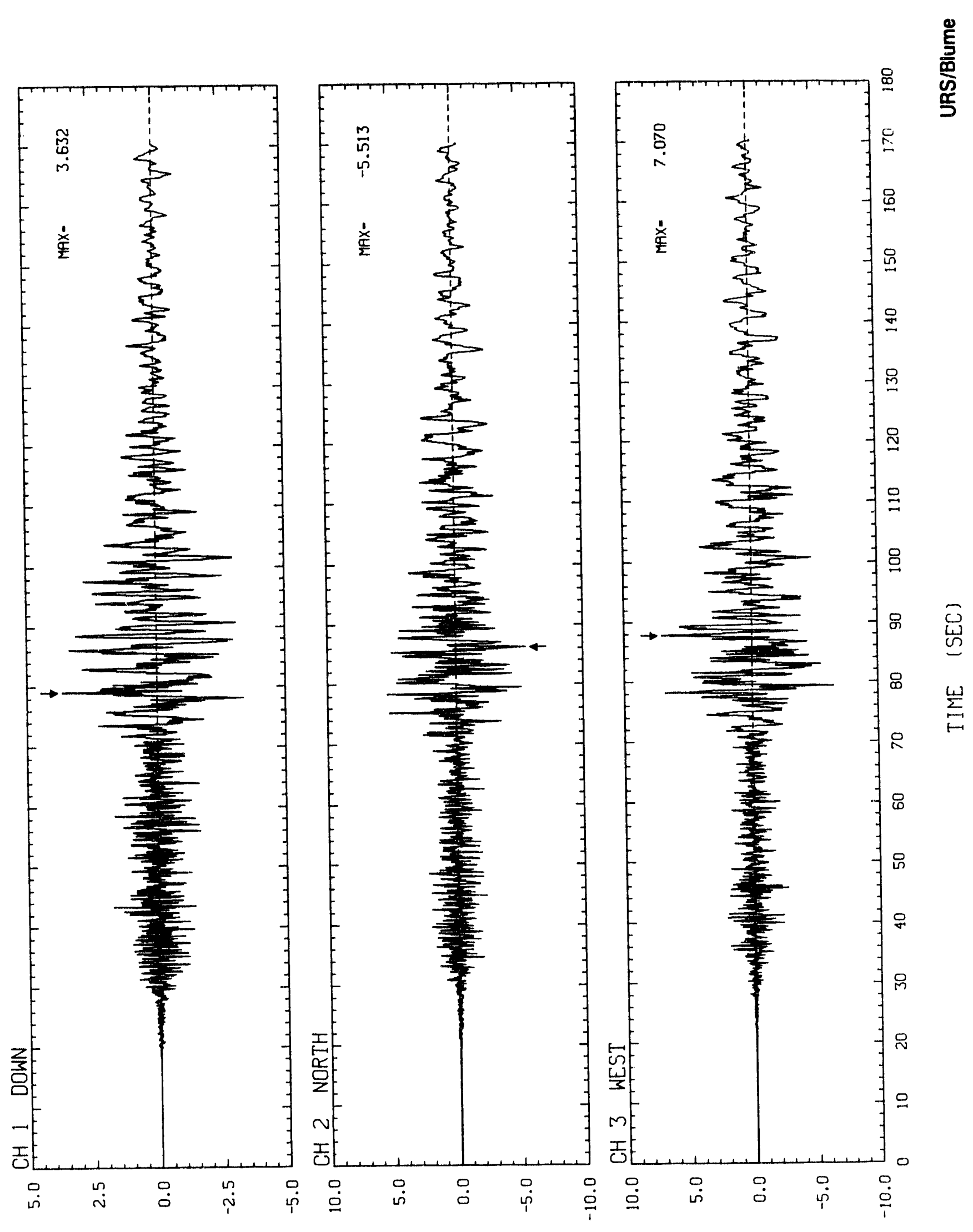

(2**035/WJ) NOI I $4437 \exists ว J \forall$ 


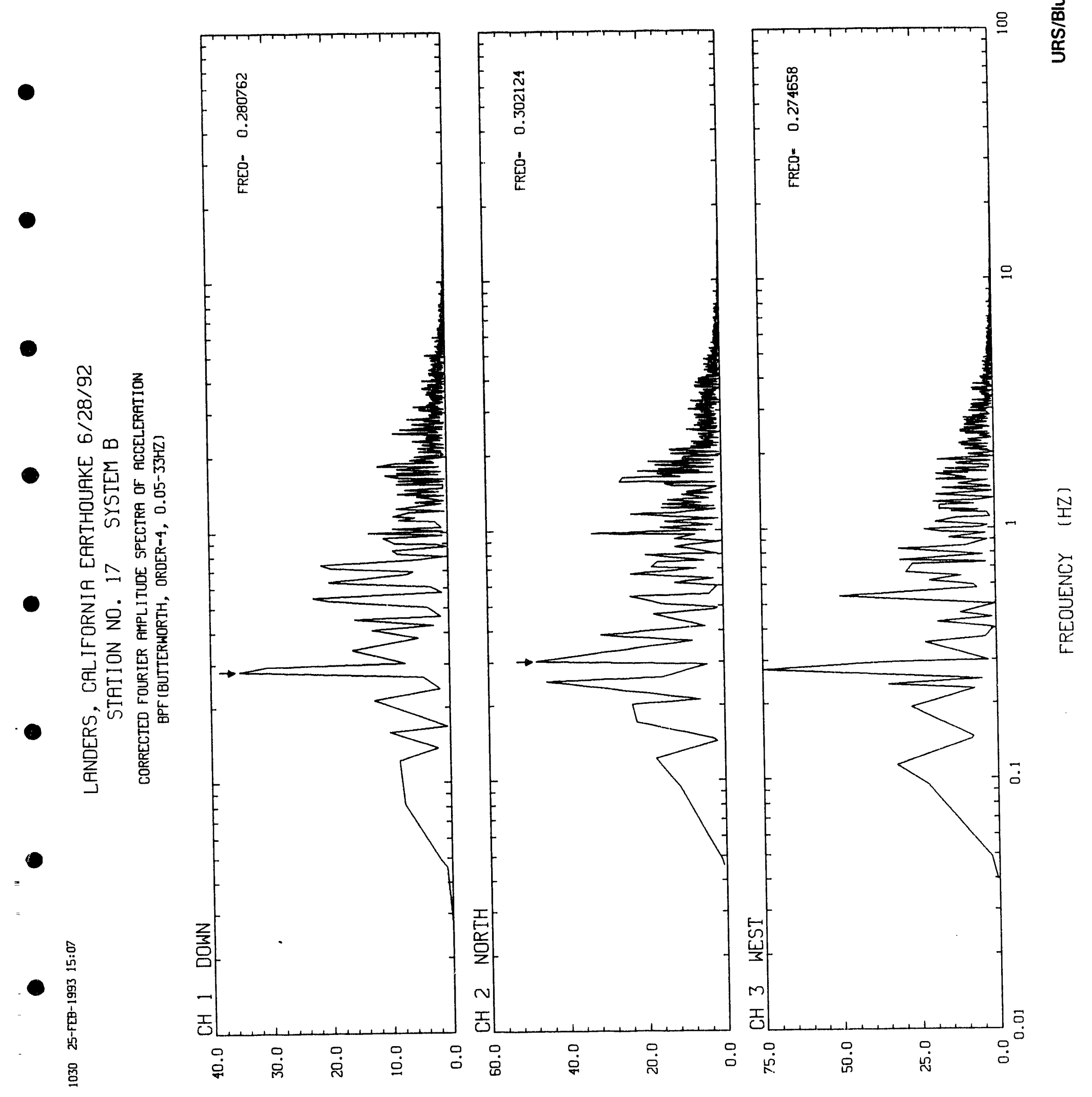


0

กั $\frac{I}{\text { ㄸ }}$

@

ம $\sim$ 峞

崖志芯喜

D $\infty$ 웅

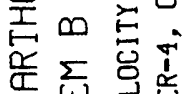

山岕岕岕

든

学宫点

느 岕亭

元

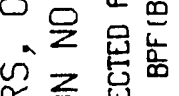

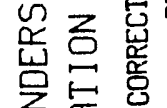

号志琮

O
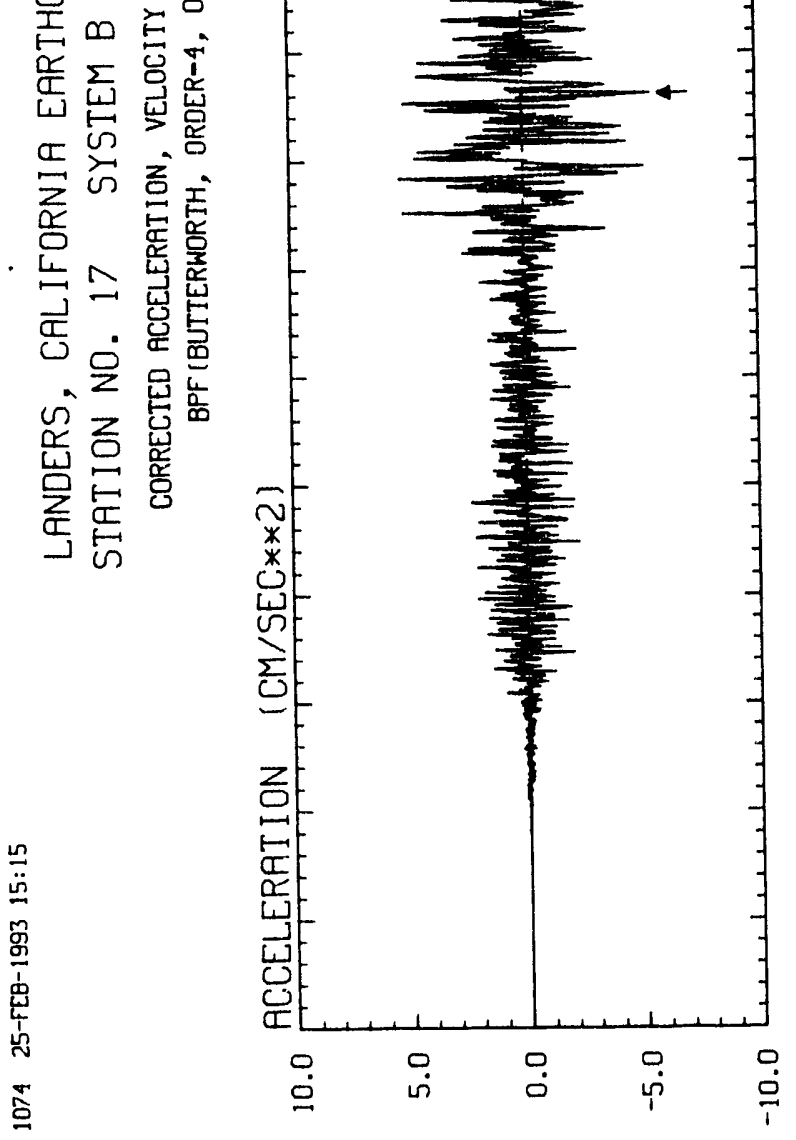

FE

랑.
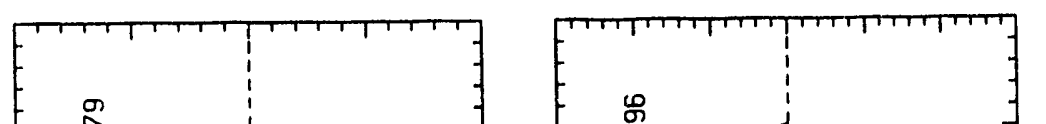

भे

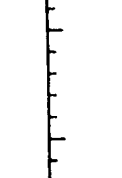

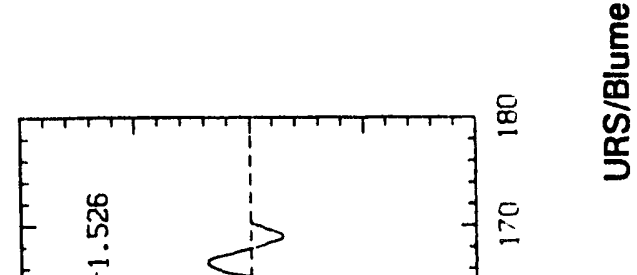

$\frac{\stackrel{D}{5}}{\stackrel{\oplus}{\frac{0}{5}}}$

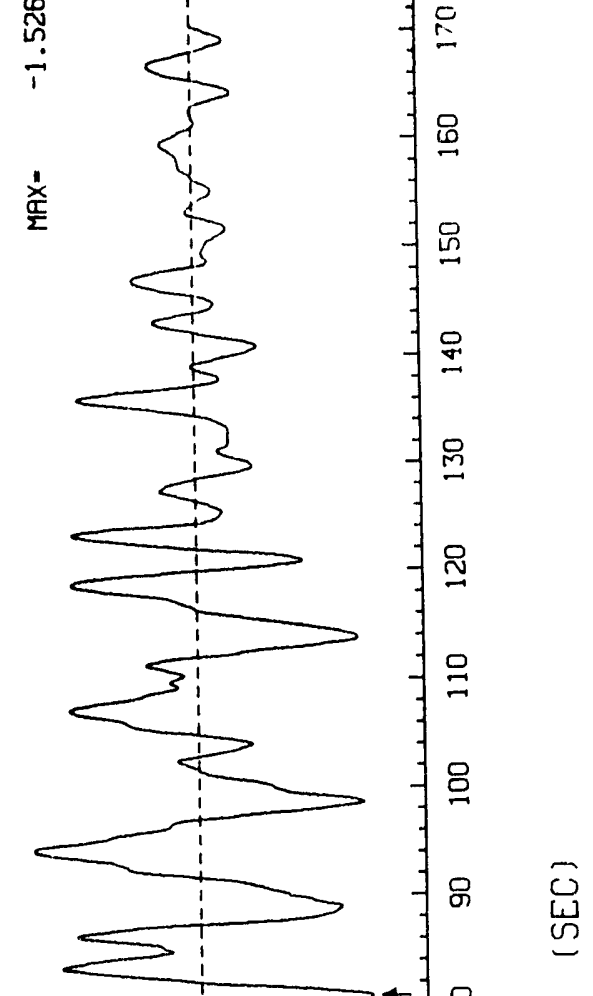

$\sum_{\models}^{W}$ 

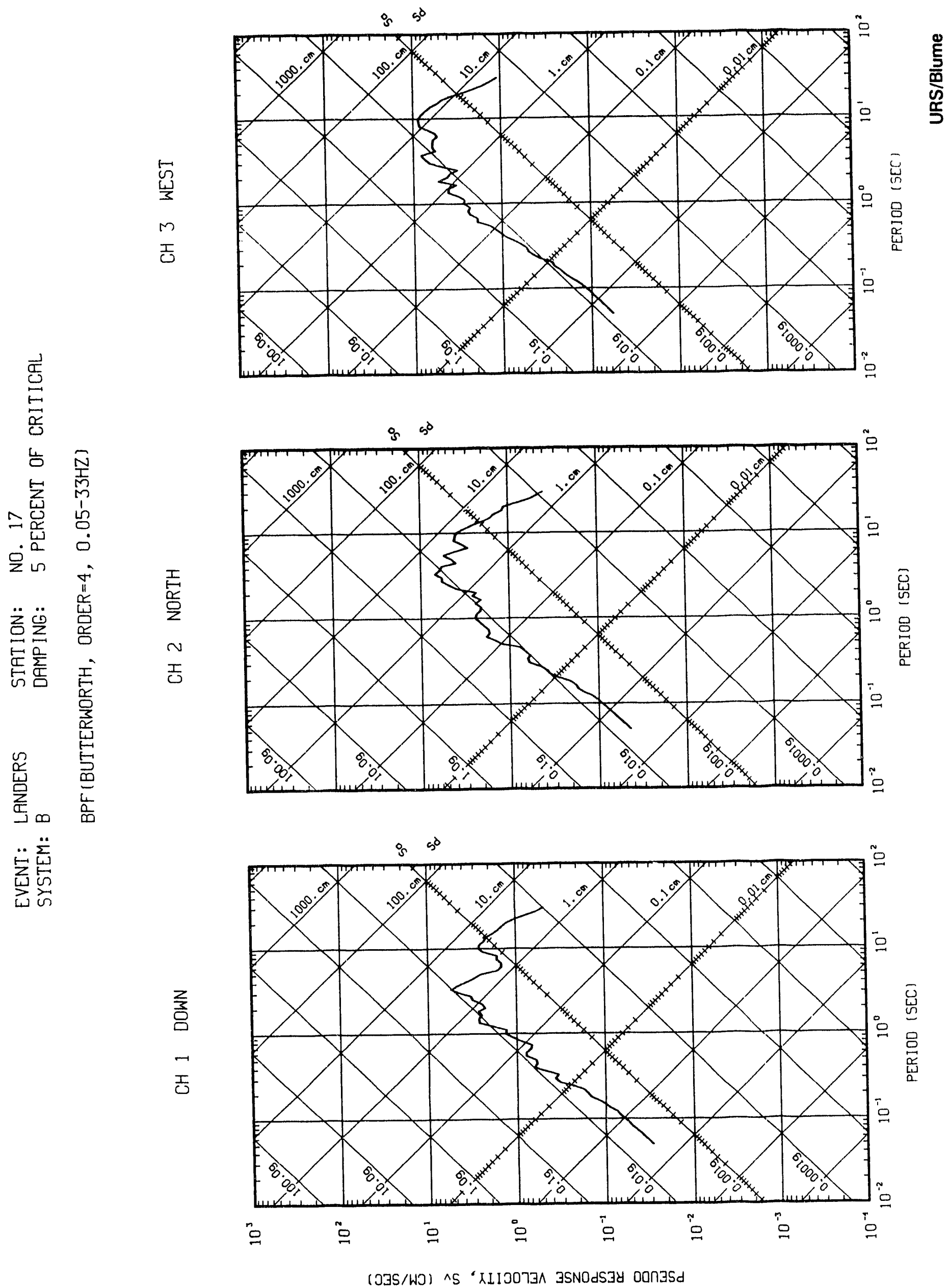
-

-

-

$\infty$

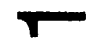
0

Z 
$\bullet$

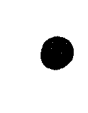

จิ

-

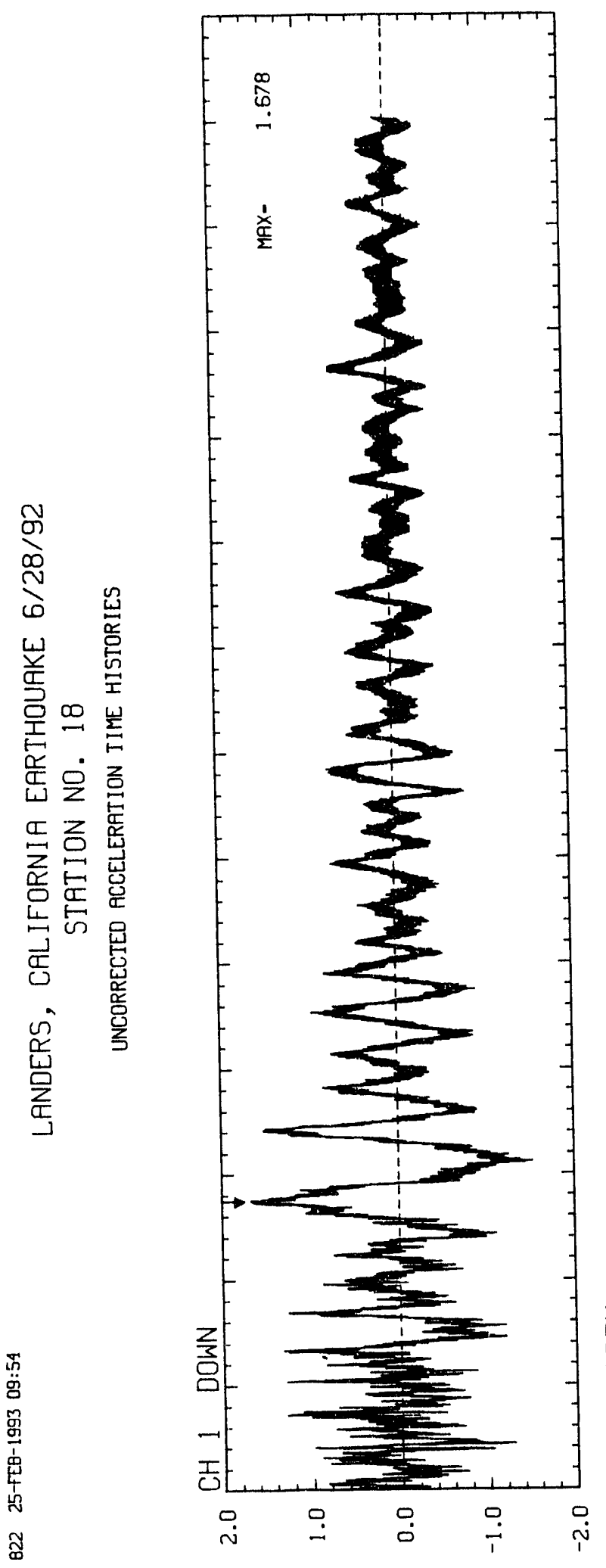

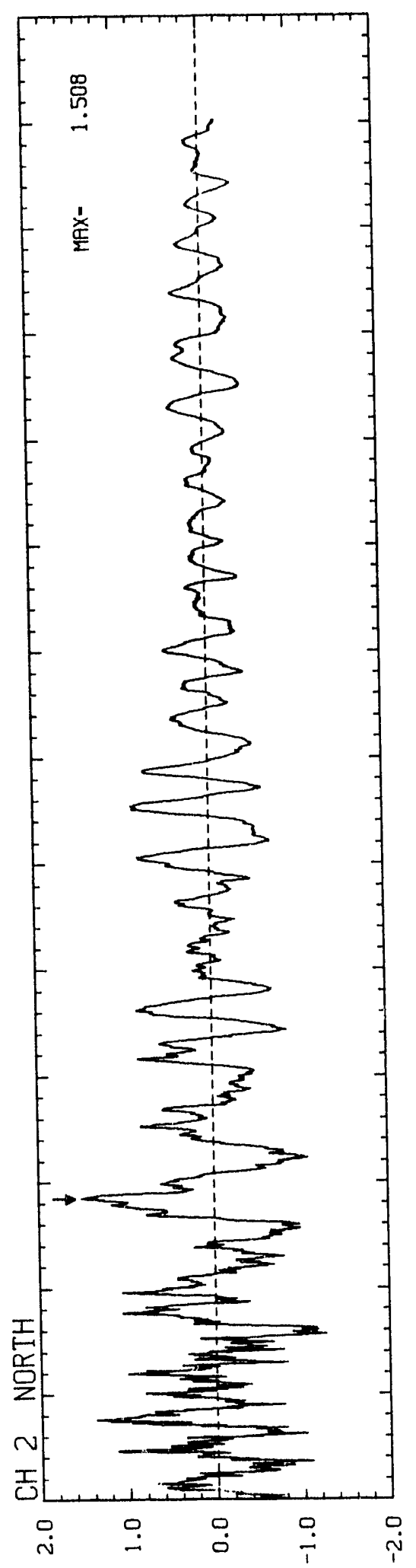

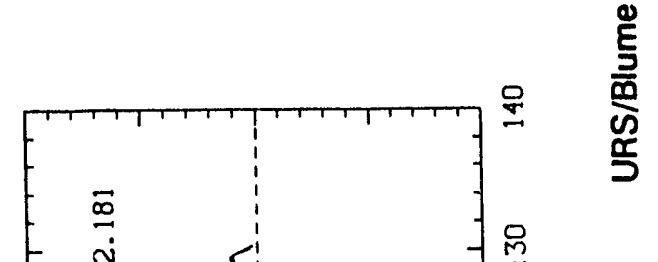

0
岂
$\sum_{E}^{U}$

오

암

오

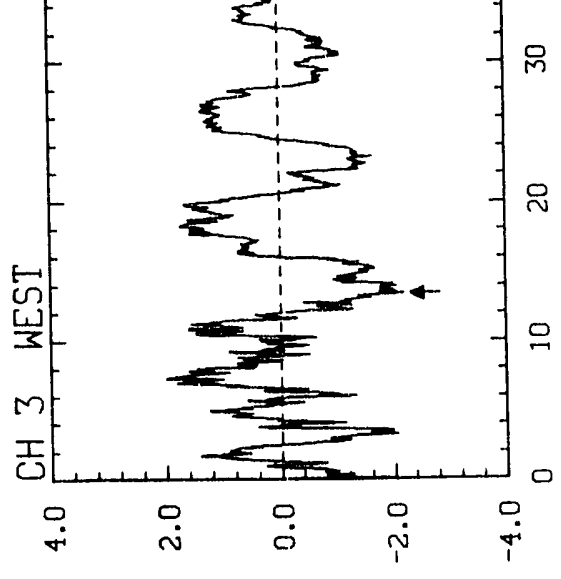

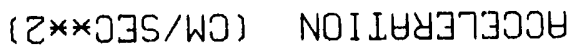




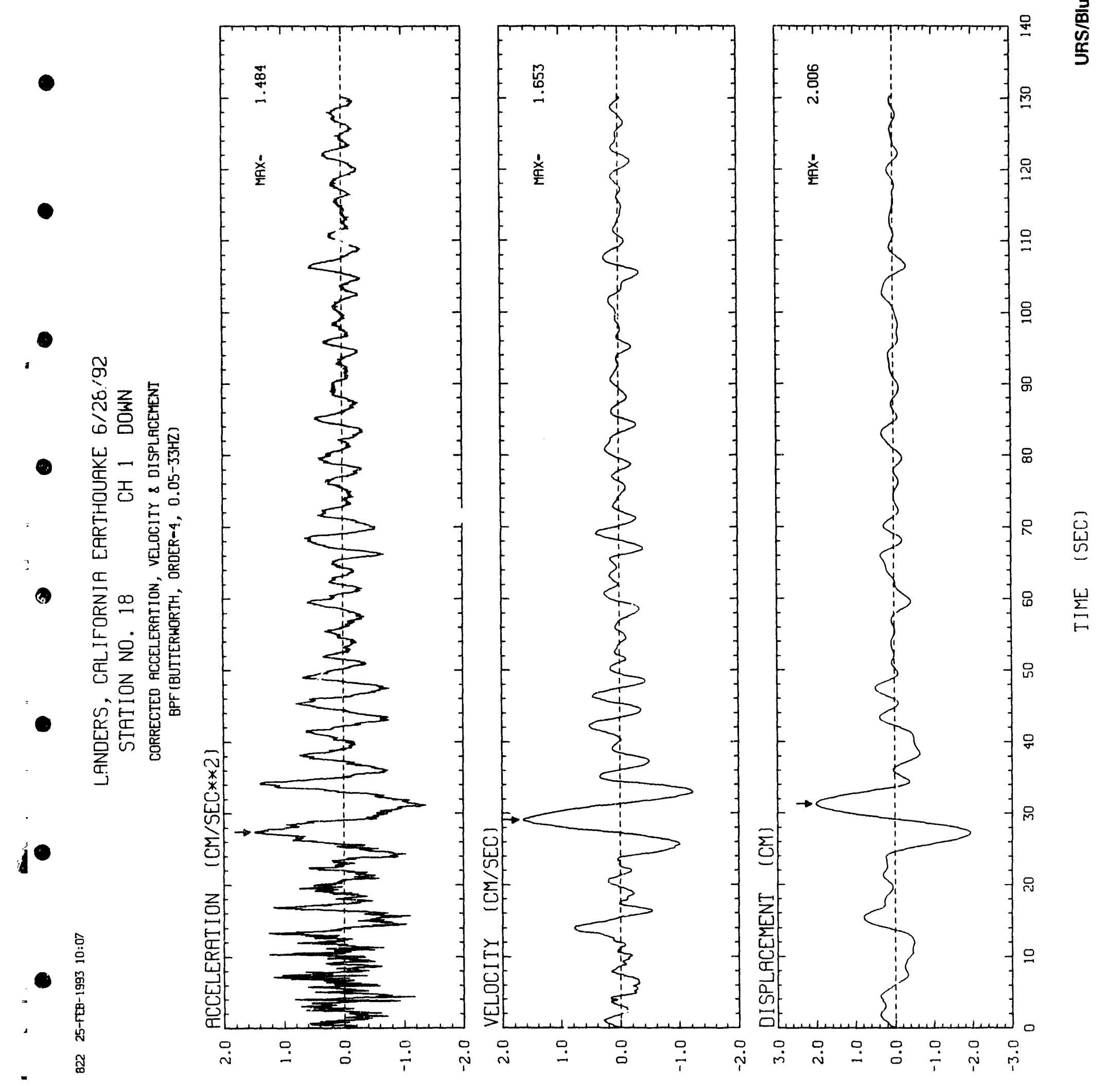




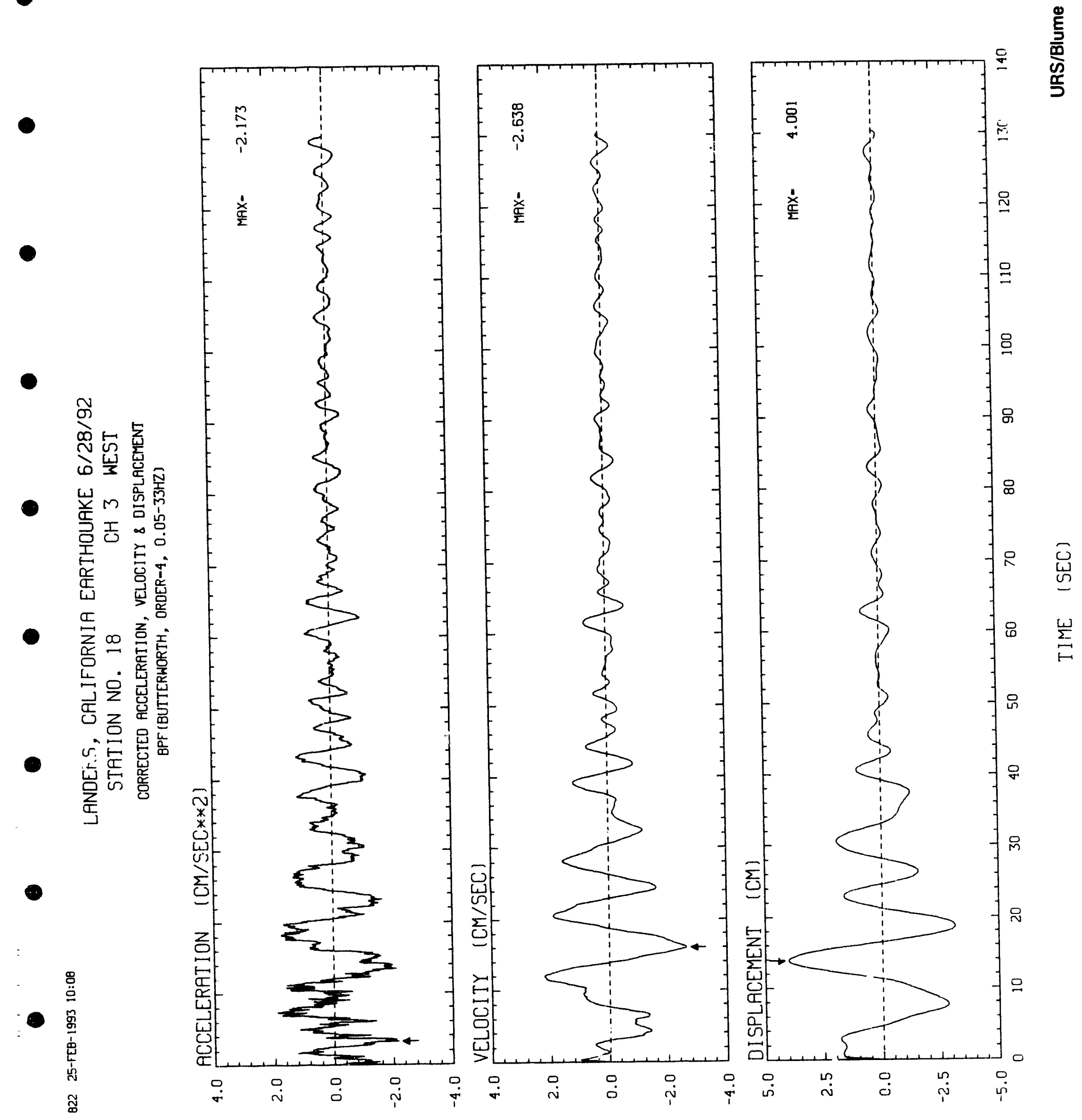




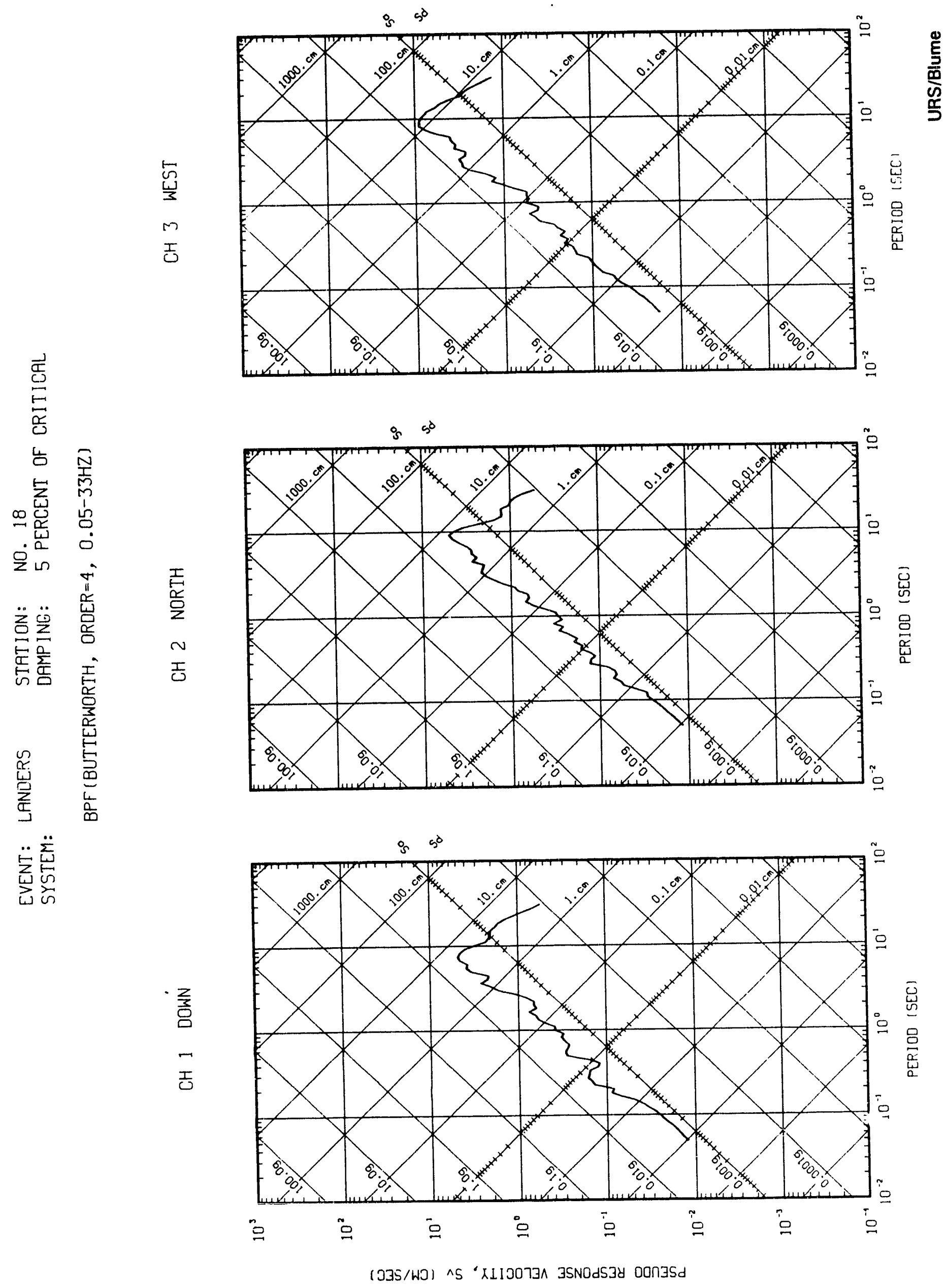


()

-

O

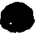

0

0 


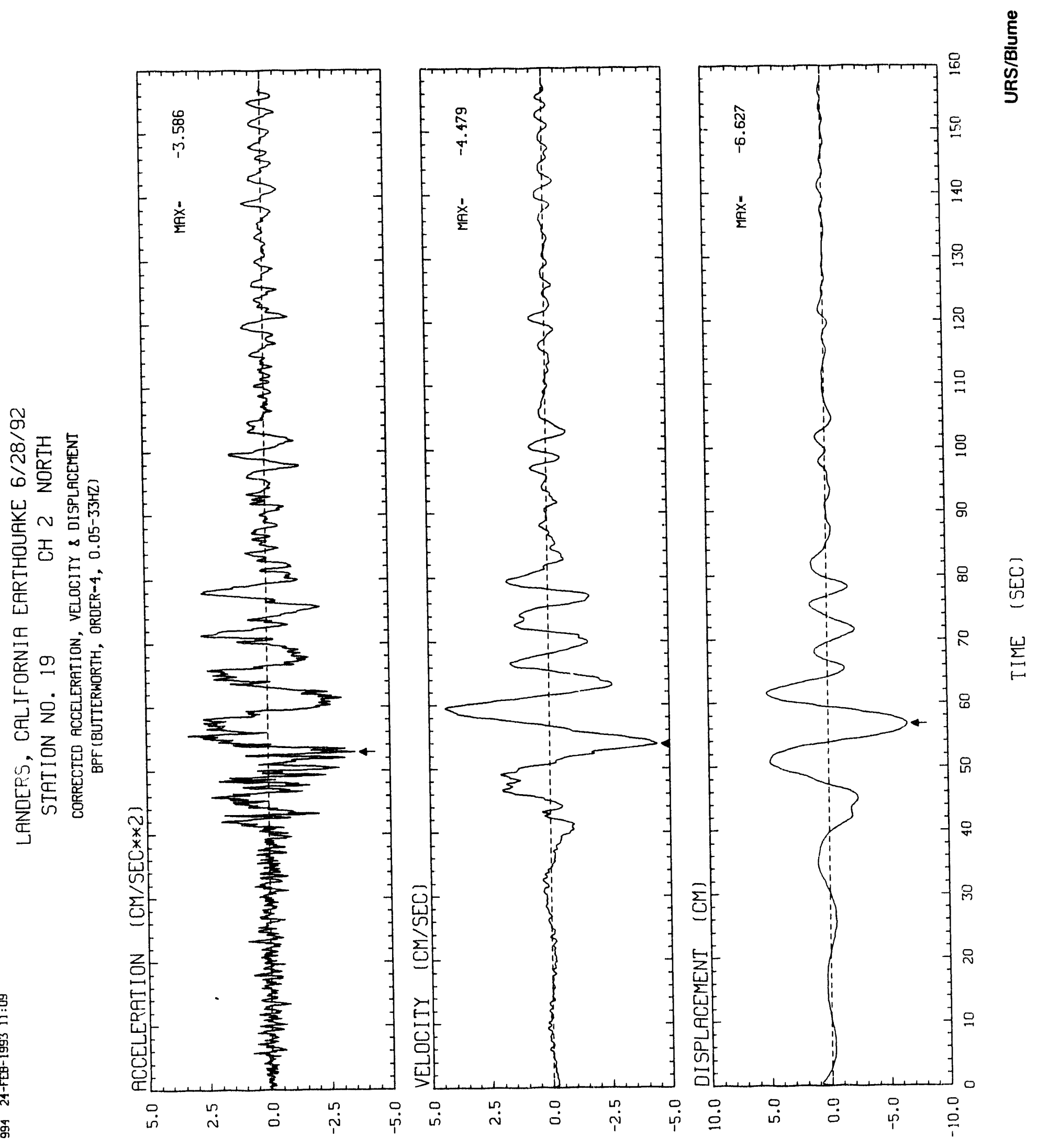

JONI I 7dWH 


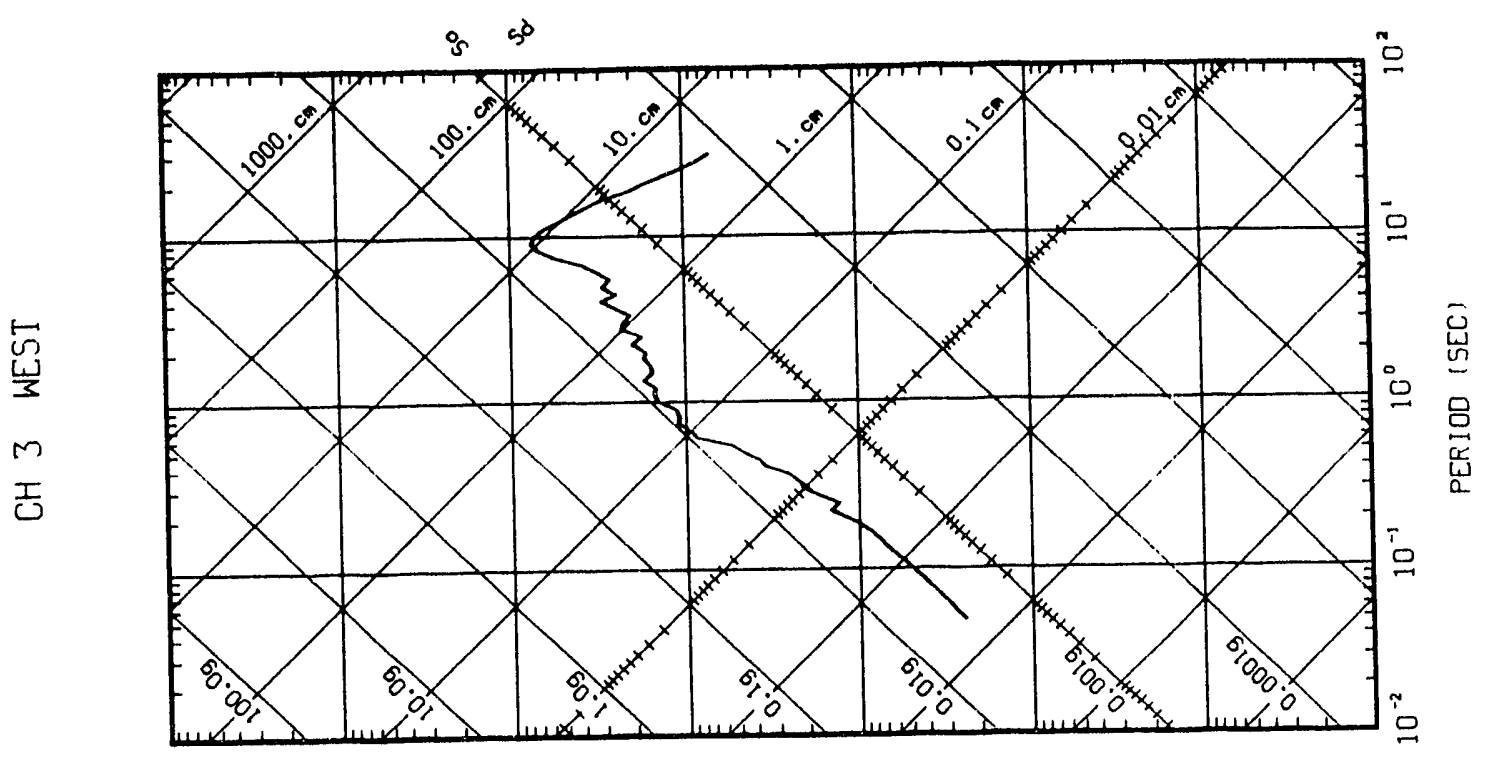

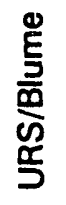
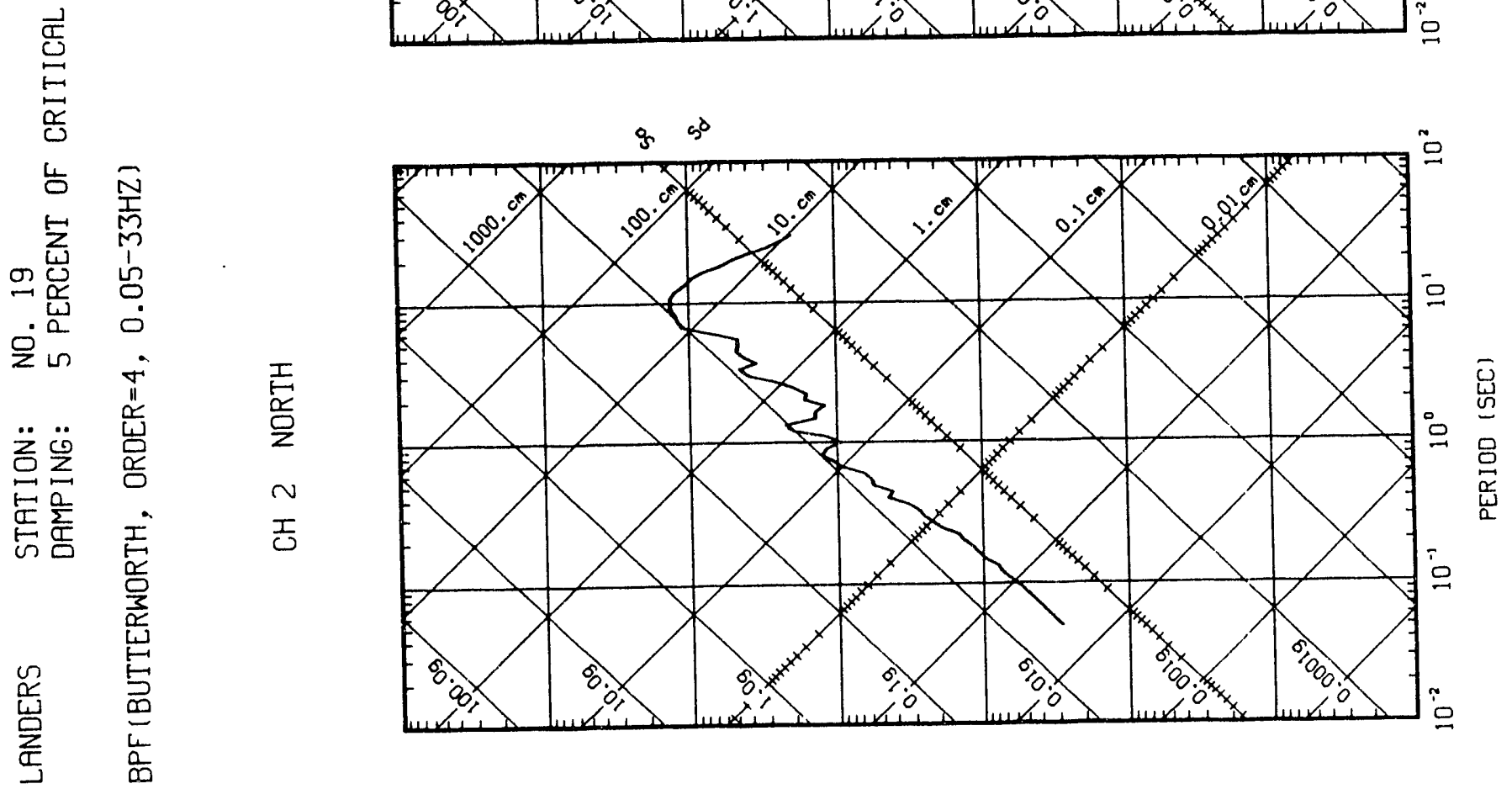

苍岕

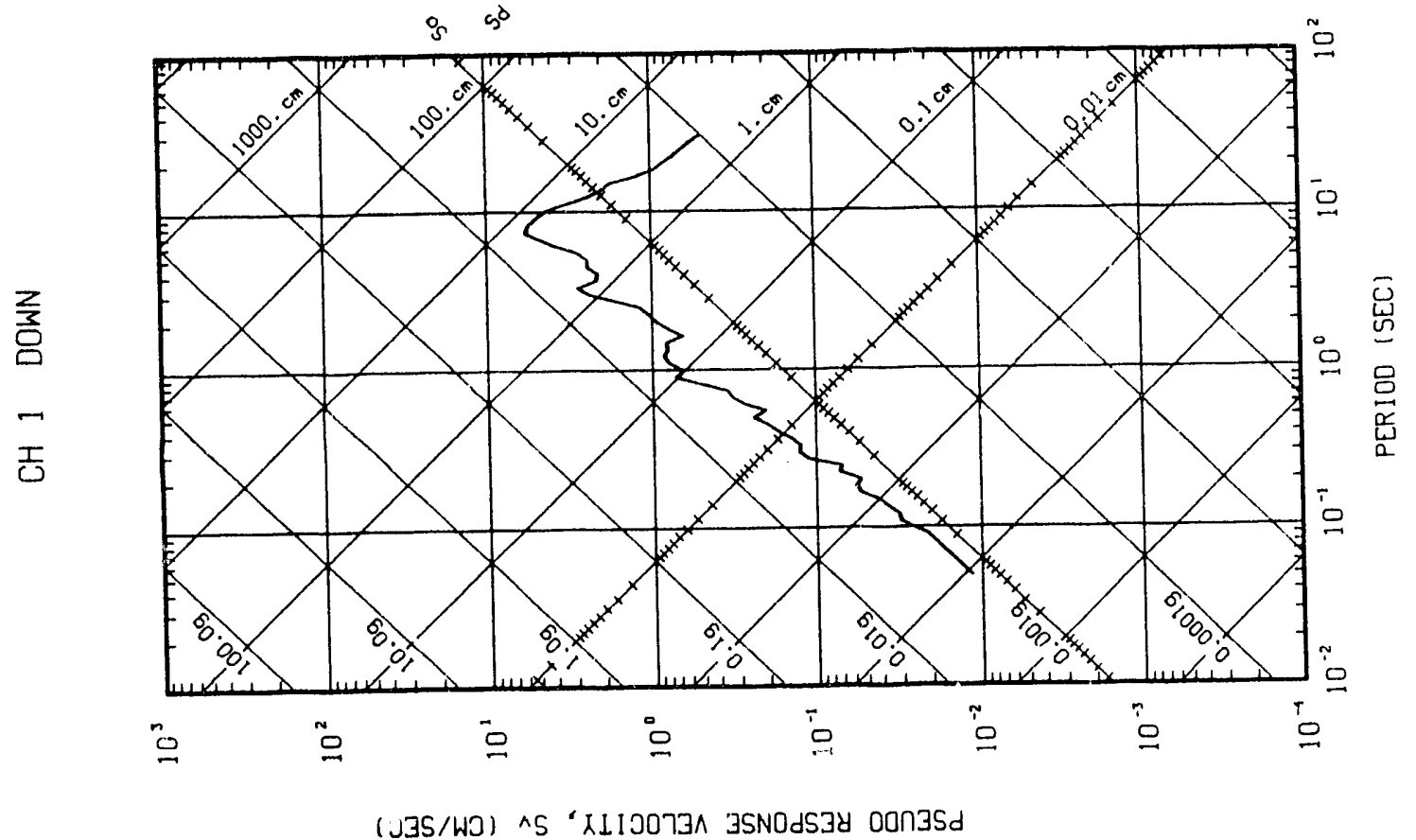


DISTRIBUTION LIST: JAB-10733-TM9

U.S. Department of Energy

Nevada Operations Office

P.O. Box 98518

Las Vegas, NV 89193-8518

J.E. O'Donnell

Technical Information Resource Center

U.S. Department of Energy

Office of Science Education and

Technical Information

P.O. Box 62

Oak Ridge, TN 37831

(2 Copies) 

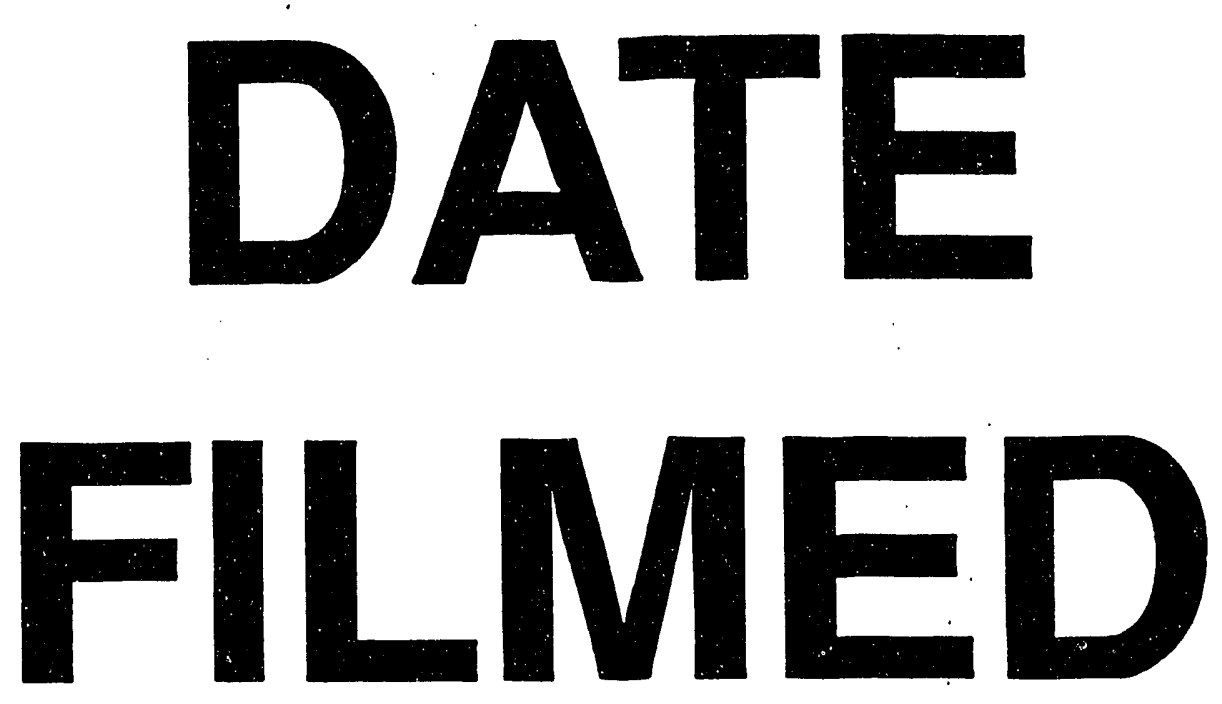

$9 / 20 / 93$
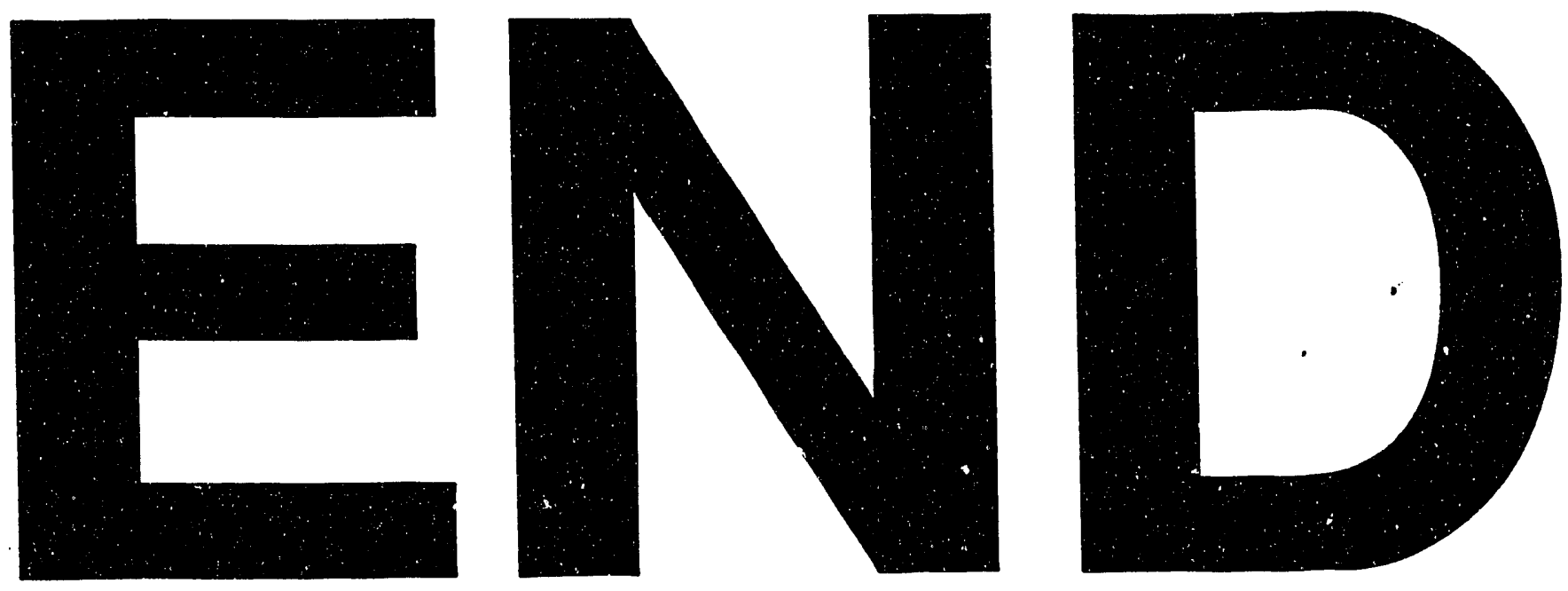
